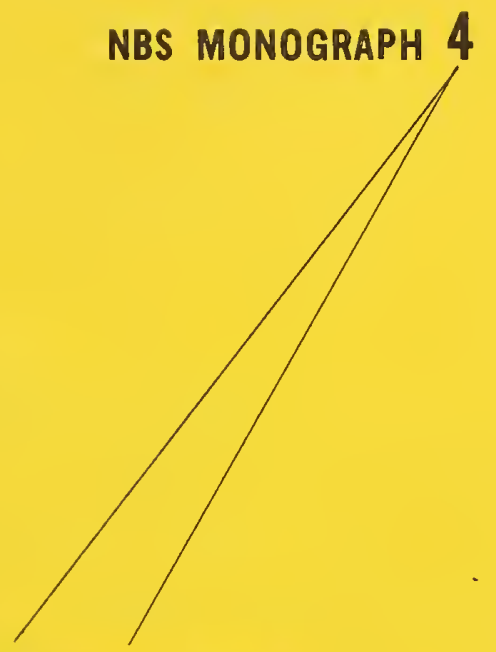

CRPL Exponential Reference Atmosphere

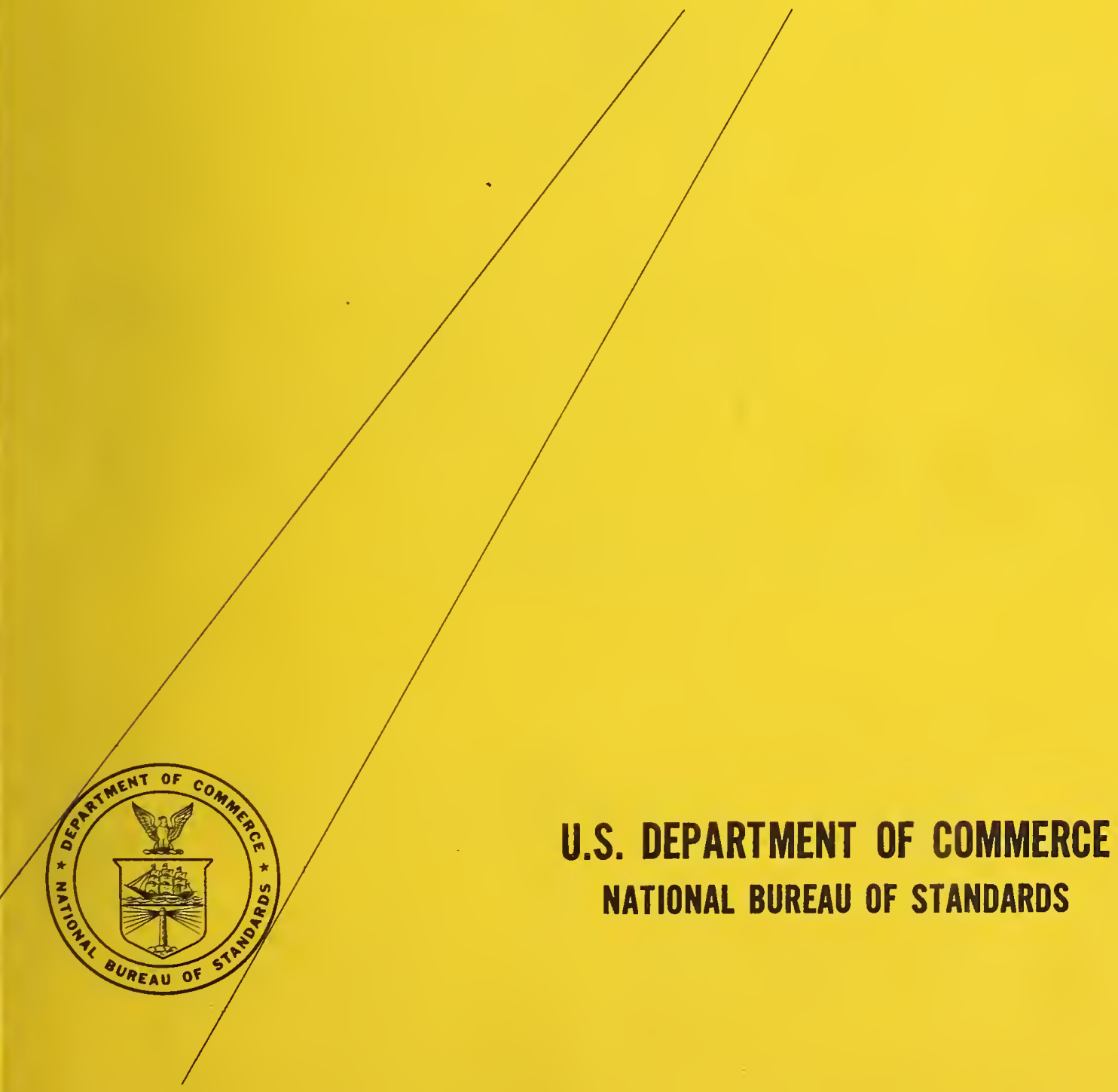




\section{THE NATIONAL BUREAU OF STANDARDS}

\section{Functions and Activities}

The functions of the National Bureau of Standards are set forth in the Act of Congress, March 3, 1901, as amended by Congress in Public Law 619, 1950. These include the development and maintenance of the national standards of measurement and the provision of means and methods for making measurements consistent with these standards; the determination of physical constants and properties of materials; the development of methods and instruments for testing materials, devices, and structures; advisory services to government agencies on scientific and technical problems; invention and development of devices to serve special needs of the Government; and the development of standard practices, codes, and specifications. The work includes basic and applied research, development, engineering, instrumentation, testing, evaluation, calibration services, and various consultation and information services. Research projects are also performed for other government agencies when the work relates to and supplements the basic program of the Bureau or when the Bureau's unique competence is required. The scope of activities is suggested by the listing of divisions and sections on the inside of the back cover.

\section{Publications}

The results of the Bureau's work take the form of either actual equipment and devices or published papers. These papers appear either in the Bureau's own series of publications or in the journals of professional and scientific societies. The Bureau itself publishes three periodicals available from the Government Printing Office: The Journal of Research, published in four separate sections, presents complete scientific and technical papers; the Technical News Bulletin presents summary and preliminary reports on work in progress; and Basic Radio Propagation Predictions provides data for determining the best frequencies to use for radio communications throughout the world. There are also five series of nonperiodical publications: Monographs, Applied Mathematics Series, Handbooks, Miscellaneous Publications, and Technical Notes.

Information on the Bureau's publications can be found in NBS Circular 460, Publications of the National Bureau of Standards $(\$ 1.25)$ and its Supplement (\$1.50), available from the Superintendent of Documents, Government Printing Office, Washington 25, D.C. 
UNITED STATES DEPARTMENT OF COMMERCE · Frederick H. Mueller, Secretary NATIONAL BUREAU OF STANDARDS - A. V. Astin, Director

\section{CRPL Exponential Reference Atmosphere}

B. R. Bean and G. D. Thayer

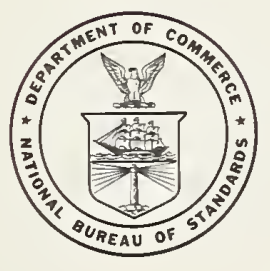

National Bureau of Standards Monograph 4

Issued October 29, 1959 


\section{Contents}

1. Introduction _...

2. Background $\ldots$

3. Basic theory

4. Numerical ray tracing _._.

5. Accuracy of the tables

6. Tables of the CRPL exponential reference atmospheres

7. References_...... 9

8. Appendix A. Tables of profile variables for the CRPL exponential reference atmosphere -...-

9. Appendix B. Tables of refraction variables for the exponential reference atmos-

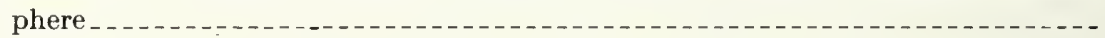




\title{
CRPL Exponential Reference Atmosphere
}

\author{
B. R. Bean and G. D. Thayer
}

\begin{abstract}
The background and development of an exponential model of atmospheric radio refractivity, the CRPL Exponential Reference Atmosphere, is outlined. A set of ray tracings for the model is presented in the form of tables of refraction variables for the complete range of observed values of surface refractive index. A detailed analysis of the accuracy of the ray tracing and tabulation methods is made for these tables. The variables are presented as numbers between 1 and 10 multiplied by the appropriate power of 10 , thus maintaining a maximum number of significant figures. The tables may be used for the solution of practical refraction problems involving elevation angle errors, range errors, and similar quantities.
\end{abstract}

\section{Introduction}

Some exponential models of atmospheric radio refractivity have been introduced recently to replace the standard $4 / 3$ earth treatment for applications involving long-range transmission paths, forward scatter predictions, or radar tracking at high altitudes $[1,2,3,4,5] .^{1}$ One of these models, developed at the Central Radio Propagation Laboratory and called the "CRPL Exponential Reference Atmosphere," has been adopted for use by the National Bureau of Standards in predictions of refraction phenomena.

In this monograph, the background of the exponential reference atmosphere will be outlined and a set of radio ray tracings presented. These ray tracings may be used for the solution of many problems involving refraction by means of the exponential reference atmosphere.

\section{Background}

In dealing with refraction problems it is convenient to use the radio refractivity defined by:

$$
N \equiv(n-1) \times 10^{6},
$$

rather than the radio refractive index $n$. The basic predictor of refraction effects used in the exponential reference atmosphere is the surface value of refractivity at the transmitting point, $N_{3}$. The reasons for this become clear upon examination of the normal, or average, $N$ structure of the atmosphere. It is found that the gradient of $N$ which, with the usual assumption of horizontal homogeneity, is always with respect to height, is, on the average, a function of the surface value, $N_{s}$. This is illustrated in figure 1 where values of $\Delta N$, the difference between $N_{s}$ and the value of $N$ at $1 \mathrm{~km}$. above the surface, are plotted against $N_{s}$. The data represent 888 six-yr monthly means from 45 U.S. weather stations representing many climatically and geographically diverse locations. As shown, $\Delta N$ is approximately an exponential function of $N_{s}$, the relationship being expressed by:

$$
-\overline{\Delta N}=7.32\left[\exp \left\{0.005577 \bar{N}_{s}\right\}\right],
$$

which is a least squares fit of $\ln (-\overline{\Delta N})$ to $\bar{N}_{s}$.

1 Figures in brackets indicate the literature references at the end of this monograph.
It has also been found that the average decay of $N$ with height is very nearly exponential. This can be seen from inspection of figure 2 where averages and extremes of $N$ structure are shown for medium high elevations on a semilogarithmic plot.

The exponential reference atmosphere was defined simply as that family of $N$ profiles having a simple exponential decay with height and passing through the values $N_{s}$ at the surface and $N_{s}+\Delta N$ at a height of $1 \mathrm{~km}$ above the surface where $\Delta N$ is found from eq (1). $N(h)$ is then given by:

$$
N=N_{s} \exp \left\{-c_{e}\left(h-h_{s}\right)\right\},
$$

where $h_{s}$ is the surface elevation, $h$ is the altitude above mean sea level, and $c_{e}$ is the decay constant given by:

$$
c_{e}=\ln \frac{N_{s}}{N_{s}-\Delta N} .
$$

Table 1 shows values of $N_{s}, \Delta N, c_{e}$, and $h_{s}$ for those profiles which were used in making up the tables of refraction variables given in appendix B (a more complete table of this sort may be found in appendix A). The values of $h_{s}$ 


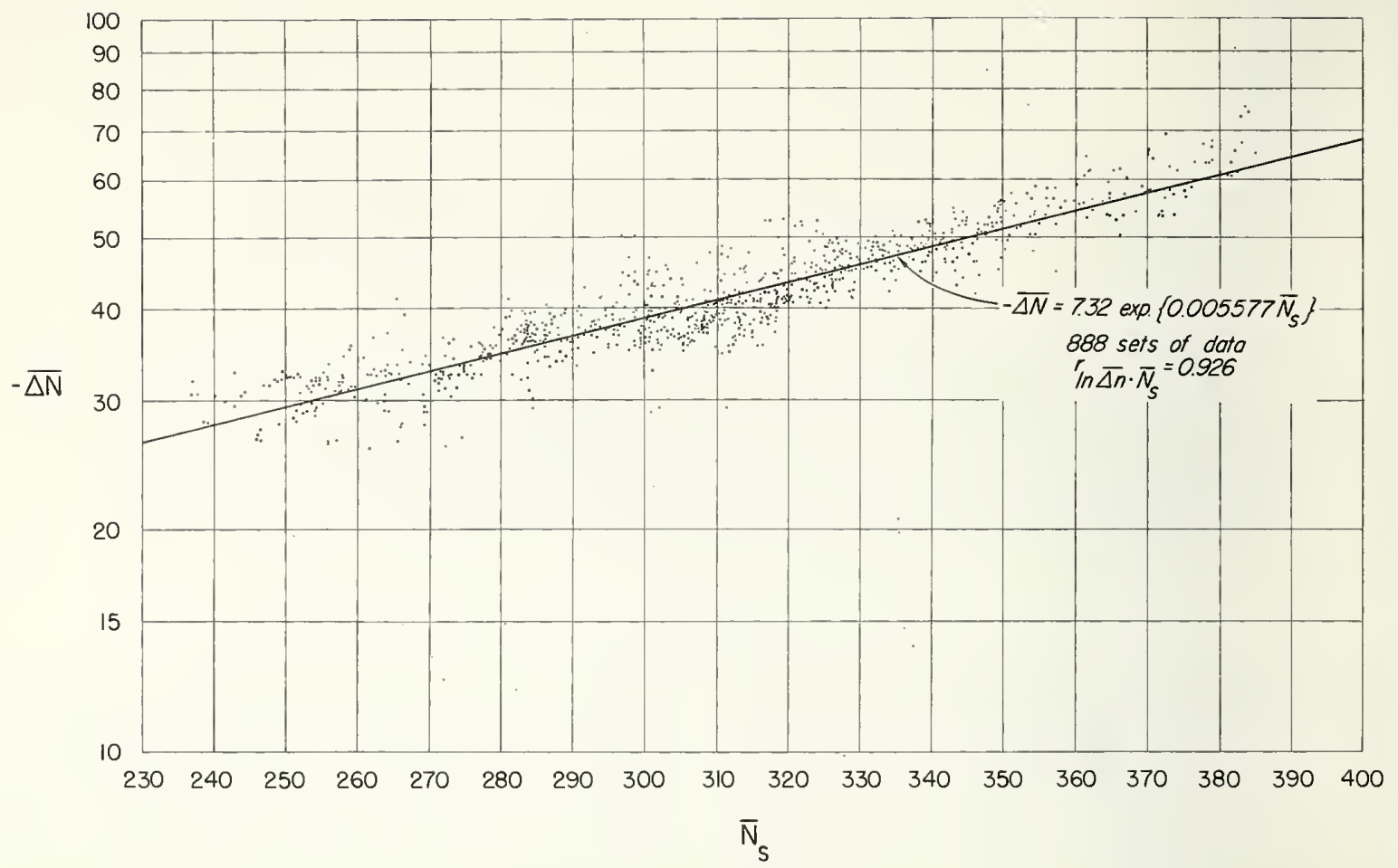

FigURE 1. Six-year mean $\Delta \mathrm{N}$ as a function of 8-year mean $\mathrm{N}_{8}$ at 0300 and 1500 G.m.t. for 45 U.S. weather stations.

were chosen to reflect the average elevation dependence of $N_{s}$.

It is found that several important advantages accrue from this particular choice of model, among which are:

(1) The $N$ profiles involved prove to be a fairly good representation of average $N$-structure over

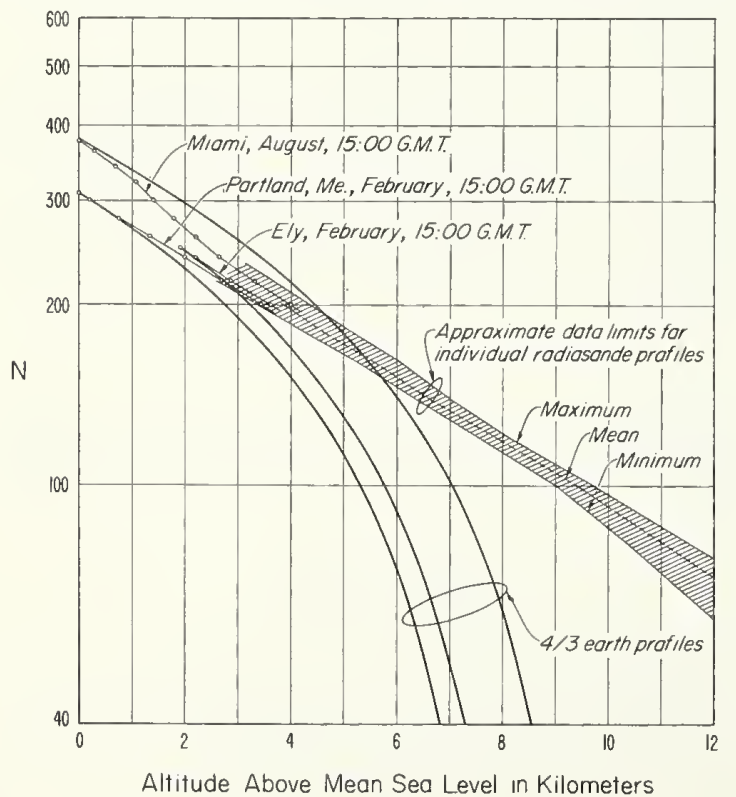

FIgURE 2. Means and extremes of radio-refractivity height structure, and a comparison with extreme $4 / 3$ earth profiles. the first $3 \mathrm{~km}$ above the surface, a critical range for refraction effects at low angles to the horizon.

(2) The resulting relationship between the $N$-gradient near the surface and $N_{s}$ is found to be very close to the observed average relationship (for 5-yr mean profiles), being within \pm 3 percent over the normally encountered range of $N_{s}$ (from 300 to 365 for sea level, temperate zone stations). This agreement is a requirement for the successful prediction of average refraction effects at elevation angles close to the horizontal.

(3) The resulting profiles and their associated gradients are continuous functions of height and are in reasonably good agreement with the actual

TABLE 1. Exponential reference atmosphere profiles contained in appendix $B$

\begin{tabular}{|c|c|c|c|c|}
\hline$N_{s}$ & $h_{\delta}$ & $\Delta N$ & $c_{0}$ & $\begin{array}{l}\text { Effective } \\
\text { earth's radius } \\
\text { factor, }{ }^{*} k\end{array}$ \\
\hline $\begin{array}{l}N \\
200.0 \\
252.9294 \\
313.0 \\
344.5244 \\
377.2161\end{array}$ & $\begin{array}{r}f t \\
10,000 \\
5,000 \\
700 \\
0 \\
0\end{array}$ & $\begin{array}{c}N \\
-22.3318 \\
-30.6000 \\
-41.9388 \\
-50.0000 \\
-60.0000\end{array}$ & $\begin{array}{c}k m-1 \\
0.118400 \\
.126255 \\
.143859 \\
.156805 \\
.173233\end{array}$ & $\begin{array}{l}\text { 1. } 17779 \\
\text { 1. } 25551 \\
1.40229 \\
1.52479 \\
1.71320\end{array}$ \\
\hline $\begin{array}{l}404.8565 \\
450.0 \\
289.0\end{array}$ & $\begin{array}{l}0 \\
0 \\
0\end{array}$ & $\begin{array}{l}-70.0004 \\
-90.0406 \\
-36.684830\end{array}$ & $\begin{array}{c}.189829 \\
.223256 \\
0.13574771\end{array}$ & $\begin{array}{l}\text { 1. } 95921 \\
\text { 2. } 77761 \\
1.3332410\end{array}$ \\
\hline
\end{tabular}

${ }^{*} k \equiv \frac{1}{1+\frac{r_{0}}{n_{s}}\left(\frac{d n}{d h}\right)_{h_{s}}} \quad$ (note that $\left.\frac{d n}{d h}<0\right) . \quad r_{0}=a+h_{s}$ where $a$ is taken as $6373.0150 \mathrm{~km}(3,960 \mathrm{miles})$. 
observed refractivity at all heights at frequencies sufficiently high so that the refractivity is not influenced by the ionization in the ionosphere.

An illustration of the effectiveness of the exponential reference atmosphere in predicting refraction effects is given by figure 3 , where the values of angular ray-bending are plotted against height for a ray starting at zero elevation angle. The agreement between the average bending for a large number of profiles and the average of the values predicted by the exponential reference atmosphere is seen to be quite good. Note however that the bending versus height predicted by the $4 / 3$ earth model is markedly in disagreement with the observed for most heights.

The preceding background material is intended only as an outline of the development of the exponential reference atmosphere. The remainder of this monograph will be devoted to an analysis of ray tracing theory and its application to the expo- nential reference atmosphere with particular emphasis on the preparation of the tables contained in appendix $\mathbf{B}$.

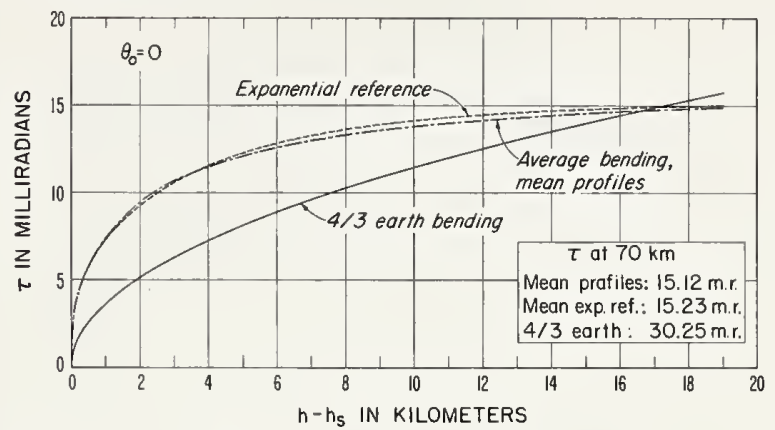

Figure 3. Average radio-ray bending for 225 -yr. mean radio-refractivity profiles as a function of height, compared with the average of the values predicted by the exponential reference atmosphere for each profile and the values predicted by the $4 / 3$ earth treatment.

\section{Basic Theory}

The geometry of the radio-ray-refraction problem is illustrated in figure 4 , and the variables used in the following development are defined in the same figure. The path of the radio ray is the curved line $R$, which will also be used to represent the length of the ray path from the source at $r_{0}$ to the point at $r$. If the transmitter is a radar set, the observed elevation angle to the target at $r$ would be $\theta_{0}$, the true elevation angle would be $\beta$; thus the elevation error angle, $\epsilon$, is given by:

$$
\epsilon \equiv \theta_{0}-\beta \text {. }
$$

The angle $\tau$ is a basic bieasure of refraction effects, and will be called the bending since it represents the angle through which the ray has been bent from its original direction due to refraction. It can be seen from inspection of figure 4 that:

$$
\theta-\theta_{0}=\phi-\tau
$$

from purely geometric considerations.

If it is assumed that the refractive index is spherically stratified with respect to the earth, then Snell's law (for the ray path) may be expressed as:

or

$$
n r \cos \theta=n_{0} r_{0} \cos \theta_{0}=\text { constant, }
$$

\section{$(1$}$$
\left.1+N \times 10^{-6}\right)(a+h) \cos \theta
$$

$$
=\left(1+N_{s} \times 10^{-6}\right)\left(a+h_{s}\right) \cos \theta_{0},
$$

where the radial distance from the center of the earth, $r$, equals $a+h$. The zero or $s$ subsci ipts in (7) refer to the initial values at the earth's surface. Equation (7) may be used to calculate the local elevation angle, $\theta$, at any point along the ray and thus affords a complete description of the ray path. If:

and

$$
n_{2} r_{2} \cos \theta_{2}=n_{1} r_{1} \cos \theta_{1},
$$

and

$$
\begin{aligned}
& n_{2}=n_{1}+\Delta n, \\
& r_{2}=r_{1}+\Delta r,
\end{aligned}
$$

$$
\theta_{2}=\theta_{1}+\Delta \theta
$$

where $\Delta n, \Delta r$, and $\Delta \theta$ are infinitesimals, then

$$
n_{1} r_{1} \cos \theta_{1}=\left(n_{1}+\Delta n\right)\left(r_{1}+\Delta r\right) \cos \left(\theta_{1}+\Delta \theta\right),
$$

and in the limits as $\Delta r$ approaches zero this becomes:

$$
n_{1} r_{1} \cos \theta_{1}=\left(n_{1}+d n\right)\left(r_{1}+d r\right)\left(\cos \theta_{1}-d \theta \sin \theta_{1}\right) .
$$

If (8) is expanded and products of differentials are omitted, then one obtains the differential equation:

$$
\begin{aligned}
\tan \theta d \theta & =\frac{d n}{n}+\frac{d r}{r} \\
d \theta & =\frac{d n}{n} \cot \theta+\frac{d r}{r} \cot \theta .
\end{aligned}
$$

Noting that $d r \cot \theta / r=r d \phi / r=d \phi$, and, from geometry, $d \theta=d \phi-d \tau$, one obtains the classic expression for the bending of a radio ray $[6,7]$ :

$$
d \tau=-\frac{d n}{n} \cot \theta
$$

or

$$
\tau_{[1,2\}}=-\int_{n 1}^{n 2} \frac{d n}{n} \cot \theta
$$




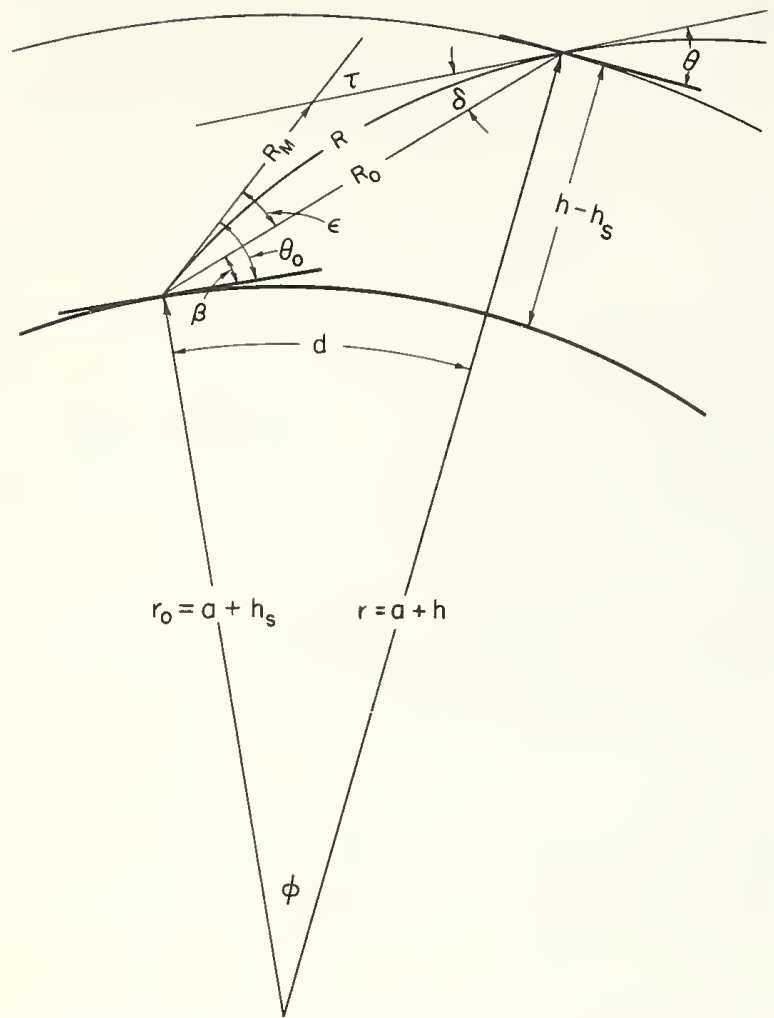

FIGURE 4. The geometry of the radio-ray refraction problem.

Note that (9) defines downward bending as a positive quantity.

If the value of $\tau$ has been obtained for some particular point $(r, n)$ then the angle $\epsilon$ may be found by the following scheme:

$$
n r \cos \theta=n_{0} r_{0} \cos \theta_{0}
$$

from (6), and:

$$
r \cos (\theta+\tau-\epsilon)=r_{0} \cos \left(\theta_{0}-\epsilon\right) .
$$

(This is the equation of a straight line along the slant range, $R_{0}$.) Combining these two equations, employing sum and difference trigonometric formulae, and solving for $\epsilon$ will yield:

$\epsilon=\operatorname{Arctan}\left\{\frac{\cos \tau-\sin \tau \tan \theta-n / n_{s}}{\frac{n}{n_{s}} \tan \theta_{0}-\sin \tau-\cos \tau \tan \theta}\right\}$.

A similar expression for the angle $\delta$ may be derived:

$\delta=\operatorname{Arctan}\left\{\frac{\frac{n_{s}}{n}-\cos \tau-\sin \tau \tan \theta_{0}}{\sin \tau-\cos \tau \tan \theta_{0}+\frac{n_{s}}{n} \tan \theta}\right\}$.
The distance along the earth's surface from $r_{0}$ to $r$ is found by:

$$
d=r_{0} \phi=r_{0}\left(\theta-\theta_{0}+\tau\right)
$$

The true distance from $r_{0}$ to $r$, called the slant range, $R_{0}$, is found from the law of sines or cosines:

$$
R_{0}=\frac{r \sin \phi}{\cos \beta} \sqrt{r_{0}^{2}+r^{2}-2 r_{1} r_{2} \cos \phi} .
$$

The range along the ray path, called the geometric range, $R$, is found by integrating over the differential path lengths:

$$
R=\int_{0}^{R} d R=\int_{r_{0}}^{r} \frac{d r}{\sin \theta}
$$

while the effective or electrical (actually radio) range, $R_{e}$, is found by:

$$
R_{e}=\int_{0}^{R} n d R=\int_{r_{0}}^{r} \frac{n d r}{\sin \theta} .
$$

The radio range error, $\Delta R_{e}$, and the geometric path length difference, $\Delta R$, are defined as:

$$
\Delta R_{e} \equiv R_{e}-R_{0}
$$

and:

$$
\Delta R \equiv R-R_{0}
$$

It should be remembered that the above equations are subject to the two usual restrictions of ray tracing:

(1) The refractive index does not change appreciably in a wavelength.

(2) The fractional change in the spacing between neighboring rays (initially parallel) must be small in a wavelength.

Condition (1) will not be violated by the exponential reference atmospheres since there are no discontinuities in $d n / d r^{2}$ Condition (2) should be satisfied if:

$$
\frac{\Delta N}{N_{s}}<0.002 f_{k c}
$$

which would restrict the ray tracings for the exponential reference atmosphere to frequencies over about $110 \mathrm{kc}$ (for $N_{s}=450$, for $N_{s}=200$ the lower limit would be about $55 \mathrm{kc}$ ).

2 This assumes also that $\left|\frac{d n}{d r}\right|<\frac{1}{r_{0}}$, and $\theta_{0} \geq 0$. 


\section{Numerical Ray Tracing}

Because of the great amount of calculation necessary to obtain a set of ray tracings for the reference atmosphere it was advantageous to resort to digital computing machinery. A complex ray-tracing program had been written for the IBM 650, utilizing the floating point mode (8 digits plus exponent), which was designed to handle arbitrary refractive index profiles having up to 100 layers and it was decided to analyze the exponential reference atmosphere with this program. This section will be devoted to an analysis of the ray tracing techniques employed in the machine program.

The utilization of digital computer techniques for this type of ray tracing necessitates certain changes and alterations in the equations of refraction outlined in the previous section.

To begin with, the basic accuracy of the ray tracing depends upon the accuracy with which the local elevation angles can be calculated using Snell's law. In order to determine an angle in the neighborhood of 1 milliradian $(\mathrm{mr})$ to an accuracy of 8 significant figures from the cosine of that angle, it is necessary to know the value of the cosine to 14 figures $(\cos x=0.999999 \times x x x x x x x$, i.e.: the 6 nines are nonsignificant) or to 1 part in $10^{-14}$.

Rather than employ the relatively slow doubleprecision techniques required for the machine evaluation of eq (6) an alternative formulation of Snell's law was obtained by writing:

$$
1-\cos \theta=1-\frac{n_{0} r_{0}}{n r} \cos \theta_{0} \text {, }
$$

and taking advantage of the fact that $2 \sin ^{2} \theta / 2=1-$ $\cos \theta$, the following equation was derived:

$\theta=2 \operatorname{Arcsin}\left\{\frac{r_{0}}{2 r}\left[2 \sin ^{2} \frac{\theta_{0}}{2}+\frac{r-r_{0}}{r_{0}}-\right.\right.$

$$
\left.\left.\frac{\left(N_{s}-N\right)}{n} \times 10^{-6} \cos \theta_{0}\right]\right\}^{1 / 2} \text {. }
$$

This equation is exact, and if each of the variables employed is correct to 8 figures, then $\theta$ will have an error no greater than 5 parts in the eighth figure. Since in performing ray tracings at initial elevation angles from 0 to $900 \mathrm{mr}$ the value of theta will never be as large as 1 radian, the maximum error in calculating $\theta$ by this method should be $5 \times 10^{-8}$ radians, or in general, not more than about $10^{-5}$ percent of the elevation angle.

With an accurate method of calculating the local elevation angles at various points along the ray path, the primary remaining problem is to compute accurately the total bending of the ray to each selected point. For this purpose a profile of $N$ versus height is entered into the machine. The local elevation angle for each of the profile points may be calculated by means of eq (18), and if it is assumed that

$$
\frac{d N}{d h}=\text { constant }
$$

for each layer defined by succeeding pairs of points of the $N$ profile, it can be shown that:

$$
\Delta \tau_{[1,2]}=\frac{\left(N_{1}-N_{2}\right)}{\bar{n}_{[1,2]}} \times 10^{-6} \cot \bar{\theta}_{[1,2]}
$$

where:

$$
\bar{\theta}_{[1,2]}=\frac{\theta_{1}+\theta_{2}}{2},
$$

and the only approximation is made by letting:

$$
\int_{1}^{2} \frac{d n}{n} \cot \theta=\frac{2}{n_{1}+n_{2}} \int_{1}^{2} d n \cot \theta=\frac{1}{\bar{n}_{[1,2]}} \int_{1}^{2} d n \cot \theta .
$$

Since $n_{1}-n_{2}$ never exceeds about $80 \times 10^{-6}$ between layer heights in the profiles, the maximum error entailed by this approximation cannot be very large (it is actually less than $10^{-4} \%$ of $\Delta \tau$ in the worst case).

It is evident from this method that $\tau$ is essentially the weighted summation of the profile $N$ gradients, where the $\cot \bar{\theta}_{i}$ are the weighting factors. It is important to note that, since for small $\theta \cot \theta \doteq \frac{1}{\theta}$, the weighting factors are very large for the first few layers if $\theta_{0} \doteq 0$. It is thus expected that refraction effects at very-low elevation angles would be extremely sensitive to the initial gradient of $N$.

The integral for $\tau$ was thus computed as the sum of $\Delta \tau$ for each layer:

$$
\tau=\sum_{i=1}^{m} \Delta \tau_{i}
$$

where the only error of any magnitude is that made by assuming $d n / d h=$ constant for each layer. In order to minimize this error it is necessary to restrict the thicknesses of each layer of the profile such that: $\Delta \tau_{1} \leq$ constant, where the constant is some arbitrary angular amount. For the profiles presented in this paper the layer thicknesses were chosen such that $\Delta \tau$ was always less than $2 \mathrm{mr}$ for the first increment, and less than $3 \mathrm{mr}$ for all succeeding increments.

The approximate size of $\Delta \tau_{i}$ was found from the relation $\Delta \tau_{i} \doteq \Delta N_{i} / \theta_{A}$ where $\theta_{A}$ is the approximate value of $\bar{\theta}_{i}$. These were calculated for $\theta_{0}=0$, in which case $\theta_{\mathrm{A}} \doteq \sqrt{\bar{h}} / \sqrt{k r_{0} / 2}$ where $k$ is the effective earth's radius factor for the surface gradient and $\bar{h}$ is the mean height of the layer for which $\Delta \tau_{i}$ is to be calculated.

Thus:

$$
\underset{\sqrt{h_{i}}}{\Delta N_{i}} \sqrt{k r_{0} / 2}<\text { constant. }
$$


It is thus possible to represent the given profile adequately with about 25 or less layers of constant $N$-gradient. It is also possible to calculate bending using the approximation: $n=$ constant for each layer. However it has been found that in order to insure sufficient accuracy about 1,000 layers must be used to approximate the profile with this method [8].

The calculation of the distance is straight forward:

$$
d_{i}=r_{0}\left(\theta_{i}-\theta_{0}+\tau_{i}\right)
$$

where $i$ refers to the value of each variable at the top of the $i$ th layer.
Calculations of the elevation error angle are for convenience made to depend on calculations of the angle $\delta$ :

$$
\epsilon+\tau-\delta,
$$

since $\delta$ is never larger than $1 / 2 \tau$ and is thus more easily calculated than $\epsilon$. (Over the first few increments of height the ray path is approximately a circular arc, and thus $\epsilon \doteq \delta \doteq 1 / 2 \tau$, however as the range increases without limit the elevation angles formed by the ray path and the slant range "path" both approach $\pi / 2$ and thus $\delta \rightarrow 0$. This, of course, implies that $\lim _{R_{0} \rightarrow \infty} \epsilon=\lim _{h \rightarrow \infty} \epsilon=\tau$ since $\tau=\epsilon+\delta$.) Equation (11) for $\delta$ is modified to:

$$
\delta=\operatorname{Arctan}\left\{\frac{\frac{\left(N_{s}-N\right)}{n} \times 10^{-6}+[1-\cos \tau]-\sin \tau \tan \theta_{0}}{\sin \tau-\cos \tau \tan \theta_{0}+\frac{n_{s}}{n} \tan \theta}\right\}
$$

for accuracy in calculation.

The slant range is calculated with the law of cosines formulation (13), reduced to:

$$
R_{0}=\sqrt{\left(r-r_{0}\right)^{2}+2[1-\cos \phi]}
$$

again for accuracy of calculation.

The geometric path length along the ray is calculated by the summation of the incremental path length through each layer (which will be denoted as $\Delta s$ to avoid confusion with the range error, $\Delta R)$ :

$$
R=\sum_{i=1}^{m} \Delta s_{i}
$$

which in turn is calculated by a law of cosines formulation similar to eq (25):

$$
\Delta s_{i}=\sqrt{\left(r_{i}-r_{i-1}\right)^{2}+2\left[1-\cos \left(\phi_{i}-\phi_{i-1}\right)\right]} .
$$

Since the ray path through each layer is very nearly a circular arc, the maximum error in computing $\Delta s_{i}$ from (27) (assuming $\Delta \tau$ is exact) is:

$$
\text { error } \begin{aligned}
\left(\Delta s_{i}\right) & \doteq\left[1-\frac{2 \sin \left(\frac{\Delta \tau_{i}}{2}\right)}{\Delta \tau_{i}}\right] \times 100 \% \\
& \doteq-\frac{\Delta \tau_{i}^{2}}{24} \times 100 \% \text { (to } 7 \text { sig. fig.) }
\end{aligned}
$$

and since, as stated earlier, $\Delta \tau_{i}$ is never as large as 0.003 radians, error $\left(\Delta_{s i}\right)<0.0000375 \%$, and hence: error $(R)<0.00039 \% \doteq 2.5 \mathrm{~m}$ maximum since for the "worst case" i.e.: $N_{s}=450$, (the errors discussed in this and following sections are generally a maximum for the profile with the largest $N$ gradient, in the case of the exponential reference atmosphere, the $N_{s}=450$ profile), $\theta_{0}=0$, total $\tau=31.5 \mathrm{mr}$, there would be about 10 increments of $3 \mathrm{mr}$ each (if calculated with the minimum number of layers), and $R_{0} \doteq 650 \mathrm{~km}$ when $\tau=31.4 \mathrm{mr}$, essentially the total amount of bending. Actually there are some 30 layers calculated when $\tau=31.4 \mathrm{mr}$, and the calculated maximum error in $R$ is only 0.0000514 percent of $650 \mathrm{~km}$ or about $0.334 \mathrm{~ms}$. It is felt that calculation of ranges to an accuracy of $\pm 0.5 \mathrm{~ms}$ or less is sufficient for any valid use to which the exponential reference atmosphere might be put. It should also be borne in mind that the accuracy to which the distance along earth's surface, the slant range, the geonietric path length, and the radio range, can be calculated is also dependent on the accuracy to which the angle $\phi$ has been calculated; this point will be discussed further in the section dealing with the accuracy of the tables.

The radio range, (or "electrical path length", "effective path length", etc.), is calculated simply? as: ${ }^{2}$

$$
R_{e}=\sum_{i=1}^{m} \Delta s_{i}^{\prime}
$$

where: ${ }^{2}$

$$
\Delta s_{i}^{\prime} \equiv \int_{i-1}^{i} n d R=\bar{n}_{i} \Delta s_{i} \doteq \frac{\left(n_{i}+n_{i-1}\right)}{2} \Delta s_{i} .
$$

Assuming that $\Delta s_{i}$ has been calculated accurately, the error in eq $(29)$ for $\Delta s^{\prime}{ }_{i}$ depends on the extent to which:

$$
\frac{1}{R_{i}-R_{i-1}} \int_{i-1}^{1} n d R=\frac{n_{i}+n_{i-1}}{2} .
$$

Since $n_{i}-n_{i-1}$ never exceeds $80 \times 10^{-6}$,and the error (defined as the difference between the above terms) will never be as large as 2 percent of $n_{i} \sim n_{i-1}$, the maximum error in eq (29) should be 
less than $1.6 \times 10^{-4}$ percent. The worst case (again $N_{s}=450, \theta_{0}=0$ ) yields a calculated maximum error of $2.8 \times 10^{-5}$ percent (at $R_{e} \doteq 800 \mathrm{~km}$ ) or about $0.224 \mathrm{~m}$. Note that the weighted \&verage of $n$ is always smaller than the arithmetic mean of the end-point values, $n_{i}$ and $n_{i-1}$; this indicates that the errors due to this approximation will be of opposite sign to those due to using eq (26) and (27) to calculate $R$. Since these two errors will thus oppose each other when calculating $R_{e}$, this quantity will tend to be more accurate than $R$, the calculated error for the $N_{s}=450, \theta_{0}=0$ case being only $-0.110 \mathrm{~m}$ (11 cm error in about $800 \mathrm{~km}$, or $1.37 \times 10^{-5}$ percent).

The errors discussed in the above paragraphs are, of course, much larger percentage-wise when applied to the values of the range errors:

$$
\begin{gathered}
\Delta R=R-R_{0} \\
\Delta R_{e}=R_{e}-R_{0}
\end{gathered}
$$

in the case previously mentioned as representing the largest errors $\left(N_{s}=450, \theta_{0}=0, R_{0} \geq 800 \mathrm{~km}\right)$ the percentages are: Error $(\Delta R) \doteq-0.67$ percent maximum; i error $\left(\Delta R_{e}\right) \doteq-0.06$ percent maximum)

\section{Accuracy of the Tables}

In the preceding section on numerical raytracing the errors inherent in the equations and approximations employed were discussed at some length. It is important now to deal with those errors peculiar to the particular mode of computation used in developing the tables, i.e.: those errors which could be avoided by employing a more accurate method of calculation using the same equations and approximations. The following material is a rather detailed analysis of errors; for a concise presentation of the maximum errors likely to occur for each variable the reader is referred to the table at the beginning of appendix B.

The basic source of error of this type is derived from the accuracy in the calculations of the $N$ values used in the refractivity profiles. The profiles for the exponential reference atmosphere were calculated to an accuracy of $\pm 5 \times 10^{-11}$ in $n$ (or $\pm 5 \times 10^{-5}$ in $N$ units). Thus the quantity $N_{s}-N_{0 \cdot 01}$, the difference between surface $N$ and $N$ at $10 \mathrm{~m}$ above the surface can be calculated to an accuracy of only about 5 significant figures (this is the worst case), resulting in the following maximum errors: Error $(\theta)< \pm 0.000047 \mathrm{mr} \quad \theta_{0}<4$ $\mathrm{mr}$; error $(\theta)< \pm 0.000005 \mathrm{mr} \theta_{0}>4 \mathrm{mr}$; and error in $\Delta \tau<0.0001 \mathrm{mr}$ (from this cause only). These are random rounding errors and will not accumulate over a large number of height increments. For $\theta_{0}$ larger than $10 \mathrm{mr}$ the accuracy will be unaffected by the mentioned errors in $N$, with the result that the largest error in $\theta$ (for $\theta_{0}$ of 400 and $900 \mathrm{mr}$ ) would be 3 or 4 units in the eighth place: Error $(\theta)<0.000004 \mathrm{mr} 10$ $\mathrm{mr}<\theta_{o}<100 \mathrm{mr}$; error $(\theta)<0.00004 \mathrm{mr} \quad \theta_{0}>100$ $\mathrm{mr}$. Hence the maximum error expected to be encountered in $\theta$ at any point is less than $5 \times 10^{-8}$ radians.

The accuracy with which $\tau$ can be calculated depends primarily on the accuracy with which $N_{i}-N_{i-1}$ is known, so that $\tau$ can be relied upon to 5 or 6 figures, depending on whether or not it is over $10 \mathrm{mr}$. Except for the first increment at $\theta_{0}=0$, an error in the calculated value of $\Delta \tau$ for some particular height increment due to a rounding error in $N_{i}-N_{i-1}$ will in general be at least $2 / 3$ offset by an error of opposite sign over the following height increment. An error in the first increment at $\theta_{0}=0$ will not usually be made up later due to the excessively large weighting factor given the error by $\cot \bar{\theta}$ in that case. Upon investigating the average error in $\tau$ at any height caused by the above mentioned errors, it is found that: partial error $(\tau)<0.0003 \mathrm{mr}$.

This is an additional error in $\tau$ due to the difference between the true exponential decay of $N$ and the linear approximation of $N$ versus height assumed by the ray-tracing procedure. Errors of this type will be a maximum for the first increment of $\tau$ for $\theta_{0}=0$, because of the rapid change of $\cot \theta$ near $\theta=0$. An equation has been derived for the difference in the incremental bending for the first layer at $\theta_{0}=0$ between the linear and exponential profiles (having the same value of $N_{s}$ and $N$ at $\left.r-r_{0}=\Delta h_{1}\right)$, which is the error in $\Delta \tau_{1}$ due to the linear approximation:

$$
\text { error }\left(\Delta \tau_{1}\right)=\left(\Delta \tau_{1}\right)\left[\left(1-\frac{N_{s}-N_{1}}{c_{e} N_{s} \Delta h_{1}}\right)-\Delta h_{1}\left(\frac{c_{e}}{3}-\frac{c_{e}^{2} \Delta h_{1}}{10}+\frac{c_{e}^{3}\left(\Delta h_{1}\right)^{2}}{42}-\ldots\right)\right]
$$

error due solely to difference in $N$ gradient assuming same $\cot \theta$

$$
+\sqrt{\left(\frac{1}{r_{0}}-\left[N_{s}-N_{1}\right] \times 10^{-6}\right)^{3}}\left[\frac{N_{s}-N_{1}}{N_{s}}-\frac{2}{5}\right]\left(\Delta h_{1}\right)^{3 / 2} \text { radians }
$$


error due solely to difference in $\cot \theta$ where the subscript 1 refers to values of the variables at the first height increment, $r-r_{0}=\Delta h_{1}$. In the case of the $N_{s}=450$ profile the partial error in $\Delta \tau_{1}$ from the first part of expression (30), amounts to only $0.000760 \mathrm{mr}$, while the remainder of the error in $\Delta \tau_{1}$ from the second part of eq (30) amounts to only $0.00000725 \mathrm{mr}$, or only 1 percent of the first part. These errors drop rapidly with decreasing initial gradient of $N$, so that for the $N_{s}=377.22$ profile the error in $\Delta \tau_{1}$ is only $0.000287 \mathrm{mr}$, or only about $1 / 3$ as much as for the $N_{s}=450$ profile.

Errors in the value of the elevation error angle. $\epsilon$, will be of the same order of magnitude as those in $\tau$ at any point, the method of computation being more precise than the values of $\tau$ used in the calculation.

Errors in the distance, $d$, and the ranges, $R_{0}, R$, $R_{e}$ will be determined by the accuracy to which the angular distance can be calculated,

$$
\phi=\theta-\theta_{0}+\tau,
$$

which in turn depends on the accuracy to which $\theta$ and $\tau$ are known. Since $\theta$ will have a maximum error of about $4 \times 10^{-5} \mathrm{mr}$ and $\tau$ a maximum error of about $8 \times 10^{-4} \mathrm{mr}$, the distance and ranges are calculated at least to an accuracy of about $\pm 5 \mathrm{~m}$. However, if it is assumed that the profile is exact as represented by linear $N$ layers, the accuracy of the distance and ranges increases, with a maximum error of about $\pm 0.6 \mathrm{~m}$. Actually the ranges for the $N_{s}=377.22$ profile are accurate to about \pm 1.8 $\mathrm{m}$, for the $N_{s}=200$ profile the maximum error is of the order $\pm 0.6 \mathrm{~m}$. (about the same as if the profile were actually composed of linear $N$ layers).

The preceding estimates of maximum errors are based on the $\theta_{0}=0$ ray; for any ray at $\theta_{0}>0$ the errors will in general be no larger than the limits imposed by computational accuracy (i.e.: errors not caused by the difference between exponential and linear $N$ layers). Note that for very large $\theta_{0}$ the bending of the ray path is so slight that no significant differences can be detected between the arc lengths along the ray path, i.e.: the geometric range, and the slant range. In these cases the reported $\Delta R$ values are almost entirely one to four parts of the eighth significant figure of $R$, inter- spersed with values given as exactly zero. These are due to random rounding errors, and are often negative. In general, to be considered as significant (or real) a value of $\Delta R$ should be at least as large as two parts in the seventh figure of $R$. An exception to this rule is for $\theta_{0}=900 \mathrm{mr}$; in this case the angle $\phi$ can only be calculated to two significant figures at the low heights, since $\theta-\theta_{0}$ is about $10^{-6}$ radians and $\theta=\theta_{0}$ is about 1 radian, and as a result of internal inconsistencies in the triangles formed by the $\phi_{1}$ and $r_{i}, r_{0}$ combinations, erroneous $\Delta R$ values as large as 0.002 percent of $R_{0}$ are calculated. However, in none of the raytracings is there an entirely erroneous value of $\Delta R$ or $\Delta R_{e}$ reported which is larger than a few centimeters (almost all such values are in the millimeter class, and differences between $R_{0}$ and $R$ as small as $10^{-9} \mathrm{~km}$, or $0.001 \mathrm{~mm}$ are found in some of the ray-tracings for $\theta_{0}=200$ and $400 \mathrm{mr}$ ). A good rule to follow in using the values of $\Delta R$ and $\Delta R_{e}$ given in these ray-tracings would be to consider that any of the values may be subject to an error of at least $\pm 10 \mathrm{~cm}$, and (in the case of the larger values of $\Delta \vec{R}$ ) possibly as much as $\pm 50 \mathrm{~cm}$.

The values shown as "DELTA H" in the tables are the corrections necessary to correct the height given by a simple $4 / 3$ earth atmosphere for the distance given (assumed exact) to the height given (also assumed exact). As a result, the values of DELTA $H$ have an error approximately equal to: error $($ delta $h) \doteq \pm$ (absolute erı or in $d) \frac{h}{d}$ since the distances given are not really exact. The values shown as "DELTA THETA" are the corrections necessary to correct the elevation angle given by a simple $4 / 3$ earth atmosphere at the given distance (assumed exact) to the value calculated for the exponential profile. This quantity is found by:

$$
\Delta \theta=\tau-\frac{\phi}{4}
$$

where $\tau$ and $\phi$ are as calculated for the exponential reference atmosphere at the point where $\Delta \theta$ is desired. The maximum error in this quantity therefore depends mostly on the errors in $\tau$, hence: error $(\Delta \theta)<10^{-6}$ radian.

\section{Tables of the GRPL Exponential Reference Atmospheres}

The tables themselves are simply a presentation of ray tracing variables. In these tables all angles are given in milliradians and all distances in kilometers. For each different profile $\left(N_{s}=\right.$ 200 through 450 ) ray tracings are given for $\theta_{0}$ from 0 to $900 \mathrm{mr}$, in approximately a geometric progression (i.e.: $0,0.5,1,2,4,8,15,30,65,100$, 200,400 , and $900 \mathrm{mr}$ ), at fixed heights along the ray path. From left to right: first is given the SURFACE $N$, or $N_{s}$, to 4 figures, not rounded.
Exact values of $N_{s}$ used are given in table 1 . The next column is the value of $\theta_{0}$, or "INIT(ial) THETA", in milliradians. The "HEIGHT" is given in kilometers. The preceding quantities are given with fixed decimal points, the remaining quantities are listed as floating point numbers in the following fashion: $\pm \mathrm{X} . \mathrm{XXXX} \pm E$ where the first sign refers to the sign of the number, $\mathrm{X} . \mathrm{XXX}$ which is to be multiplied by 10 to the power $\pm E$ depending on the second sign. 
Examples:

$$
\begin{aligned}
0.0000 & =\text { zero } \\
1.2345 & =1.2345\left(\times 10^{\circ}\right) \\
1.23453 & =1.2345 \times 10^{3} \\
-1.23453 & =-1.2345 \times 10^{3} \\
1.2345-3 & =1.2345 \times 10^{-3} \\
-1.2345-3 & =-1.2345 \times 10^{-3} .
\end{aligned}
$$

The following quantities are listed as floating point numbers in this manner: "DELTA H"= correction to $4 / 3$ height at same distance to equal "HEIGHT", in kilometers; "THETA" $=\theta$ or local elevation angle, in milliradians; "DELTA THETA" = correction to $4 / 3$ earth $\theta$ to equal THETA at same distance, in milliradians; "DISTANCE" $=r_{0} \phi$ or distance along earth's surface, in kilometers; "TAU" $=\tau$, angular bending, in milliradians; "ERROR ANGLE" $=\epsilon$, elevation error angle, in milliradians; "SLANT RANGE" $=R_{0}$, straightline distance from ray origin to point at $h, d$, in kilometers; "DELTA $\mathrm{R}^{\prime \prime}=\Delta R$, difference between distance from $h=0$ to given height along ray path and slant range, in kilometers; "DELTA $R-E$ " $=\Delta R_{e}$, difference between radiopath length and slant range, in kilometers.

The following ray-tracing variables have not been printed, but can be easily calculated from those given, as follows:

$$
\phi=\frac{d}{r_{0}}=\frac{\text { "DISTANCE" }}{\text { "INIAL RADIUS" }}
$$

where $r_{0}=6373.0150+h_{s}$ (kilometers)

$$
\begin{gathered}
\phi=\tau-\epsilon=\text { "TAU" - "ERROR ANGLE", } \\
R=R_{o}+\Delta R=\text { "SLANT RANGE" } \\
\text { + "DELTA } R ", \\
R_{e}=R_{o}+\Delta R_{e}=\text { "SLANT RANGE" } \\
\text { +"DELTA } R-\mathrm{E} " .
\end{gathered}
$$

Values of elevation of the ray starting-point, $h_{s}$, are given in table 1 .

The last profile, for $N_{s}=289.0$, is a special profile which was prepared for the purpose of making direct comparisons between $4 / 3$ earth refraction and an exponential profile having the same $N$ gradient at the surface. It is intended for applications where it is desired to replace the $4 / 3$ earth treatment with a single standard atmosphere having the advantage of differing very little from the $4 / 3$ earth near the surface.

The authors wish to acknowledge the technical assistance of Miss B. A. Cahoon, and Mrs. B. J. Weddle, and particularly of K. A. Norton whose help in working out some of the mathematical methods employed was invaluable.

\section{References}

[1] B. R. Bean and G. D. Thayer, On models of the atmospheric radio refractive index,- Proc. IRE 47, No. 5, 740 (May 1959).

[2] B. R. Bean, Atmospheric bending of radio waves, paper presented at International Conference on Electromagnetic Wave Propagation, Liege, Belgium, October, 1958 (to be published by the Academy Press, spring 1959).

[3] L. J. Anderson, Tropospheric bending of radio waves, Trans. Am. Geophys. Union 39, No. 2, 208 (February 1958).

[4] J. R. Bauer, W. C. Mason, F. A. Wilson, Radio refraction in a cool exponential atmosphere, Lincoln
Laboratories, Technical Report No. 186 (August $27,1958)$.

[5] G. Millington, Propagation at great heights in the atmosphere, The Marconi Rev. 21, No. 131, 143, 4th Quarter (1958).

[6] M. Schulkin, Average radio-ray refraction in the lower atmosphere, Proc. IRE 40, 554 (May 1952).

[7] W. M. Smart, Spherical astronomy, Chapter III (Cambridge University Press, London, 1931).

[8] G. H. Millman, Tropospheric effects on radar target measurements, Proceedings of the Seventh Weather Radar Conference, held at the Deauville Hotel, Miami Beach, Florida (November 17-20, 1958). 


\section{Appendix A. Tables of Profile Variables for the GRPL Exponential Reference Atmosphere}

The following equations were used in determining the various profile variables:

$$
\begin{aligned}
& -\Delta N=7.32 \exp \left\{0.005577 N_{s}\right\} \\
& c_{e}=\ln \left\{\frac{N_{s}}{N_{s}+\Delta \bar{N}}\right\} \\
& -d N_{0}=c_{e} N_{s} \\
& k=\frac{1}{1+\frac{r_{0}}{n_{s}}\left(c_{e} N_{s}\right) \times 10^{-6}} n_{s} \equiv 1+N_{s} \times 10^{-6},
\end{aligned}
$$

$r_{0}$ is taken as $6373.014987 \mathrm{~km}$ for all values of $N_{s}$ in these tables.

The table giving $N_{s},-\Delta N, c_{e}$, and $-d N_{0}$ as functions of $k$ was prepared using an iterative method for solution of the transcendental equa-

\begin{tabular}{|c|c|c|c|c|}
\hline$N_{a}$ & $-\Delta N$ & $c$ & $-d N_{0}$ & $k$ \\
\hline 200 & 22.3317700 & 0.118399435 & 23.6798870 & 1. 17769275 \\
\hline 210 & 23.6125966 & .119280212 & 25. 0488444 & 1. 18991401 \\
\hline 220 & 24. 9668845 & .120458179 & 26.5007993 & 1. 20315637 \\
\hline 230 & 26. 3988468 & .121916361 & 28.0407631 & 1. 21752719 \\
\hline 240 & 27.9129385 & 123642065 & 29.6740955 & 1. 23314913 \\
\hline 250 & 29.5138701 & .125626129 & 31.4065323 & 1. 25016295 \\
\hline 260 & 31.2066224 & .127862319 & 33. 2442030 & 1. 26873080 \\
\hline 270 & 32. 9964614 & 130346887 & 35. 1936594 & 1. 28904048 \\
\hline 280 & 34.8889558 & . 133078254 & 37.2619112 & 1.31131073 \\
\hline 290 & 36.8899932 & .136056720 & 39.4564487 & 1. 33579768 \\
\hline 300 & 39.0057990 & .139284287 & 41. 7852861 & 1. $362 \$ 0330$ \\
\hline 310 & 41. 2429556 & 142764507 & 44. 2569972 & 1. 39268608 \\
\hline 320 & 43. 6084233 & .146502381 & 46.8807620 & 1. 42587494 \\
\hline 330 & 46.1095611 & .150504269 & 49.6664087 & 1. 46288731 \\
\hline 340 & 48. 7541501 & .154777865 & 52. 6244741 & 1. 50435338 \\
\hline 350 & 51.5504184 & .159332141 & 55. 7662495 & 1. 55104840 \\
\hline 360 & 54.5070651 & .164177379 & 59. 1038565 & 1. 60393724 \\
\hline 370 & 57. 6332884 & . 169325150 & 62. 6503054 & 1. 66423593 \\
\hline 380 & 60.9388149 & .174783368 & 66.4195799 & 1. 73349938 \\
\hline 390 & 64.4339281 & 180581312 & 70. 4267116 & 1. 81374807 \\
\hline 400 & 68. 1295015 & .186719722 & 74. 6878887 & 1. 90765687 \\
\hline 410 & 72.0370324 & .193220834 & 79. 2205420 & 2. 01884302 \\
\hline 420 & 76. 1686780 & . 200103517 & 84.0434770 & 2. 15232187 \\
\hline 430 & 80.5372922 & .207388355 & 89.1769927 & 2. 31525447 \\
\hline 440 & 85. 1564647 & .215097782 & 94. 6130240 & 2. 51823286 \\
\hline 450 & 90.0405683 & .223256247 & 100.4653113 & 2. 77761532 \\
\hline
\end{tabular}
tions involved. In all these tables the accuracy may be taken as \pm 1 in the last digit listed.

\begin{tabular}{|c|c|c|c|c|}
\hline$-\Delta N$ & $N_{s}$ & $c$. & $-d N_{0}$ & $k$ \\
\hline $\begin{array}{l}20 \\
22 \\
24 \\
26 \\
28\end{array}$ & $\begin{array}{l}180.226277 \\
197.316142 \\
212.917967 \\
227.270255 \\
240.558398\end{array}$ & $\begin{array}{r}0.117626108 \\
.118216356 \\
.119594076 \\
.121491305 \\
.123746115\end{array}$ & $\begin{array}{l}21.1993155 \\
23.3259953 \\
25.4637276 \\
27.6113599 \\
29.7681671\end{array}$ & $\begin{array}{l}\text { 1. } 15617524 \\
1.17457412 \\
1.19366808 \\
1.21348565 \\
1.23406110\end{array}$ \\
\hline $\begin{array}{l}30 \\
32 \\
34 \\
36 \\
38\end{array}$ & $\begin{array}{l}252.929362 \\
264.501627 \\
275.372099 \\
285.621054 \\
295.315731\end{array}$ & $\begin{array}{l}126255291 \\
128950180 \\
131783550 \\
134721962 \\
137741207\end{array}$ & $\begin{array}{l}\text { 31. } 9336703 \\
34.1075325 \\
36.2895127 \\
38.4794288 \\
40.6771452\end{array}$ & $\begin{array}{l}\text { 1. } 25543336 \\
\text { 1. } 27764560 \\
\text { 1. } 30074523 \\
\text { 1. } 32478398 \\
\text { 1. } 34981825\end{array}$ \\
\hline $\begin{array}{l}40 \\
42 \\
44 \\
46 \\
48\end{array}$ & $\begin{array}{l}304.513148 \\
313.261483 \\
321.602888 \\
329.573439 \\
337.204713\end{array}$ & $\begin{array}{r}.140823306 \\
.143955014 \\
.147125889 \\
.150328075 \\
.153555418\end{array}$ & $\begin{array}{l}42.8825481 \\
45.0955611 \\
47.3161107 \\
49.5441405 \\
51.7796106\end{array}$ & $\begin{array}{l}\text { 1. } 37590934 \\
\text { 1. } 40312414 \\
\text { 1. } 43153539 \\
\text { 1. } 46122250 \\
\text { 1. } 49227226\end{array}$ \\
\hline $\begin{array}{l}50 \\
52 \\
54 \\
56 \\
58\end{array}$ & $\begin{array}{l}344.524418 \\
351.557000 \\
358.324138 \\
364.845143 \\
371.137293\end{array}$ & $\begin{array}{r}156803056 \\
160067149 \\
.163344614 \\
.166633002 \\
.169930326\end{array}$ & $\begin{array}{l}54.0224815 \\
56.2727266 \\
58.5303179 \\
60.7952415 \\
63.0674811\end{array}$ & $\begin{array}{l}\text { 1. } 52477960 \\
\text { 1. } 55884863 \\
\text { 1. } 59459364 \\
\text { 1. } 63214058 \\
\text { 1. } 67162830\end{array}$ \\
\hline $\begin{array}{l}60 \\
62 \\
64 \\
66 \\
68\end{array}$ & $\begin{array}{l}\text { 377. } 216108 \\
383.095581 \\
388.788373 \\
394.305974 \\
399.658845\end{array}$ & $\begin{array}{r}173234984 \\
.176545680 \\
.179861358 \\
.183181171 \\
.186504431\end{array}$ & $\begin{array}{l}65.3470266 \\
67.6338699 \\
69.9280046 \\
72.2294300 \\
74.5381454\end{array}$ & $\begin{array}{l}\text { 1. } 71321044 \\
\text { 1. } 75705732 \\
\text { 1. } 80335830 \\
\text { 1. } 85232456 \\
\text { 1. } 90419225\end{array}$ \\
\hline $\begin{array}{l}70 \\
72 \\
74 \\
76 \\
78\end{array}$ & $\begin{array}{l}404.856538 \\
409.907798 \\
414.820650 \\
419.602477 \\
424.260086\end{array}$ & $\begin{array}{r}.189830583 \\
.193159183 \\
.196489873 \\
.199822385 \\
.203156494\end{array}$ & $\begin{array}{l}76.8541525 \\
79.1774555 \\
81.5080567 \\
83.8459677 \\
86.1511914\end{array}$ & $\begin{array}{l}\text { 1. } 95922635 \\
\text { 2. } 01772514 \\
\text { 2. } 08002556 \\
\text { 2. } 14650999 \\
\text { 2. } 21761358\end{array}$ \\
\hline $\begin{array}{l}80 \\
82 \\
84 \\
86 \\
88\end{array}$ & $\begin{array}{l}428.799768 \\
433.227348 \\
437.548229 \\
441.767432 \\
445.889634\end{array}$ & $\begin{array}{r}206492043 \\
.209828917 \\
.213167031 \\
.216506335 \\
.219846812\end{array}$ & $\begin{array}{l}88.5437400 \\
90.9036251 \\
93.2708570 \\
95.6454475 \\
98.0274147\end{array}$ & $\begin{array}{l}\text { 2. } 29383429 \\
\text { 2. } 37574437 \\
\text { 2. } 46400458 \\
\text { 2. } 55938222 \\
\text { 2. } 66277367\end{array}$ \\
\hline $\begin{array}{r}90 \\
92 \\
94 \\
96 \\
98 \\
100\end{array}$ & $\begin{array}{l}449.919193 \\
453.860184 \\
457.716416 \\
461.491458 \\
465.188659 \\
468.811163\end{array}$ & $\begin{array}{r}.223188453 \\
.226531281 \\
.229875327 \\
.233220637 \\
.236567271 \\
.239915290\end{array}$ & $\begin{array}{l}100.4167688 \\
102.8135290 \\
105.2177108 \\
107.6293319 \\
110.0484114 \\
112.4749663\end{array}$ & $\begin{array}{l}\text { 2. } 77523207 \\
\text { 2. } 89800399 \\
\text { 3. } 03257531 \\
\text { 3. } 18073184 \\
\text { 3. } 34463902 \\
\text { 3. } 52694820\end{array}$ \\
\hline
\end{tabular}

TABLE A-1.
TABLE A-2

TABLE A-3

\begin{tabular}{c|c|c|c|c}
\hline$k$ & $N$ & $-\Delta N$ & $c_{\theta}$ & $-d N_{0}$ \\
\hline 1.0 & 0.0 & 0.0 & 0.0 & 0.0 \\
1.2 & 217.689023 & 24.6471681 & .120160519 & 26.1576259 \\
1.3 & 275.037959 & 33.9367000 & .131692114 & 36.2203304 \\
1.4 & 312.297111 & 41.7747176 & .143600133 & 44.8459068 \\
1.5 & 339.003316 & 48.4839018 & .154339490 & 52.3215987 \\
1.6 & 359.298283 & 54.2941700 & .163827653 & 58.8629945 \\
1.7 & 375.341242 & 59.3759008 & .172203063 & 64.6349115 \\
1.8 & 388.391792 & 63.8586055 & .179626805 & 69.7655765 \\
1.9 & 399.243407 & 67.8426334 & .186242834 & 74.3562234 \\
2.0 & 408.424907 & 71.4070090 & .192172034 & 78.4878451 \\
& & & & \\
2.1 & 416.304322 & 74.6148487 & .197514185 & 82.2260091 \\
2.2 & 423.146728 & 77.5171828 & .202351472 & 85.6243634 \\
2.3 & 429.148472 & 80.1557288 & .206751820 & 88.7272277 \\
2.4 & 434.458411 & 82.5649192 & .210771674 & 91.5715264 \\
2.5 & 439.191718 & 84.7734613 & .214458304 & 94.1883108 \\
& & & & \\
2.6 & 443.438906 & 86.8054237 & .217851443 & 96.6038056 \\
2.7 & 447.272272 & 88.6811886 & .220984823 & 98.8403838 \\
2.8 & 450.750273 & 90.4181120 & .223887193 & 100.9172131 \\
$\infty$ & 523.299600 & 135.5109159 & .299693586 & 156.829534 \\
$4 / 3$ & 289.036274 & 36.6922523 & .135758874 & 39.2392391 \\
\hline & & & & \\
\hline
\end{tabular}




\section{Appendix B. Tables of Refraction Variables for the Exponential Reference Atmosphere} tables.

The following table of estimated maximum error's should serve as a guide to the accuracy of the

Errors in elevation angle, $\theta$ :

$$
\left.\begin{array}{cl}
\theta_{0} \leq 4 \mathrm{mr} & \pm 0.00005 \mathrm{mr} \\
4 \mathrm{mr}<\theta_{0}<100 \mathrm{mr} & \pm .000005 \mathrm{mr} \\
\theta_{0} \geq 100 \mathrm{mr} & \pm .00004 \mathrm{mr}
\end{array}\right\} \text { nearly independent of } N_{s}
$$

\begin{tabular}{|c|c|c|c|c|c|c|c|}
\hline $\begin{aligned} N_{s} & = \\
\theta_{0} & =0 \\
\theta_{0} & =1^{\circ} \\
\theta_{0} & =3^{\circ}\end{aligned}$ & $\begin{array}{ll}450 & \\
\pm 0 . & 001 \\
.0003 \\
.00004\end{array}$ & $\begin{aligned} & 404 . 8 \\
& 0 . 00065 \\
& .00015 \\
& .000025\end{aligned}$ & $\begin{aligned} & 377 . 2 \\
& 0.0005 \\
& .0001 \\
& .00002\end{aligned}$ & $\begin{aligned} & 344 . 5 \\
& 0 . 0004 \\
& .00008 \\
& .000017\end{aligned}$ & $\begin{aligned} 313 & \\
0 . & 0003 \\
& .00006 \\
. & 000015\end{aligned}$ & $\begin{aligned} & 252 . 9 \\
& 0 . 0002 \\
& .00005 \\
& .000013\end{aligned}$ & $\begin{aligned} & 200 \\
& 0.0002 \\
& .00005 \\
& .000012\end{aligned}$ \\
\hline
\end{tabular}

Errors in $\tau, \epsilon$ (in milliradians):

Errors in $R_{0}, R, R_{e}$, or $\Delta h$ (in meters):

\begin{tabular}{c|c|c|c|c|c|c|c|}
\hline \hline$N_{s}=$ & 450 & 404.8 & 377.2 & 344.5 & 313 & 252.9 & 200 \\
$\theta_{0}=0$ & \pm 5.0 & 2.7 & 1.8 & 1.2 & 0.8 & 0.65 \\
$\theta_{0} \geq 1^{\circ}$ & 0.4 & 0.3 & 0.25 & 0.2 & .17 & 0.6 \\
\hline
\end{tabular}

Assume that error in $\Delta R$ or $\Delta R_{e}$ is \pm 0.5 percent or $\pm 0.1 \mathrm{~m}$, whichever is larger. 


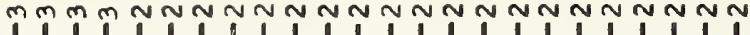

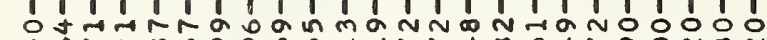
!U

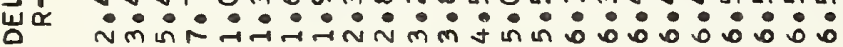

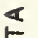

$\propto$

亗

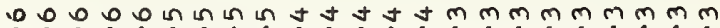

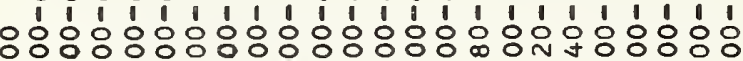

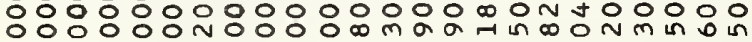
onvm

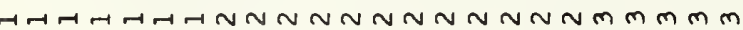

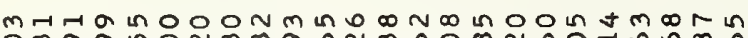

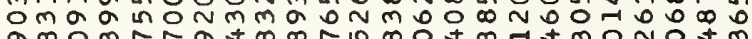
出 यु

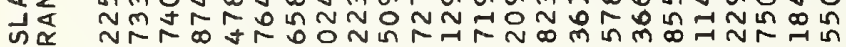

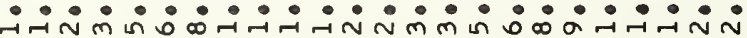

l m

눙

$\stackrel{\alpha}{\alpha} \underset{Z}{\square}$

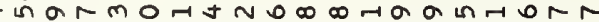

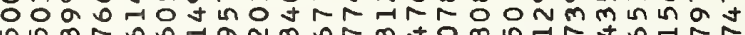

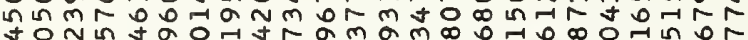
inmj

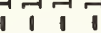

in in 200 t

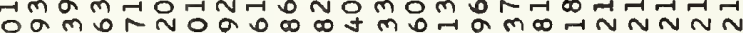

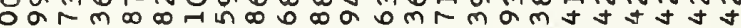
a o

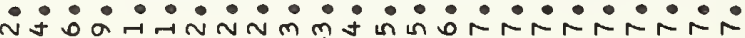

$\underset{5}{\frac{u}{2}}$

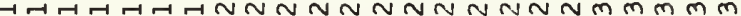

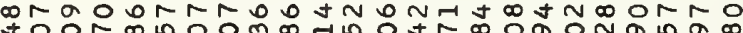

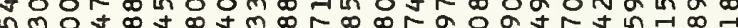

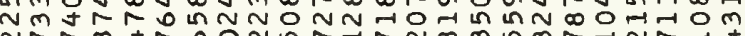
NTN

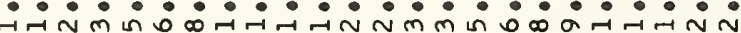

ㄲㅟㄺㅟ

$<4$

㟧

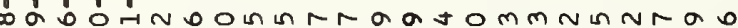
thajorojoormm

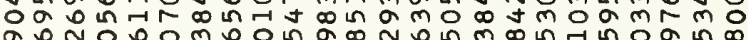

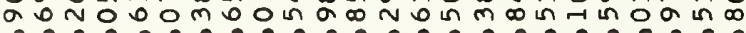

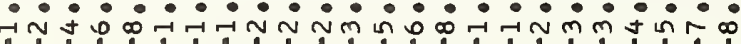
$111,1,1,1,1,1,1,1,1,111,1$

ન

$\underset{⿱ ⺊}{\underline{I}}$

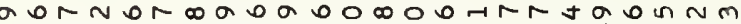
a r O m N N

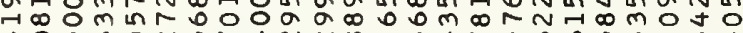
$m 0$ on $0 N$ L

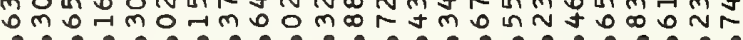

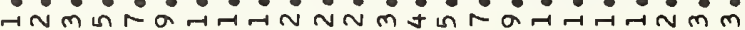

mmmNNNNNFનy mó

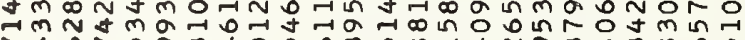

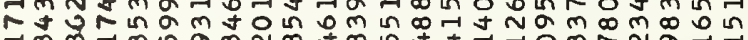

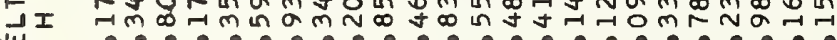

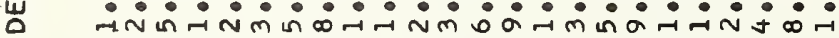

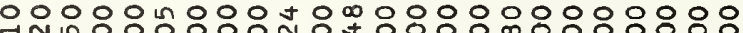

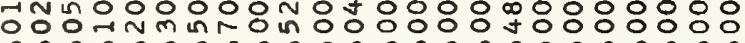

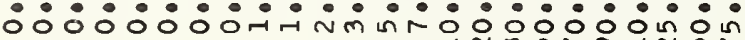
$00000000-1 \mathrm{Nm}$ n

000000000000000000000000

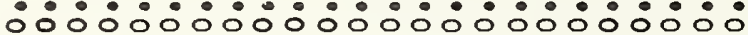

ZI

$\sum_{\substack{u \\ \text { u }}}^{u}$

000000000000000000000000 OO $0 \circ 0000000000000000000$

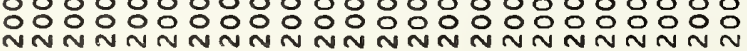

mmmmNNN ง'm aำ

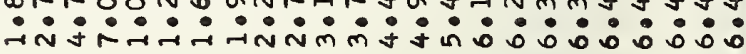

○ o o 0 o

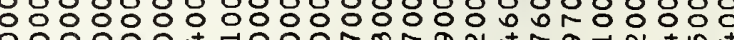

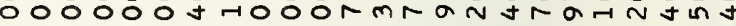

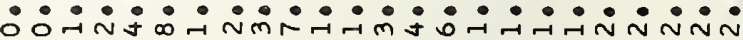

-

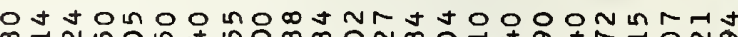
舟 no

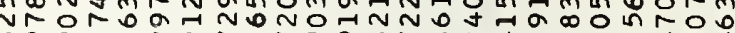

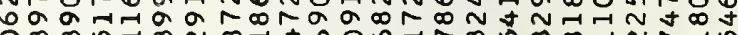

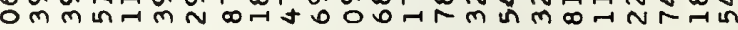

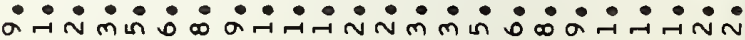

1ै। 1

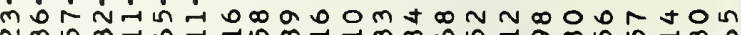

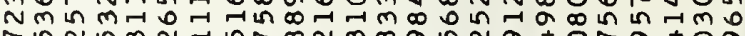

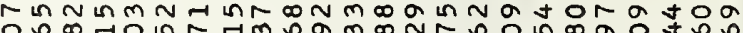
$00 \infty 70$ in

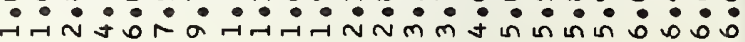

$\overrightarrow{1}-1$

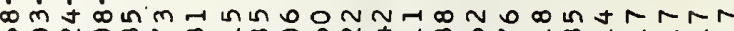
o일 J 0 o toy $\sigma 0 \sigma N N a \infty 00,4 \infty$ in 0 in in in in in in in m

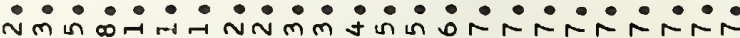

- $-1-H-N N N$ N

$\sim N \$ O \infty$ in กnN

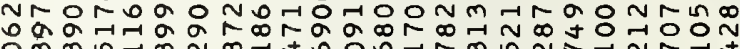
然的开

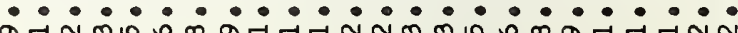

퓌표

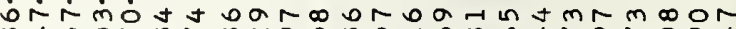
d in or m

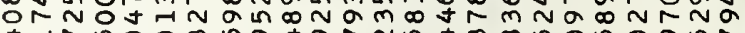
y $\rightarrow$ in $00 \mathrm{~m}$ in $\alpha$ ta $N$ in t $m \infty$ in 0 in $0 \alpha$ in

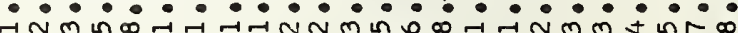

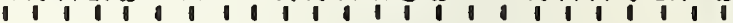

-

ron

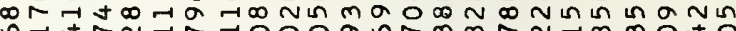
OH TN O

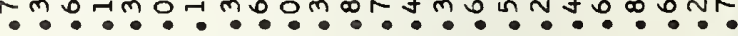

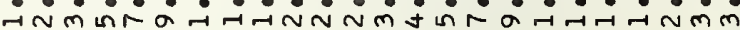

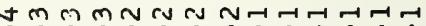

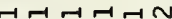

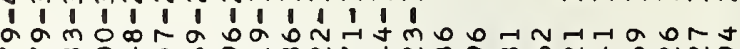

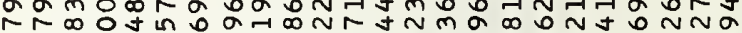
N ง $0-j a n m$ in $n-\pi N m b a r m$ in

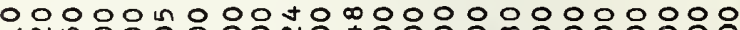

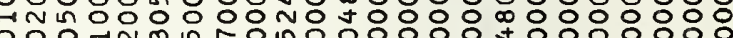
o o onm nn

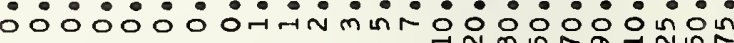
$\rightarrow N m$ s

แn un in in in in in un in in un un un in un in in un un in un un in in

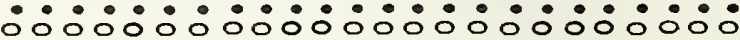

000000000000000000000000

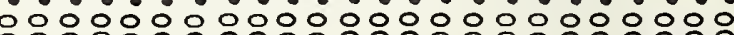
N 
山

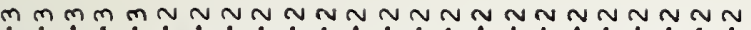

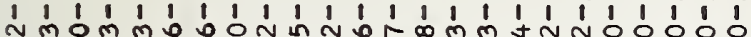

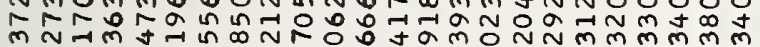

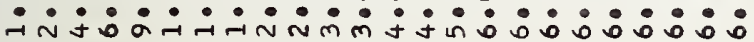

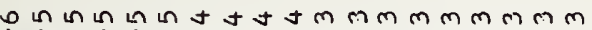

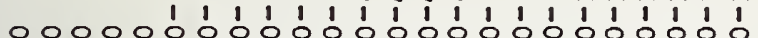

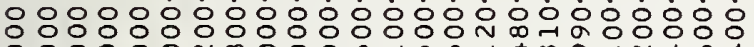

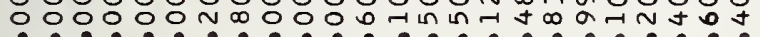

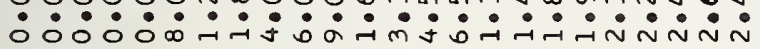

H-H-H-HNNNNNNNNNNNmmmmm

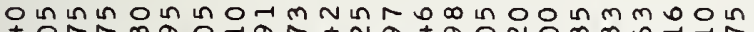
tor $\begin{array}{ll}4 \\ 0\end{array}$

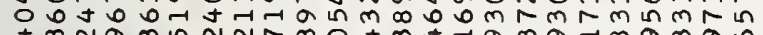

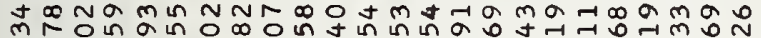

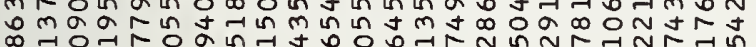

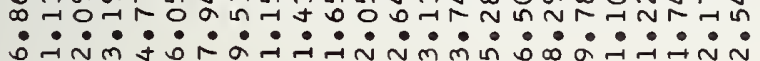

1 $1-1-1-1-1$

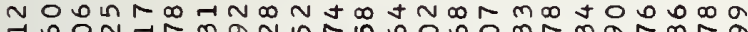
T ONTRMaN N JN

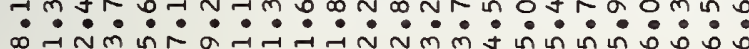

$\overrightarrow{1}-1-1$

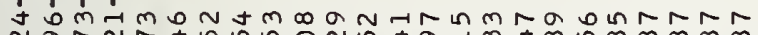

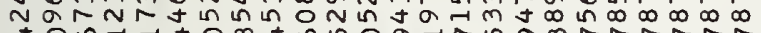
t 0 Drt

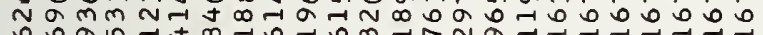

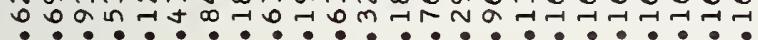

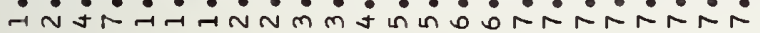

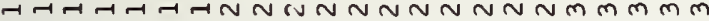

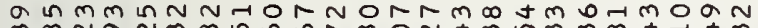

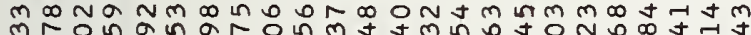

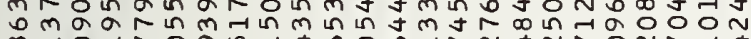
$\infty \rightarrow 0 \rightarrow r 0 \alpha$ เก

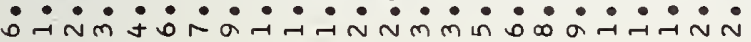

1. -1 -1 $-1-1$

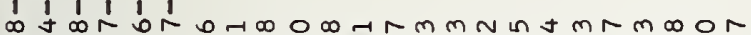
o o a r

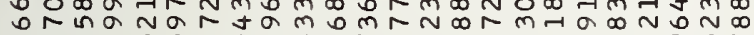

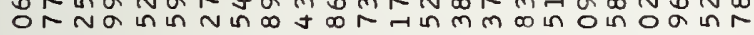

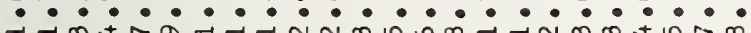
1

-

or D w a O t O in $N \infty$ o ง

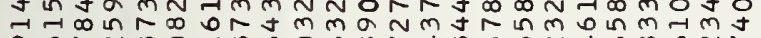
$a$ in

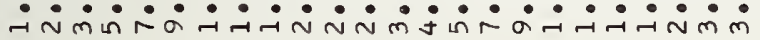

tmmmnNentr-1

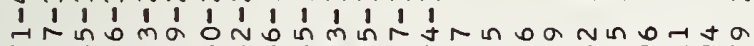

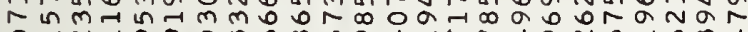
웓ำ -

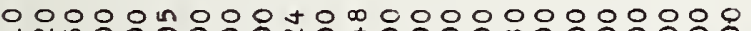

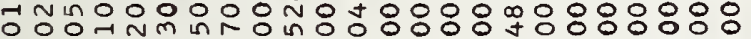

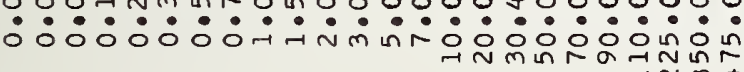

$\ll 000000000000000000000000$

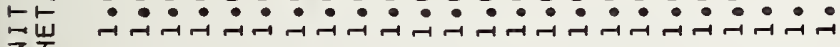

000000000000000000000000

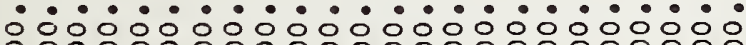
N N

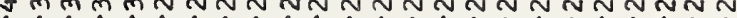

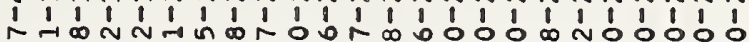
N

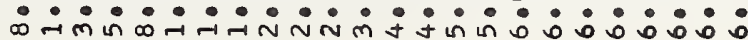

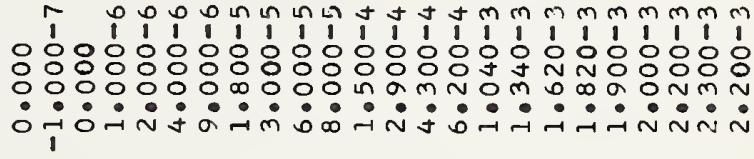
$\neg-\neg-\neg-N \sim N N$ N N N N N

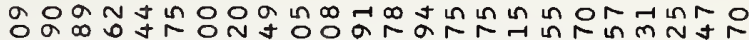

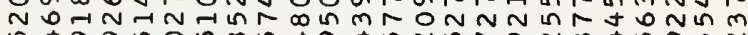

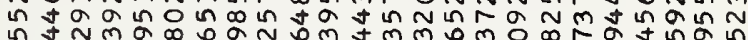

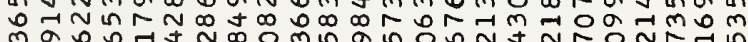

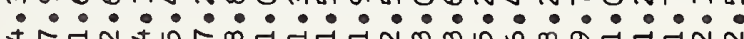

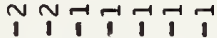

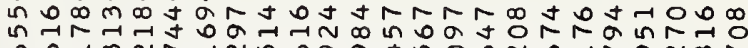

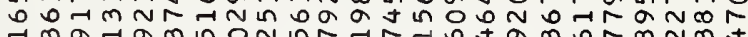
$\because ? \because \because \%$ ?

$\overrightarrow{1} \overrightarrow{1} \overrightarrow{1}$

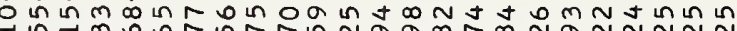
$\rightarrow$ in $\rightarrow$ mor

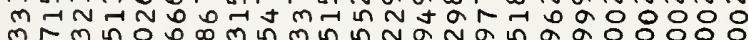

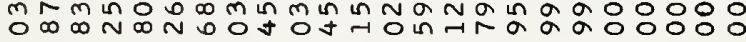
$\therefore \dot{0} \dot{0} \dot{0} \dot{0} \dot{0} \dot{0} \dot{0} \dot{0} \dot{0} \dot{0} \dot{0}$

- $-1-H N N$ N

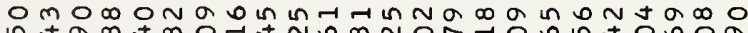

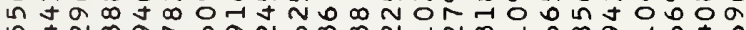

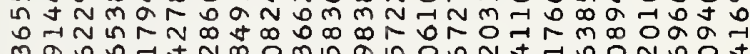

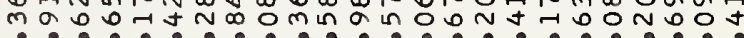

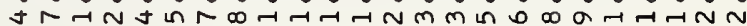

N

0 b 0 j

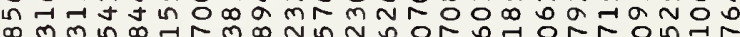

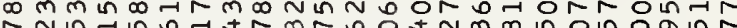

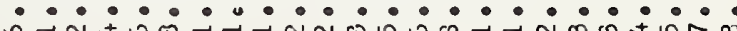

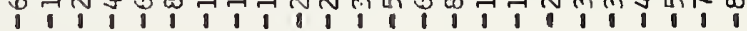

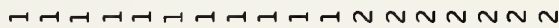

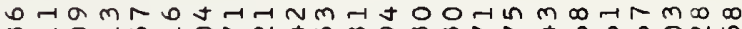

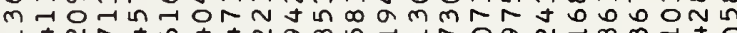

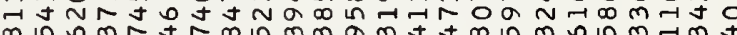

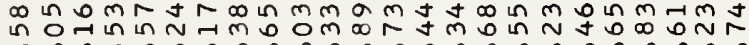

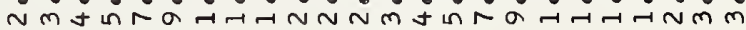

A t

Hศન -

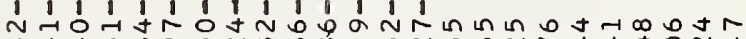

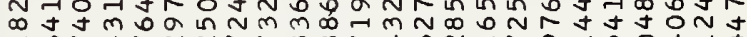

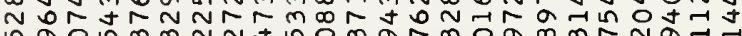
?

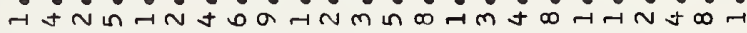

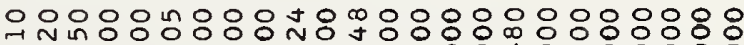

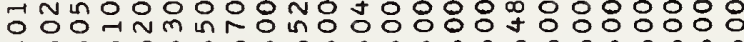

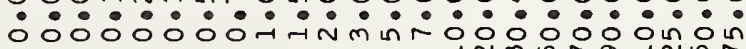

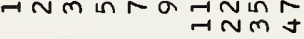

000000000000000000000000

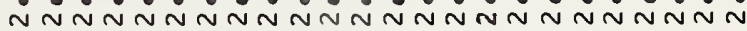

000000000000000000000000

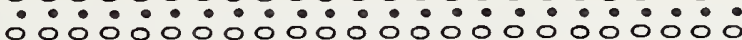
응응응응응응응응응응응응응응응 n N N N N N N N N N N N N N N N N N N N 


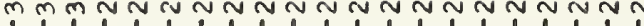

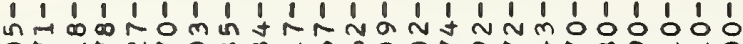

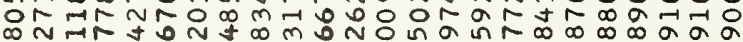
ษ 0
0
1

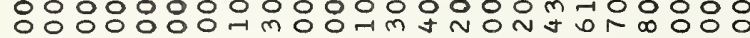

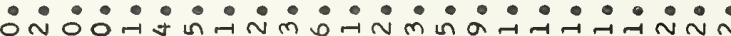
1

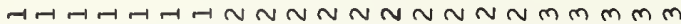

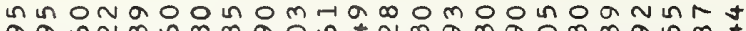

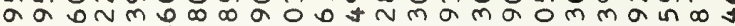

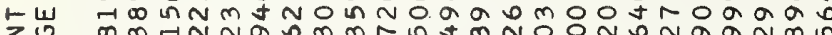

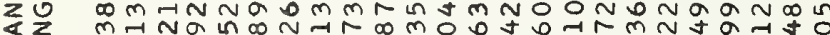
इ己 $\vec{n} \alpha$

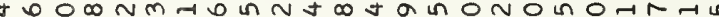

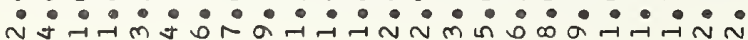

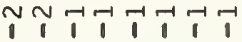

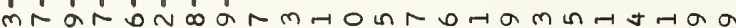
a

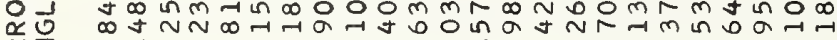

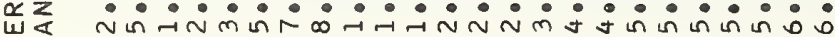

ITH

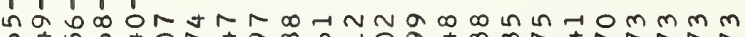

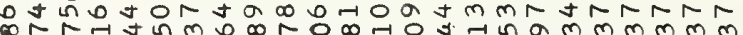

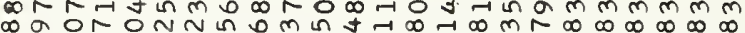

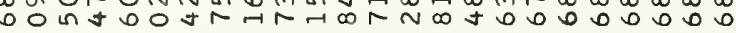

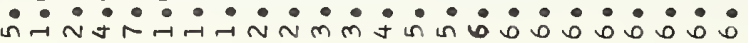

$\sum_{0}^{u}$

$\varangle \ll$

ज茪

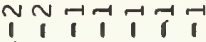

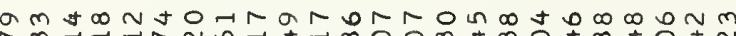

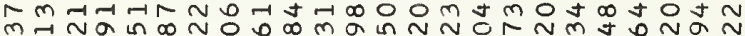

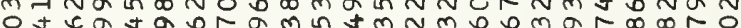

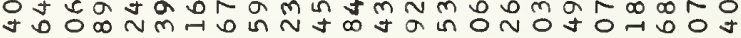

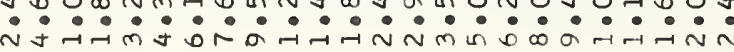

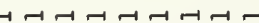

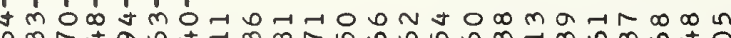
$m \sim$ in

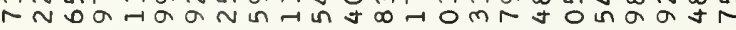

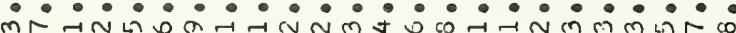
M̂

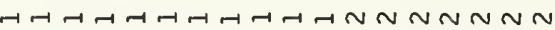

$\underset{⿱ ㇒}{ \pm}$

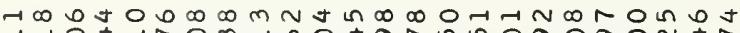
-1

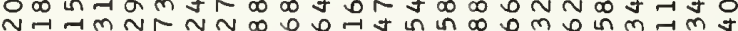
N

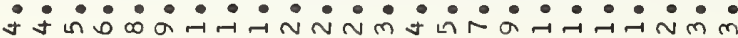

n

$\neg \neg \rightarrow \rightarrow \neg \sim$

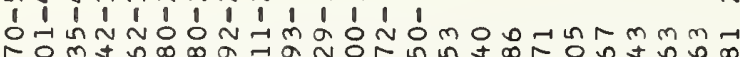

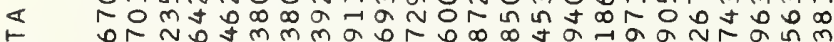
JI ẫ

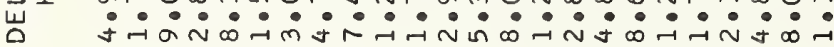
I

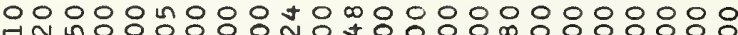

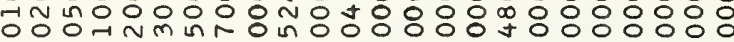

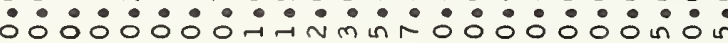

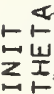

0000000.00000000000000000

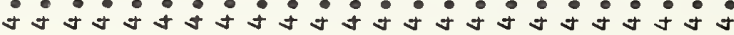

A t

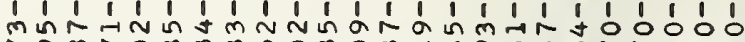

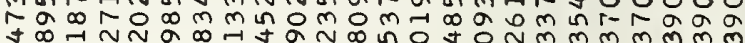

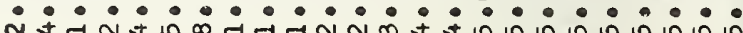

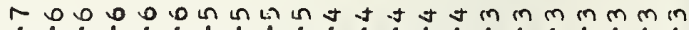
$\begin{array}{lllllllllllllllllllllll}0 & 1 & 1 & 1 & 1 & 1 & 1 & 1 & 1 & 1 & 1 & 1 & 1 & 1 & 1 & 1 & 1 & 1 & 1 & 1 & 1 & 1 & 1\end{array}$ 응ㅇㅇㅇㅇㅇㅇㅇㅇㅇㅇㅇㅇㅇㅇㅇㅇㅇㅇㅇㅇㅇㅇㅇㅇㅇㅇㅇㅇㅇㅇㅇㅇ의응응응응응 :은?

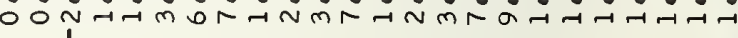

$\neg-\dashv-\dashv-N \sim N N N N N N \sim N m m m m m$

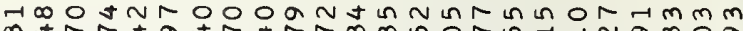
mJr No 0 i

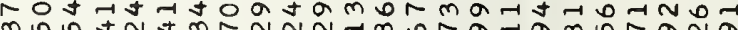

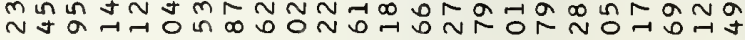
Na

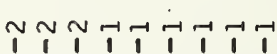

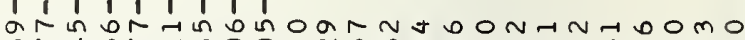
ming

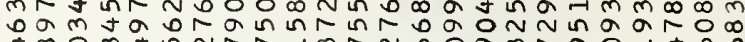
$\because \infty 0 m y n$ ?

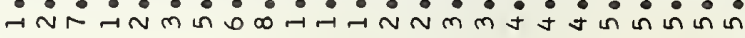

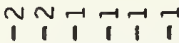

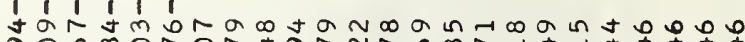

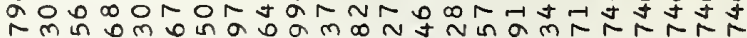
Năo

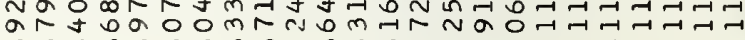

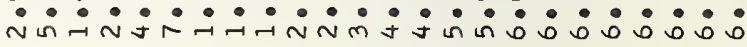

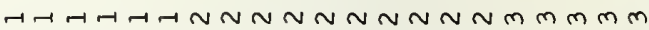
$m \sim N=\infty \sim O N \infty$ t Najป

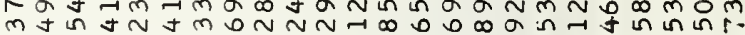

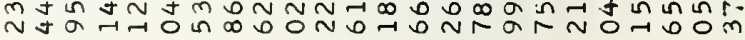

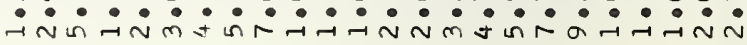

$\sim \sim \sim \rightarrow-\rightarrow \rightarrow-1$

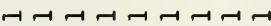

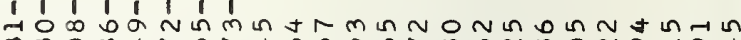

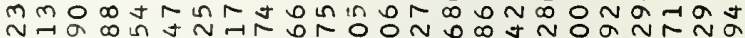
a

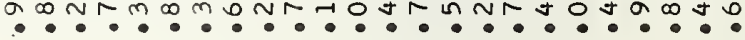
जी

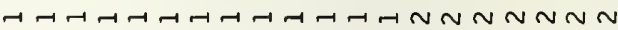

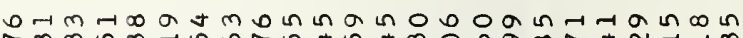

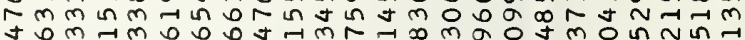
ปै

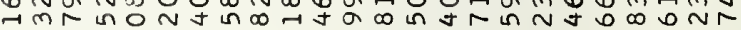

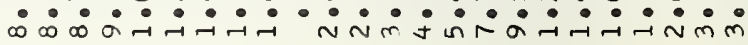

In

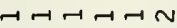

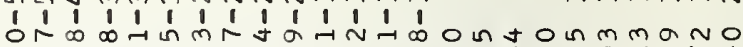

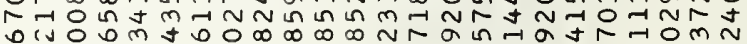
bu

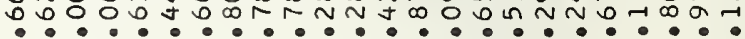

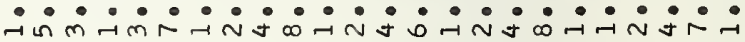

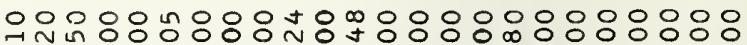

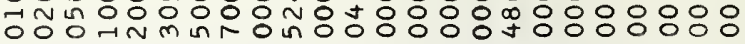

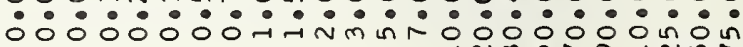

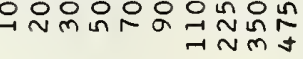

000000000000000000000000

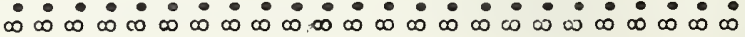

000000000000000000000000

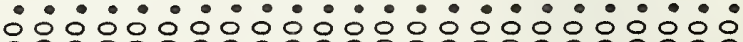
N 


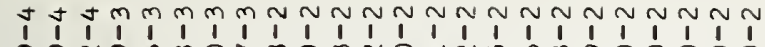

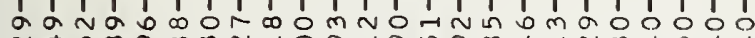

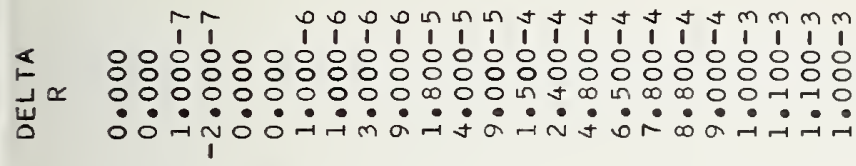
I

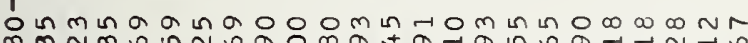

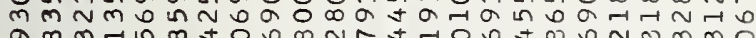

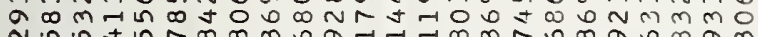

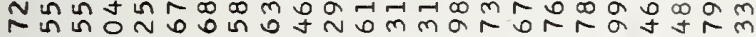

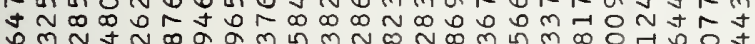

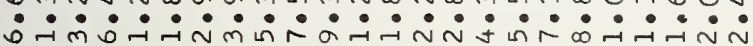

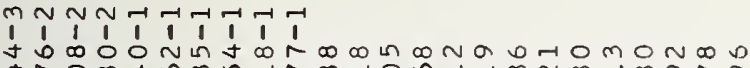

w t t 界

N N N

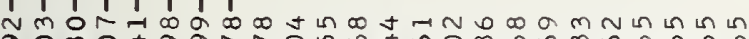

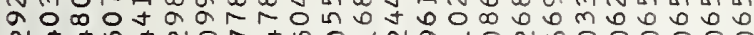

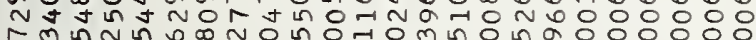

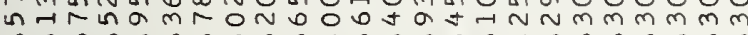

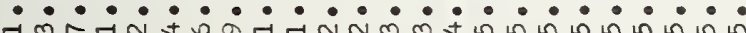

$\rightarrow$ -

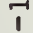

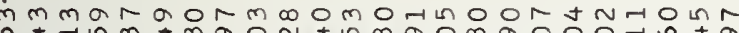
约

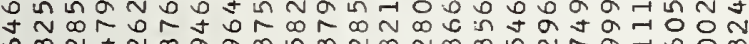

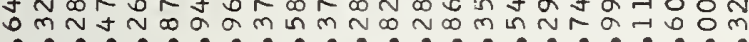

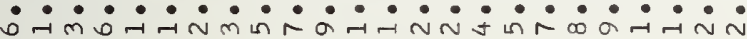

1 $N$ N

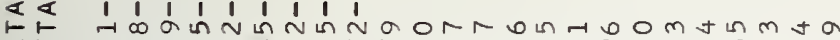
Jt mon O구의

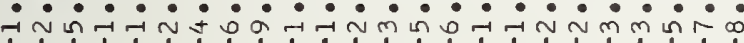

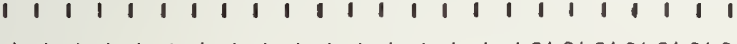

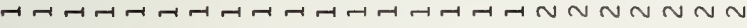

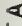
In in 0 t t m

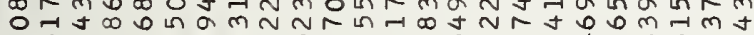

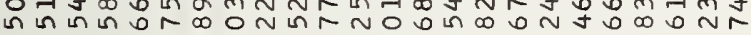

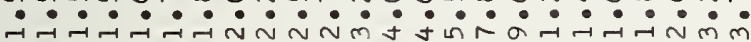

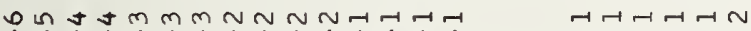

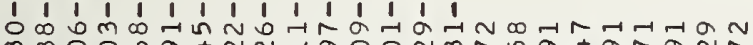
$\ll$

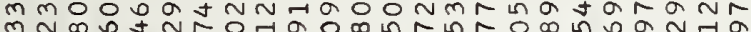

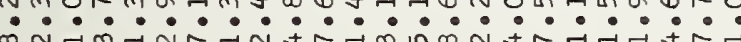

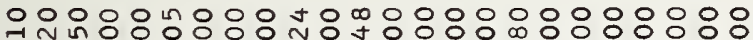

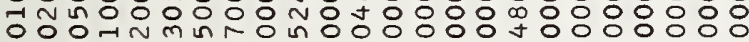

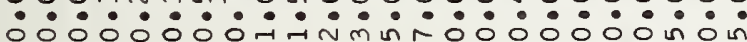
I

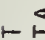

000000000000000000000000 ¿ in in in in in in in in in in in in in in in in in in in in in in in in

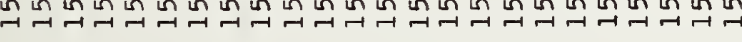

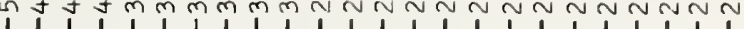

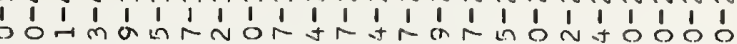

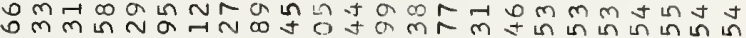

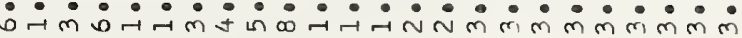

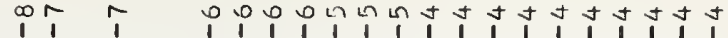

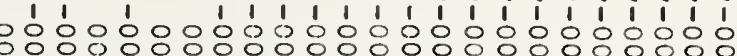

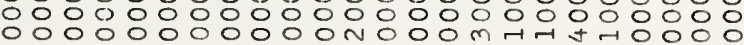

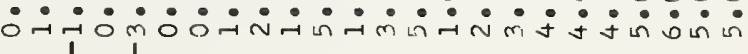

$\overrightarrow{1} \overrightarrow{1}$

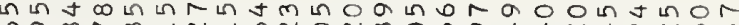

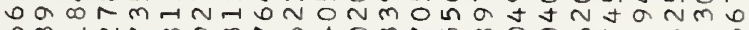

0m-1N

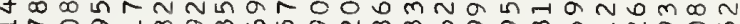
HROनल m心

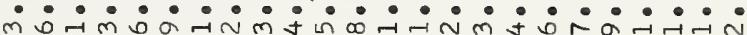

M

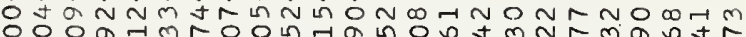

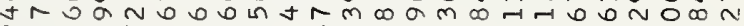

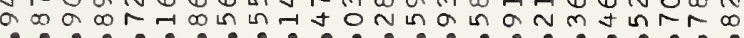

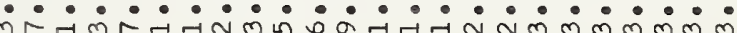

$m \sim N \sim \boldsymbol{H}^{-1}-1-1-1$

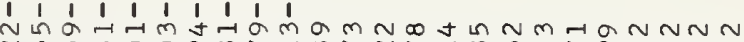

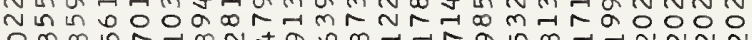

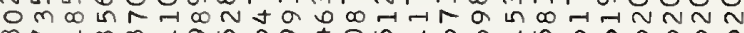

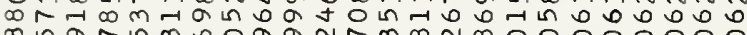

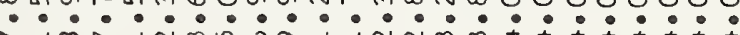

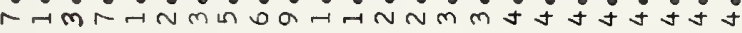

$1+1$

on

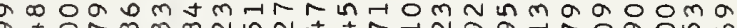
ator os mon m mbrm 0 ar

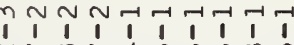

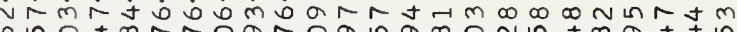

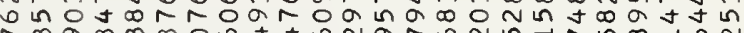

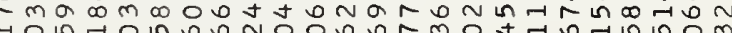
ㄴ? जी ન

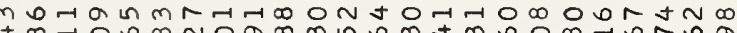

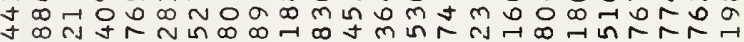
onja

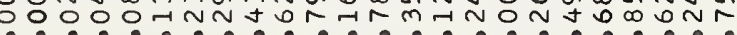
$\dot{m} \dot{m} \dot{m} \dot{m} \dot{m} \dot{m} \dot{m} \dot{m} \dot{j} \dot{j} \dot{j} \dot{i} \dot{0} \dot{\infty} \dot{-} \dot{-} \dot{i} \dot{-} \dot{i} \dot{N} \dot{m}$

i in intatimmmnnn-1

$\rightarrow-r-r$

I

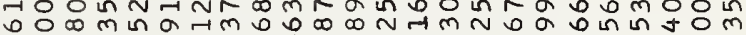

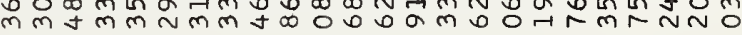

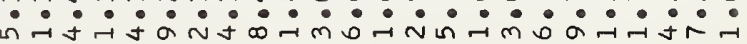

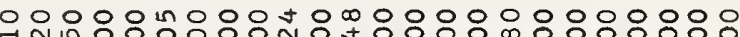

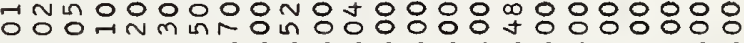

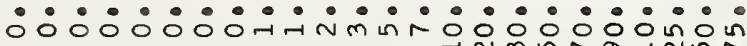
-

000000000000000000000000 $\dot{\circ} \dot{\circ} \dot{\mathrm{m}} \dot{\mathrm{m}} \dot{\mathrm{m}} \dot{\mathrm{m}} \dot{\mathrm{m}} \dot{\mathrm{m}} \dot{\mathrm{m}} \dot{\mathrm{m}} \dot{\mathrm{m}} \dot{\mathrm{m}} \dot{\mathrm{m}} \dot{\mathrm{O}} \dot{\mathrm{i}} \dot{\mathrm{m}} \dot{\mathrm{O}} \dot{\mathrm{m}} \dot{\mathrm{m}} \dot{\mathrm{m}}$

000000000000000000000000

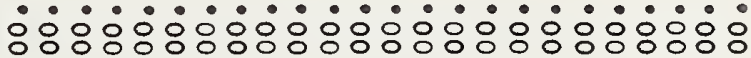

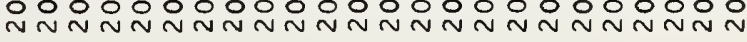




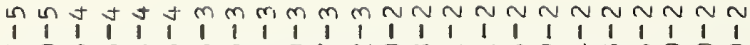

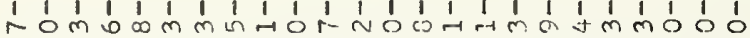
เU A. 0 -

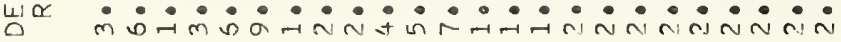

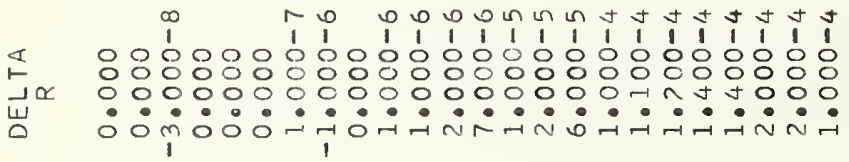

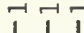

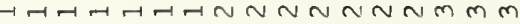

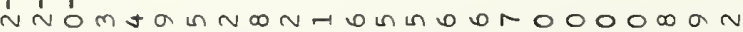

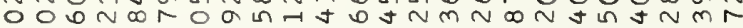

レய zo

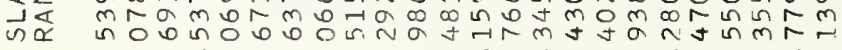

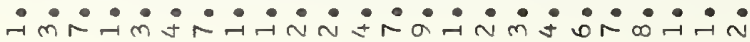

\section{$m m m \sim N \sim N-1-1-1-1$}

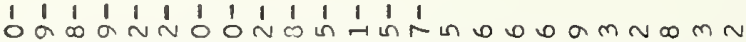
舀 o 点这

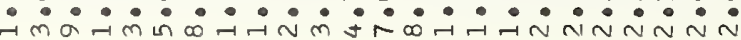

$m m \sim N-1-1-1-1$

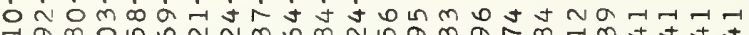

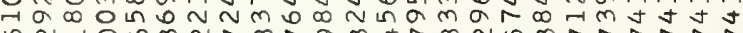
$\frac{2}{4}$

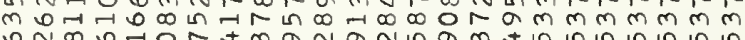
Jma va vinam

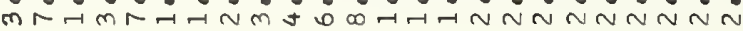

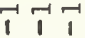

$\rightarrow r-r-r-N \sim N \sim N N \sim m m m$

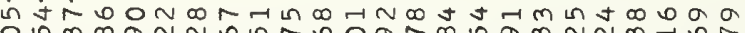

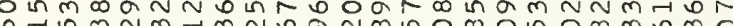

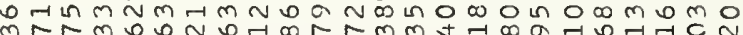
m

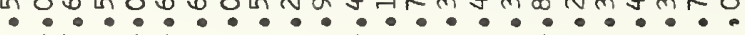

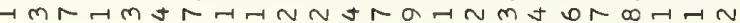

$m m \sim \sim N N-r-1-1$

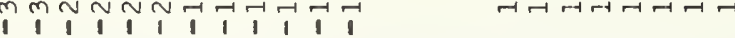

$\ll$

岀岌

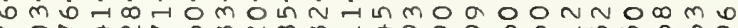
tor 0 N m $\infty$ a m

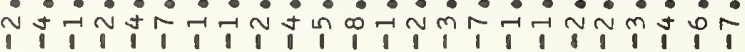

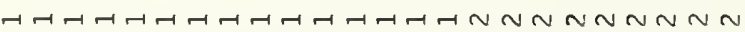

tantrao inm $N$ t t

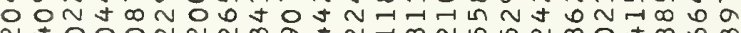

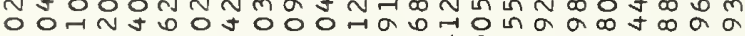

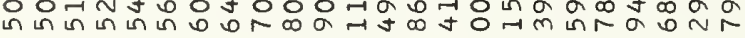

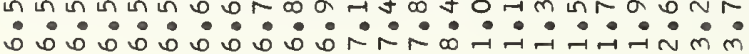

onn

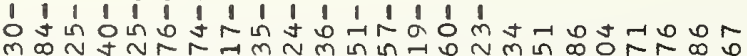

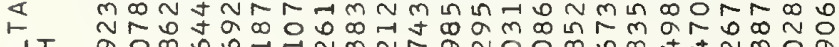

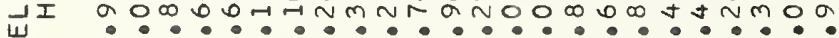

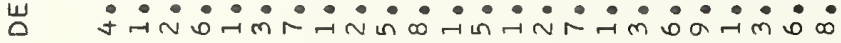

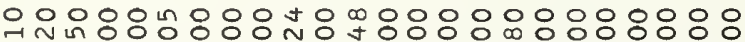

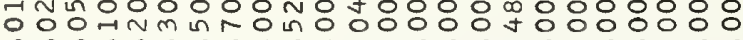

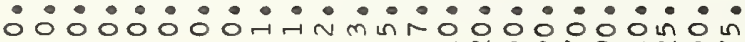
I

に吉

000000000000000000000000 的

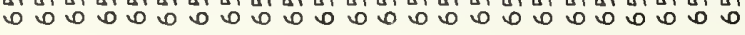

$\omega$

U $\quad 000000000000000000000000$ 崩 号 $\quad$ N
Ln m in th t t

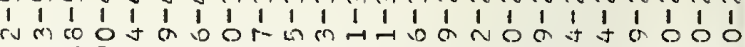

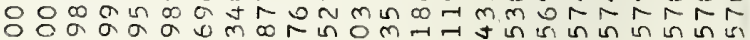

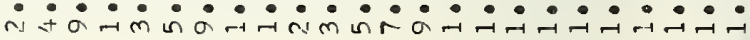

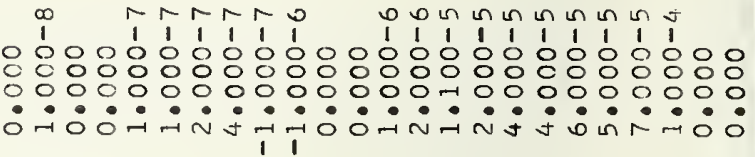
HनH in $00 n$ n

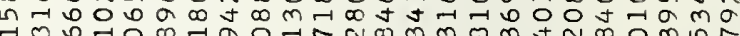
Im 0 -10 0 -

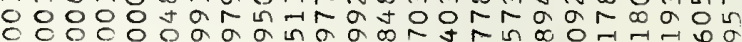

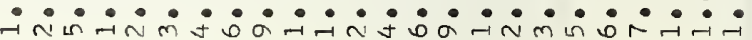

m

trmabrmomog on

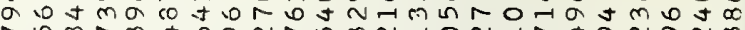

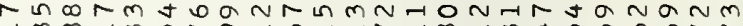
$\because m \infty \neg m$ เ

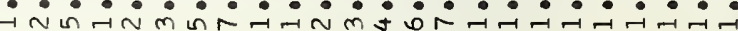

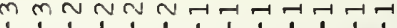

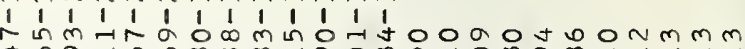
于 0 a

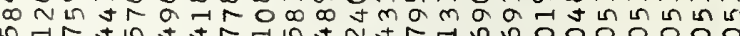
mก$\bullet \bullet \bullet \bullet \bullet \bullet \bullet \bullet \bullet \bullet \bullet \bullet \bullet \bullet \bullet \bullet \bullet \bullet \bullet \bullet \bullet \bullet$

NTH-1

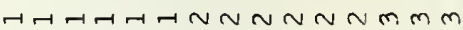

$\infty$ in to o o n m Nmmmmorl

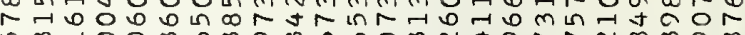
in moom

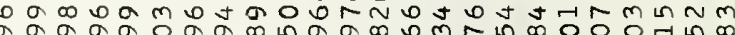

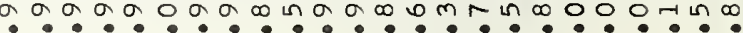

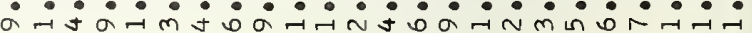

$m m m \sim N \sim N-1-1-1-1$

$\begin{array}{llllllllll}1 & 1 & 1 & 1 & 1 & 1 & 1 & 1 & 1 & 1\end{array}$

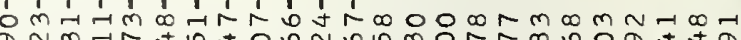
a ô

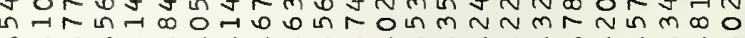
$? ?$ ก ?

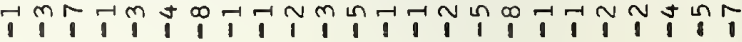

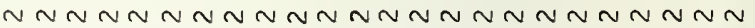
$m \infty 0 N$ In 궁ํำ

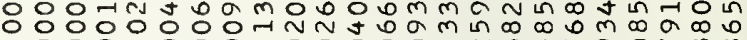

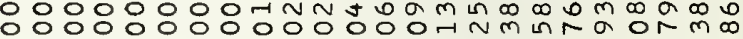
-

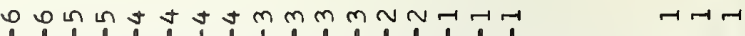
!́

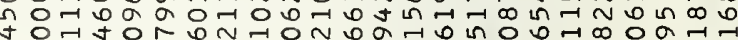

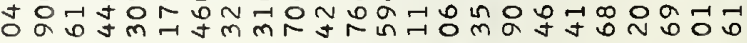

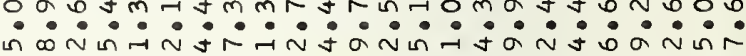

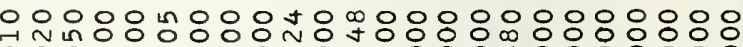

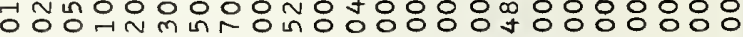

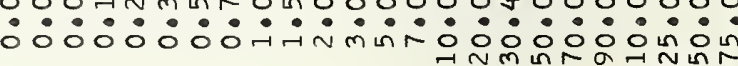

000000000000000000000000 O $\dot{0} \dot{0} \dot{0} \dot{0} \dot{0} \dot{0} \dot{0} \dot{0} \dot{0} \dot{0} \dot{0} \dot{0} \dot{0} \dot{0} \dot{0} \dot{0} \dot{0} \dot{0} \dot{0}$ 응ㅇㅇㅇㅇㅇㅇㅇㅇㅇㅇㅇㅇㅇㅇㅇㅇㅇㅇㅇㅇㅇㅇㅇㅇㅇㅇㅇㅇㅇㅇㅇㅇㅇㅇㅇㅇㅇㅇㅇㅇㅇㅇㅇㅇㅇㅇㅇㅇㅇㅇ음

000000000000000000000000

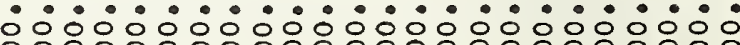
N N N N N N N N N N N N N N N N N N N N 


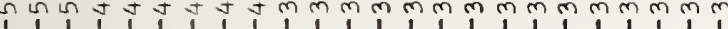
in $\sim 200 \mathrm{a}$ a

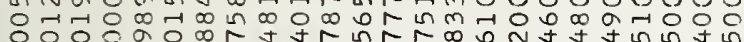
. ? ?

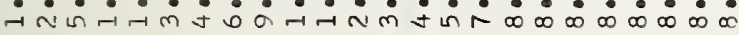

$\infty \infty$

rit

$\circ$

$\begin{array}{llll}1 & 1 & 1\end{array}$

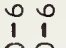

in in in in in

$\varangle$ $\vec{u}^{\infty}$

$\circ 800000000000000000000$

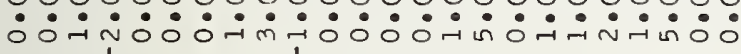

N $\overrightarrow{1} \overrightarrow{1} \overrightarrow{1}$

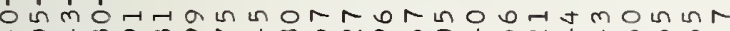

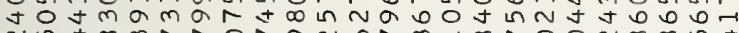
-

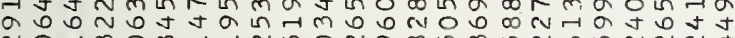

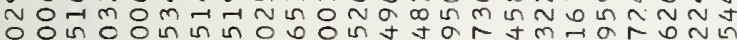

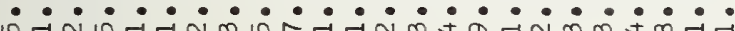

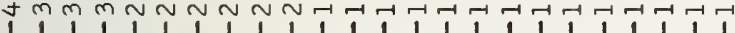

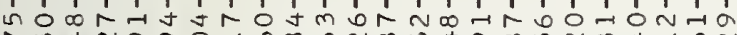

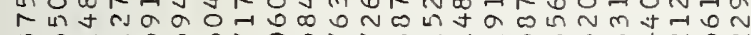

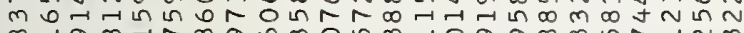

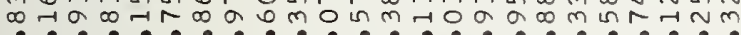

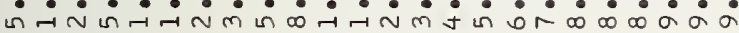

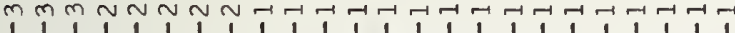

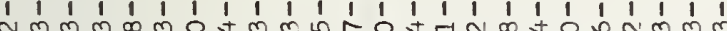
$+\infty a \omega_{0} 0$ in

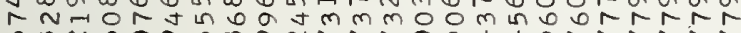

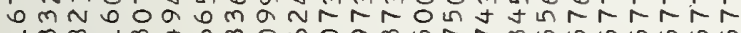

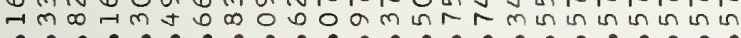
-

IT

-

岂

a bo a a N O o or omarthrtmro in OrNmtatot

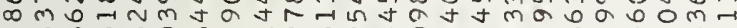

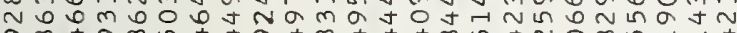

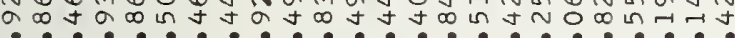

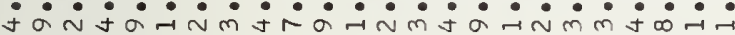

ammmenturn

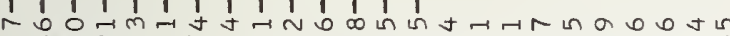

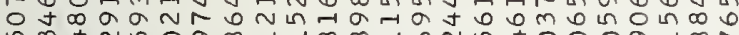

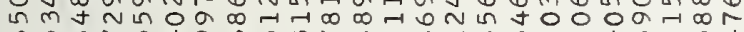

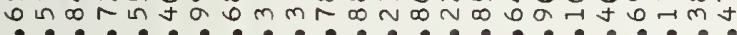

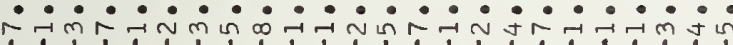

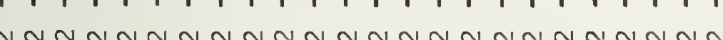
$0 m \sim i n-0 N N m+\infty 0 R m R \infty N m a \infty \infty a m$ Oनm

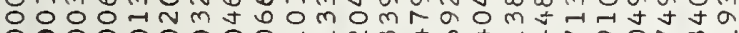

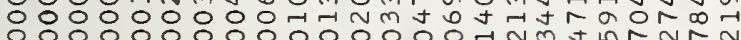

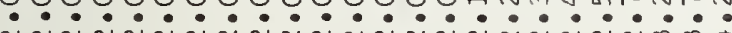

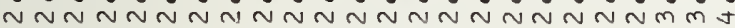

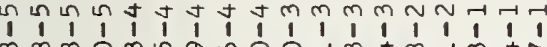

$\rightarrow r-$

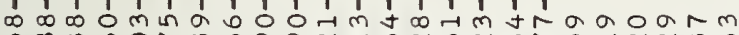
น ma

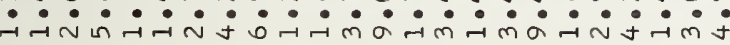

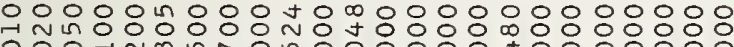
$000-1 n m$ in 0 in $000000 \% 0000000$

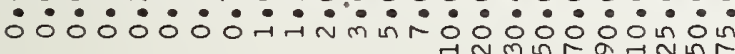
$\rightarrow N m$ in



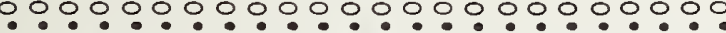

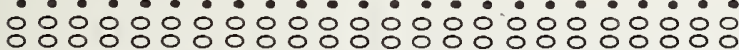

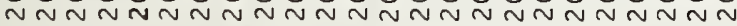

000000000000000000000000

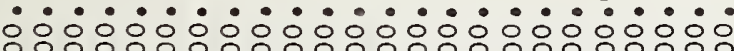

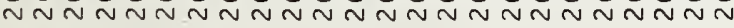

Whnm

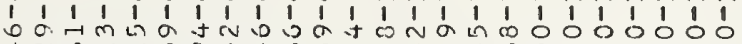
t

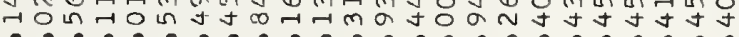
(i)

$\infty \infty \infty \infty \infty \hat{N}-\hat{N}$

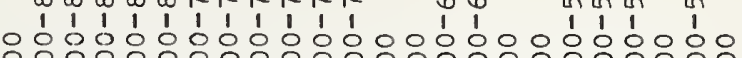

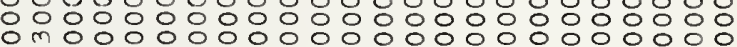

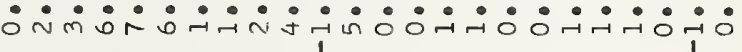

$\hat{1} \hat{\imath} \vec{\imath} \vec{\imath} \vec{\imath}$

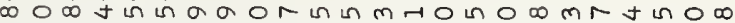
$\rightarrow a n 0 \infty 0$ in $\rightarrow 0 m+0 N 0$ and $\infty \rightarrow m-r O r-\infty N N$ J 0 寸a

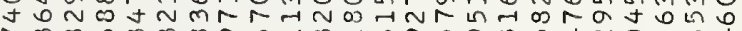

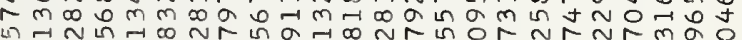

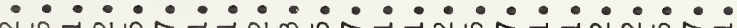

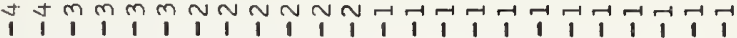

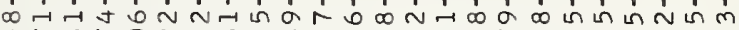
in

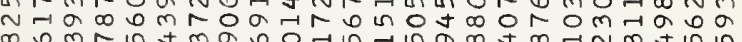
$\infty \because m ?$ เก $N$ II $N$ In

†

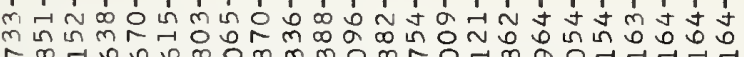

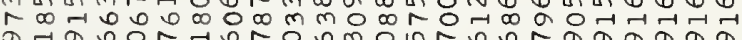

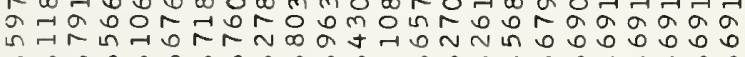

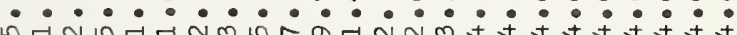

în

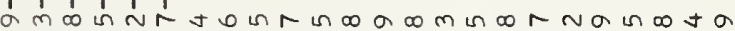

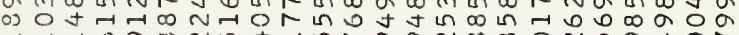

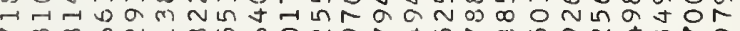

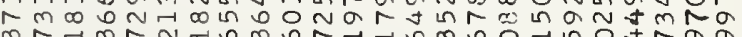

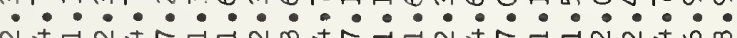

A

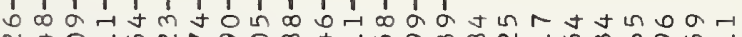
vง ODtrR $\because ?$ ? ? ? ? ? ? ? ? ?

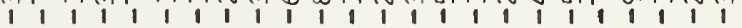

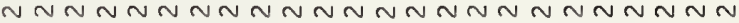

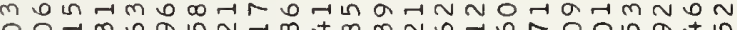
이의

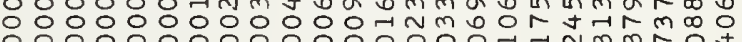

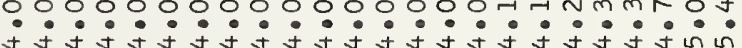

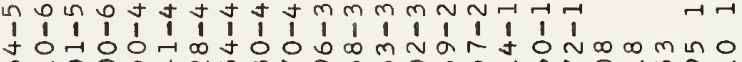
次。

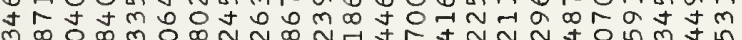

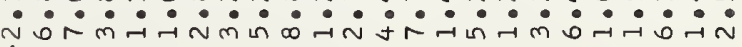
1

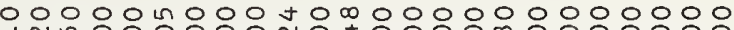
등에

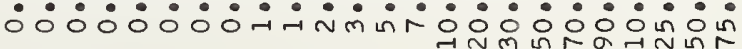
$\rightarrow \sim m+$

000000000000000000000000

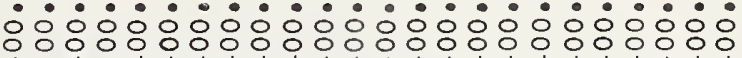

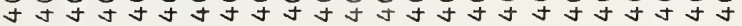

000000000000000000000000

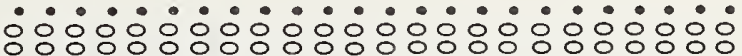

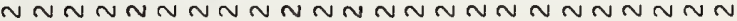



$\vec{\omega}$

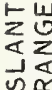

$\propto w$

$\frac{\alpha}{\alpha}=$

2

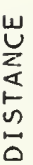

$<<$

占

WI

$\frac{1}{I}$

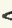

WI

$\underset{I}{I}$

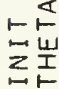

崩

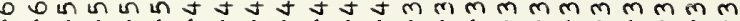
$\begin{array}{llllllllllllllllllllllll}1 & 1 & 1 & 1 & 1 & 1 & 1 & 1 & 1 & 1 & 1 & 1 & 1 & 1 & 1 & 1 & 1 & 1 & 1 & 1 & 1 & 1 & 1 & 1\end{array}$

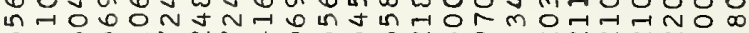

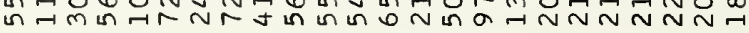

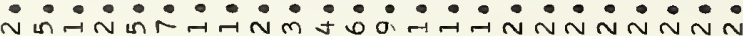

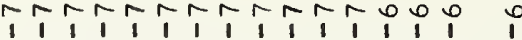

in

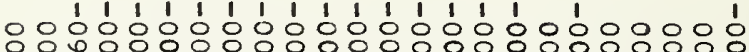

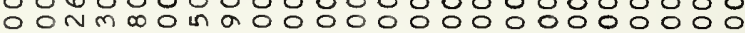
:

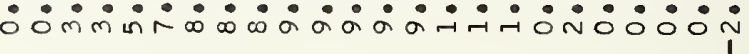

Niञ $\neg-\sqcap \sqcap \sqcap N N N N$ N N 1 in

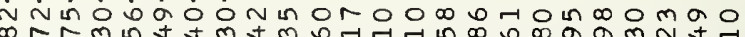
$\infty \pi r m$ i t

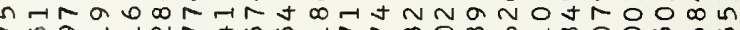

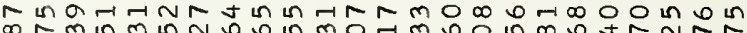
$\infty r m$ in $m$ in $N \omega$ in $m 0-1 m$ o 0 in

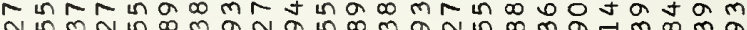
- $:-\circ \cdot \cdots$

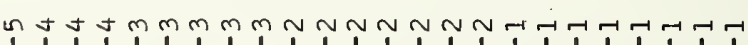

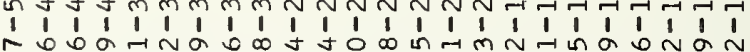
No

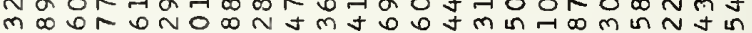

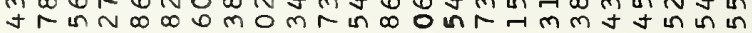
m

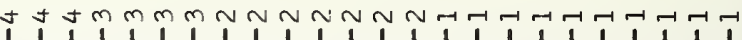

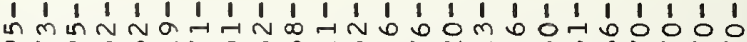

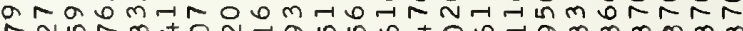
- N n $r m$ to $N$ a n N - mám

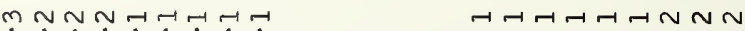

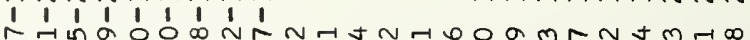

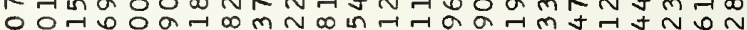

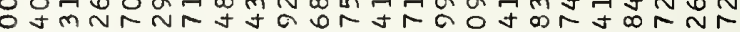

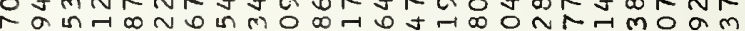

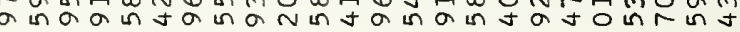

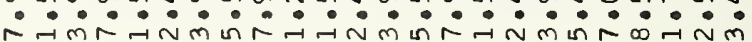

A4tmmm

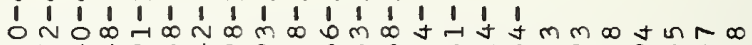
r t tain

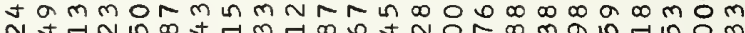

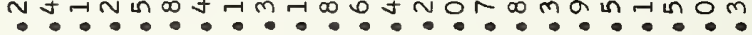

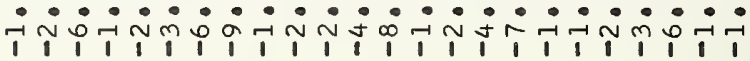

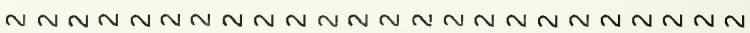

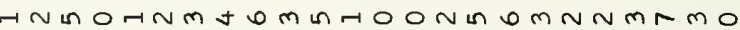

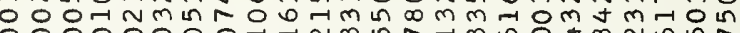

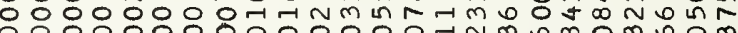
$00000008000000 \mathrm{Nm} 000 \mathrm{~m} 0 \mathrm{~m}$ $0000000000000000000 \pi n$ n

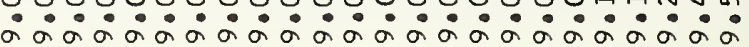

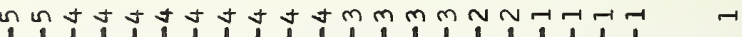

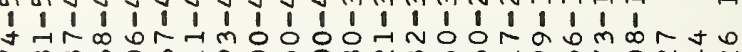

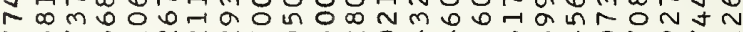

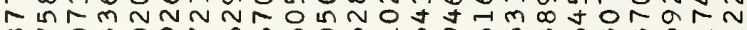

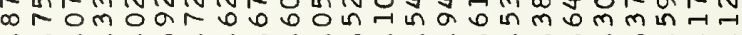

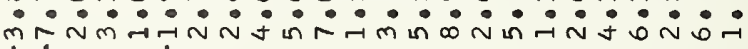
11

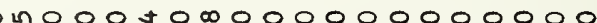
-1
0

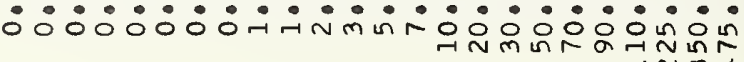
$\rightarrow \sim m+$

000000000000000000000000

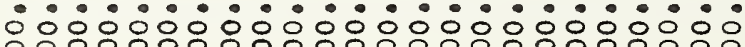
a

- 00000000000000000000000 z z 0 0 0 0

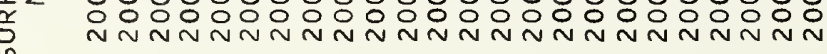


$\leftarrow$ $5 \alpha$ w

\section{a}

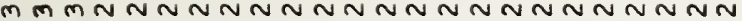

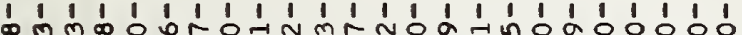
añon

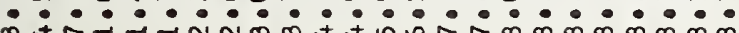

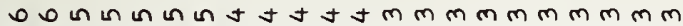

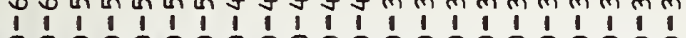

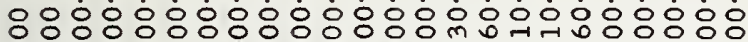

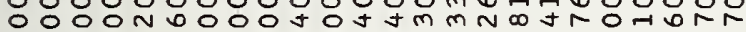

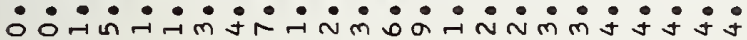

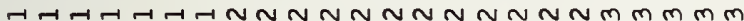
oromoun on 0 in

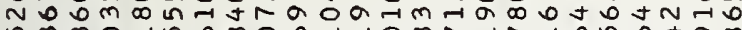

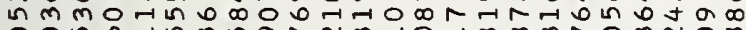
은 的分 뉴 ก!

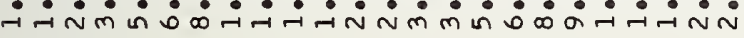

-1

m thommnOmoOtrm

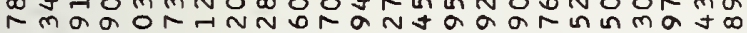

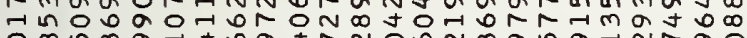

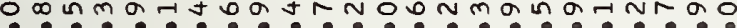

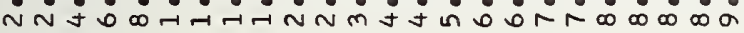

-1

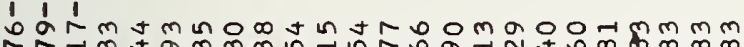
in in

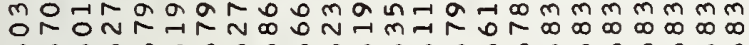
? ? ? ? $\cdots \cdots \cdots$

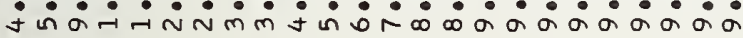

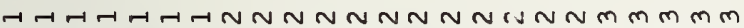

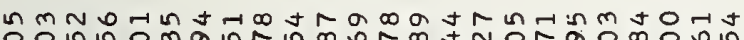

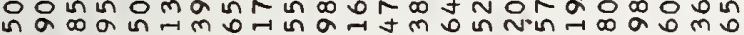

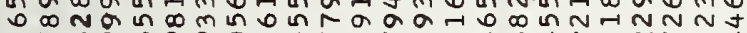
N

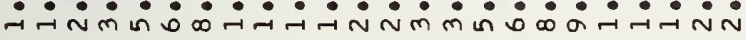

N

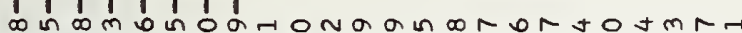

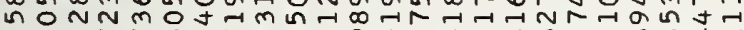

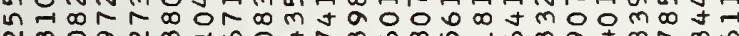
Nmog

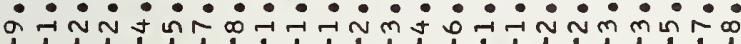

-

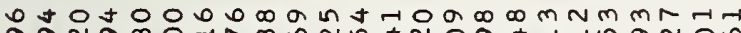
a

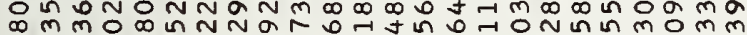

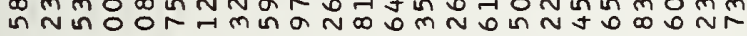

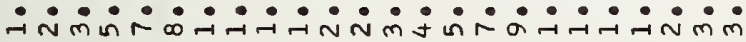

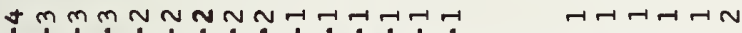

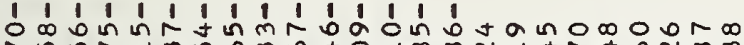
Nm n

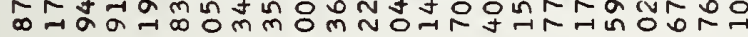

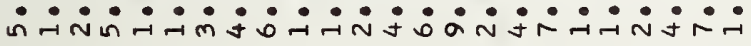

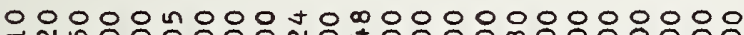
는

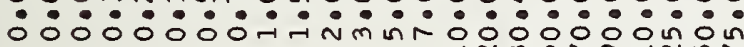

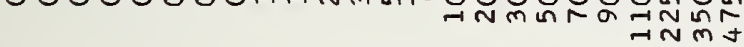

4000000000000000000000000

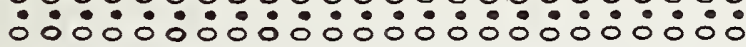

$\frac{u}{\alpha}$

a a a a a a a a a a a a a a a a a a a a N

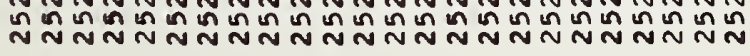

mmm $m$ m N N N N N

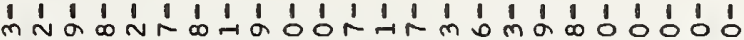

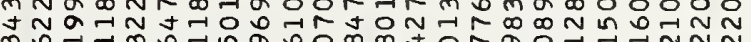
mo

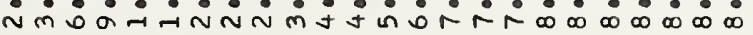

O O On氏

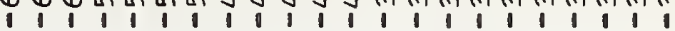

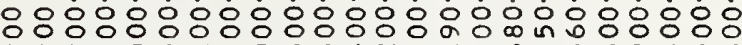

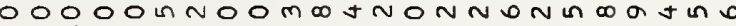

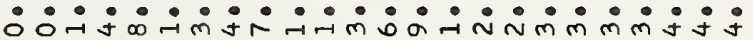

$\neg-\neg-7-N \sim N N N N N \sim N N N N m m m m m$

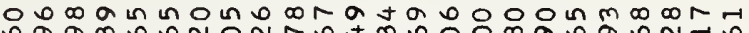
Nmóa

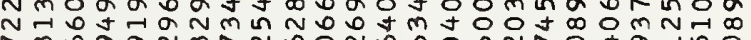
Nmo a

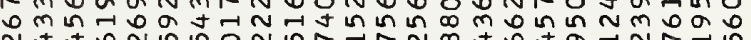
ก.?

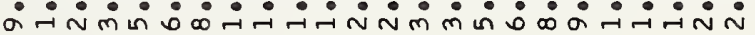

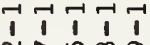

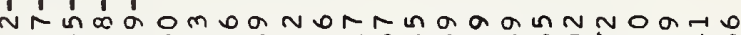

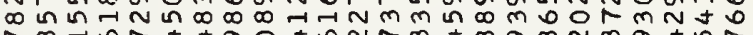

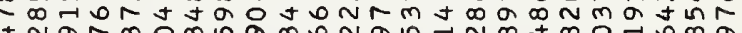

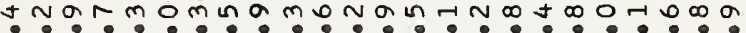

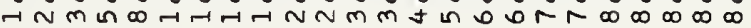

17

Nom t

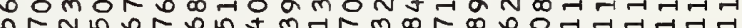
a in

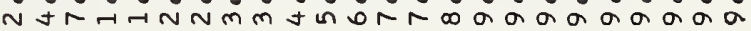

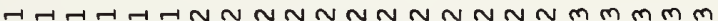

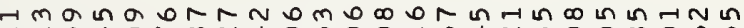

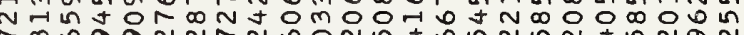
m o a a N N N Na ก.7.

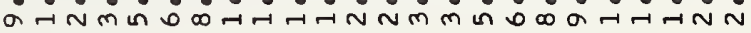

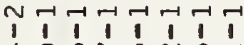

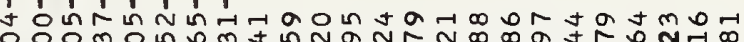

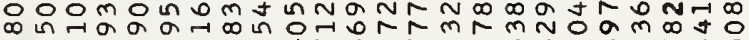
م

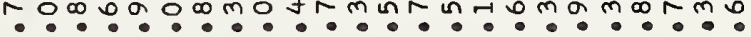

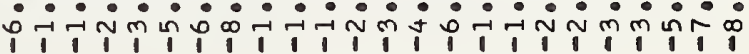
H-

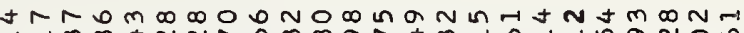

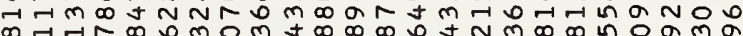

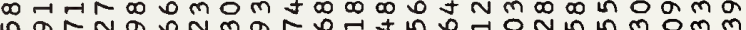
设

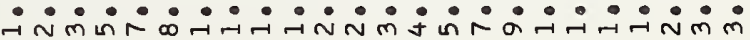

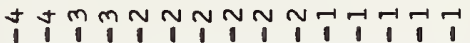

$\neg \sqcap-\neg \sqcap N$

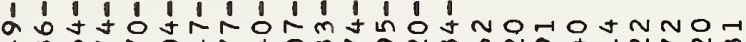

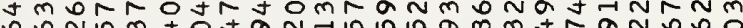

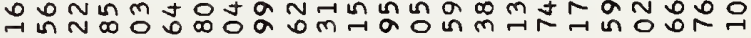

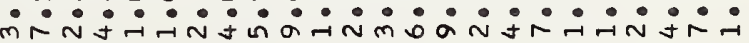

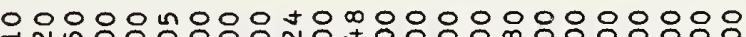

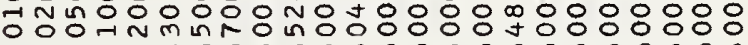

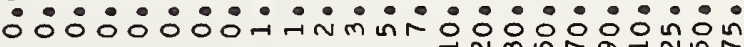
HNm

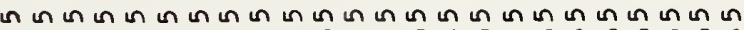
;

a a a a a a a a a a a a a a a a a a a a a a N

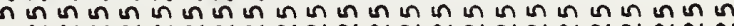

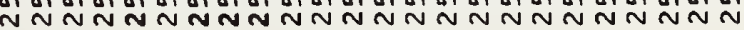




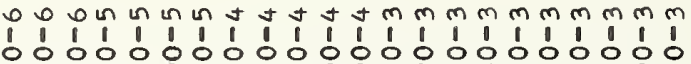

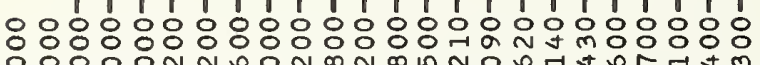
ㅇํㅇำ

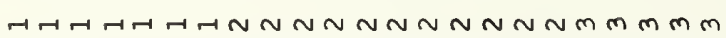
n $m$ n t o 上U

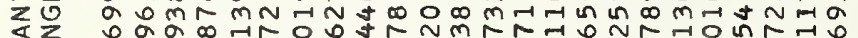

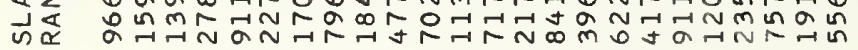

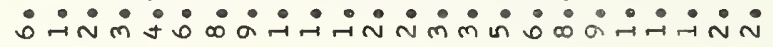

Нூ

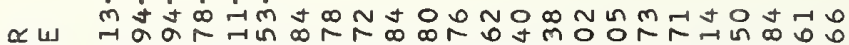
걱

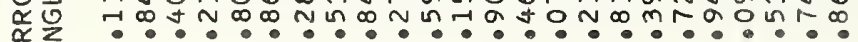
w

\section{0}

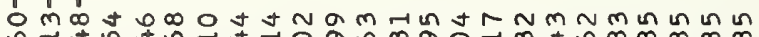
N

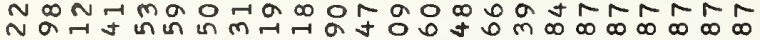

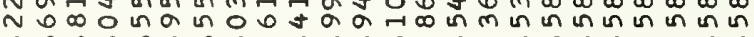

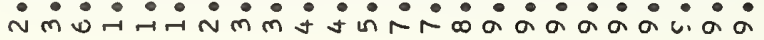

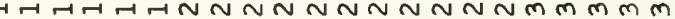
m n ăm

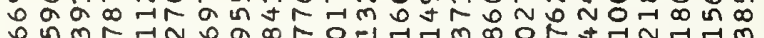

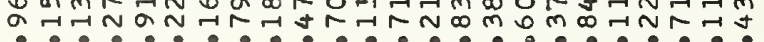

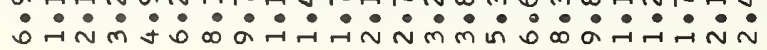

$\stackrel{\leftrightarrow}{\leftarrow \leftarrow}$

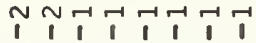

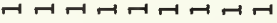

岕岌

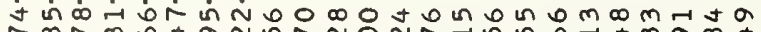
ror

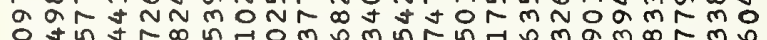

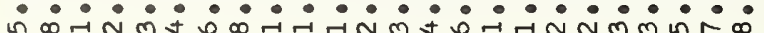
1 คー

r

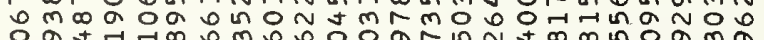

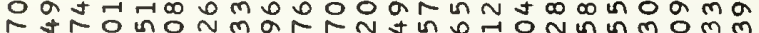
w

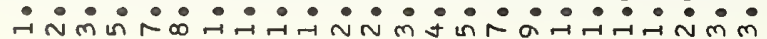

t

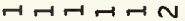

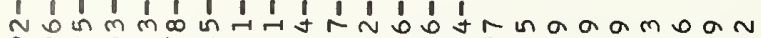
$\alpha a N$ in

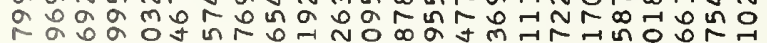
-

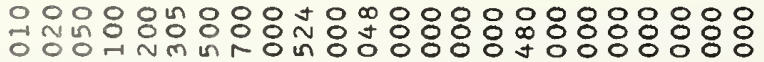

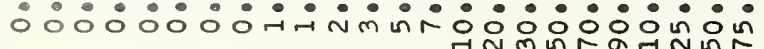

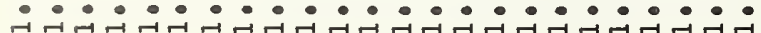

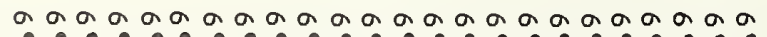

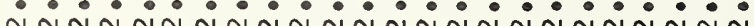

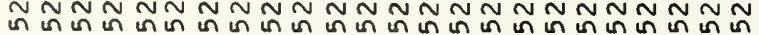

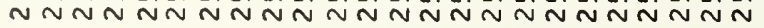

mmmm

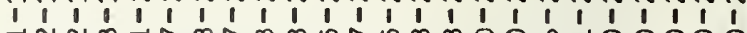

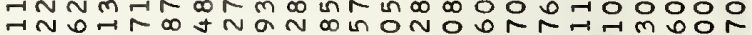
ror $\infty$ om $m$ n

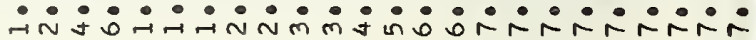

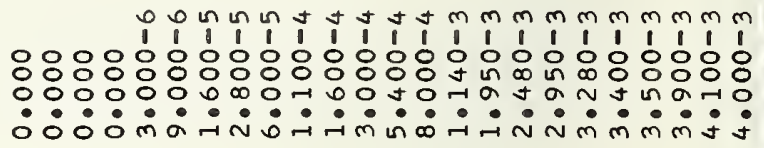
HATH-HNNNNNNNNNNNmmmmm TIn 0 NO O in in in $N m$ toj

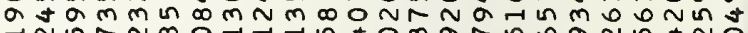

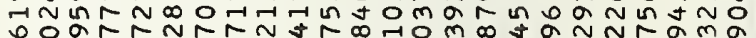

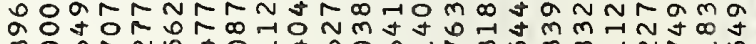
moviN

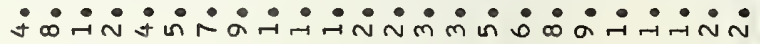

$\operatorname{lin},-1,1$

ḿm 1 m 1 ' m N trNO $N$ O $N$ N $n$ in

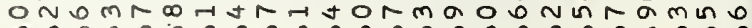

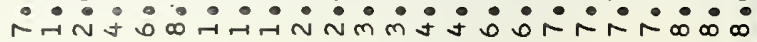

$\overrightarrow{1} \overrightarrow{1} \overrightarrow{1}$

ot o ondmmma

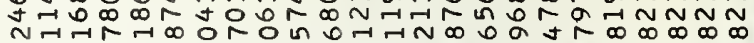

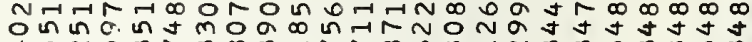

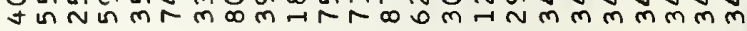

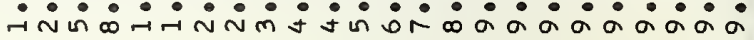

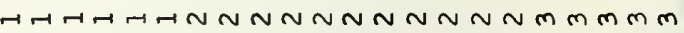

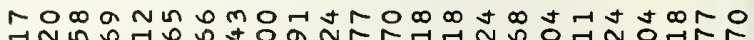
Tn

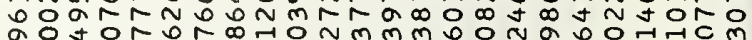
mogrn

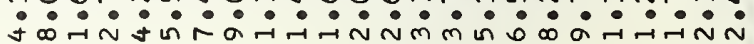

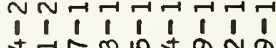

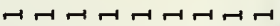

ปส

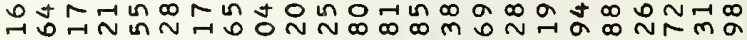

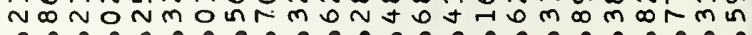

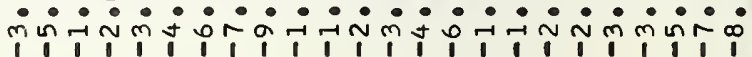

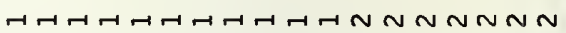

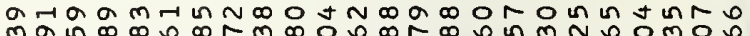

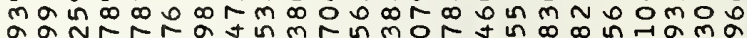

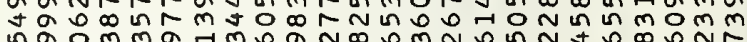

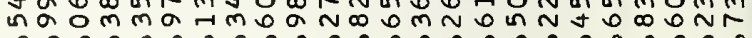

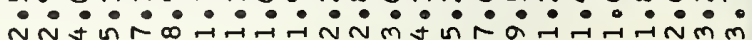

nงmm $m \sim N N N F-17-1$

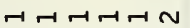

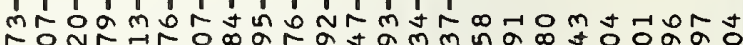

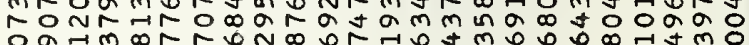

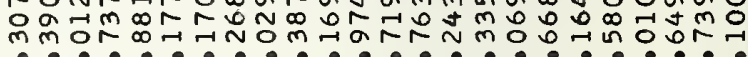

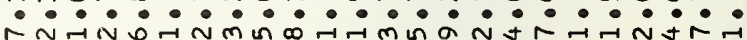

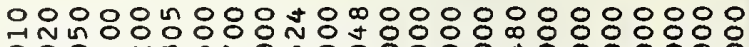

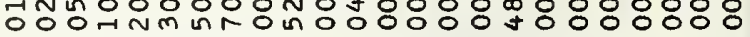

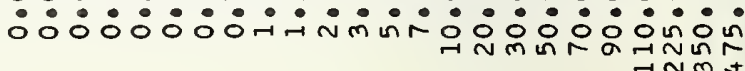

000000000000000000000000

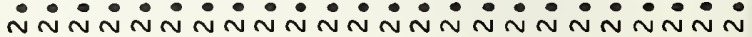

a a a a a a a a a a a a a a a a a a

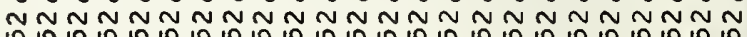

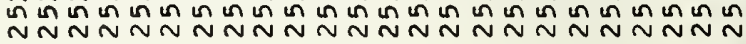



onoth ân

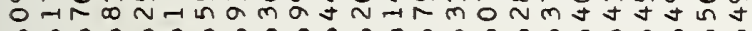

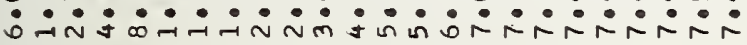

- 0 o

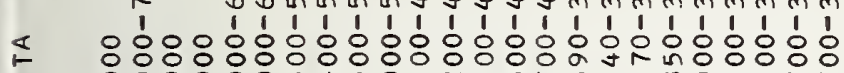

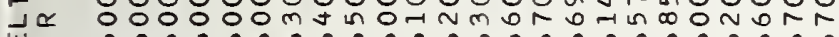

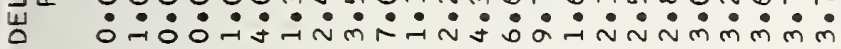

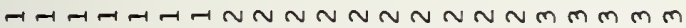

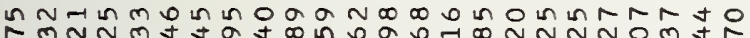
$\sim m N N m$ t

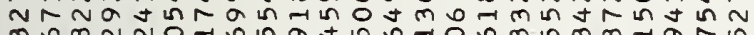

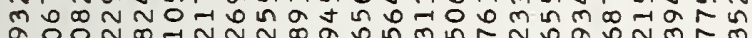

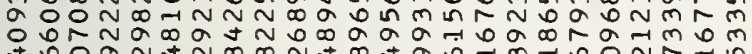

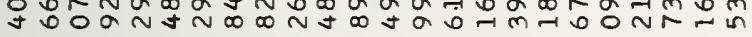

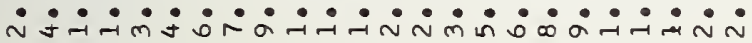

$\begin{array}{lllllll}1 & 1 & 1 & 1 & 1 & 1 & 1\end{array}$

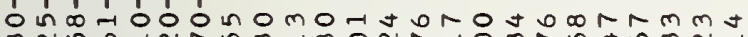

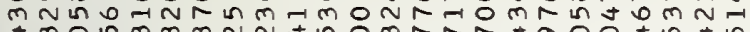
† mo in $m \infty N$ N

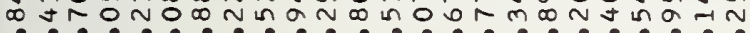

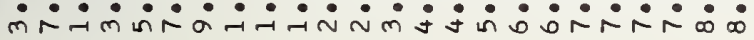

Ni

ก ก -1

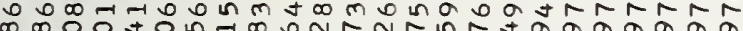

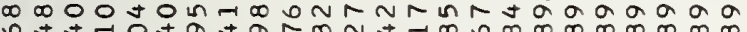
-

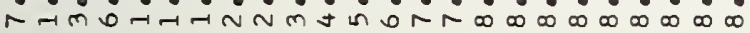

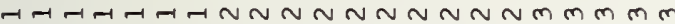

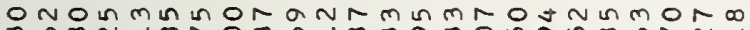

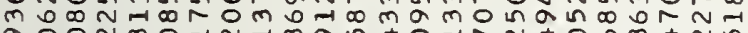

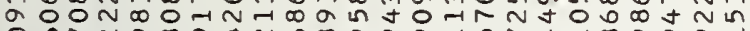
0 人

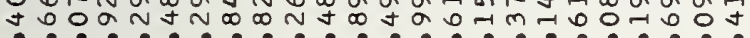

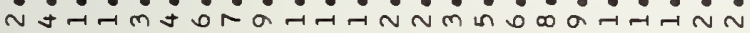

NNN-1-1-1 $N-1$

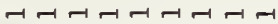

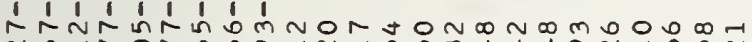
No-1

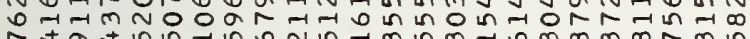
-

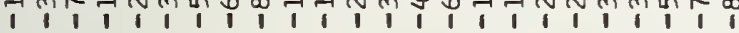

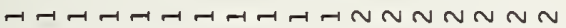

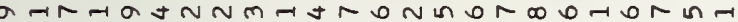

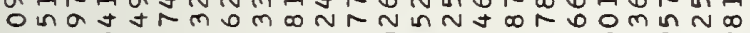

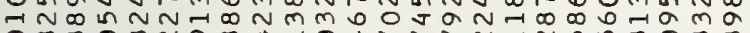

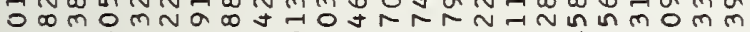

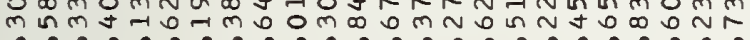

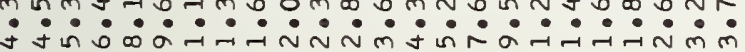

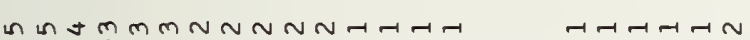

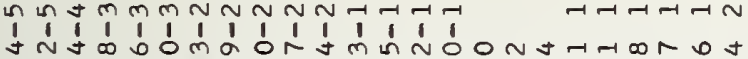

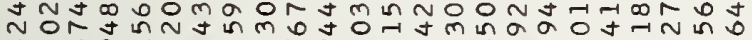

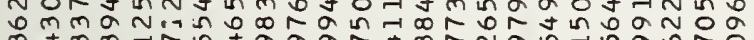
mงmm-ringa a No+rar-inm

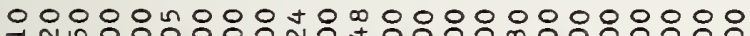

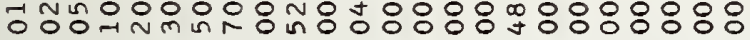
i

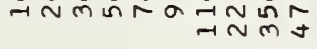

000000000000000000000000

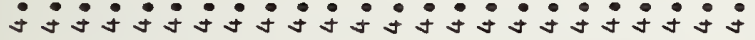

a a a a a a a a a a a a a a a a a a a a

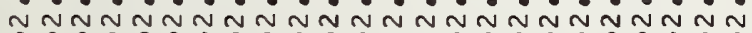

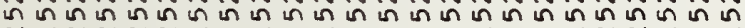

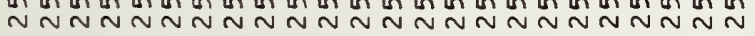

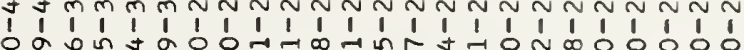

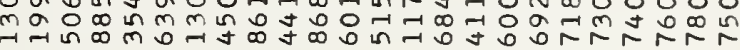

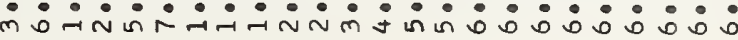

○

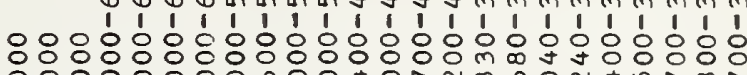

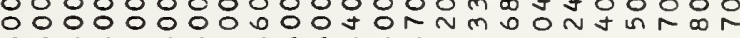

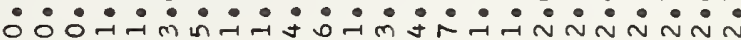

$\rightarrow-\rightarrow-\sqcap-N \sim N \sim N \sim N \sim \sim N m m m$

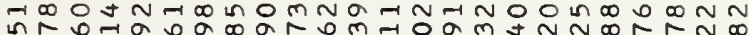

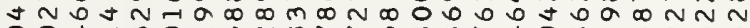

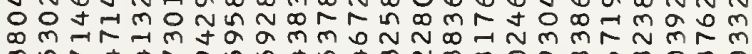
$m$ in $\pi$ t 10 in

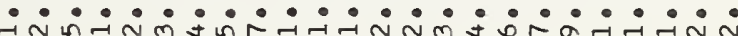

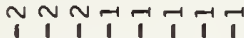

rNa-1

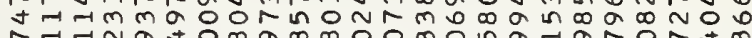

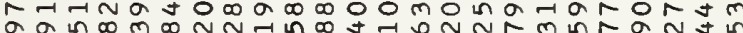
? - mám

N $N-1-1$

$1,117 \%$

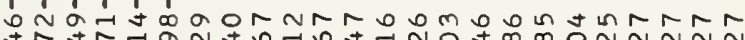

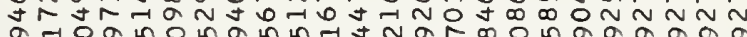
a

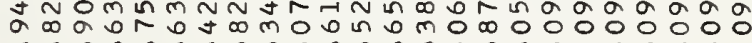

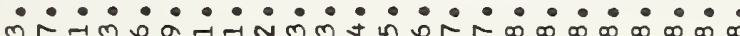

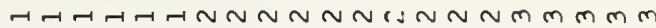

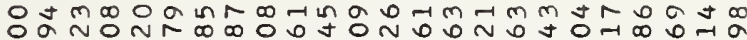

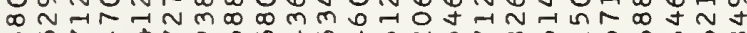
$m$ in $r+\neq N a$ in

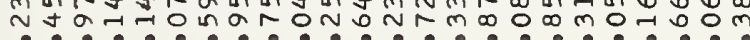

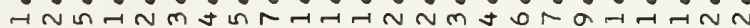

$m \sim N \sim-1-17-1$

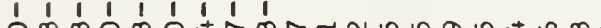

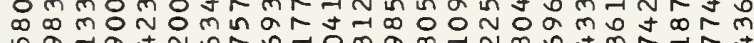
ina 0 -

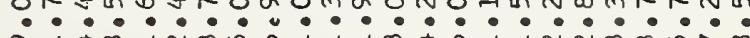

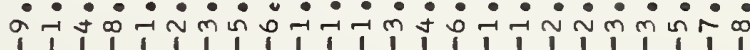

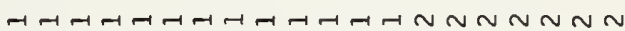

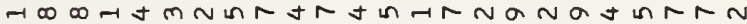

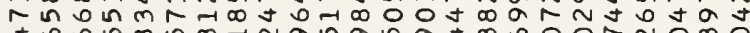

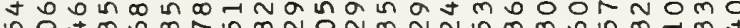

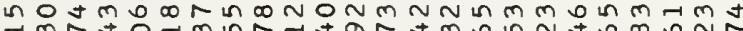
m

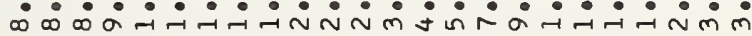

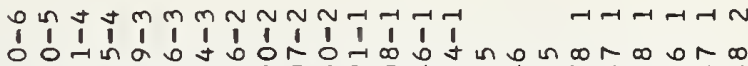

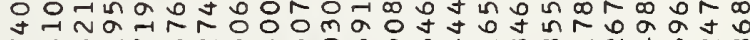
$r \infty m \sim N \infty N$ N a 0 thr 0 J

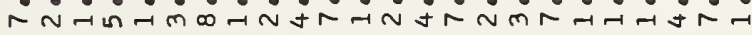

00000 m 000 tomo00000000000

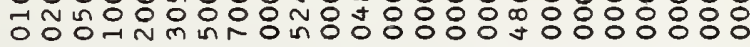

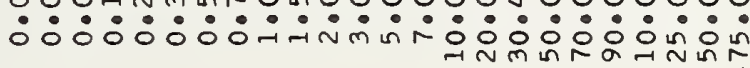

000000000000000000000000

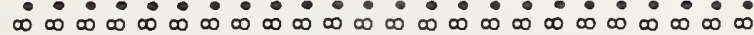

a a a a a a a a a a a a a a a a a a a

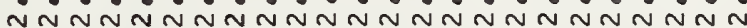

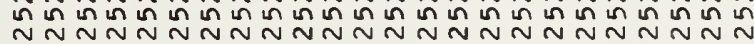




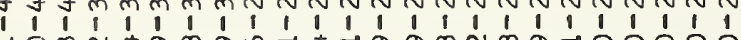

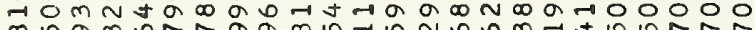

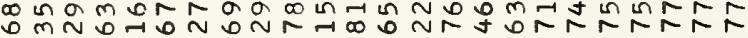

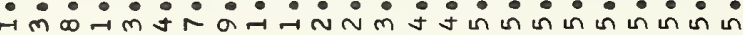

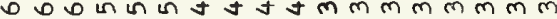

$\begin{array}{llllllllllllllllll}1 & 1 & 1 & 1 & 1 & 1 & 1 & 1 & 1 & 1 & 1 & 1 & 1 & 1 & 1 & 1 & 1\end{array}$

motomontom nn NRUam in 央施

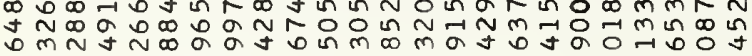

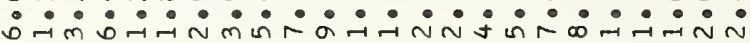

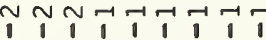

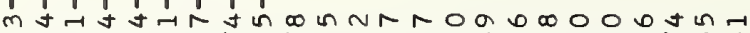
Q O $D-1 m m$ ONmOJ

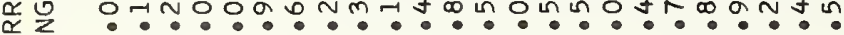
崖

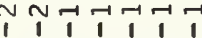

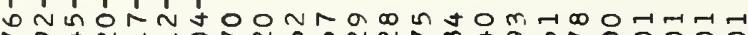
rath

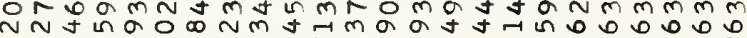
tNoOa a

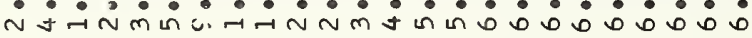

1.

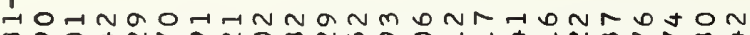
क

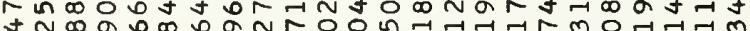

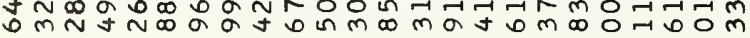

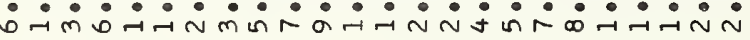

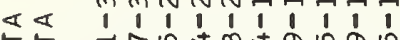

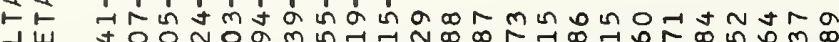

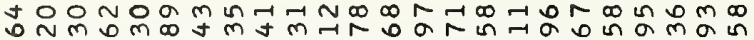

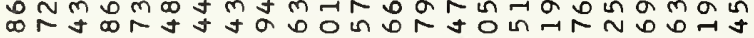

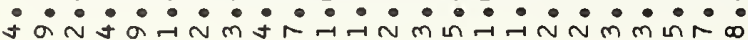

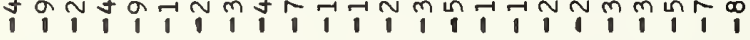
નન-નનન-નન-નન-નન-નળ

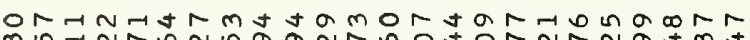

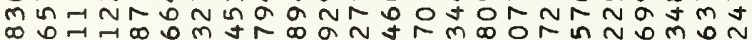

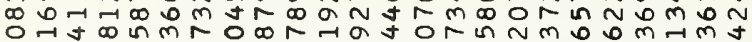

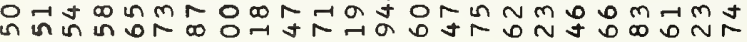

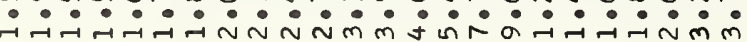

oun

$\neg \rightarrow-\rightarrow-n$

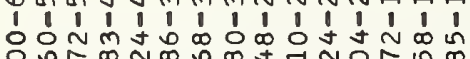

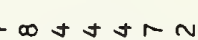

$\varangle$

$\stackrel{H}{H} I$ 等

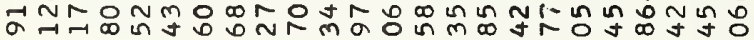

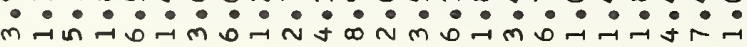
$\underset{I}{I}$

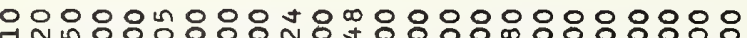

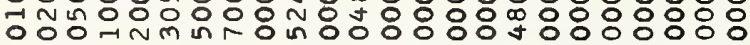

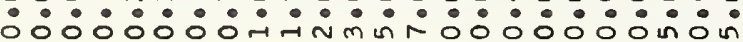

000000000000000000000000

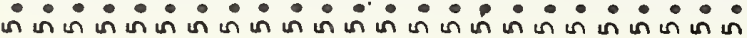

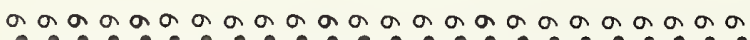
$\mathbb{Z}=\dot{\sim} \dot{\sim} \dot{\sim} \dot{\sim} \dot{\sim} \dot{\sim} \dot{\sim} \dot{\sim} \dot{\sim} \dot{\sim} \dot{\sim} \dot{\sim} \dot{\sim} \dot{\sim} \dot{\sim} \dot{\sim} \dot{\sim} \dot{\sim} \dot{\sim}$ 崩 的

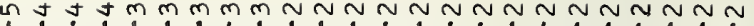

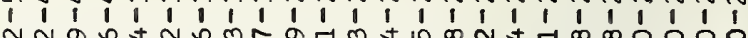

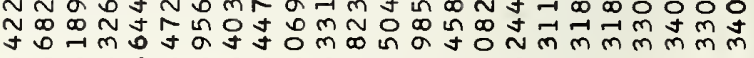

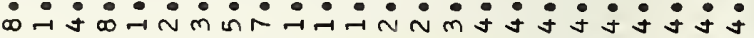

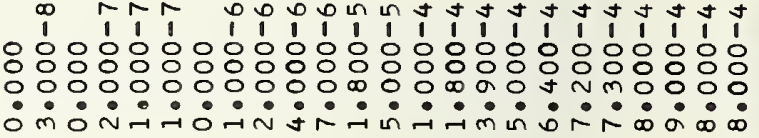
17 $\neg-\rightarrow-r-1 N N N N N N N m m m m$

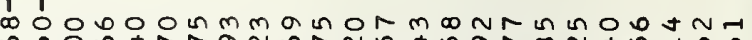
0 0 0 D t t

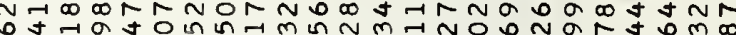

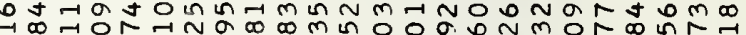

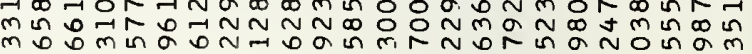

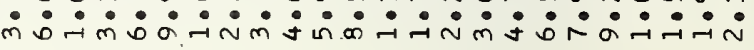

$m \sim N \sim-1-1-1-1$

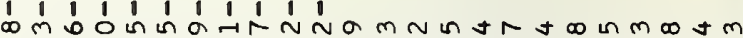

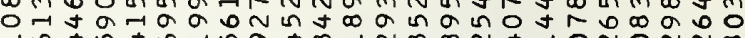

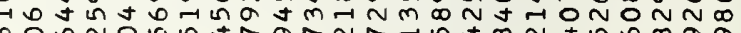
$m \circ 0 N O$ in

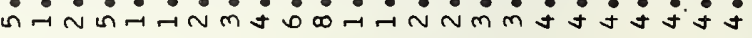

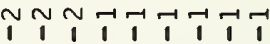

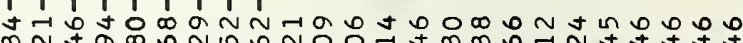

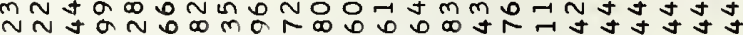

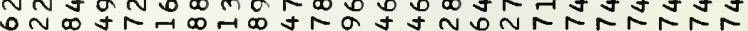
ON

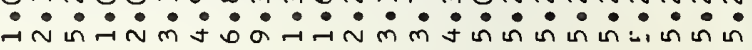

1न

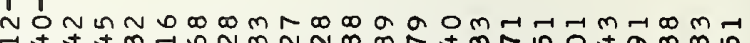

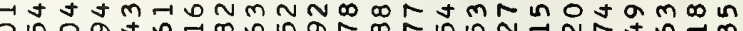

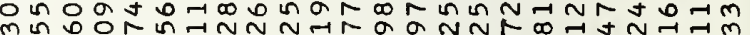
mڤn

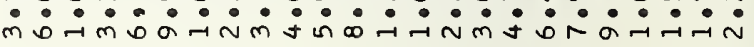

$m m \sim N \sim N-1-1-1$

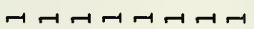

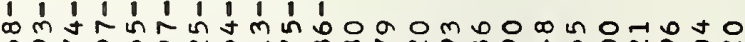
mañna

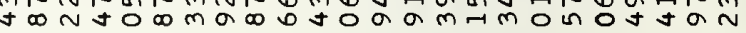

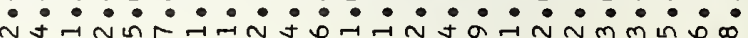
N ન

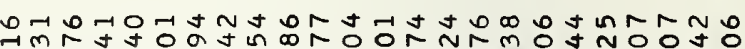
tmotato

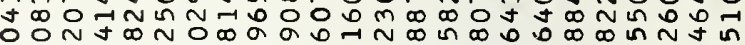

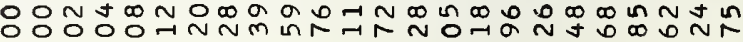
$\dot{m} \dot{m} \dot{m} \dot{m} \dot{m} \dot{m} \dot{m} \dot{j} \dot{j} \dot{0} \dot{0} \dot{-} \dot{-}-\dot{i} \dot{m} \dot{m}$

o n m n t $m m m N N N-1-1$

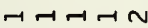

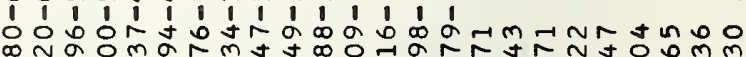

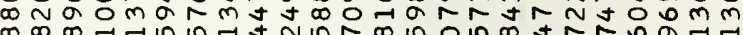

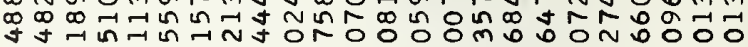

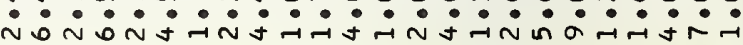

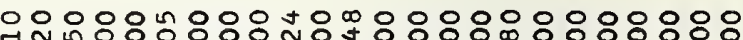

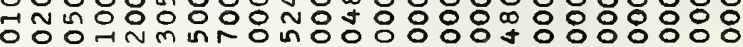

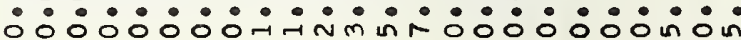

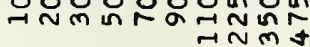

000000000000000000000000

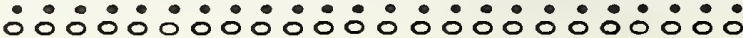
$\mathrm{mm} \mathrm{mm} \mathrm{mm} m \mathrm{~mm} m \mathrm{~mm} m \mathrm{~mm} \mathrm{~mm}$

a a a a a a a a a a a a a a a a a a a a

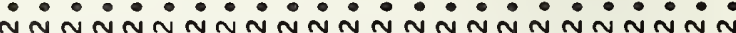

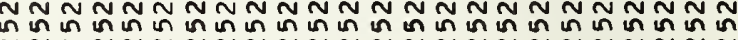

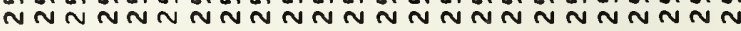




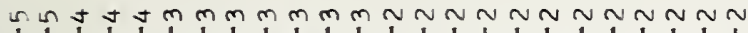
$\ll$

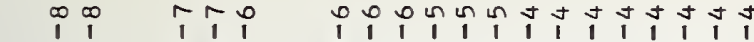

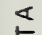

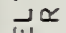
แே

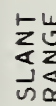

응

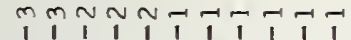

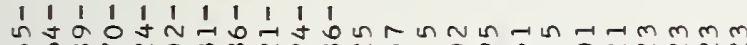

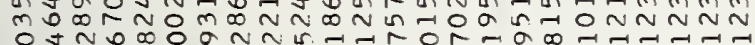
워 arat

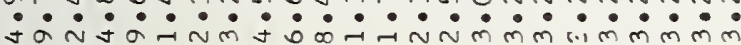

$\overrightarrow{1} \overrightarrow{1} \overrightarrow{1}$ $\neg-\sqcap-\neg-\neg N \sim \sim N \sim N \sim m m m$ mnthorntwo on $0 \rightarrow 1$
0,4

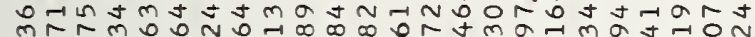
m

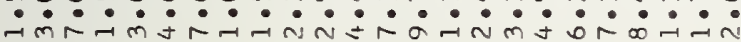

\&

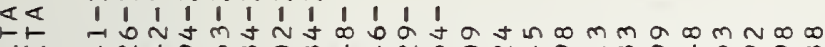

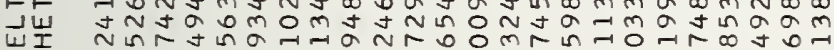

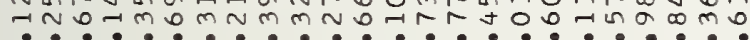

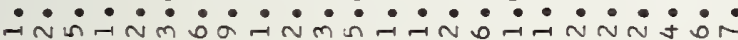

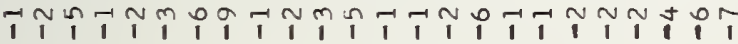

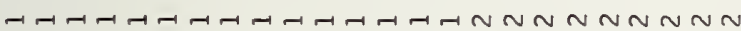

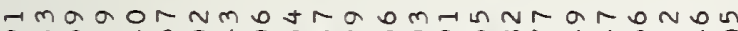

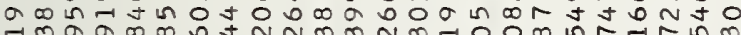

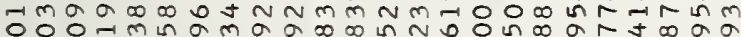

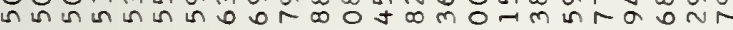
$\dot{0} \dot{0} \dot{0} \dot{0} \dot{0} \dot{0} \dot{0} \dot{0} \dot{\sim} \dot{\sim} \dot{\sim} \dot{\sim} \dot{\sim} \dot{\sim} \dot{\sim} \dot{\sim} \dot{m}$

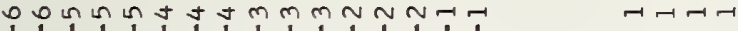

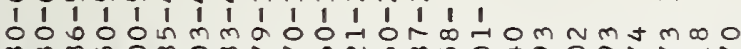
$\varangle$

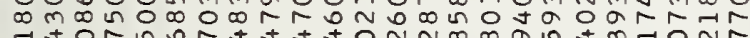

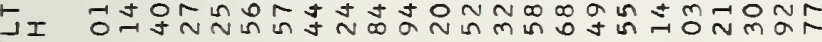
U.

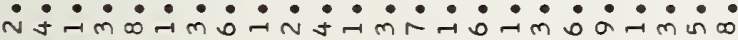

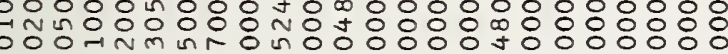

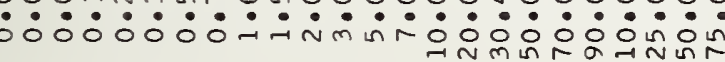

000000000000000000000000

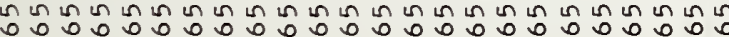

n

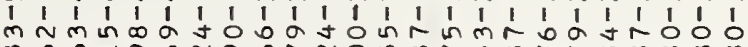

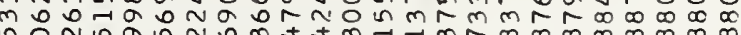
?

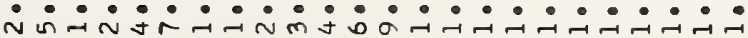

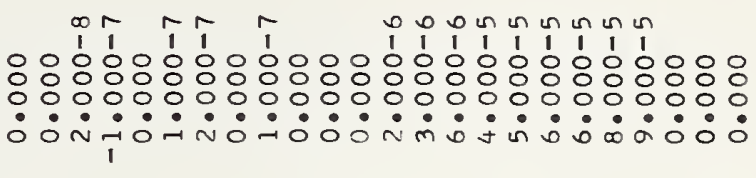
$\overrightarrow{1} \overrightarrow{1}$ I $\rightarrow a 0 n+b$ a

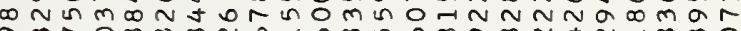

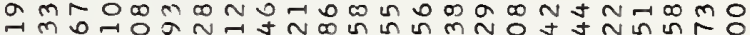

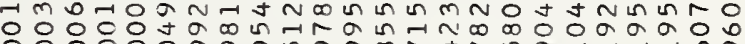

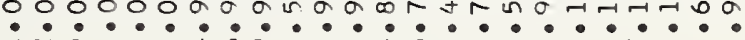

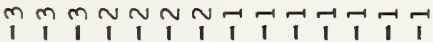

bractran

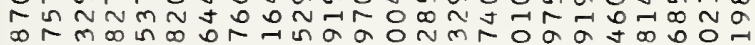
m

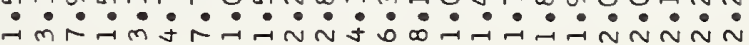

$m m \sim \sim \sim \sim-1+1+1+1$

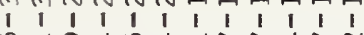

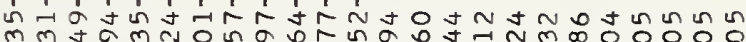
a m ina $\begin{array}{ll}m \\ m\end{array}$

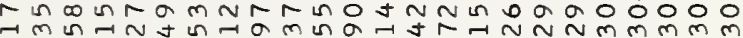
Hm $n$ ก $\dot{m} \dot{0} \dot{i} \dot{0} \dot{a} \dot{i} \dot{\sim} \dot{j} \dot{i} \dot{\sim} \dot{-} \dot{i} \dot{\sim} \dot{\sim} \dot{\sim} \dot{\sim} \dot{\sim} \dot{\sim} \dot{\sim}$

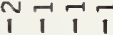

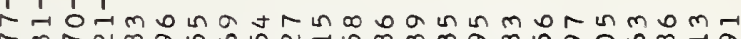
a m $m$ r

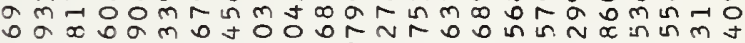

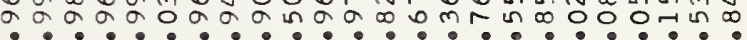

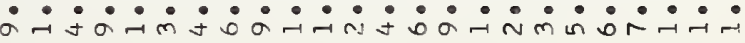

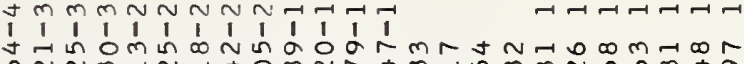

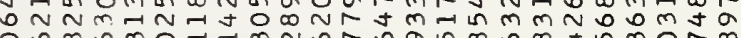

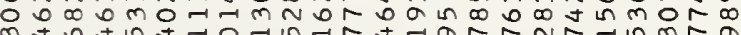

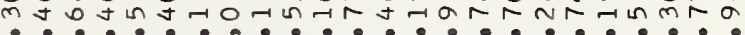

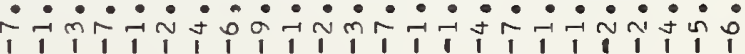
$\sim \sim N N \sim N N \sim N N \sim N N \sim N \sim N N N \sim N \sim \sim N$ N

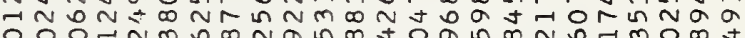

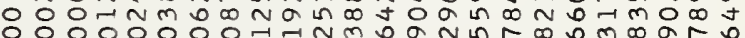
$00000000000000 \pi n m i n$

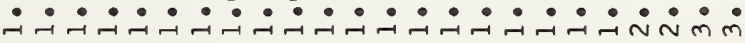

0 on

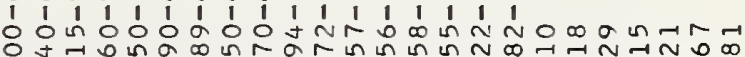

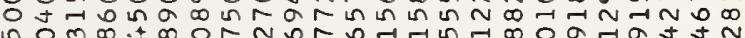
m

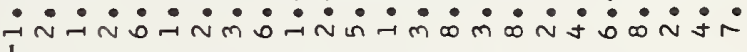

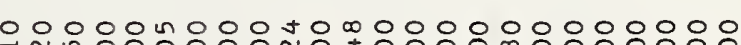

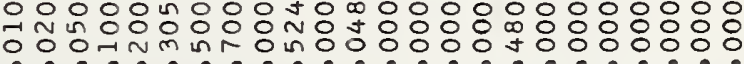

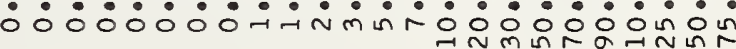
$\rightarrow \sim m g$

000000000000000000000000

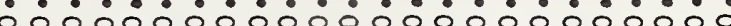
응응ㅇㅇㅇㅇㅇㅇㅇㅇㅇㅇㅇㅇㅇㅇㅇㅇㅇㅇㅇㅇㅇㅇㅇㅇㅇㅇㅇㅇㅇㅇㅇ ન્નાન

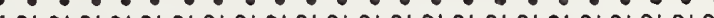
N N N N N N N N N N N N N N N N N N N N N N N

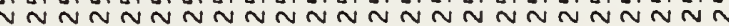




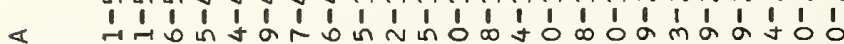

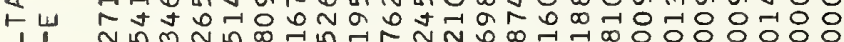

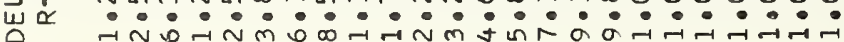

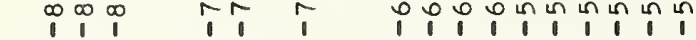

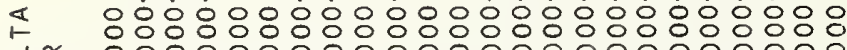
I $\alpha$ o 00000000000000000000000

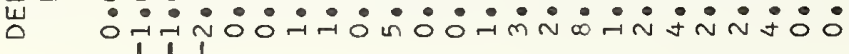
NTH-1

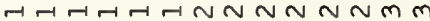

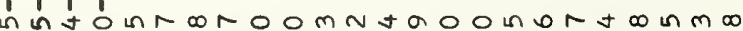

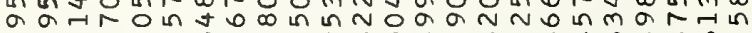
เய

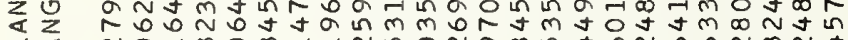

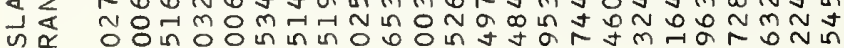

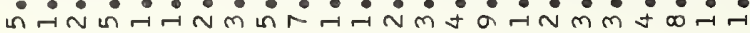

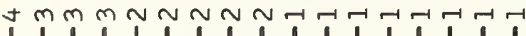

t $\infty N$ t n 范 म 品

w $R$ -

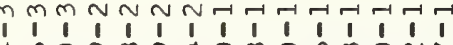

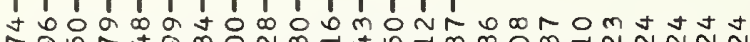

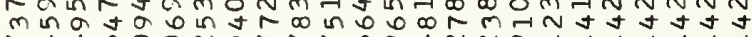

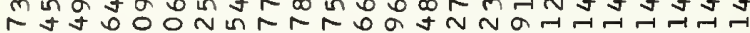

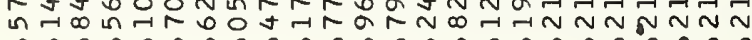

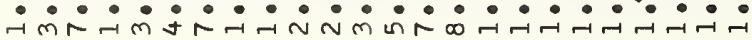
N $N-1+1$

U

$\sum^{2}$

is

ڤ

$\leftarrow \leftarrow$

岕岌

- $100 N+0 m m+0 N N+t \infty+a m m \circ \ln \pi$

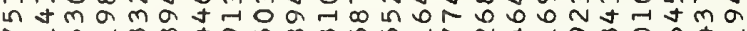
N

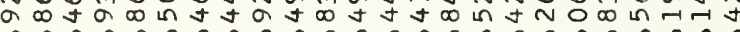

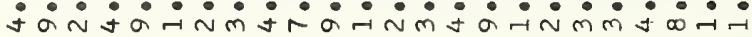

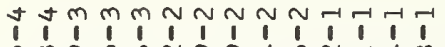

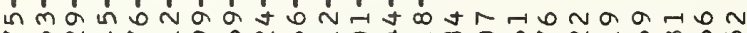

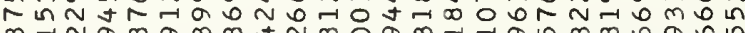

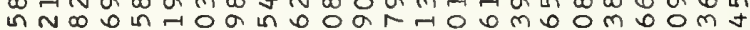

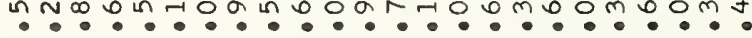

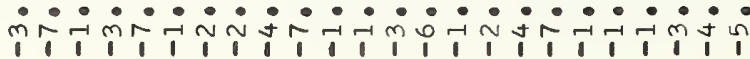
N N N N N N N N N N N N N N N N N

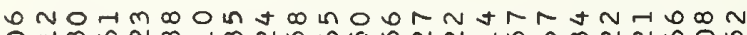

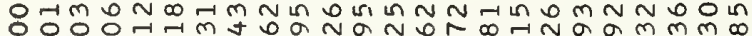

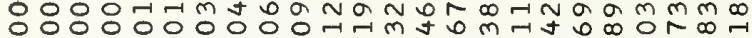

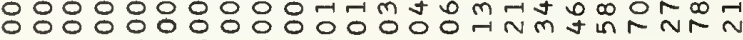
$\dot{N} \dot{N} \dot{N} \dot{N} \dot{N} \dot{N} \dot{N} \dot{N} \dot{N} \dot{N} \dot{N} \dot{N} \dot{N} \dot{N} \dot{m} \dot{j}$

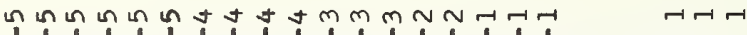

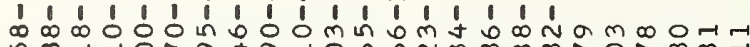

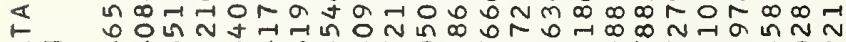
II

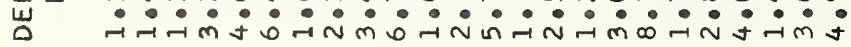

$\frac{T}{5}$

$\stackrel{5}{\frac{5}{5}}$

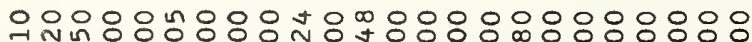

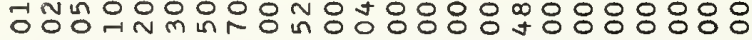

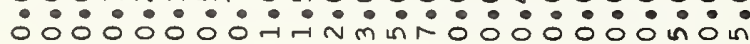
I

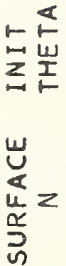

000000000000000000000000

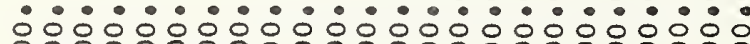
N N N N N N N N N N N N N N N N N N N N N

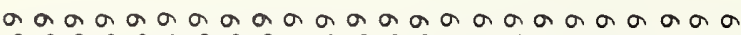

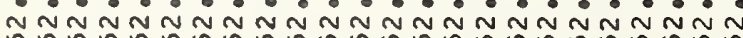

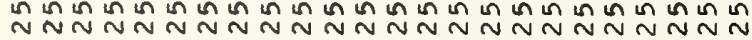

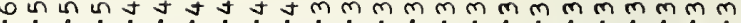
$\begin{array}{llllllllllllllllllllllll}1 & 1 & 1 & 1 & 1 & 1 & 1 & 1 & 1 & 1 & 1 & 1 & 1 & 1 & 1 & 1 & 1 & 1 & 1 & 1 & 1 & 1 & 1 & 1\end{array}$

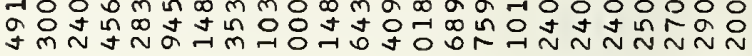

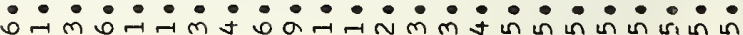

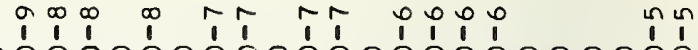

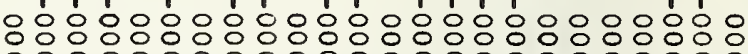

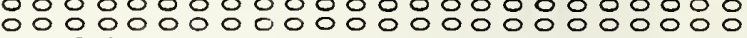

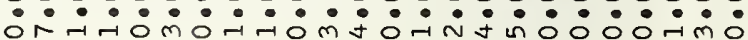

$10-17-1$

- $-4-\pi N N$ N N N M

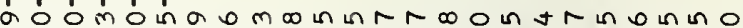

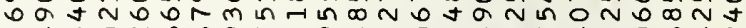
mamonobartatmontmrtnobata

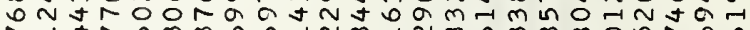

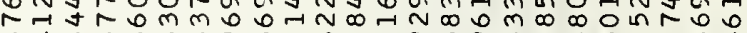
ป $\bullet \bullet \bullet \bullet \bullet \bullet \bullet \bullet \bullet \bullet \bullet \bullet \bullet \bullet \bullet \bullet$

tงmm

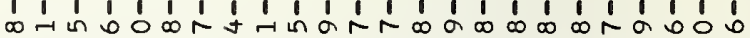
ōa

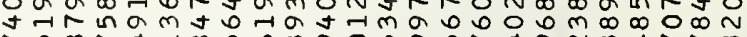

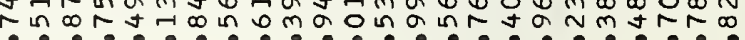

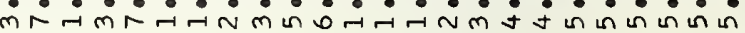

งmmmN N N N $\begin{array}{llllllllllllllllllllllll}1 & 1 & 1 & 1 & 1 & 1 & 1 & 1 & 1 & 1 & 1 & 1 & 1 & 1 & 1 & 1 & 1 & 1 & 1 & 1 & 1 & 1 & 1 & 1 \\ \infty & \infty & \alpha & 0 & 0 & 0 & m & n & \alpha & \sim & \alpha & m & 0 & \infty & v & \pi & -1 & \infty & N & 0 & m & m & m & m\end{array}$

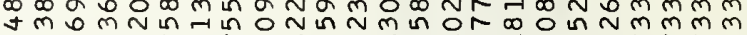

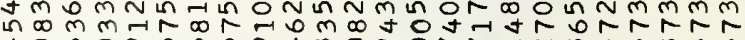
ป in

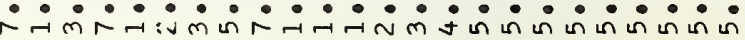

NNTH-TH

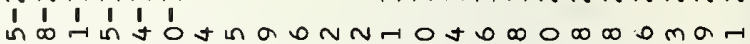
๙2

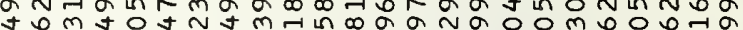

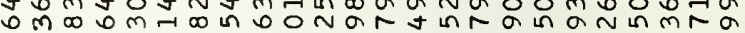

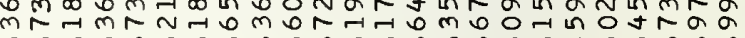

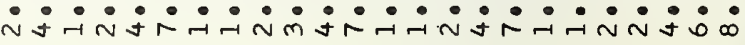

A t กำ बन NorbRry

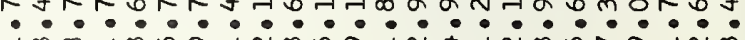

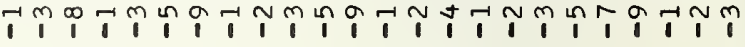

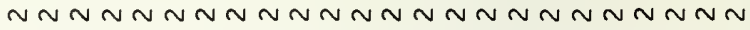

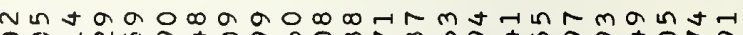

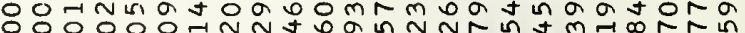

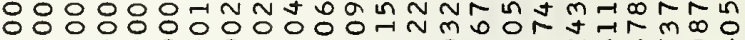

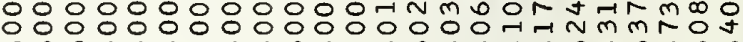

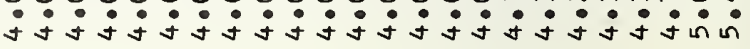

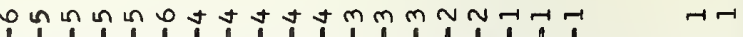
列 w t 0 O O in that

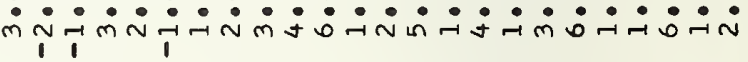

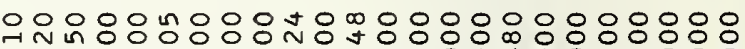

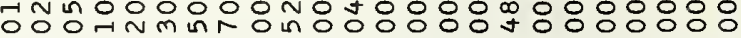

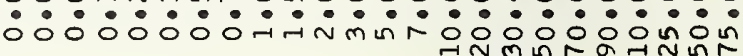

000000000000000000000000

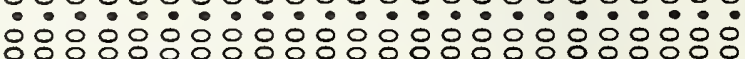

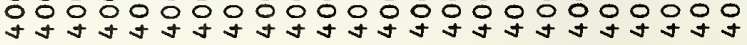

a a a a a a a a a a a a a a a a a a a a

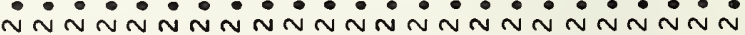

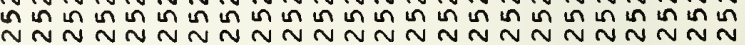


000000000000000000 in in

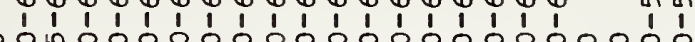

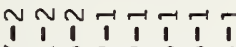

选茨

MN

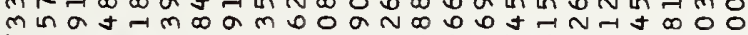
TNm⿻-1m-1 OnmR m m

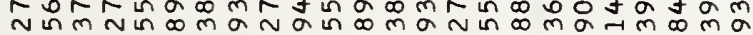

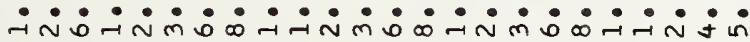

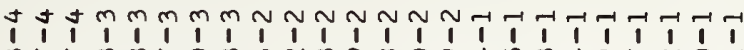
딴 mrRm

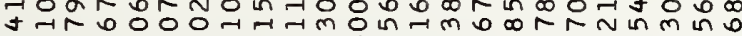
N In $N$ แn

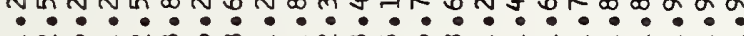

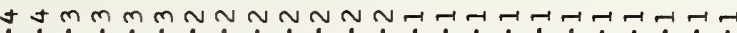

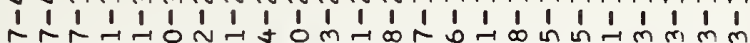
in mon

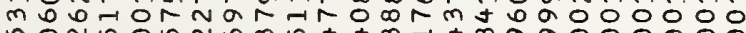

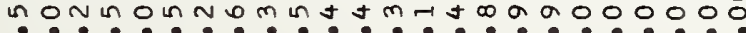

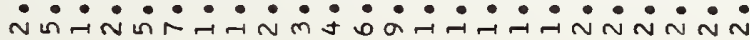

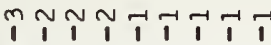

$\rightarrow-\rightarrow H \rightarrow H N \sim$

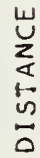

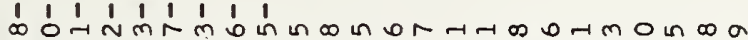
o on

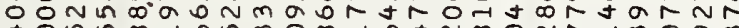

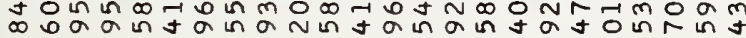

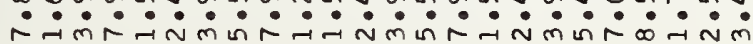

\&ष

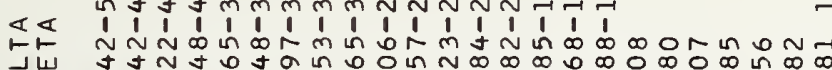
wI J ง

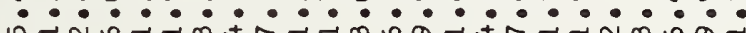
जि NNN N N N N N N N N N N N N N N N

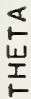

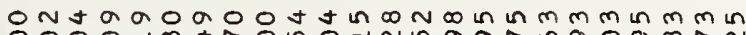

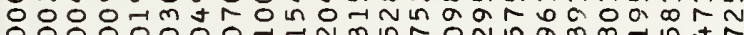

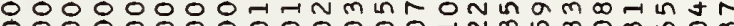
$0000000000000 \pi N m i n \infty m w 0 m$ $00000000000000000007-1$.

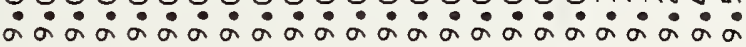

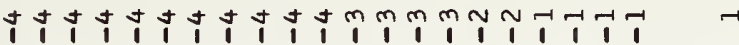
$+a r m-\infty-10000001-1000$

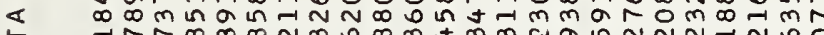
II N $\vec{u}^{I}$

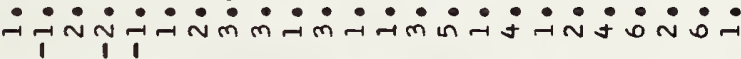

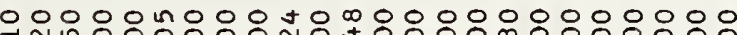
$\frac{5}{\frac{I}{I}}$

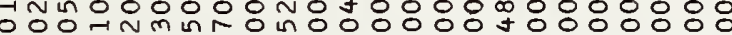

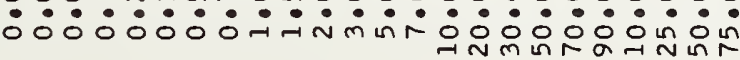

ᄂ

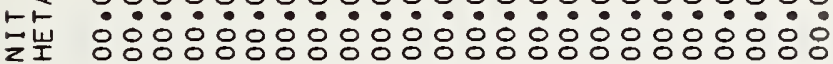

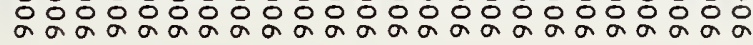


1111

n r O O O

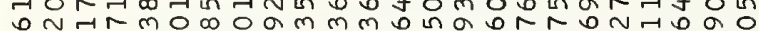
moromina a

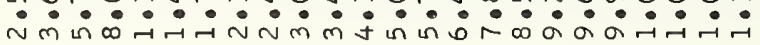

17 tarm $\infty$ o a tr. m m N

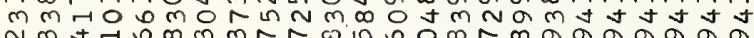

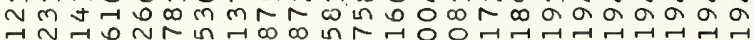

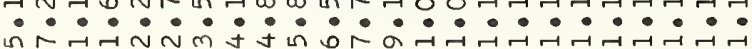

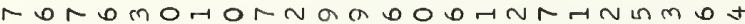

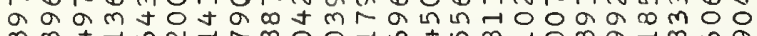

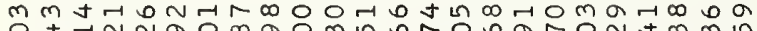

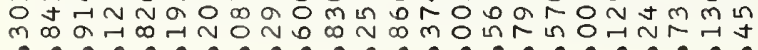

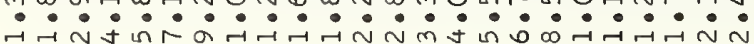

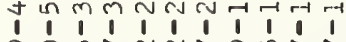

$\stackrel{1}{1}$

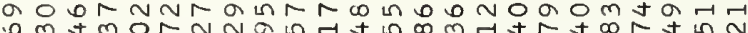
m t m ONNNGintin arr 10

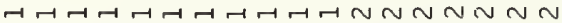

$\stackrel{\leftarrow}{\leftarrow}$

MOm

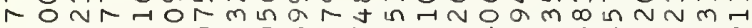

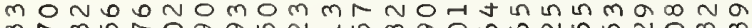

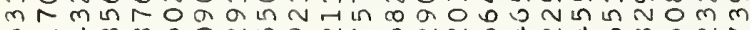

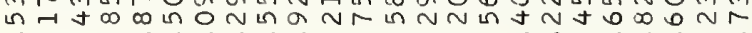
-

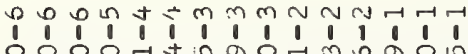

$\sqcap \sqcap \sqcap \neg \neg \sim$

$<$

I

0 in

$\underset{\omega}{\omega} I$

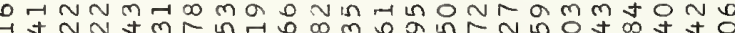

? ? ? ? ? ? ? ?

11

00000 n 000 t 0 mo00000000000

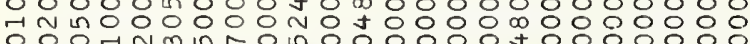

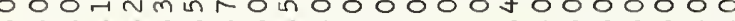

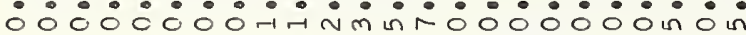
hom $\mathrm{m}$ in

000000000000000000000000 ல

Z

us

000000000000000000000000

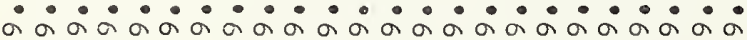

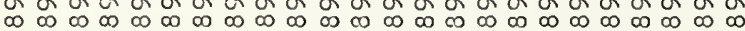

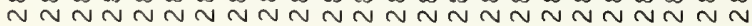

$m m m \sim N N M N N N N$ N

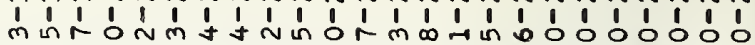

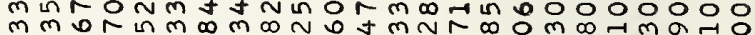
NNO Un a

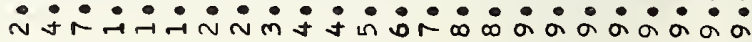

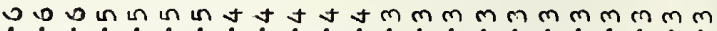
$\begin{array}{llllllllllllllllllllllll}0 & 0 & 1 & 1 & 1 & 1 & 1 & 1 & 1 & 1 & 1 & 1 & 1 & 1 & 1 & 1 & 1 & 1 & 1 & 1 & 1 & 1 & 1 & 1\end{array}$

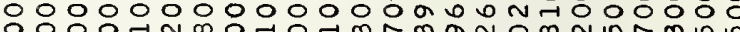

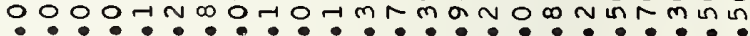

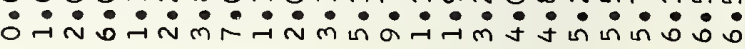

$\neg-H-H-N N N N N N N N N$ N O $m$ OHAONON 0 in

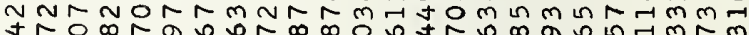

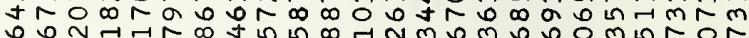
ง

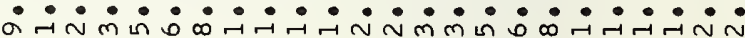

$\dddot{1}$

$\neg \sqcap-$

a onr $\begin{gathered}1 \\ 0\end{gathered}$

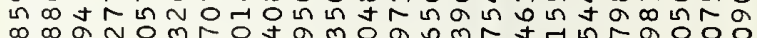

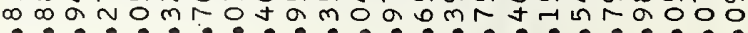

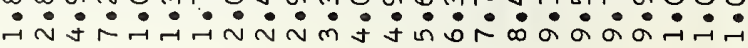

II

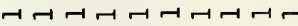
R

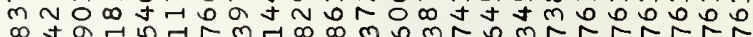

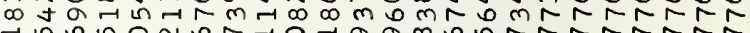

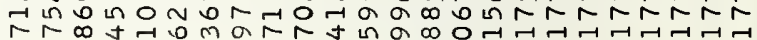

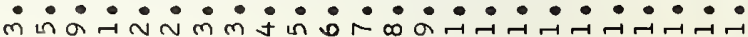

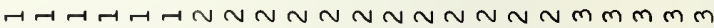
Int N N N $N$ I N N O I T 4 $0 N-1 N \infty$ J

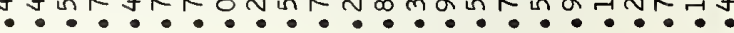

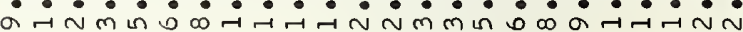

it

નҒનҒનҒનҒન

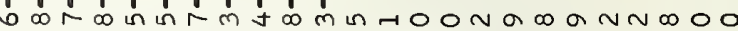

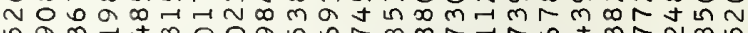

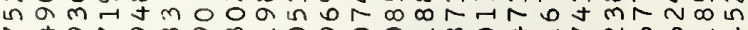

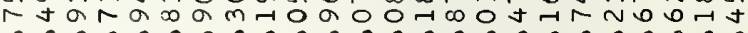

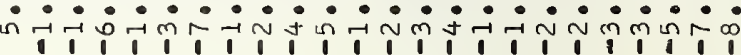

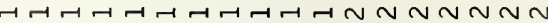

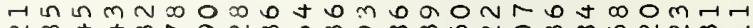
$N \infty$ t

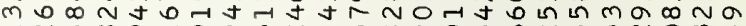

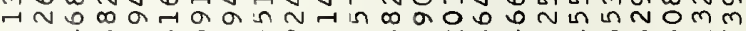

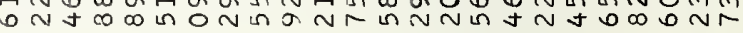

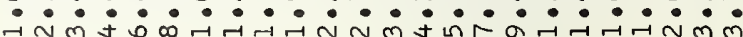

D.

$\sqcap \sqcap \sqcap-\sqcap \curvearrowright$

a in $\mathrm{m}$ in $\rightarrow \mathrm{m}$ $\rightarrow$ in

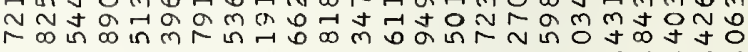
11

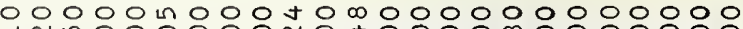

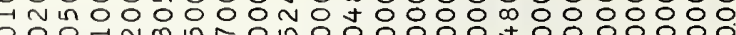

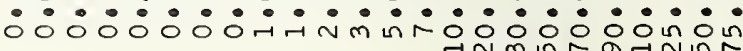
rNm

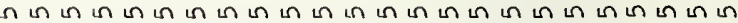
ல்

000000000000000000000000

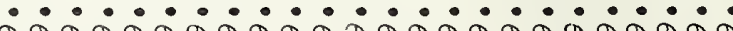
舟

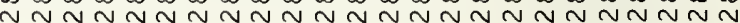




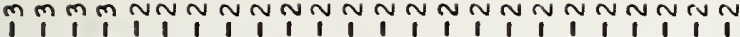
« J 11 o u

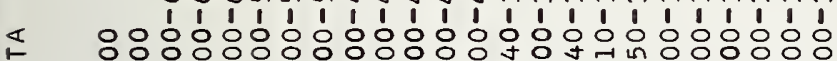

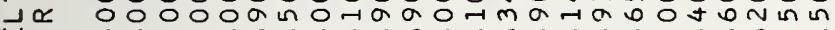
ш

-

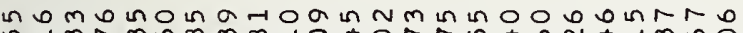

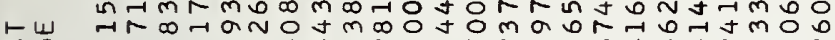

$\geq 0$ Trm

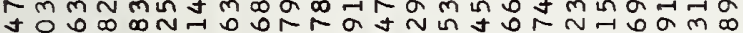
†० 옹ำ

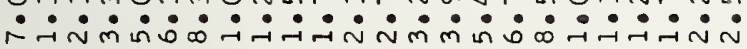

1િન

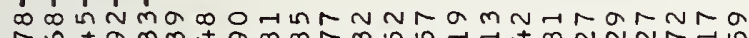

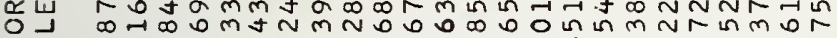

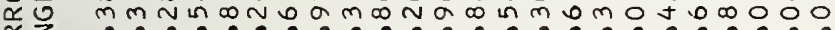

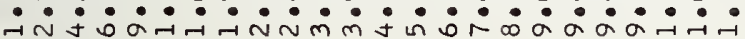

$\neg-1$

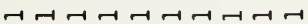

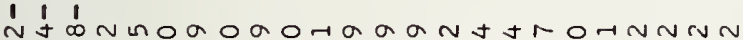

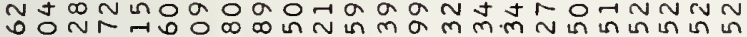

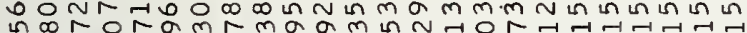

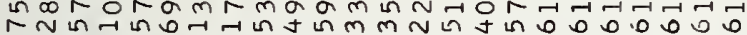

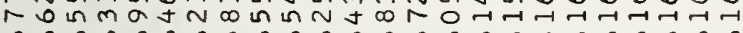

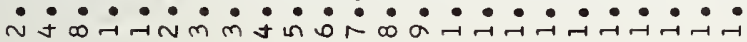

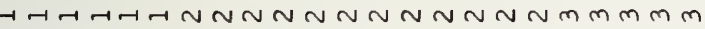

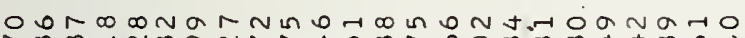
I

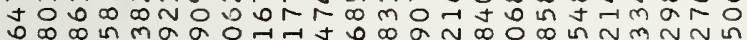

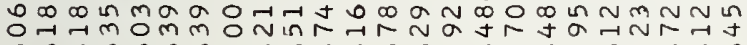

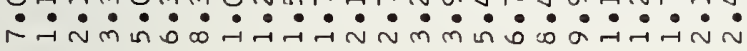

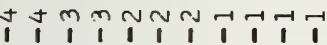

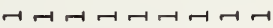

$\varangle \ll$

岗岌

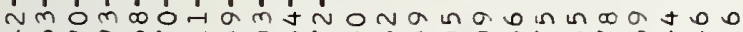

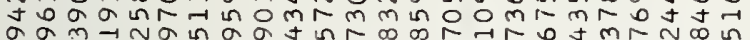

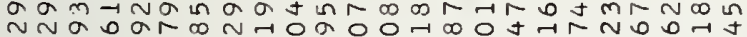

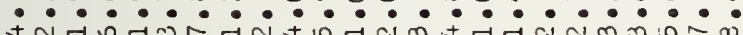

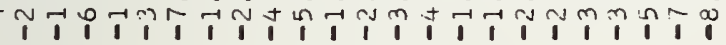

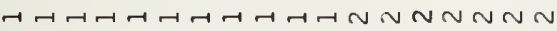
$\underset{⿱ ㇒}{\stackrel{I}{I}}$

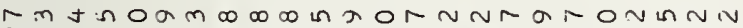
an $n$ o

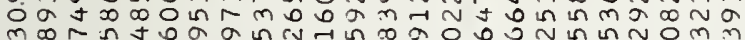

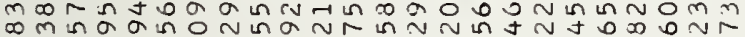

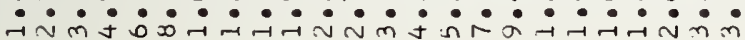

00 on 2 a

$\begin{array}{llllllllllllllll}1 & 1 & 1 & 1 & 1 & 1 & 1 & 1 & 1 & 1 & 1 & 1 & 1 & 1 & 1\end{array}$

$\neg \neg-\neg-N$

to

$\ll$

II

岃

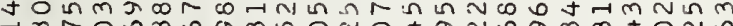

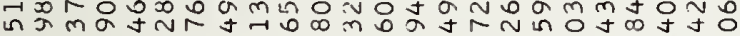

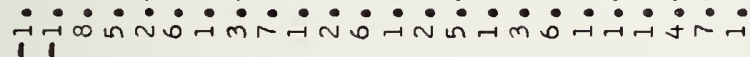

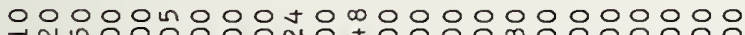

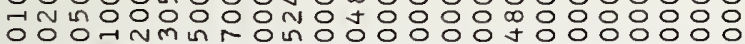

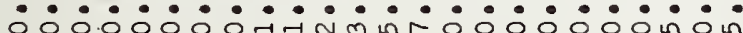

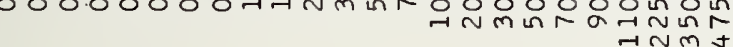

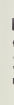

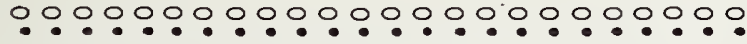

$\because \leftarrow$

¿

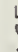

选

$\frac{u}{a} z$

000000000000000000000000

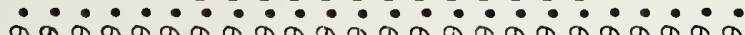

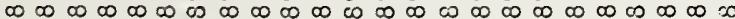

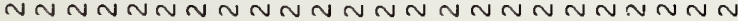

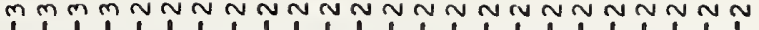

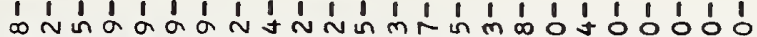
imñy

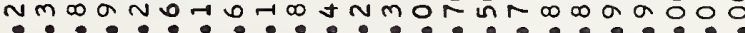

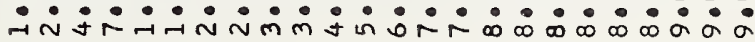

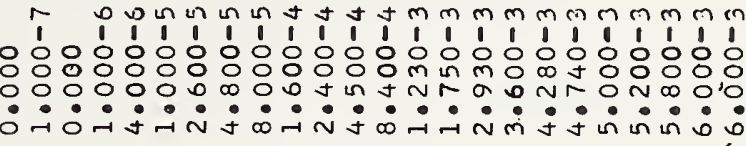

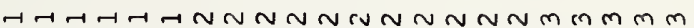

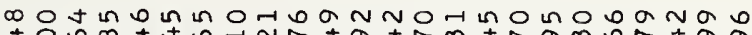

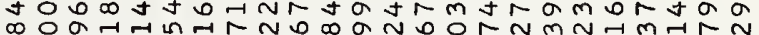
m윰ำ maj

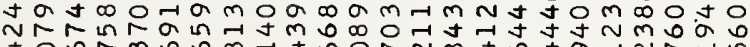

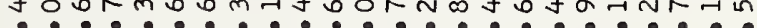

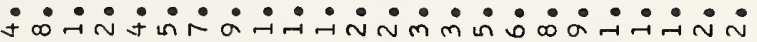

$\begin{array}{llll}1 & 1 & 1 & 1\end{array}$

m n'

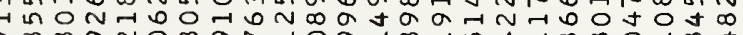

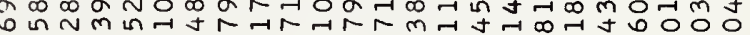

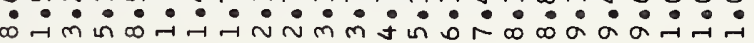

$\overrightarrow{1} \overrightarrow{1}$

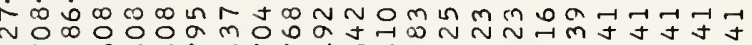

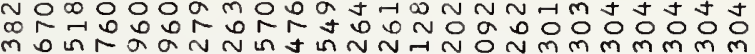
M

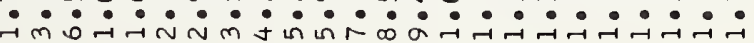

- $-1-\rightarrow N N$ N

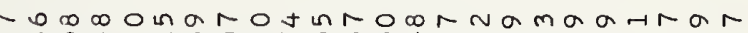

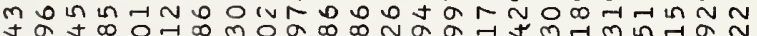
N ง

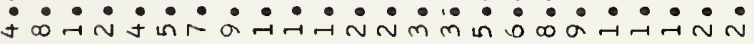

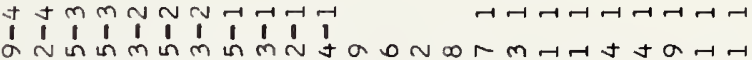

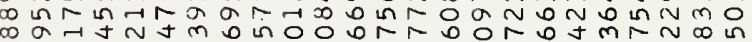

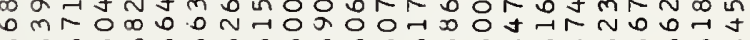
$\begin{array}{llllllllllllll}1 & 1\end{array}$

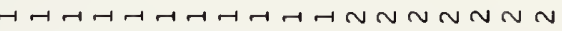

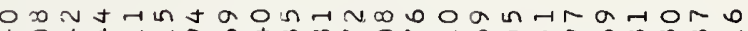

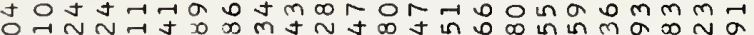

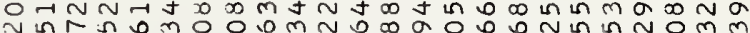
ก

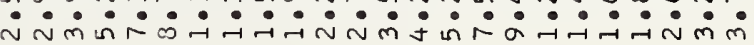

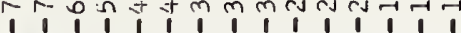

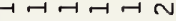

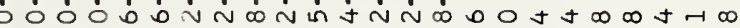

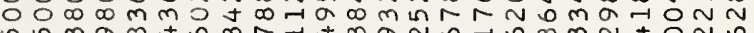

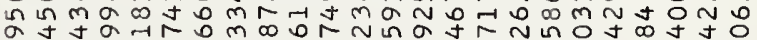

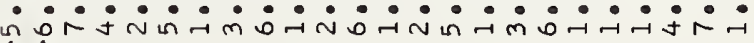
i

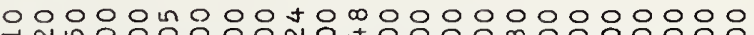
元

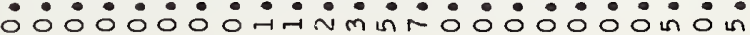
$\rightarrow \sim \underset{m}{\sim}$

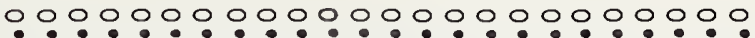
$\dot{N} \dot{N} \dot{N} \dot{N} \dot{N} \dot{N} \dot{N} \dot{N} \dot{N} \dot{N} \dot{N} \dot{N} \dot{N} \dot{N} \dot{N} \dot{N} \dot{N} \dot{N}$

000000000000000000000000

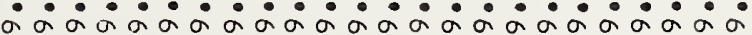

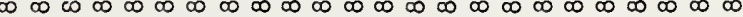

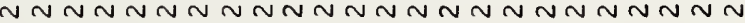


$\stackrel{4}{1}$

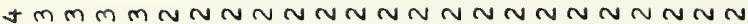

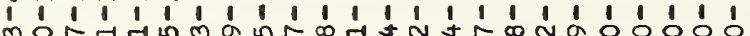
$r$ in 0 a $0 N$ in a m m n $N$ m n

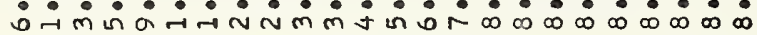

$\underset{\omega}{\stackrel{5}{\sigma}}$

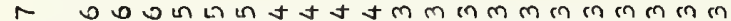

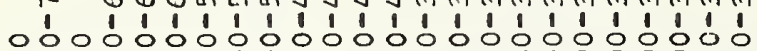

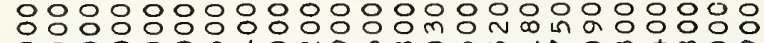

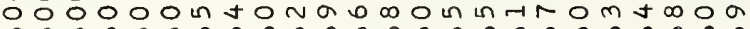

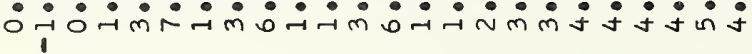

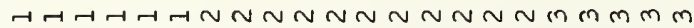

t $m$ o tamo in $\infty$ H t N N

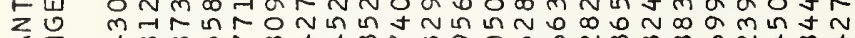

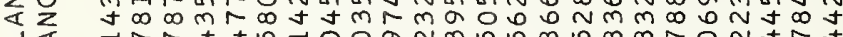

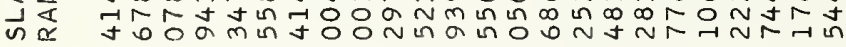

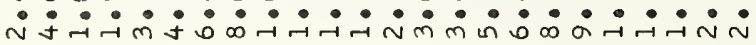

$N N-1-1-1$

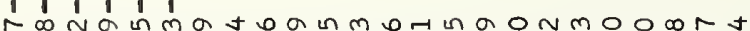

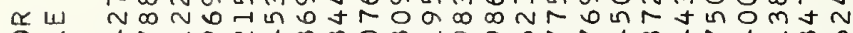

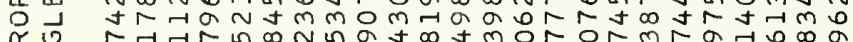

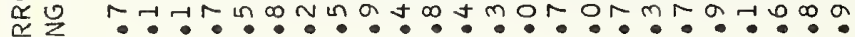
w 4

$-1-1-1-1-1-1$

1111

t

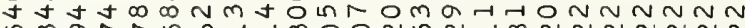
in markñ

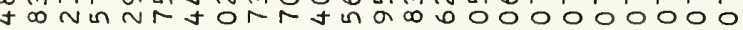

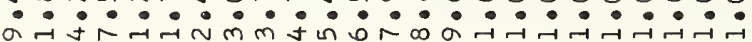

$\neg-1-1-N \sim N \sim \sim \sim N \sim \sim N \sim m m m m m$

U

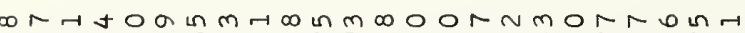
i t ง

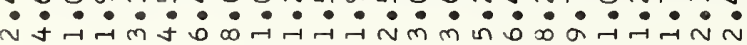

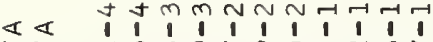

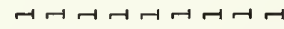

$\leftarrow$

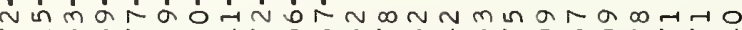

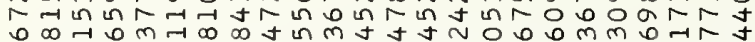

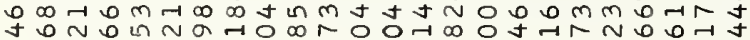

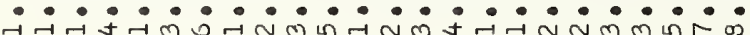
।

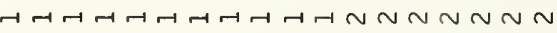

$\underset{\leftarrow}{\stackrel{\leftrightarrows}{I}}$

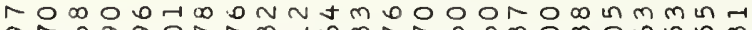

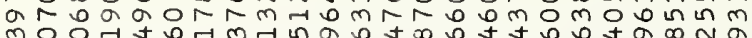

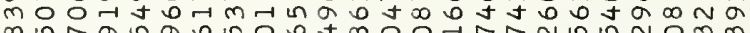

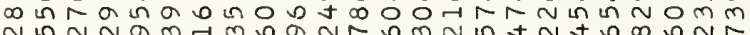

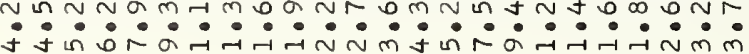

Trom

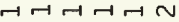

$\ll$

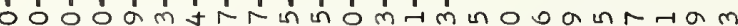
Oom o o o I 岁

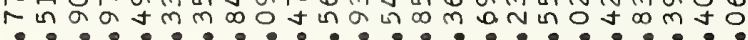

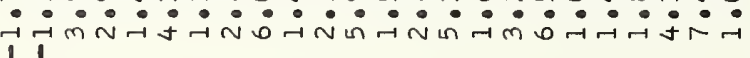

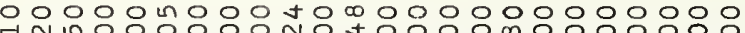

$\stackrel{1}{1}$

岌

R =

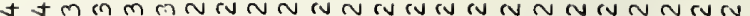
$\begin{array}{llllllllllllllllllllllll}1 & 1 & 1 & 1 & 1 & 1 & 1 & 1 & 1 & 1 & 1 & 1 & 1 & 1 & 1 & 1 & 1 & 1 & 1 & 1 & 1 & 1 & 1 & 1\end{array}$

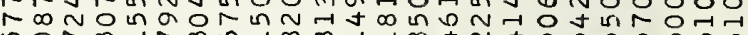

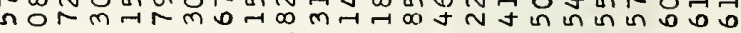

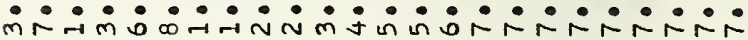

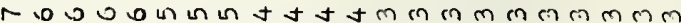

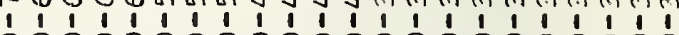

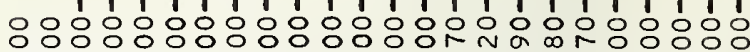

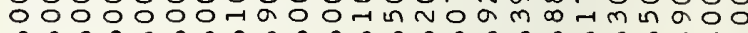

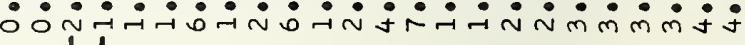
- $-1-r N$ N

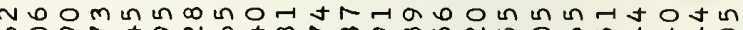
o oaryandatinam

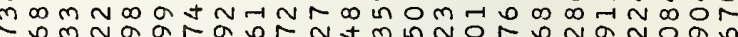

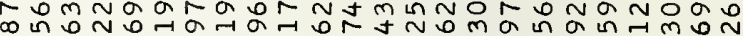

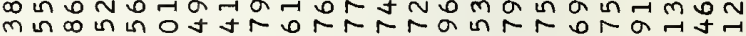
กง

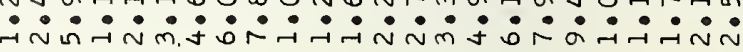

$\sin 1,9+1$

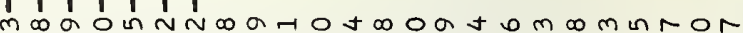

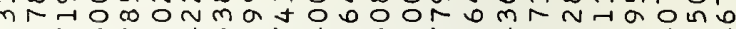

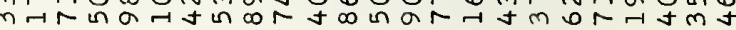
t $\infty \pi n-10 \sigma-t a m a \infty$ t

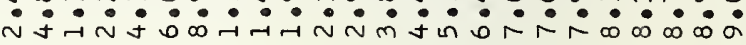

$\operatorname{sent-1} t$

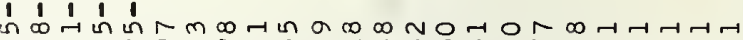

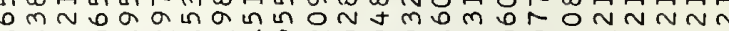

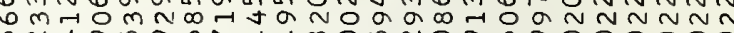
D N

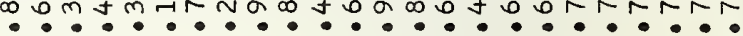

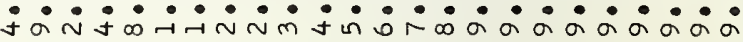

Hन- $\rightarrow$ a a on $n$ - 0 -1

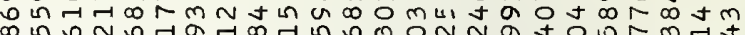
min

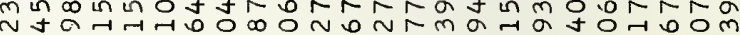

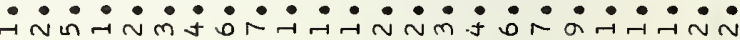

un

નન

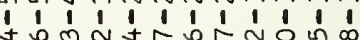

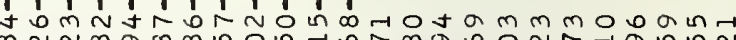
$\infty \sim N \infty a \infty \infty$ in 0 in

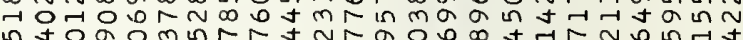
年 î -

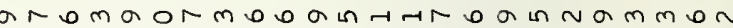

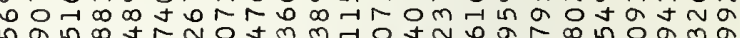

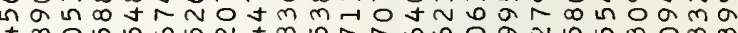

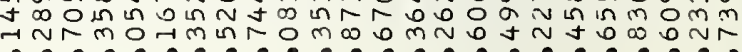

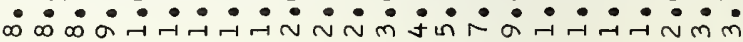

$\infty \infty \infty$ เn

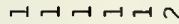

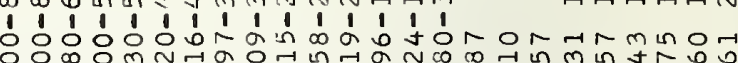
然

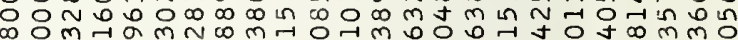

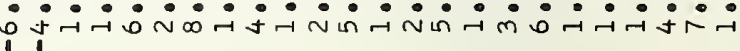

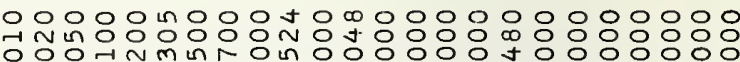

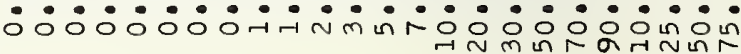
$\rightarrow \sim m s$

000000000000000000000000

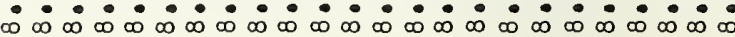

000000000000000000000000

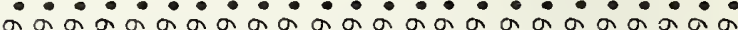

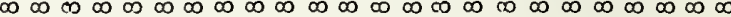
$N N N N N N N N N N N N N N N N N N N$ 


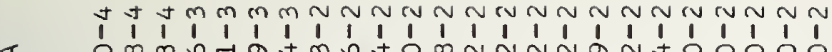

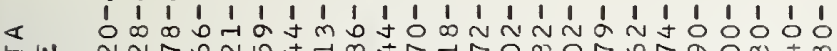
I

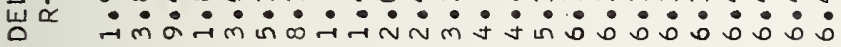

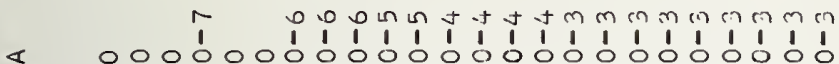

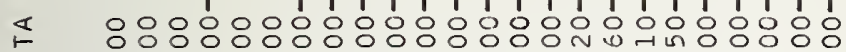

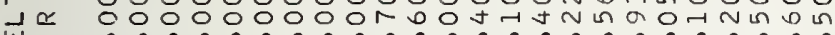

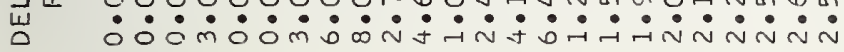

1.

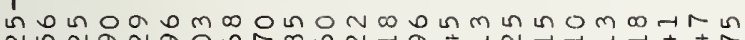

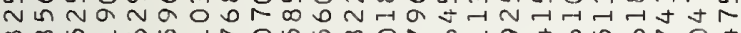
ŁU山

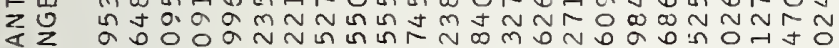

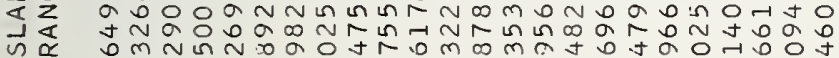

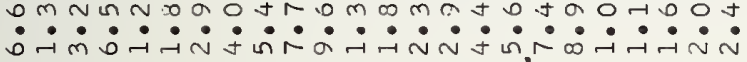

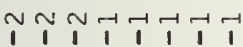

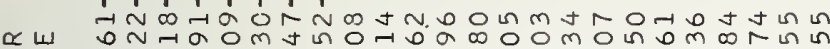

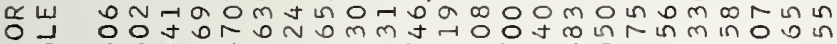
a jumant

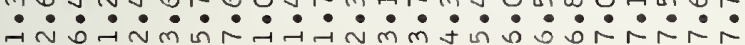

$\sim \sim \ln ^{1}+1-1+1$

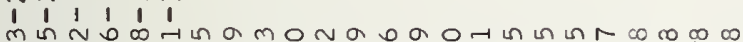

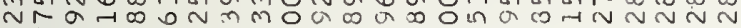

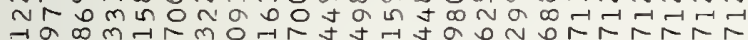

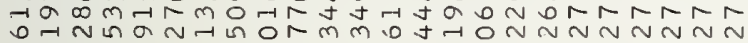

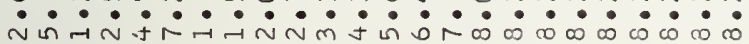
1.

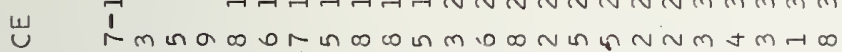

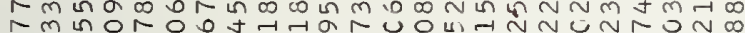

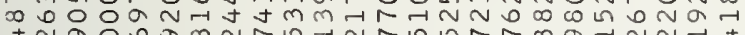

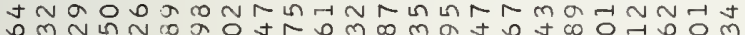

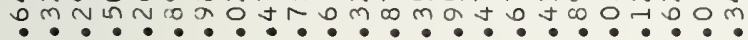

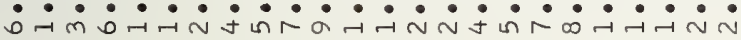

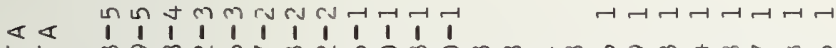

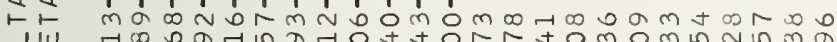
壵 崖 in Hન-

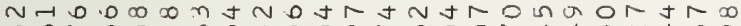
Dorborl r. O เก in in

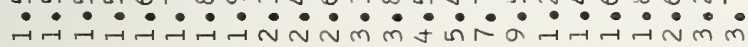

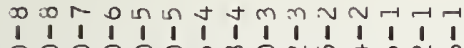

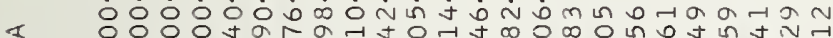
JI

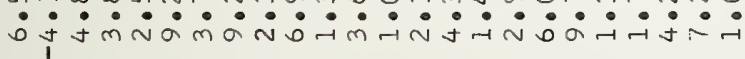

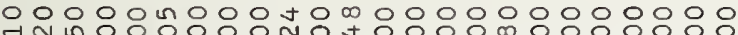

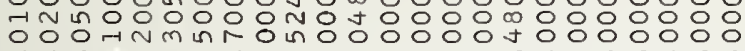

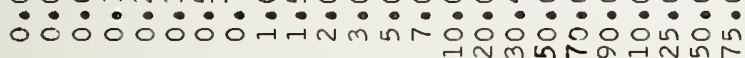

000000000000000000000000 ஸे in in

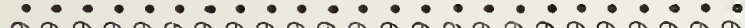

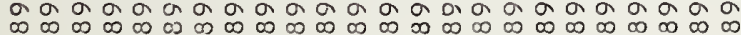

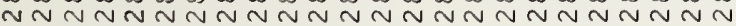

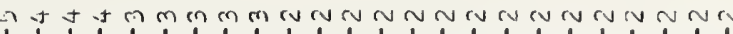

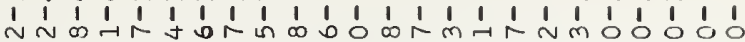

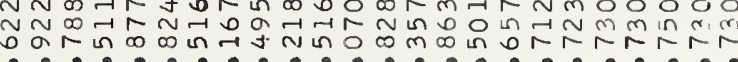

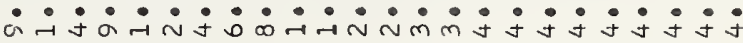

$\cos r$

111

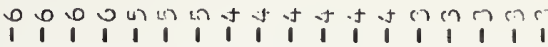

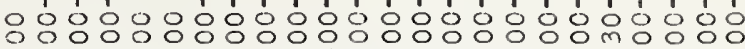

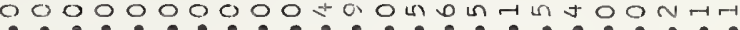

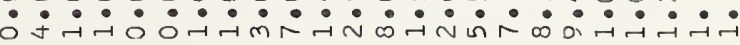

$\ddot{1}+1$

n⿺𠃊万丨

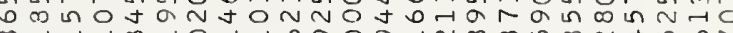

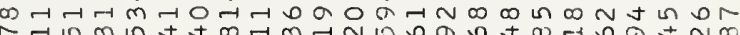
ન

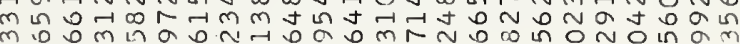

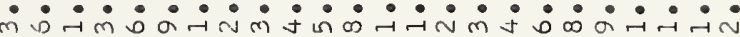

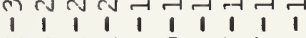

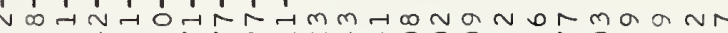

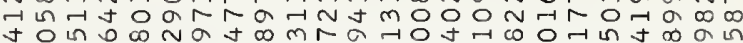

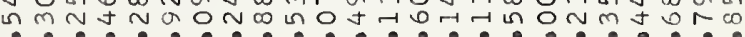

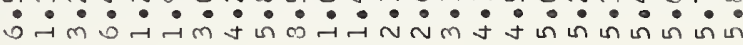

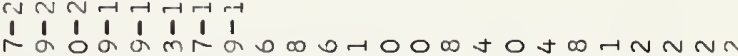

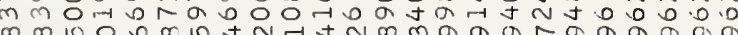

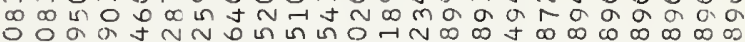

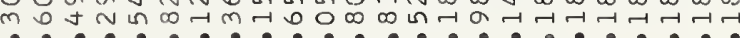

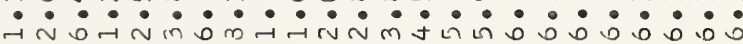

11

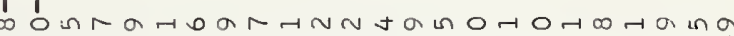

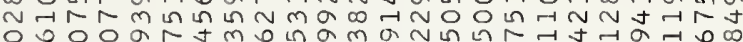

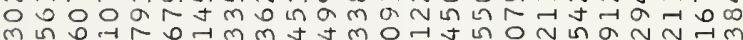

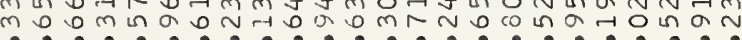

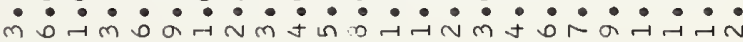

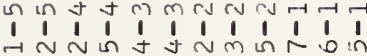

t Nom

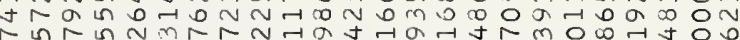
a ba ง

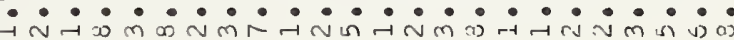

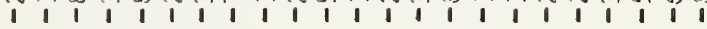

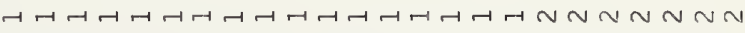

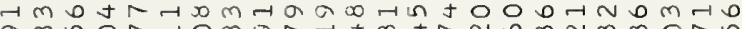
a

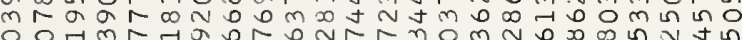

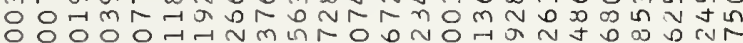

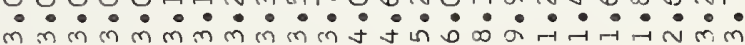

T-

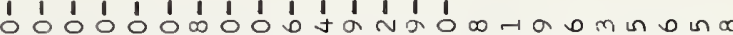

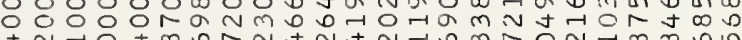

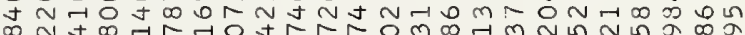

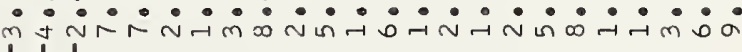
00000 m 000700000000000000

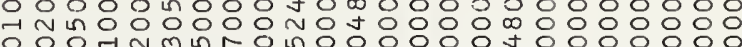
$000 n \mathrm{~m}$ Ln

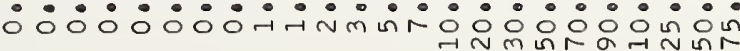
$\rightarrow \sim m s$

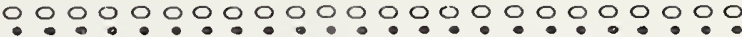

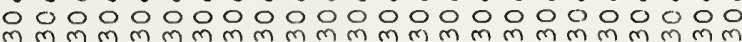

000000000000000000000000 - a a a a a a

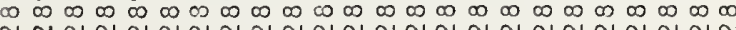




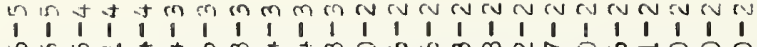

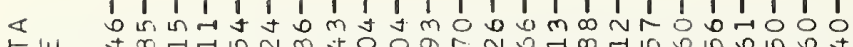

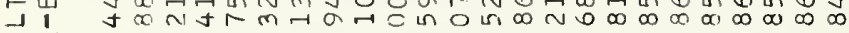

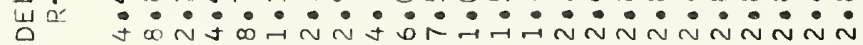

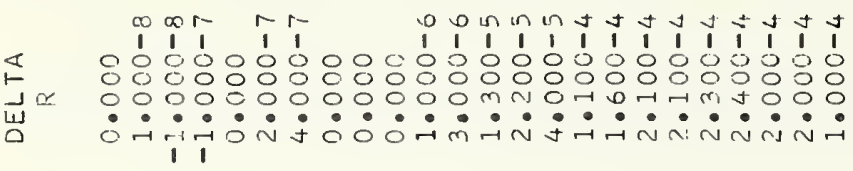
$\overrightarrow{1} \vec{i}$

$\stackrel{\text { ŁU }}{\sum}$ u) $\frac{1}{\alpha}$

onirrmogoa in a.m $m$ in $-\pi$ a $\alpha \sim a \sigma m \rightarrow m a t \rightarrow r \infty w a r m a m a 0 a-0 N$

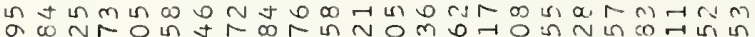
min

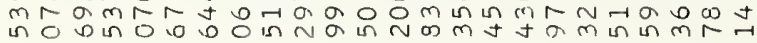

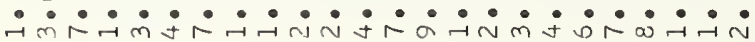

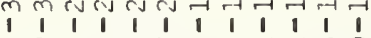

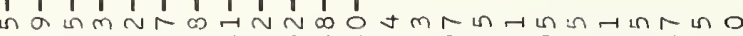

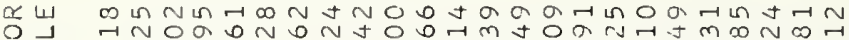

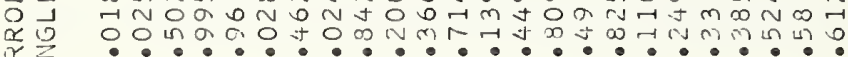

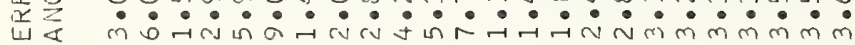

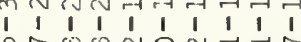

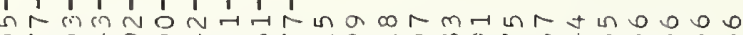

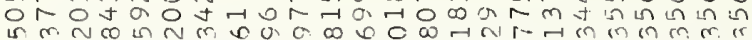

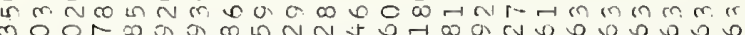

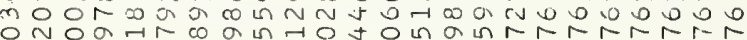

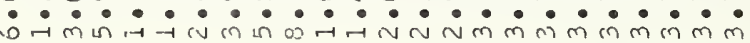

11

I NaND $m a n-a r a$ a ONT b

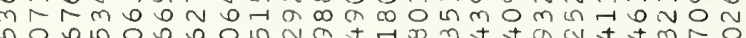
n?

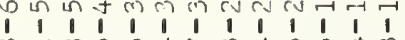

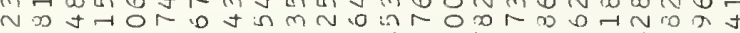

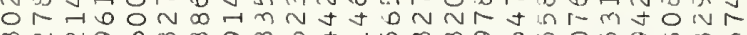

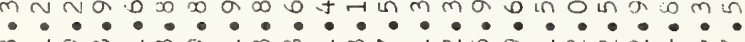

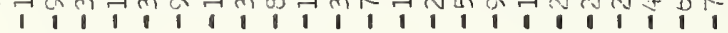

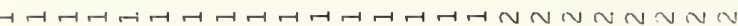

0 .

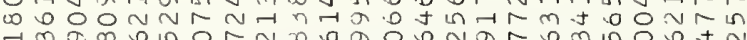

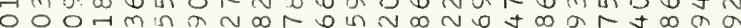
in

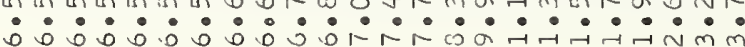

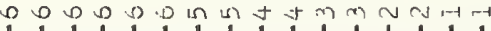

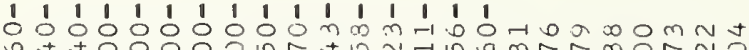

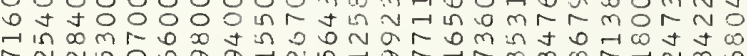

$+1$

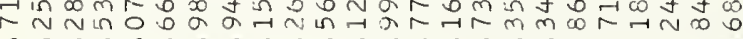

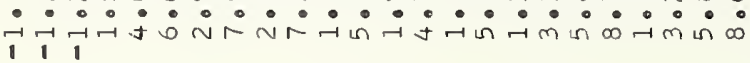

$00000 \mathrm{~m} 000$ a n 0000000000000

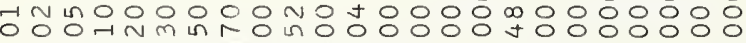

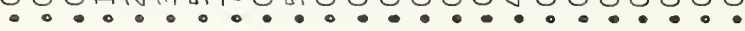

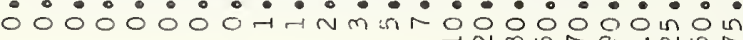
$\rightarrow$ m

ᄂะ

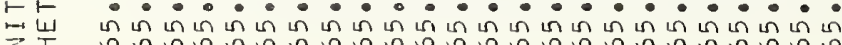
zI

000000000000000000000000

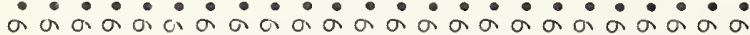

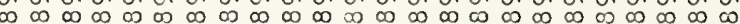
$N \sim N \sim N N N \sim N R N N R N N N N \sim N \sim N \sim N N$

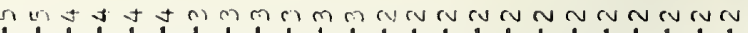

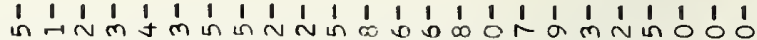
a

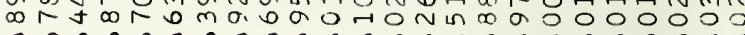

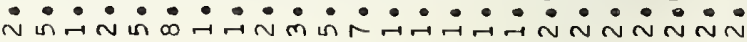

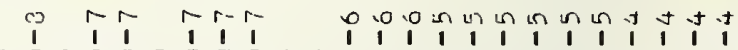

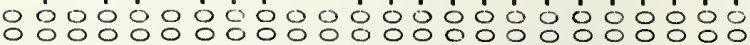

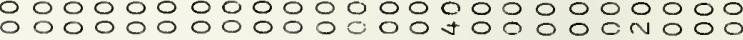

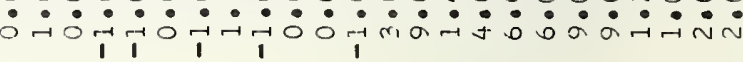

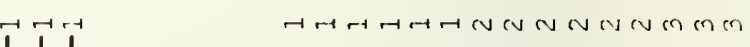

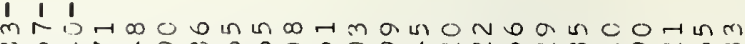

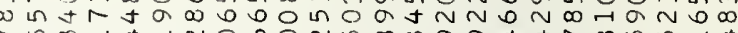

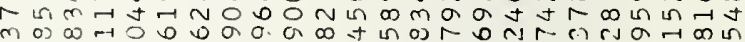

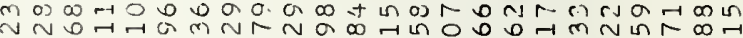

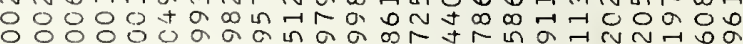

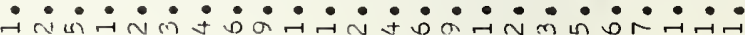

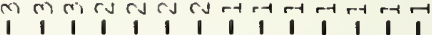

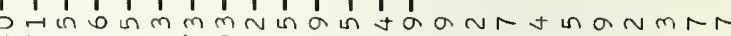

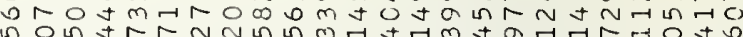

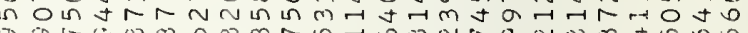

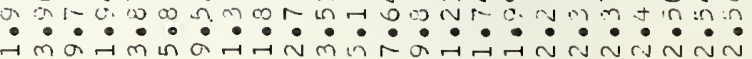

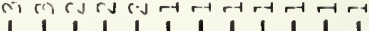

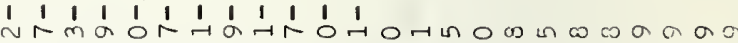

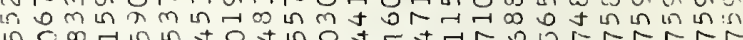
in 0 o $\begin{aligned} & -1 \\ & \text { in }\end{aligned}$ नَন

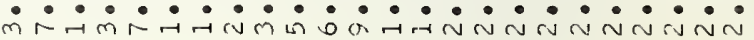

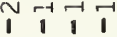

H F-

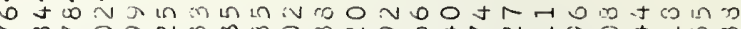

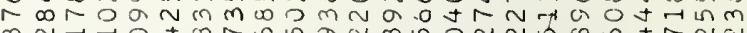
m N

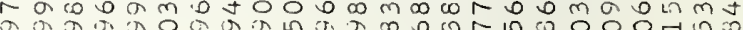

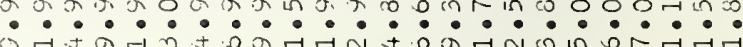

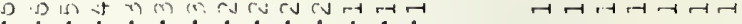

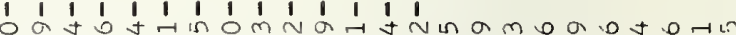

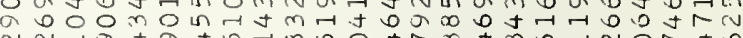

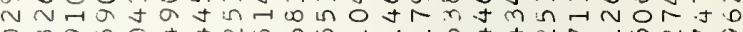

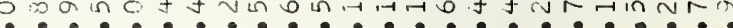

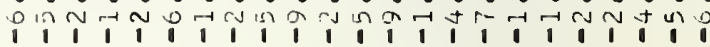

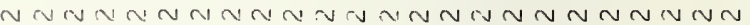
H Nin

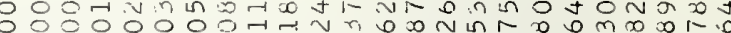

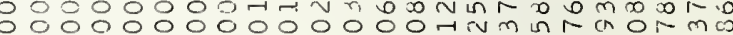

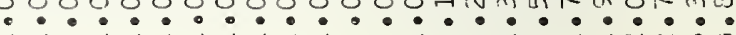

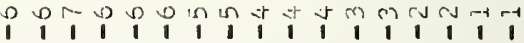
$\mapsto \mapsto-1$

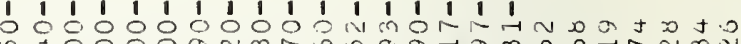

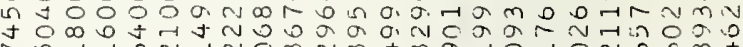

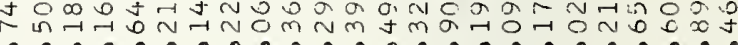

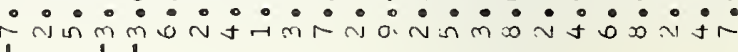

$00000 \mathrm{mon} 0 \mathrm{0}, 0 \mathrm{mo00000000000}$ 구의.

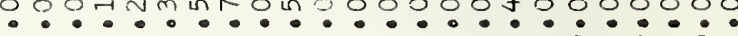
$\dot{0} \dot{0} \dot{0} \dot{0} \dot{0} \dot{0} \dot{1} \dot{0} \dot{0} \dot{0} \dot{0} \dot{0} \dot{0} \dot{0} \dot{0}$ $\rightarrow N m$ J

000000000000000000000000 $\dot{0} \dot{0} \dot{0} \dot{0} \dot{0} \dot{0} \dot{0} \dot{0} \dot{0} \dot{0} \dot{0} \dot{0} \dot{0} \dot{0} \dot{0} \dot{0} \dot{0} \dot{0} \dot{0} \dot{0}$

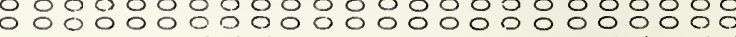

000000000000000000000000

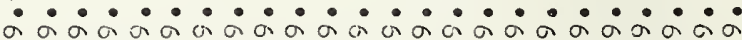

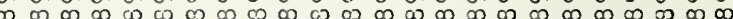
$\sim \sim N \sim N \sim N N N \sim N N N \sim N N \sim N N N \mathbb{N}$ 


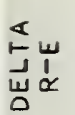

un -

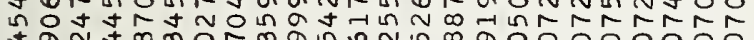
tont mormann

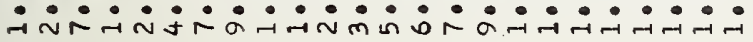

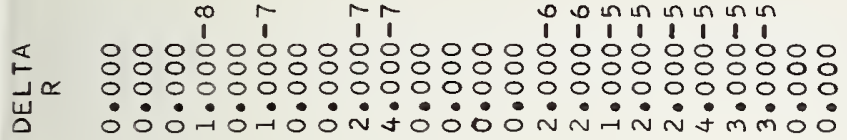

$\hat{1} \overrightarrow{1}+1$

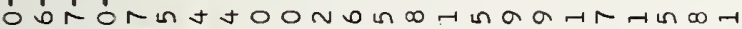
- 5 N in 上U

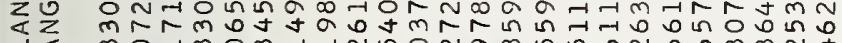
in $\frac{1}{\alpha}$

幽

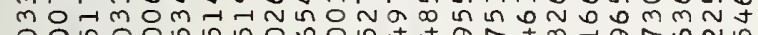

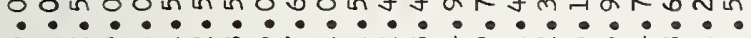

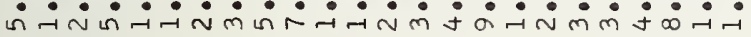

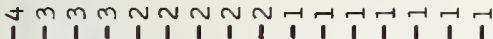

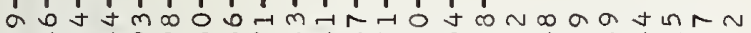

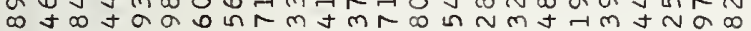

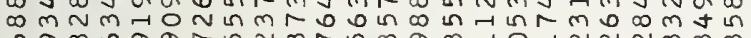
:

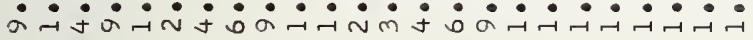

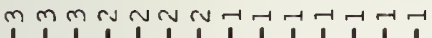

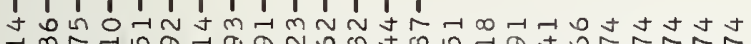

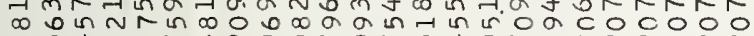

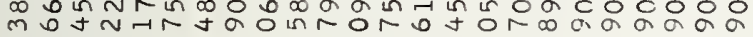
nळu

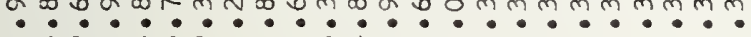

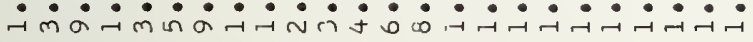

N N

$u$
$z$
$\frac{u}{z}$
$\frac{5}{0}$

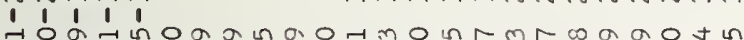

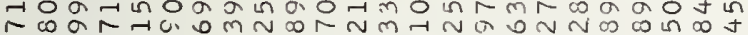
N

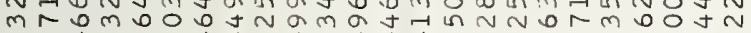

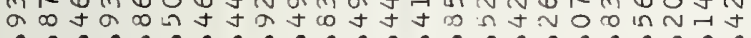

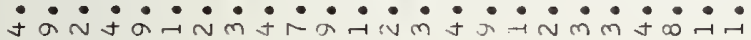

i.

$\varangle \varangle$

岁㞱

岁吉

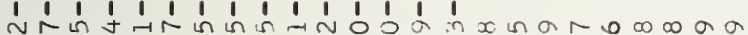

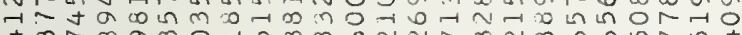

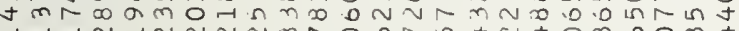

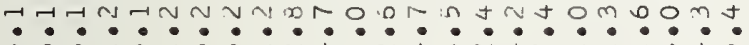

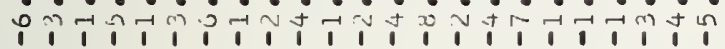

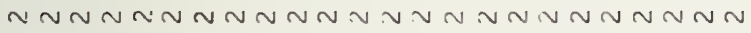
细

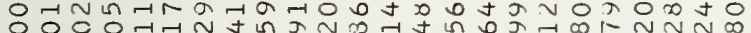

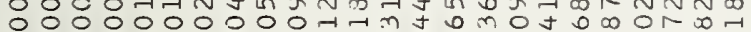

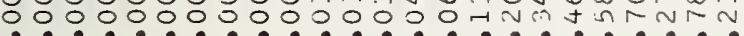
$\dot{\sim} \dot{\sim} \dot{\sim} \dot{\sim} \dot{\sim} \dot{\sim} \dot{\sim} \dot{\sim} \dot{\sim} \dot{v} \dot{\sim} \dot{\sim} \dot{\sim} \dot{\sim} \dot{\sim} \dot{\sim} \dot{N} \dot{m} \dot{\jmath}$

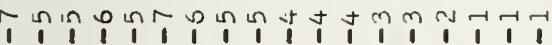

$\neg-4$

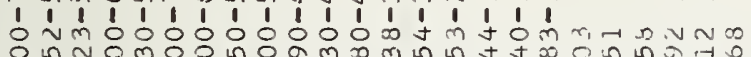

\& $\quad$ in JI t 虫

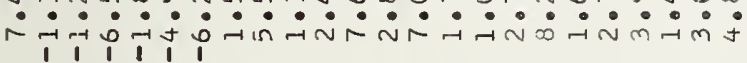
00000 in 00050000000000000

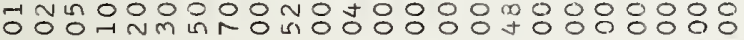

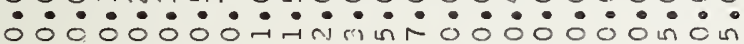
İ

$1 \leftleftarrows 000000000000000000000000$ ニ

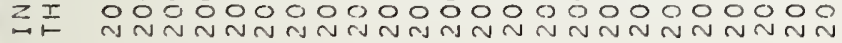

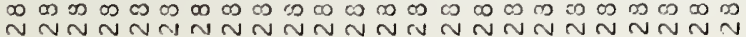

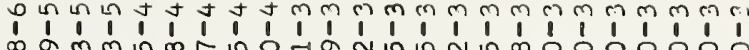

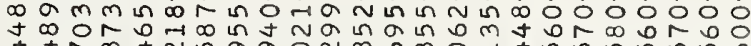
寸

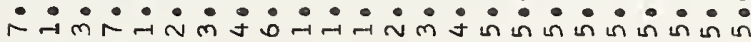

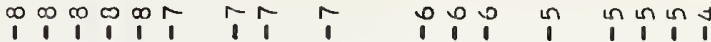

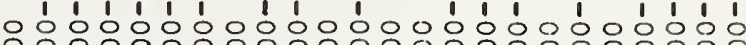

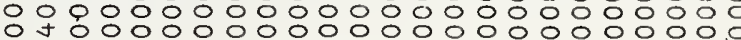

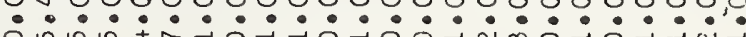
1

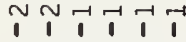

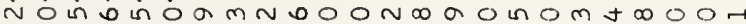

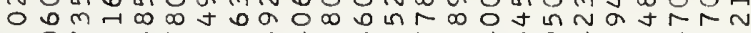
- Or

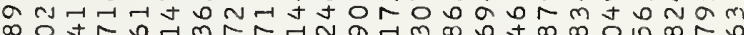
年

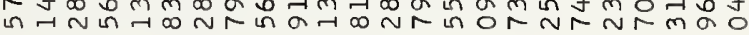

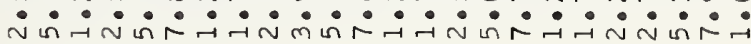

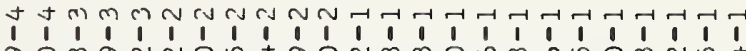

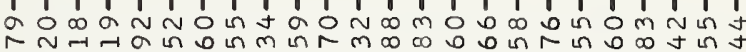

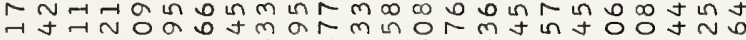

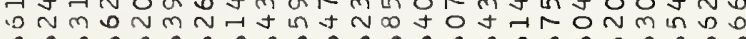

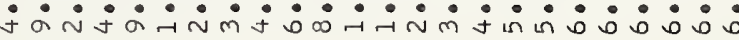

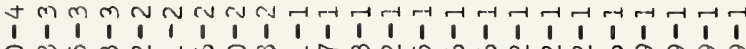

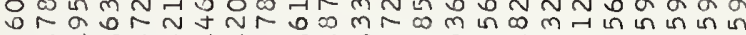

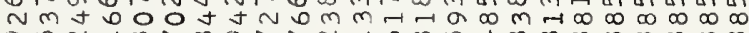

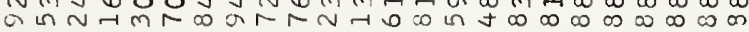

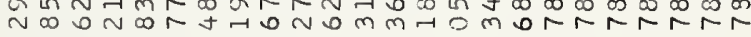

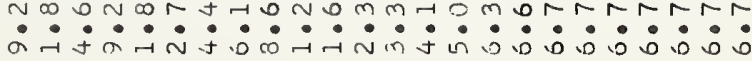

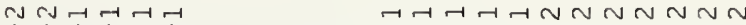

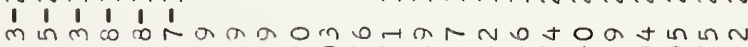

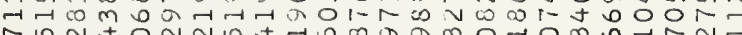

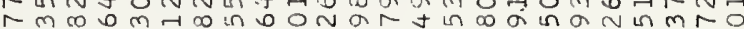

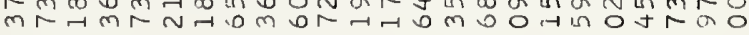

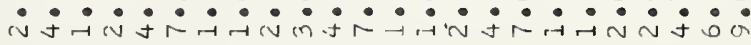

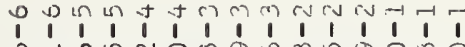

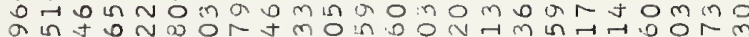

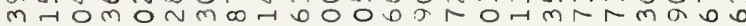
ㄴ.?

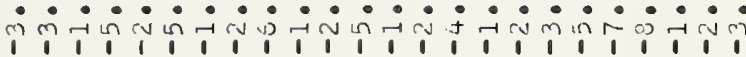
NNNNNNNNNNMNNNMNNNNNNNNN

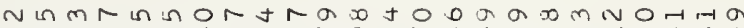

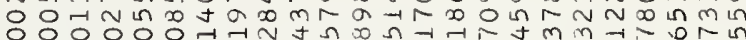

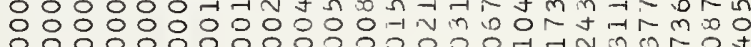

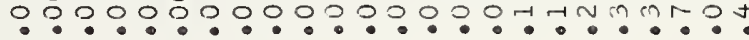

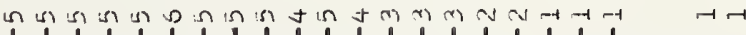

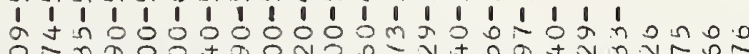

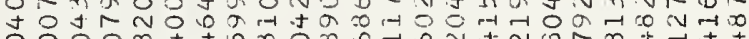
웅… iा

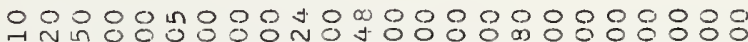

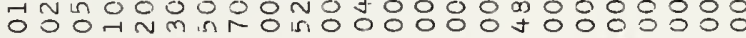
$\dot{0} \dot{0} \dot{0} \dot{0} \dot{0} \dot{0} \dot{\sim} \dot{i} \dot{m} \dot{0} \dot{0} \dot{0} \dot{0} \dot{0} \dot{0} \dot{0} \dot{0}$ $\rightarrow$ n min

000000000000000000000000 $\dot{0} \dot{0} \dot{0} \dot{0} \dot{0} \dot{0} \dot{0} \dot{0} \dot{0} \dot{0} \dot{0} \dot{0} \dot{0} \dot{0} \dot{0} 0 \dot{0} 0:$

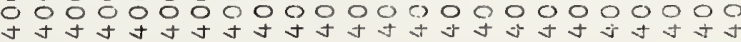

000000000000000000000000 a a a a a $\infty$ O 0 ( $)$ $N N$ N N N N N N N N N N N N N N N N N N N 
N $\begin{array}{llllllllllllllllllllll}1 & 1 & 1 & 1 & 1 & 1 & 1 & 1 & 1 & 1 & 1 & 1 & 1 & 1 & 1 & 1 & 1 & 1 & 1 & 1 & 1 & 1\end{array}$

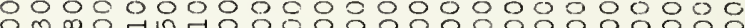

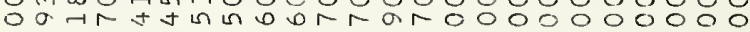

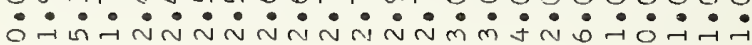

$\begin{array}{lllllll}0 & 1 & 1 & 1 & 1 & 1 & 1\end{array}$

1 แ

m n

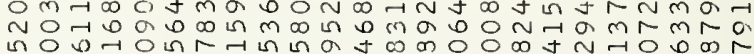

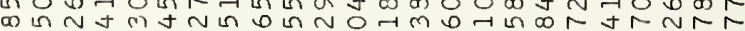

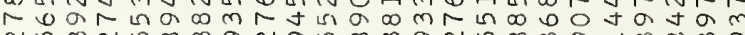

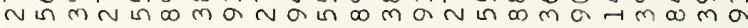

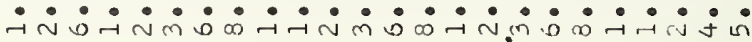

A.4. $\begin{array}{llllllllllllllllllllllll}1 & 1 & 1 & 1 & 1 & 1 & 1 & 1 & 1 & 1 & 1 & 1 & 1 & 1 & 1 & 1 & 1 & 1 & 1 & 1 & 1 & 1 & 1 & 1 \\ 1 & 1 & 0 & 0 & m & m & m & -1 & 0 & 0 & 0 & n & & m & n & 1 & 0 & 0 & 0 & 1 & 0 & 1 & 0 & 0\end{array}$

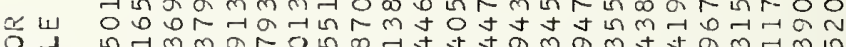

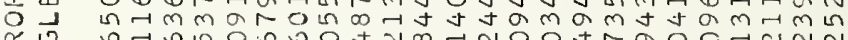

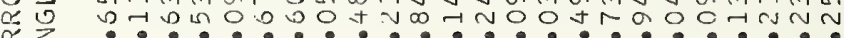

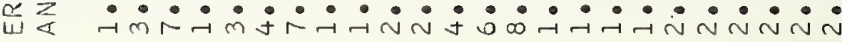

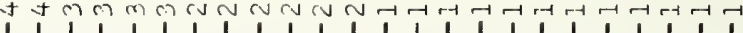
$\begin{array}{llllllllllllllllllllllll}1 & 1 & 1 & 1 & 1 & 1 & 1 & 1 & 1 & 1 & 1 & 1 & 1 & 1 & 1 & 1 & 1 & 1 & 1 & 1 & 1 & 1 & 1 & 1\end{array}$ n m

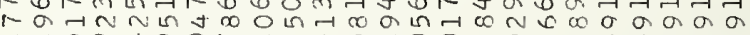
- -1 i

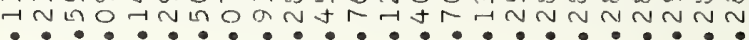

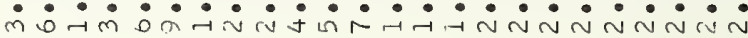

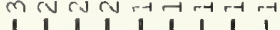

$\sqcap \sqcap \sqcap \sqcap \sqcap \mapsto N N N$

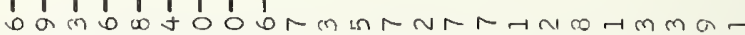

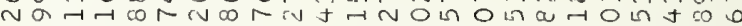
0 in $m$ D. D

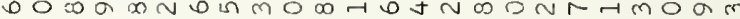

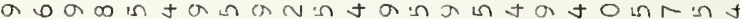

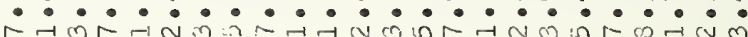

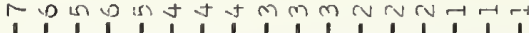

$\mapsto \stackrel{1}{1}$

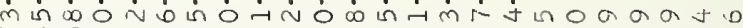

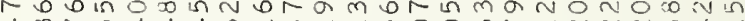
t mN Intat

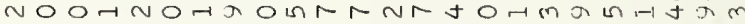

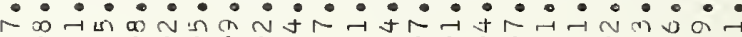

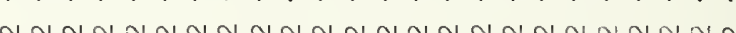

O-ta. 00 a

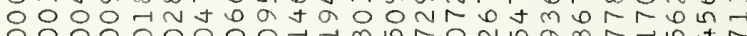
$000000000 \mathrm{0}, \mathrm{m}$.

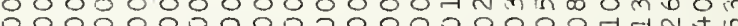

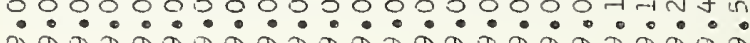

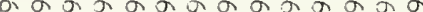

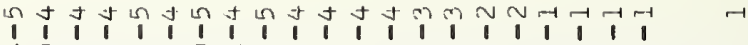

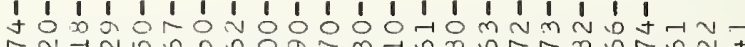

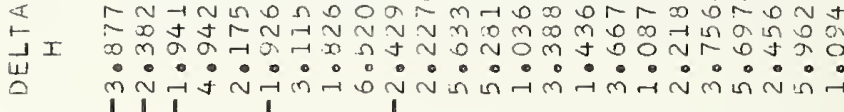
00000 mo I

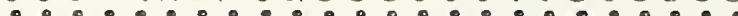

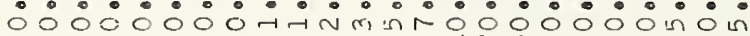
Hom in $\mathrm{N}$ or $\mathrm{N}$ WN

$\leftarrow 5$
$=15$
$=15$

000000000000000000000000

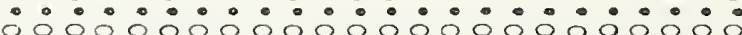

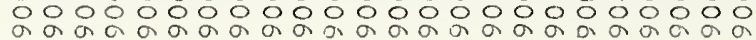

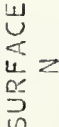

000000000000000000000000

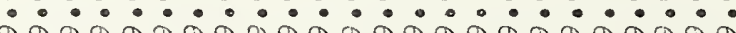
N N N N N N N N N N N N N N N N N N N N N 
$\leqslant$

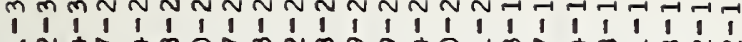

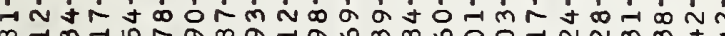

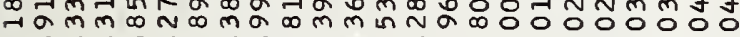

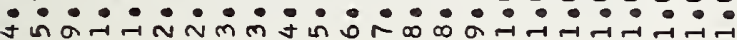

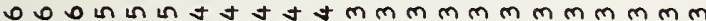
- $\begin{array}{llllllllllllllllllllllllllll}1 & 1 & 1 & 1 & 1 & 1 & 1 & 1 & 1 & 1 & 1 & 1 & 1 & 1 & 1 & 1 & 1 & 1 & 1 & 1 & 1 & 1 & 1\end{array}$

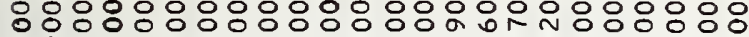

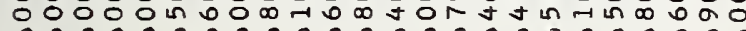

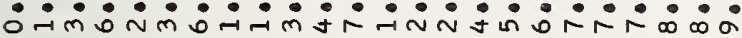

$\pi-\pi-\pi-\pi \sim N N N \sim N N \sim N N \sim m m m m m m$

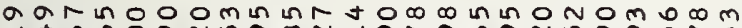

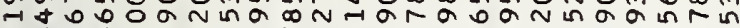
J 0 o

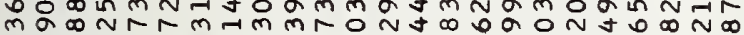
mañâm

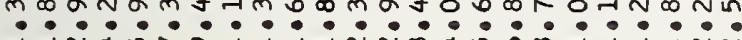
$\dddot{1}$

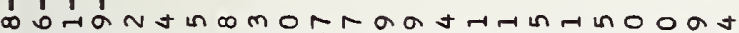

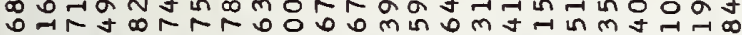

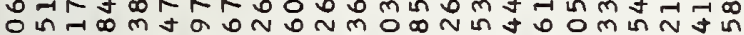

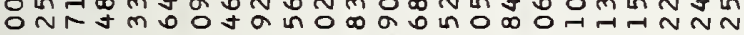

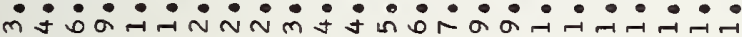

$\because 1$

motm-1 th th matring

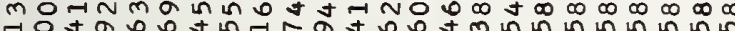

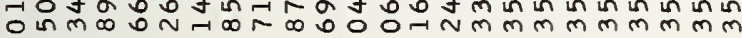

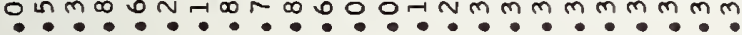

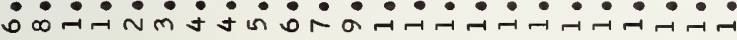

$\rightarrow-r-r-\pi N N N N N N N N N N N m m m m m m$

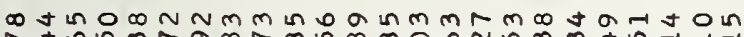
กษ

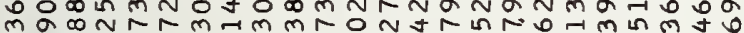
måñom

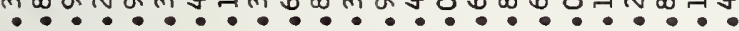

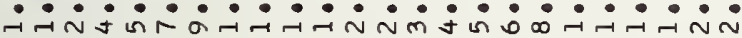

N

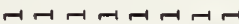

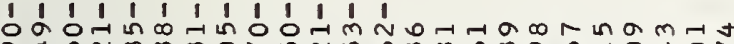

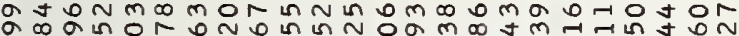
ơ 0 m

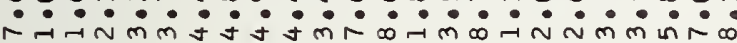
1

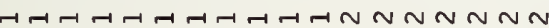
mRminomamm narmamonR MOROA

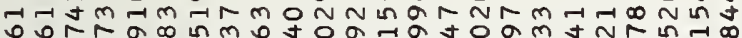
oㄴ

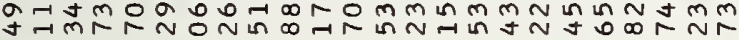

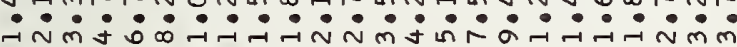

tmmm $m$ m

$r \rightarrow r \rightarrow n$

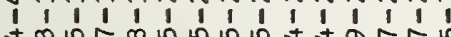

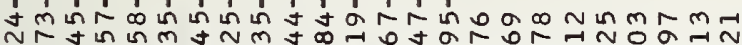

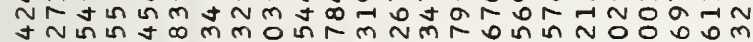
$\rightarrow 0$ 인

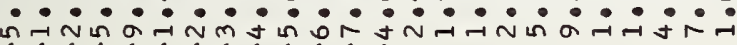
$111,1,1,1,1,1\}$

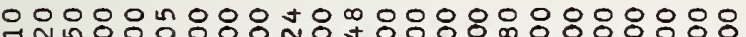

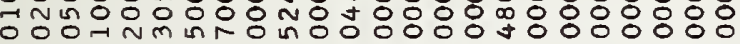

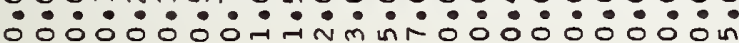

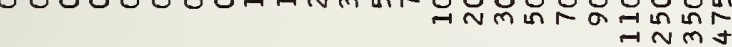

000000000000000000000000

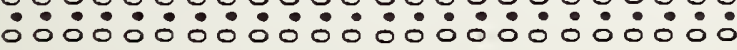

m

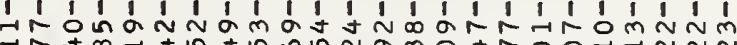

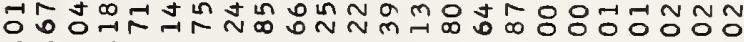

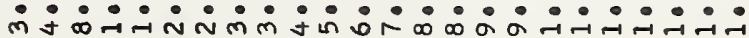

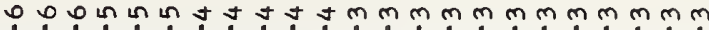

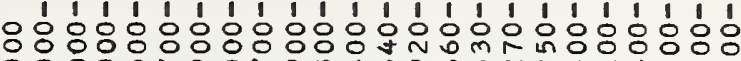

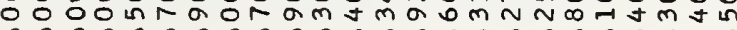

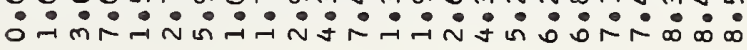

-1-1- -1 N N N N N N N N N m mmmm

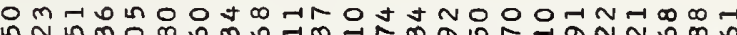

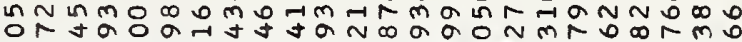

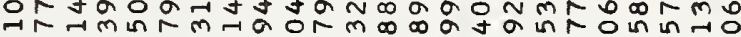

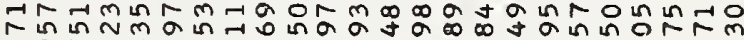

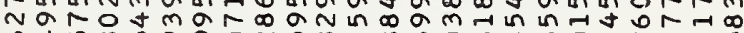
ơ in

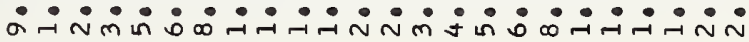

$\overrightarrow{1}$

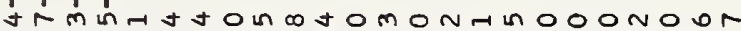

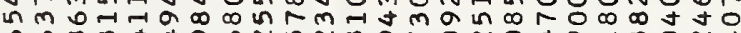

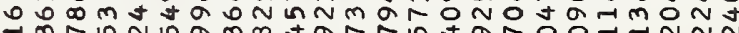

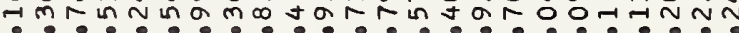

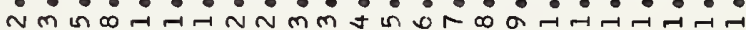

17

1.

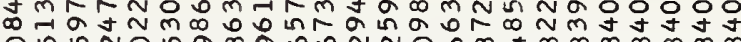

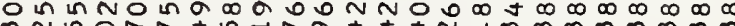

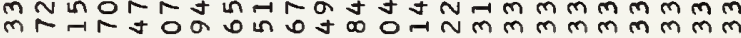

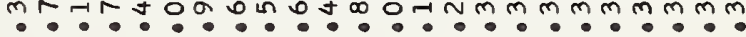

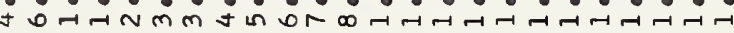

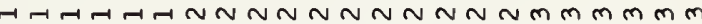

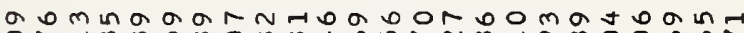

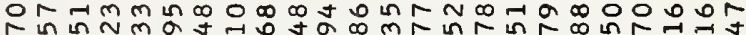
กิ v

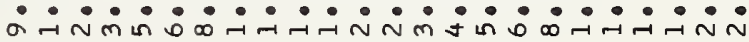

NN J J I

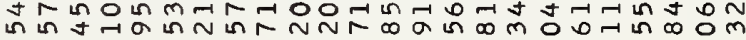

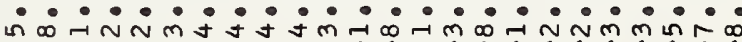
-

otror OrN ty

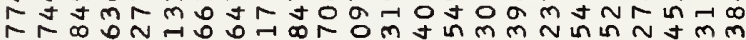

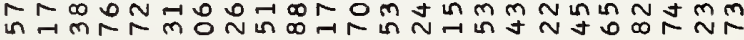

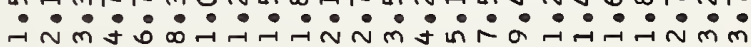

A $m m m N N N N N N N N N \frac{1}{1}$

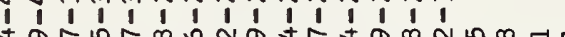

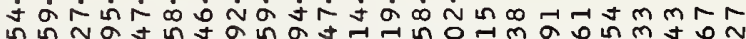

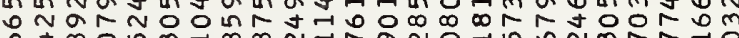

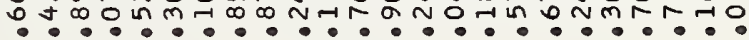

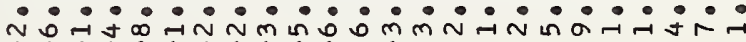
$\begin{array}{lllllllllllll}1 & 1 & 1 & 1 & 1 & 1 & 1 & 1 & 1 & 1\end{array}$

00000 in o o otomo00000000000

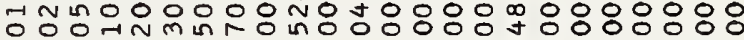

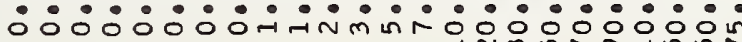

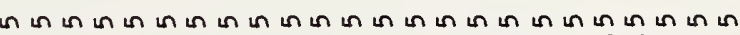

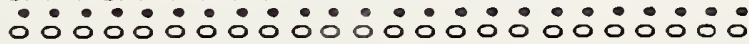

000000000000000000000000

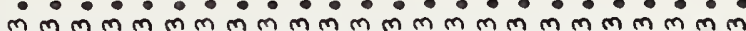

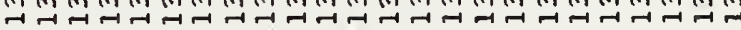

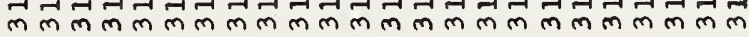

mm 


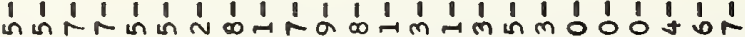

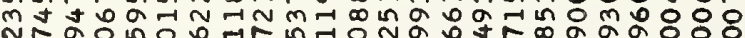
- o o

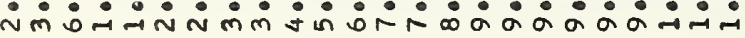

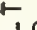
$\overrightarrow{0}$

lin

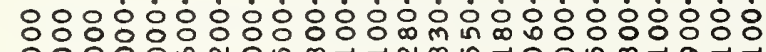

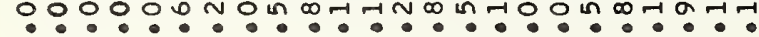

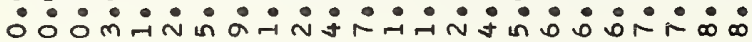

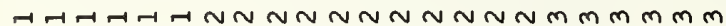

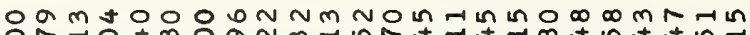

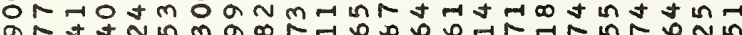

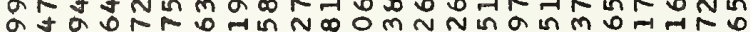
a

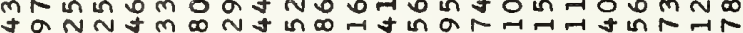

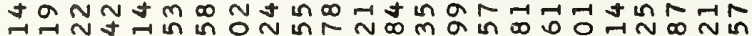

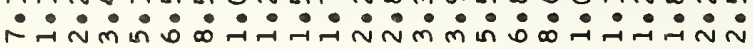

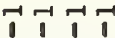

$-1-1-1-1$

$\propto \omega$

DrNMNN

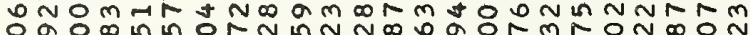

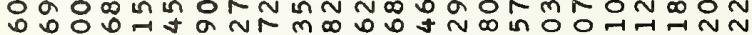

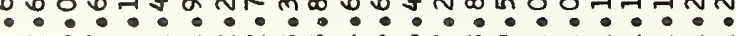

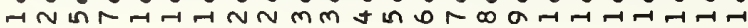

II

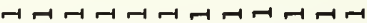

$\infty N r+n-\infty \omega \infty m+a \infty+\infty m \infty n m m$

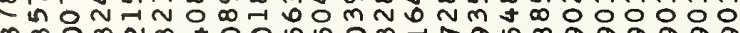

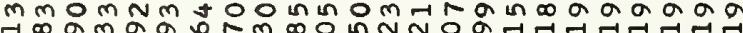

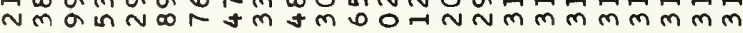

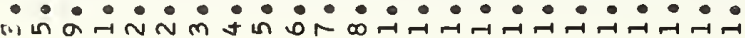

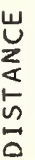

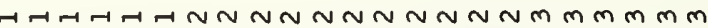
N N N

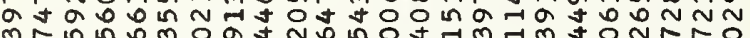

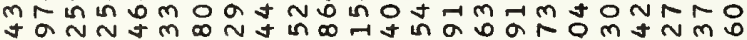

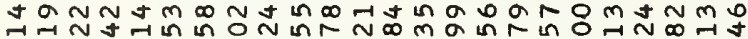

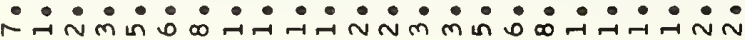

N N-THन-

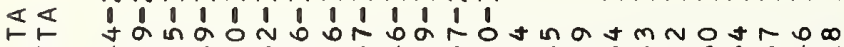

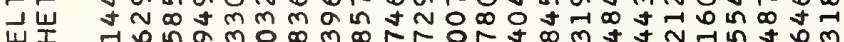

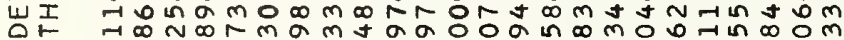

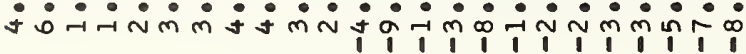

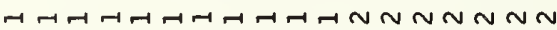

$E$
I
$上$

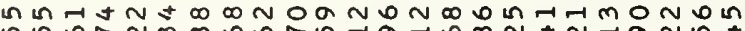

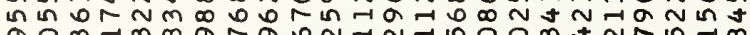

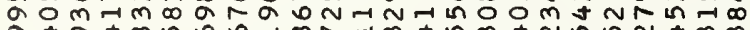

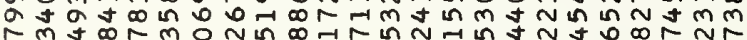
-

$\operatorname{tamm} m \sim N \sim N N N N$

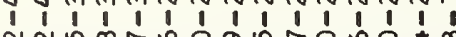

$\varangle$

$0+\pi 0^{2}$

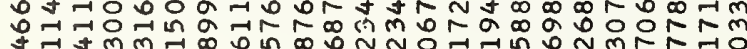

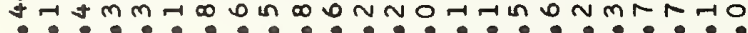

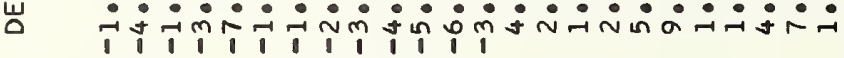

$00000 \mathrm{~m} 000$ t 0 mo 00000000000

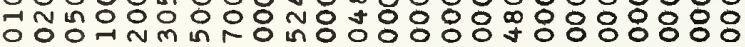

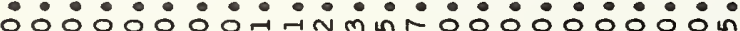

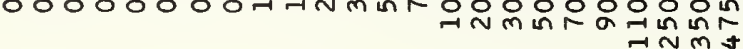

トヒ そ岌

U $\approx>$ mm $m N N N N N N N N N N N N N N N$

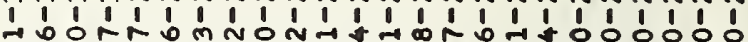

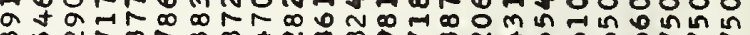

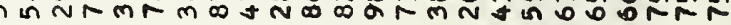
-

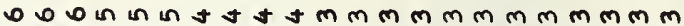

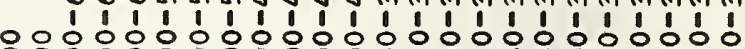

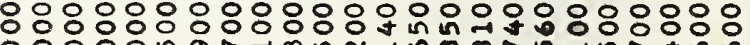

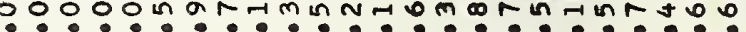

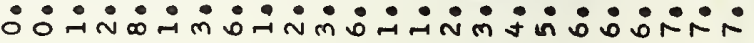

- $-1-H N N N N N N N$ N N

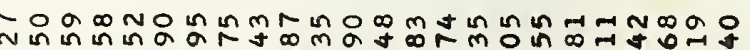

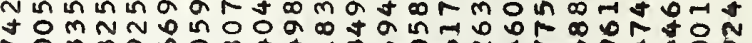

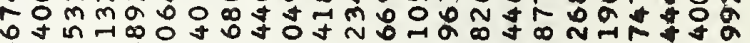

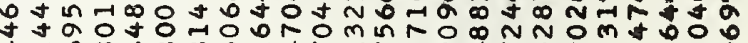

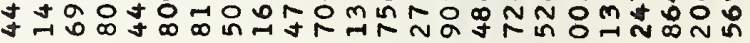

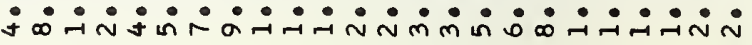

-1

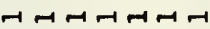

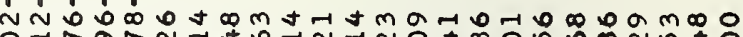

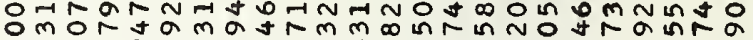

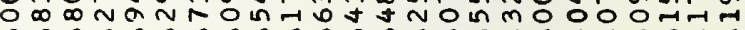

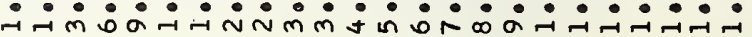

$\overrightarrow{1} \overrightarrow{1}$

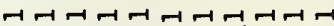

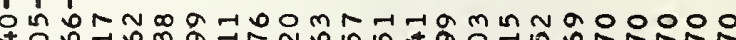

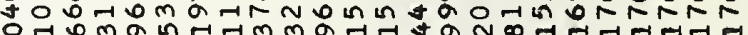

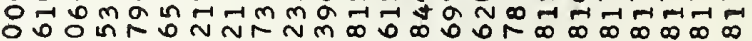

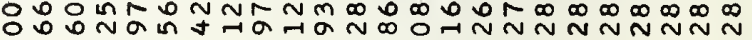

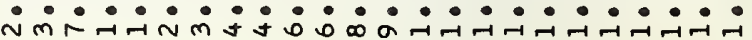

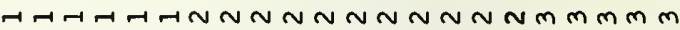

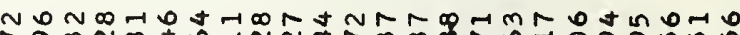
$\sim a m N \infty+0, N N \infty N m \infty d N$

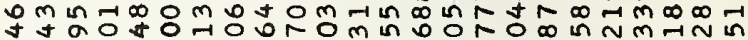

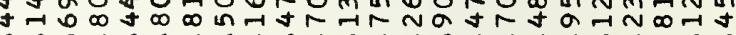

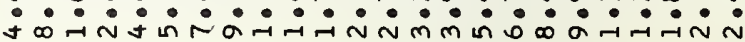

N N

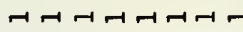
$\begin{array}{llllllllllllll}0 & 1 & 1 & 1 & 1 & 1 & 1 & 1 & 1 & 1 & 1 & 1 & 1 & 1\end{array}$

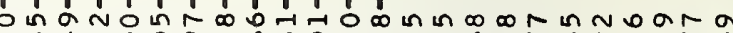
- $\begin{aligned} & 4 \\ & 0\end{aligned}$

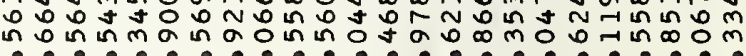

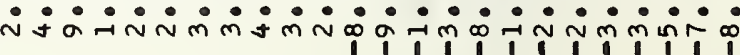

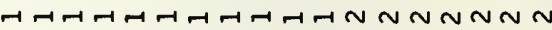

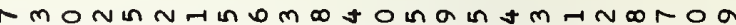

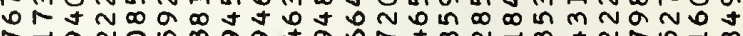

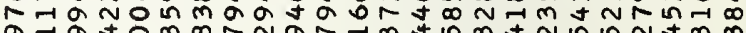

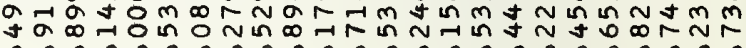

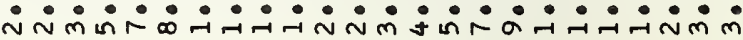

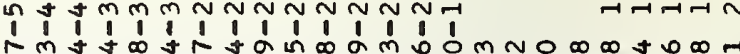
नПमП

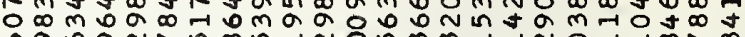

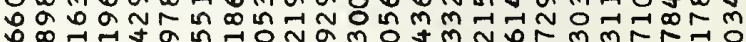

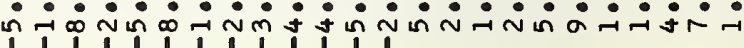

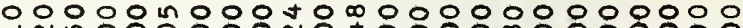
-

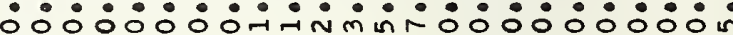

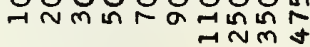

000000000000000000000000

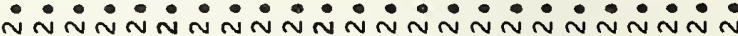

000000000000000000000000 $\ddot{m} \dot{m} \dot{m} \dot{m} \dot{m} \dot{m} \dot{m} \dot{m} \dot{m} \ddot{m} \dot{m} \dot{m} \dot{m} \dot{m} \dot{m} \dot{m} \dot{m}$

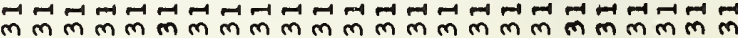


i

$\ll$

I我

岁

枈岁

ज这

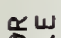

$\frac{\alpha}{\alpha} \frac{0}{2}$

w<

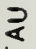

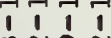

mán

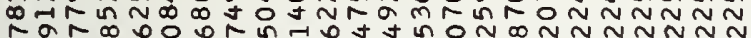

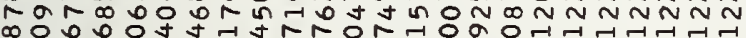

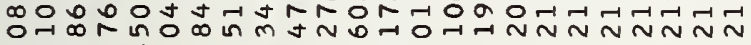

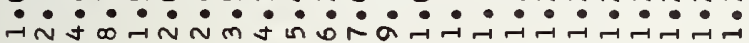

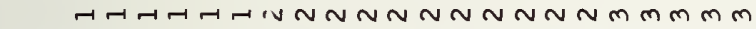

$\sim m m \sim-1 m+m m \infty m m m m \infty$

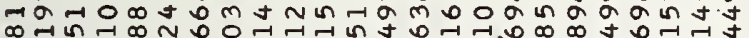

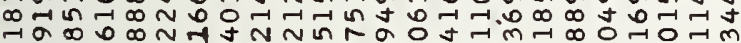

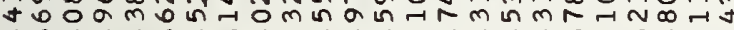

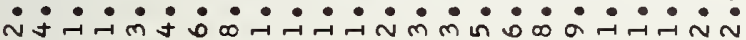

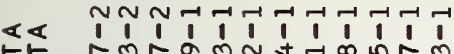

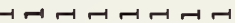

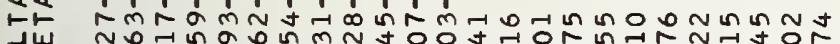

㟧 mo 0 ON

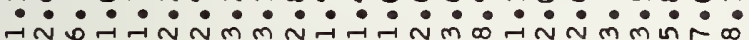

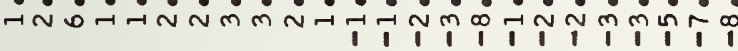
-

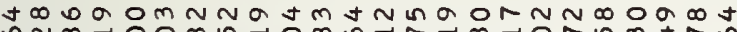

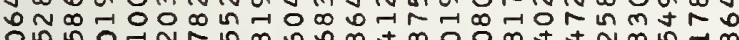

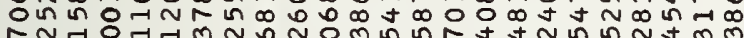

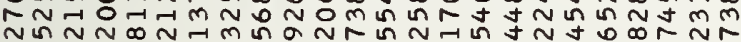
j

m n

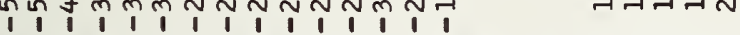

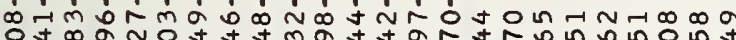
$\ll$

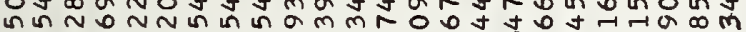
II

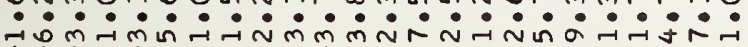
1 i î́

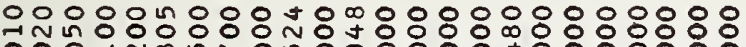
$000 \pi n m$ m

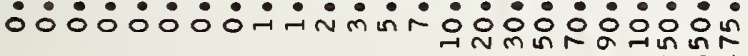
$\rightarrow \pi m$ a

\& 000000000000000000000000

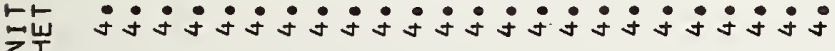

t

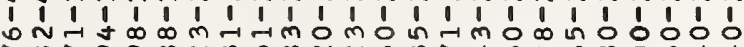

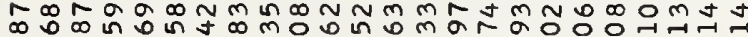

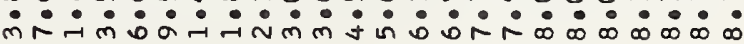

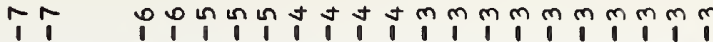

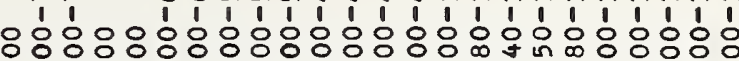

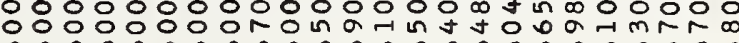

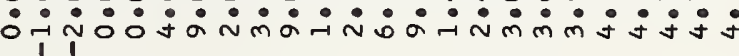

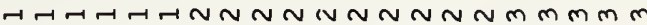

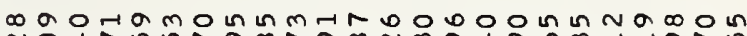
Notrin NRN N $N$ N NT

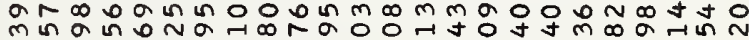

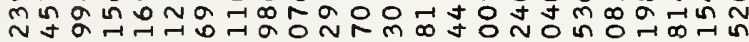

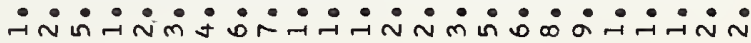

NT

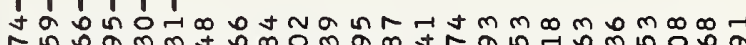

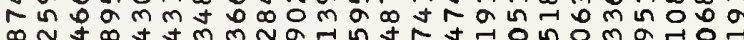

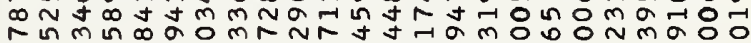

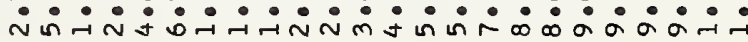

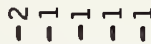

ด 0 แก Tr.t.

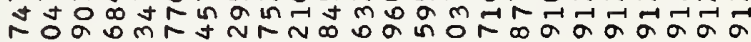
in $-10-10 m 00 m y-t a \alpha \infty 000000000$

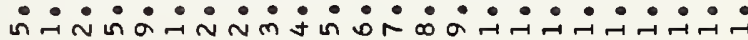

$m \infty m N-1 m 0$ tatona-100Nmm-thor

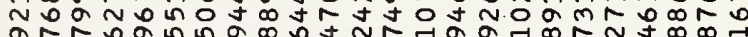
mina

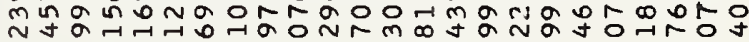

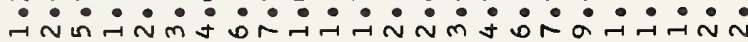

iิ

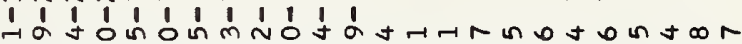

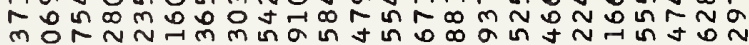

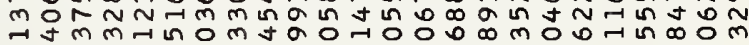

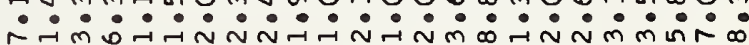
i

A-

a n $\infty N \infty$ n $n$ n t t

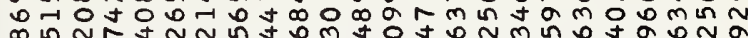
$\infty$ in N mNNo

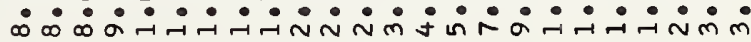

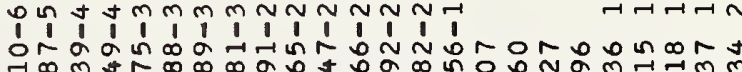

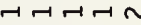

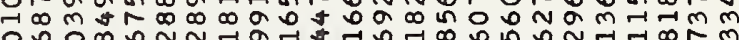

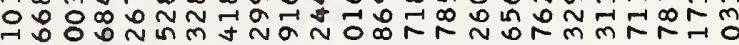

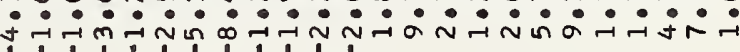
$1,1,1,1,1,1$

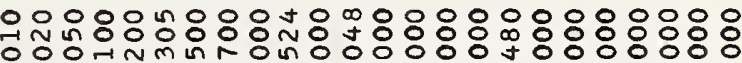

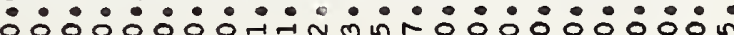

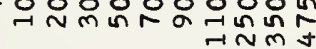

000000000000000000000000 $\therefore \dot{\infty} \dot{\infty} \dot{\infty} \dot{\infty} \dot{\infty} \dot{\infty} \dot{\infty} \dot{\infty} \infty \dot{\infty} \infty \dot{\infty} \dot{\infty} \dot{\infty} \infty \dot{\infty} \infty \dot{\infty}$

000000000000000000000000

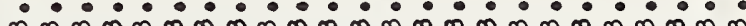

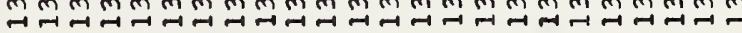

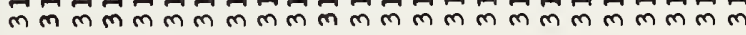




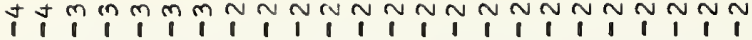

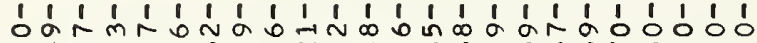
-1 O ư

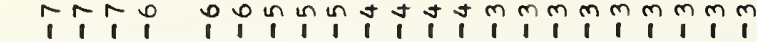
« ur $\alpha 0000000000$

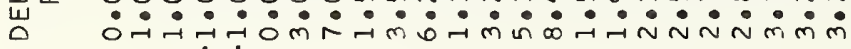
H-H-H-HNNNNNNNNmmmmm If NoO 0 i

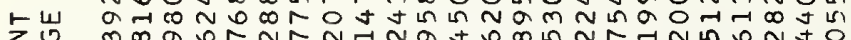

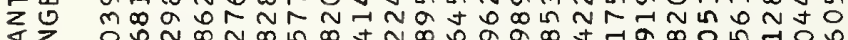
दz $20 \Omega N \infty N \infty$ L

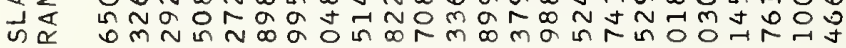

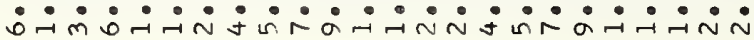

INT

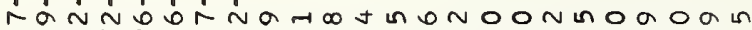

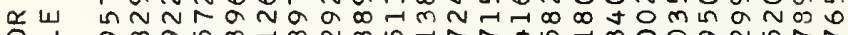

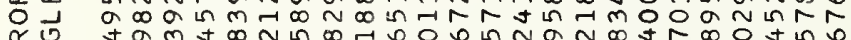

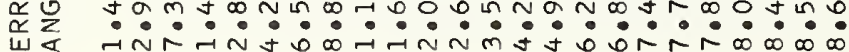

N $N+1+1$

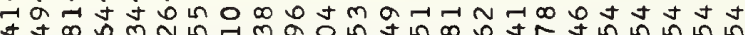

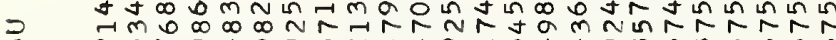

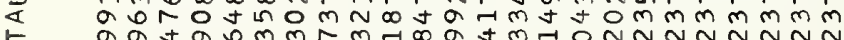
- a

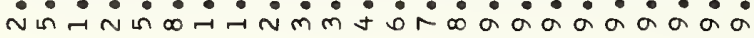

$\frac{u}{2}$

1

mo comat 0 N

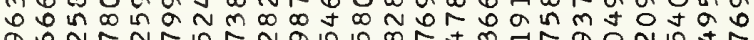
a b N N

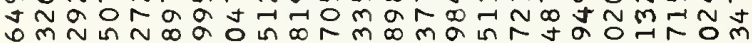

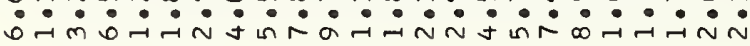

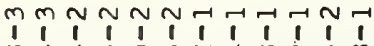

$\varangle$

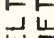

$\infty$ t Na $\infty$ in $\infty$ in : $m \sim-m$ ம

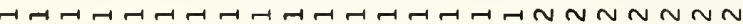

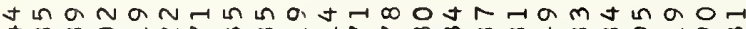

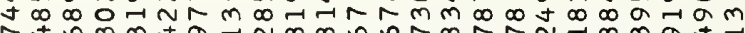

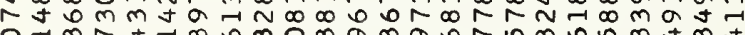

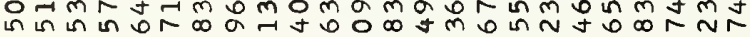

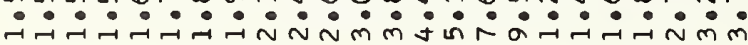

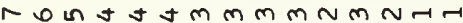

$\begin{array}{llllllllllllllll}1 & 1 & 1 & 1 & 1 & 1 & 1 & 1 & 1 & 1 & 1 & 1 & 1 & 1 & 1 & 0\end{array}$ 잉 9 m

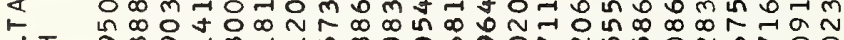

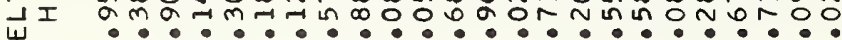

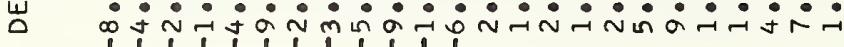

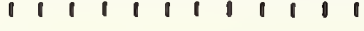

$\frac{5}{5}$

00000 L 000 * 0000000000000 oñ

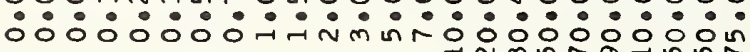

15000000000000000000000000

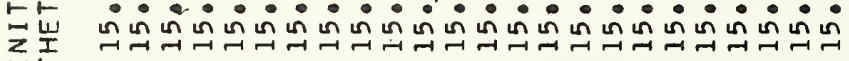

岁 $\ll z$ $\frac{u}{2} \geq$

000000000000000000000000 $\dot{m} \dot{m} \dot{m} \dot{m} \dot{m} \dot{m} \dot{m} \dot{m} \dot{m} \dot{m} \dot{m} \dot{m} \dot{m} \dot{m} \dot{m} \dot{m} \dot{m} \dot{m}$

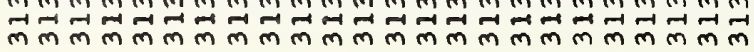

A t

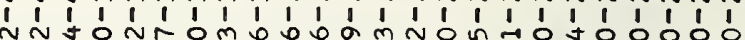

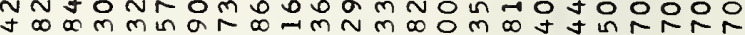
to $\dot{\sim} \dot{\sim} \dot{i} \dot{i} \dot{m} \dot{j} \dot{0} \dot{a} \dot{\sim} \dot{\sim} \dot{m} \dot{m} \dot{j} \dot{j} \dot{j} \dot{j} \dot{j} \dot{j} \dot{j} \dot{j}$

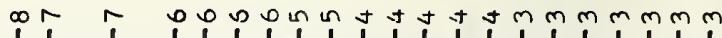

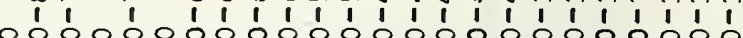
응ㅇㅇㅇㅇㅇㅇㅇㅇㅇㅇㅇㅇㅇㅇㅇㅇㅇㅇㅇㅇㅇㅇㅇㅇㅇㅇㅇㅇㅇㅇㅇㅇㅇㅇㅇㅇㅇㅇ $00000000000000 \mathrm{~m} 0 \mathrm{~m} 0 \mathrm{n} \mathrm{m} \mathrm{m} \mathrm{m}$

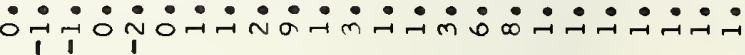
ît $\pi \rightarrow-r-\sqcap N \sim \sim N \sim \sim N \sim m m m$

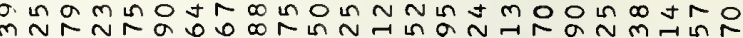

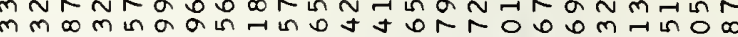

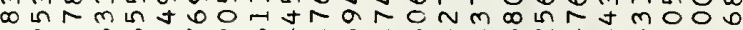

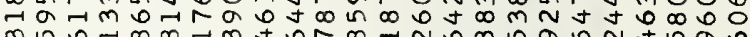

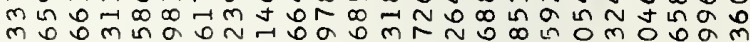

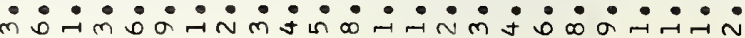

MT

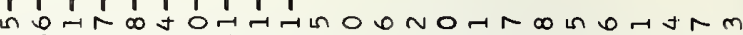

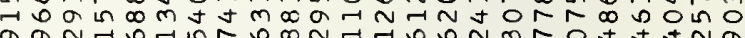

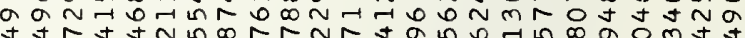
ง.? J.?

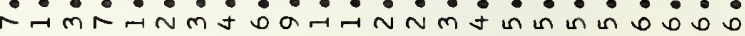

$N N N-1-1+1$

$\begin{array}{lllllllllll}2 & 1 & 1 & 1 & 1 & 1 & 1 & 1\end{array}$

a HOJ

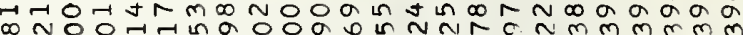

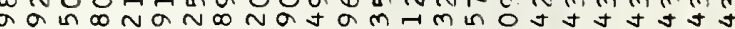

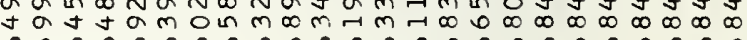

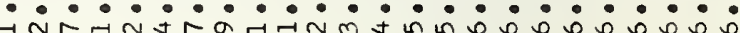

17

Hन

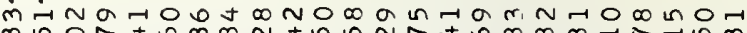

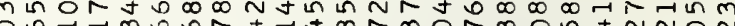
OD $m \leftarrow 0-\infty N-m$ or

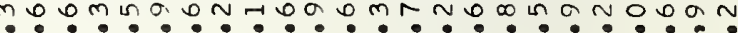

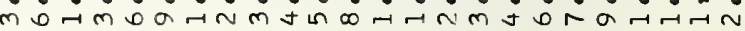

$m m m \sim N N \sim N N N m-7$

-

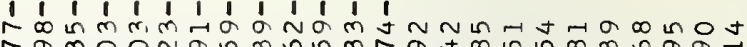

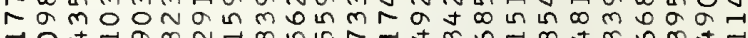

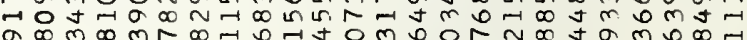

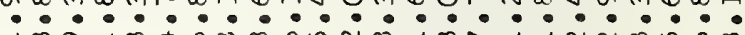

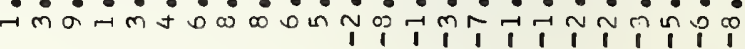

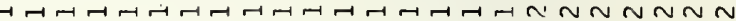

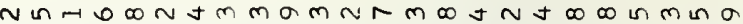
Nab-100 t $N m m$ m Om J J

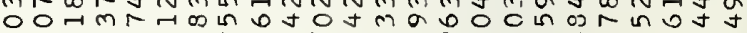

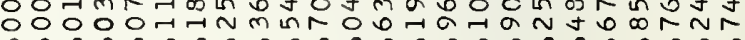

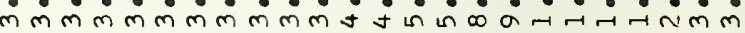

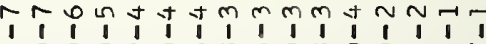

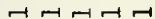

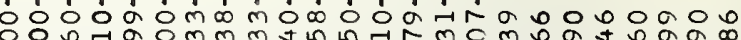
min

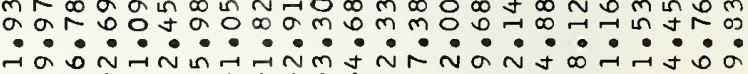

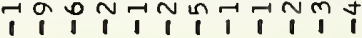

00000 L 00070 mo 00000000000

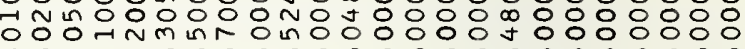
은?

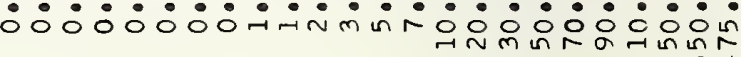

000000000000000000000000

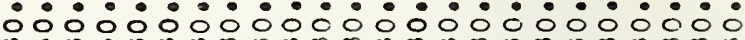
m m m m m m m m m m m m m

000000000000000000000000 $\dot{m} \dot{m} \dot{m} \dot{m} \dot{m} \dot{m} \dot{m} \dot{m} \dot{m} \dot{m} \dot{m} \dot{m} \dot{m} \dot{m} \dot{m}$ $\vec{m} \vec{m} \vec{m} \vec{m} \vec{m} \vec{m} \vec{m} \vec{m} \vec{m} \vec{m} \vec{m} \vec{m} \vec{m} \vec{m} \vec{m} \vec{m}$ 
แn

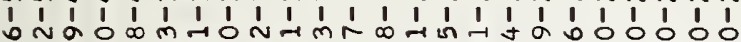

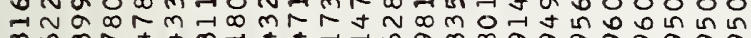

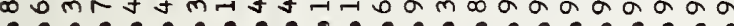

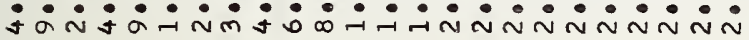

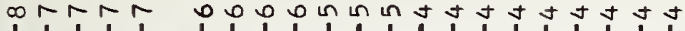
$\ll$ $\mp \propto$ $\vec{\omega}$

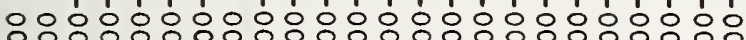
o 000000000

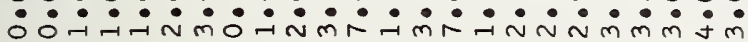

$7 \rightarrow-1$

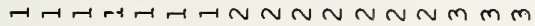

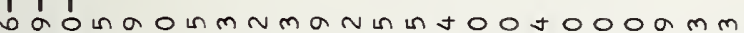
N

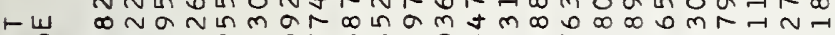
之U

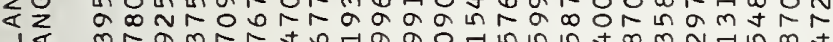

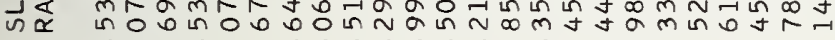

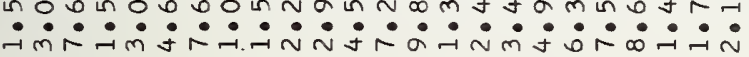

$m m \sim \approx n-1-1-1-1$

o d a m

r. \&u

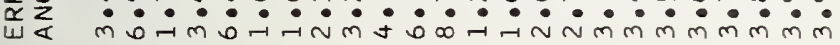

$m \sim N \sim-1-1-1+1$

ta年 J N O

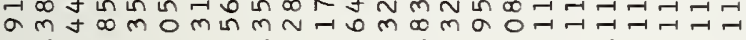

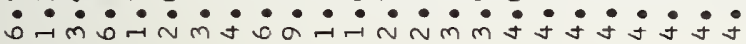

$-1+1$

$-\sqcap--1-1 \sim \sim \sim N \sim \sim N m m m$

t $m m \sim \sim \sim N \sim N m-1$

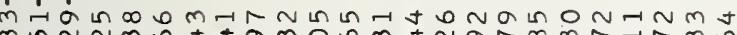

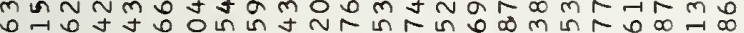
mたn

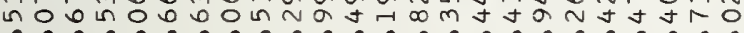

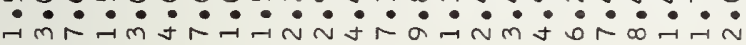

$\leftarrow$

岂岌

OF

$\ll$

$\frac{W}{I}$

$\stackrel{I}{I}$

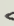

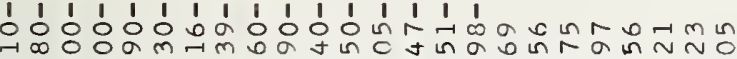
JI II

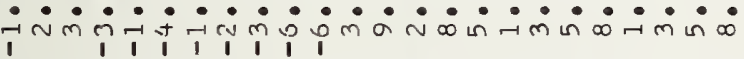

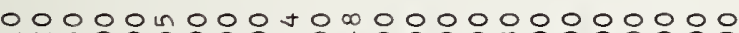
I

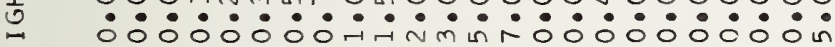
I

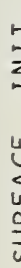

- 100000000000000000000000

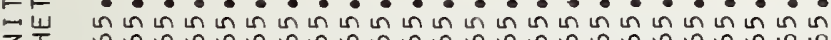

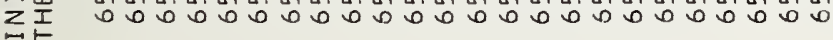

m n t t t t + t

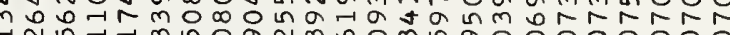
는?

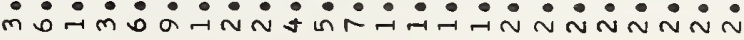

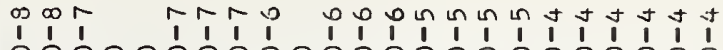

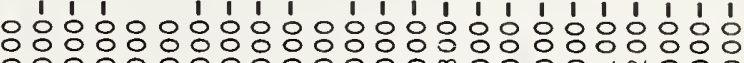
$00000000000000 \infty 0000 \pi n 00$

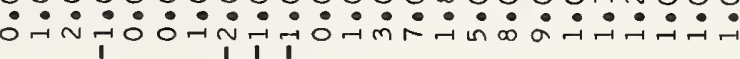

I

$m-1$ in $\infty$ t $N 00 m-10 N 00 N \infty \infty 0$ in $n N \infty N$

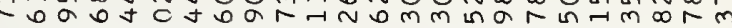
- Lnan arra - inaoram

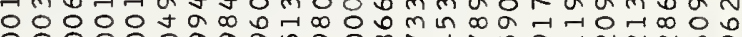
?

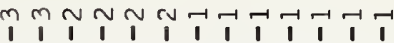

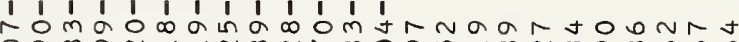

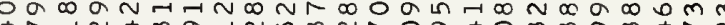
a

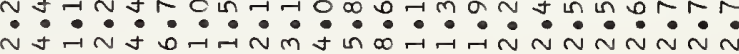

$m m \sim r n \pi-1,1-1,1$

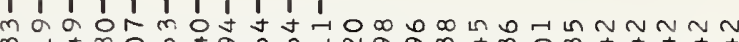

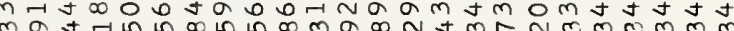

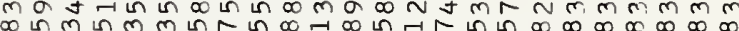

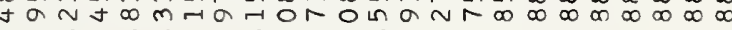

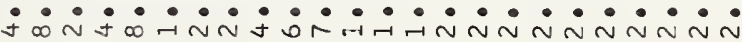

$1+1$

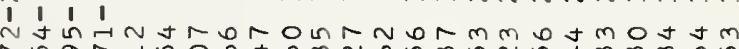
L In $T$ ํㅢ

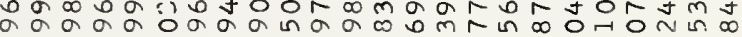

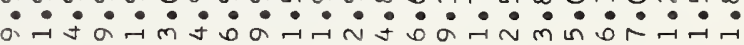

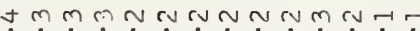

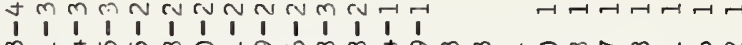

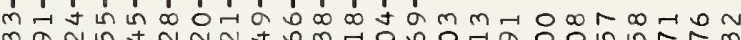
mañ

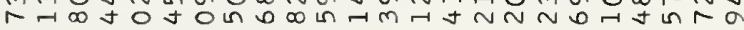
$\begin{array}{llllllllllllll}1 & 1 & 1 & 1 & 1 & 1 & 1 & 1 & 1 & 1 & 1 & 1 & 1 & 1\end{array}$

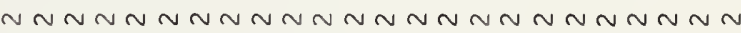

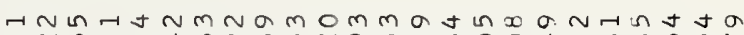
-1 $\sim$ L

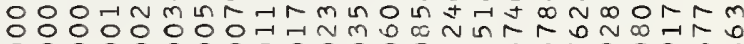
$00000000000000 \mathrm{n} m$ in $\mathrm{r} \alpha 0 \mathrm{~m}^{\circ}$

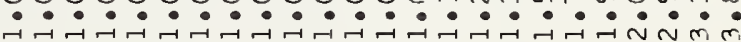

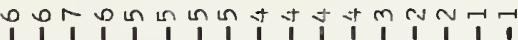

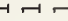

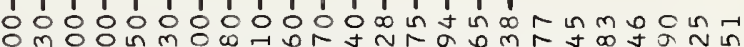

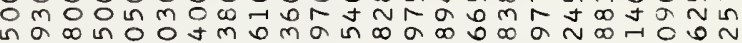

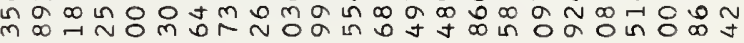

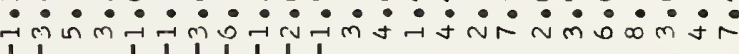
$\begin{array}{lllllllllll}1 & 1 & 1 & 1 & 1 & 1 & 1 & 1\end{array}$

응ㅇㅇㅇㅢ

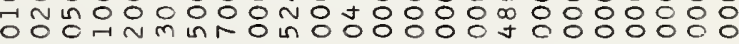

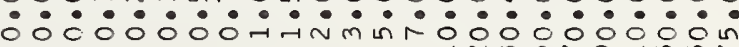

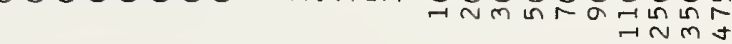

000000000000000000000000 $\dot{0} \dot{0} \dot{0} \dot{0} \dot{0} \dot{0} \dot{0} \dot{0} \dot{0} \dot{0} \dot{0} \dot{0} \dot{0} \dot{0} \dot{0} \dot{0} \dot{0} \dot{0} \dot{0} \dot{0}$ 0000000000000000000000000

000000000000000000000000 m $\dot{m} \dot{m} \dot{m} \dot{m} \dot{m} \dot{m} \dot{m} \dot{m} \dot{m} \dot{m} \dot{m} \dot{m} \dot{m} \dot{m} \dot{m}$ m 


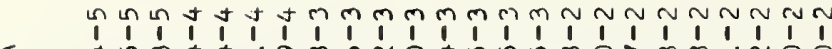

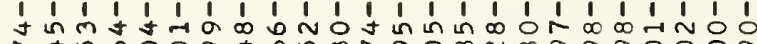

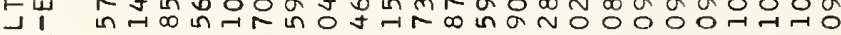

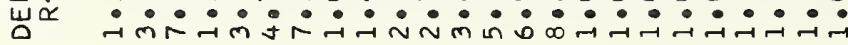

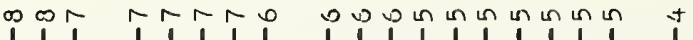

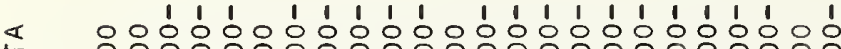
$\mu \alpha \quad 000000000009000000000000$

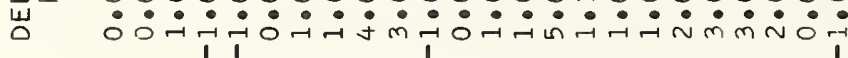
$\stackrel{1}{1} \overrightarrow{1}$

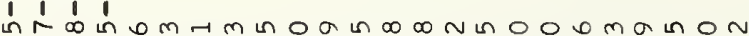

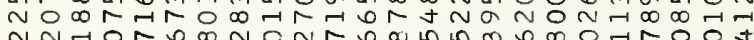
ᄂ $N$ N

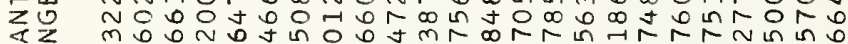

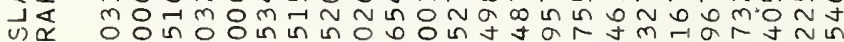

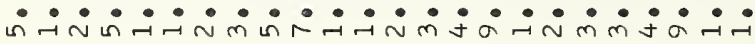

mminan

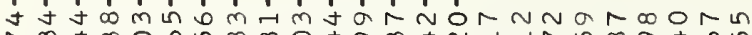
땐 $r m y m 00$ in

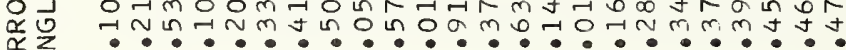
w⿺

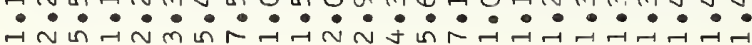

mmiñ

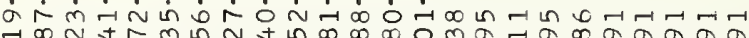
la $\quad$ J $n+\pi n m b 0 y 00 \infty$ y

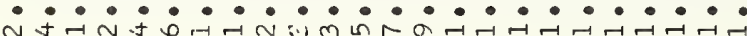

$U$
$z$
in
in
0

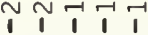

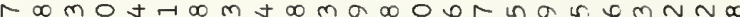

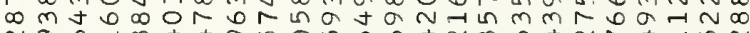

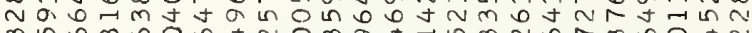

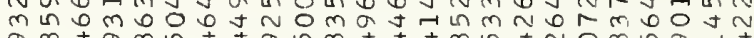

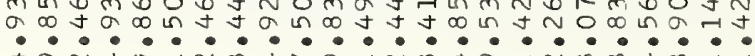
a

$\ll$

岌

A t m m m m n

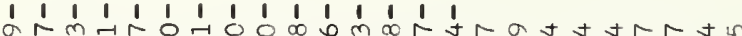

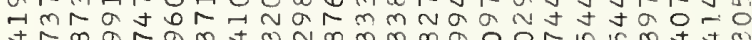

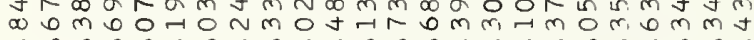

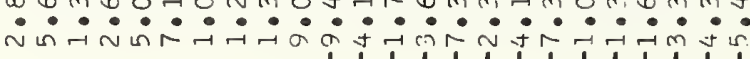

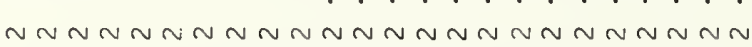

un $\rightarrow$ L $0 \pi n$ n

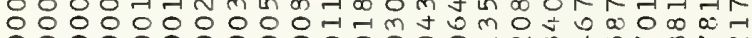

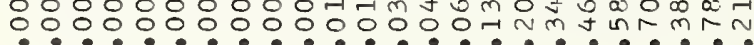
$\dot{\sim} \dot{\sim} \dot{\sim} \dot{\sim} \dot{\sim} \dot{\sim} \dot{\sim} \dot{\sim} \dot{\sim} \dot{\sim} \dot{\sim} \dot{\sim} \dot{\sim} \dot{\sim} \dot{\sim} \dot{\sim} \dot{m} \dot{j}$

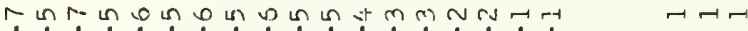

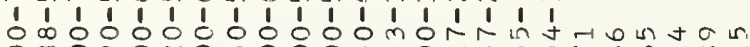

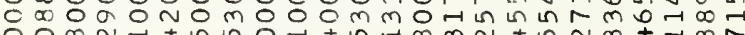

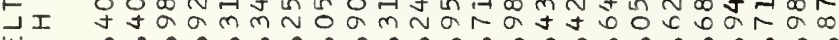

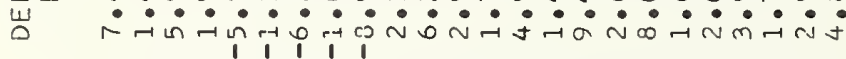
00000 m o o otomo00000000000 I $\stackrel{I}{\Xi}$ I

ᄂ =

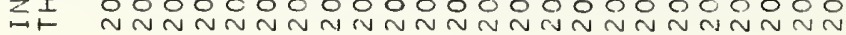

$\omega$

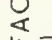

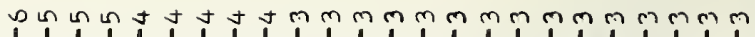
$\begin{array}{llllllllllllllllllllllll}1 & 1 & 1 & 1 & 1 & 1 & 1 & 1 & 1 & 1 & 1 & 1 & 1 & 1 & 1 & 1 & 1 & 1 & 1 & 1 & 1 & 1 & 1 & 1\end{array}$

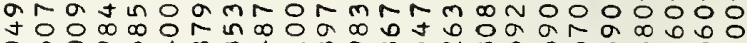

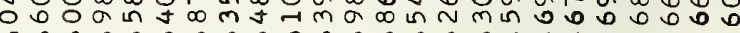

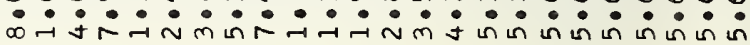

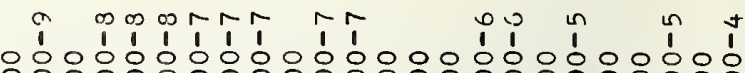

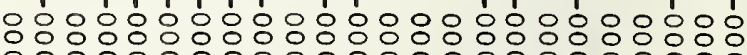
000000000000000000000000

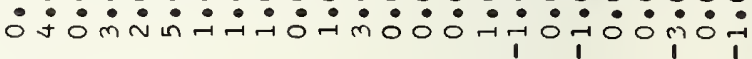

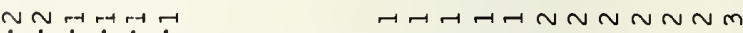
어의

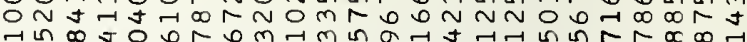
$\neg$ in

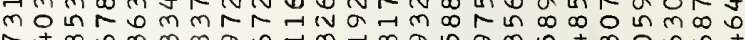

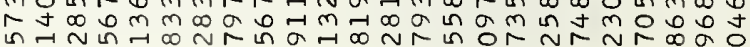

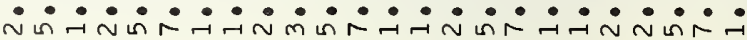

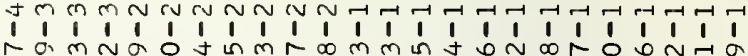

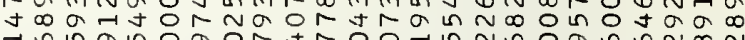

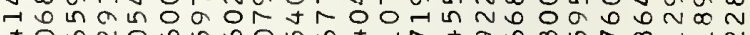
ง? 0 ? 0 in 0 ?

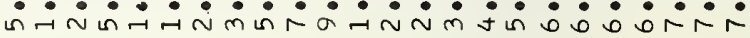

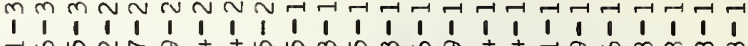
-

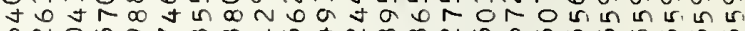

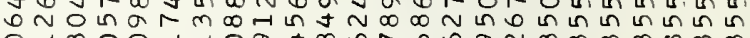

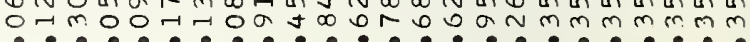

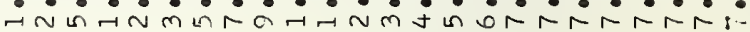

$\hat{1} \hat{1}$

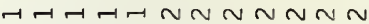

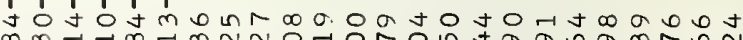

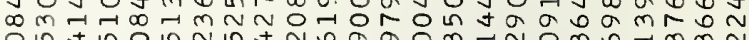

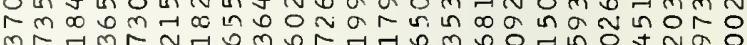
mก na

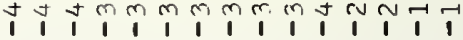

$-1-1$ - i d in

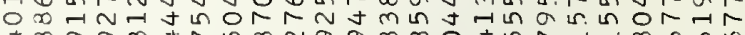

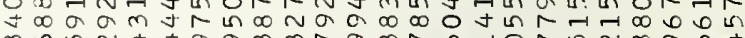

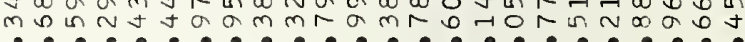

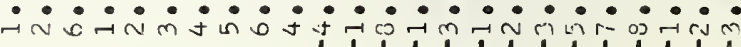
N N N N N N N N N N N N N N N N N

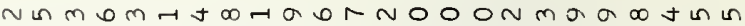
CONNUM⿻上丨

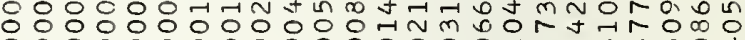
$0000000000000000-1 n m m \infty 04$

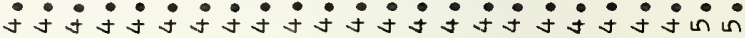

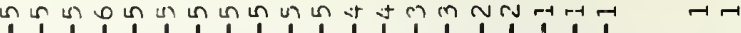

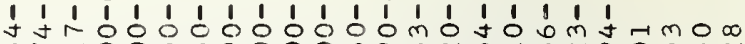
or NoO

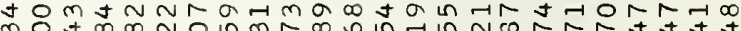

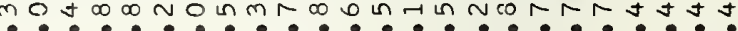

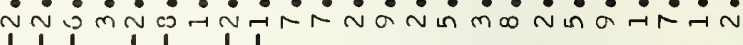

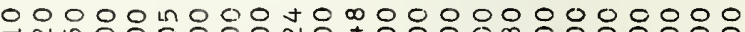

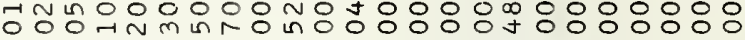

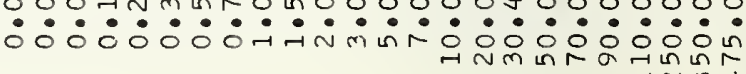

000000000000000000000000 - $\dot{0} \dot{0} \dot{0} \dot{0} \dot{0} \dot{0} \dot{0} \dot{0} \dot{0} \dot{0} \dot{0} \dot{0} \dot{0} \dot{0} \dot{0} \dot{0}$

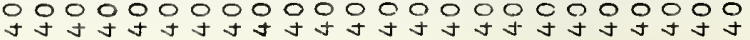

000000000000000000000000 $\dot{m} \dot{m} \dot{m} \dot{m} \dot{m} \dot{m} \dot{m} \dot{m} \dot{m} \dot{m} \dot{m} \dot{m} \dot{m} \dot{m} \dot{m} \dot{m} \dot{m}$

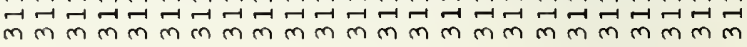




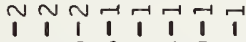

$\neg-\sqcap-\rightarrow \sim N N \sim N$

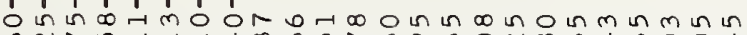

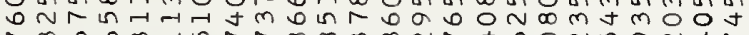

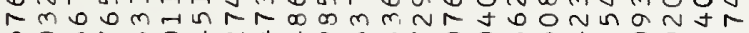

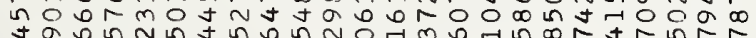
ta d in $n$ in

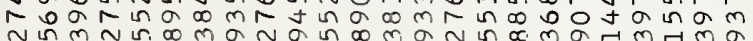

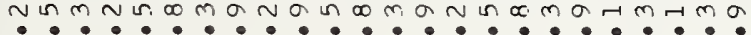

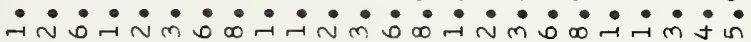

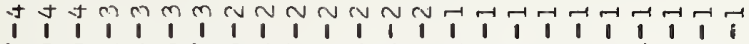

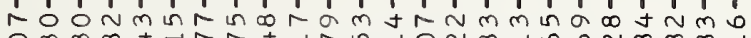

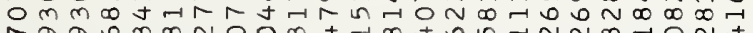

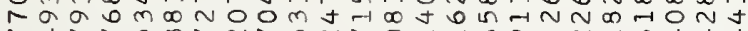

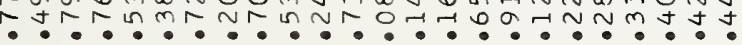

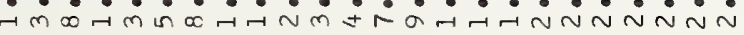

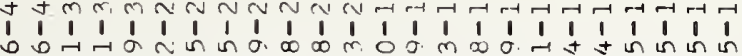

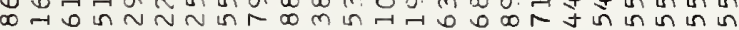

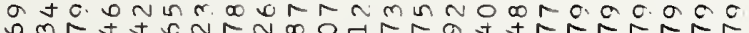

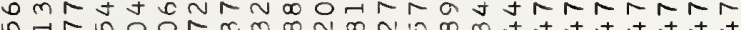
组

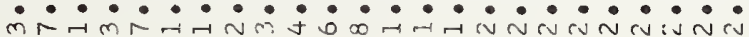

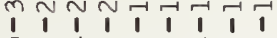

$A \rightarrow H \rightarrow H \rightarrow N N N$

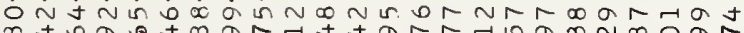

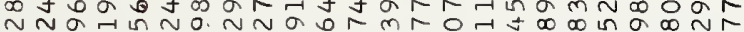

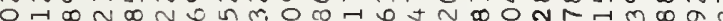
$\alpha$ ba $a$ in $a$ in $\alpha$ in $t a$ in $\alpha$ in $t a+0$ in $\infty$ in $y$

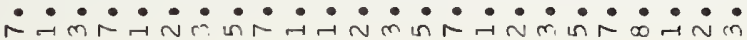

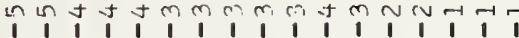

$\overleftrightarrow{\leftarrow \leftrightarrow}$

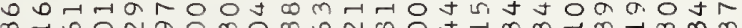
a

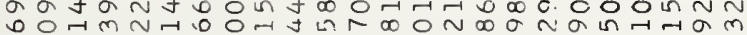

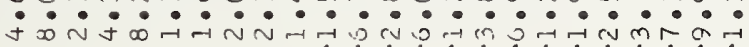

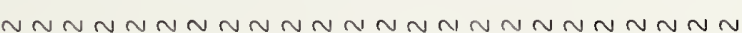

OHanr

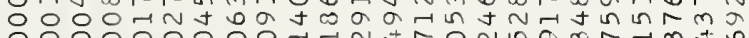
응ㅇㅇㅇㅇㅢ

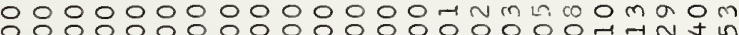

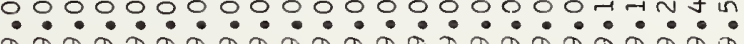

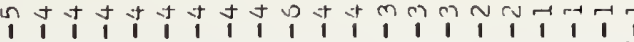

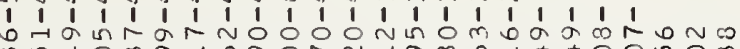

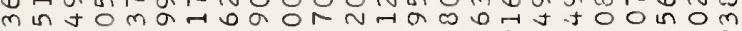

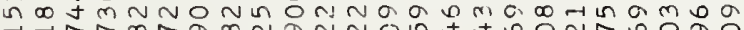
$\because ?$ ก.

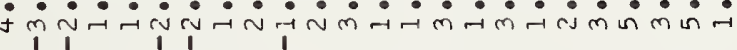

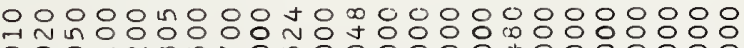
I $000 \pi n m m i o n o 0000070000000$

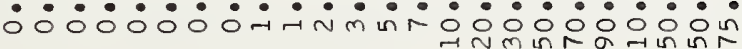
$\rightarrow \mathrm{nJ}$

000000000000000000000000

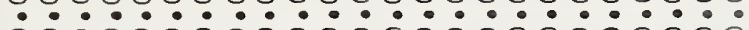

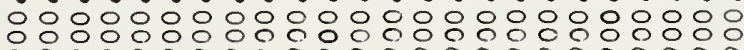

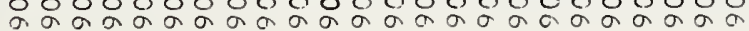

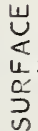

000000000000000000000000 ن

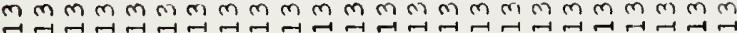

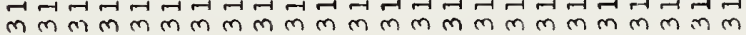


Mิ

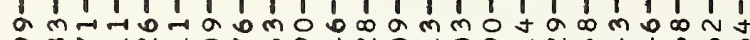

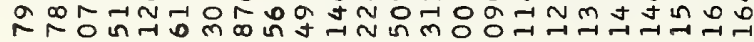
0

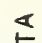
$\vec{w} \propto$ wू

选岕

in $\frac{\pi}{\alpha}$

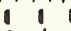

Sin

J tan $n$ in $n$ 告山 O U

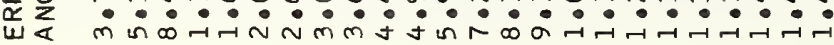

$\overrightarrow{1}$

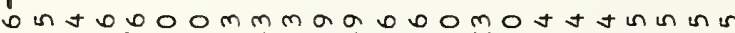

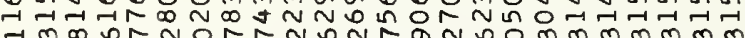
สำ

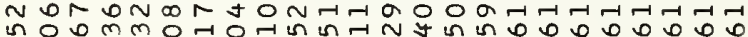

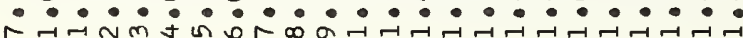

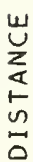

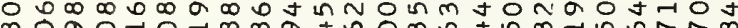

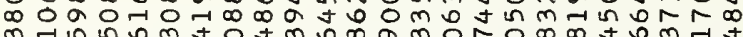

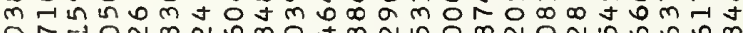

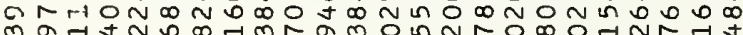

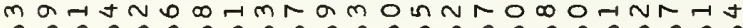

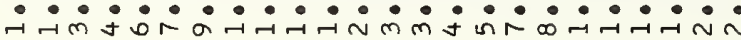

$\leftarrow \leftarrow$

$1+1.14$

$\vec{i}$

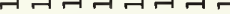

嵌

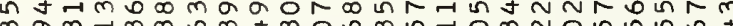

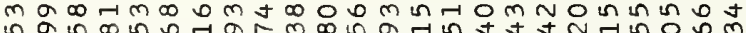

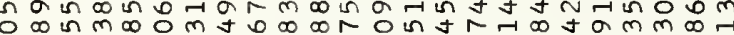

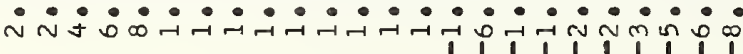

A-

$\underset{⿱ 亠 䒑}{ \pm}$

N

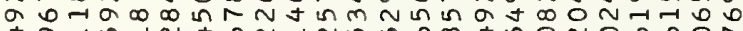

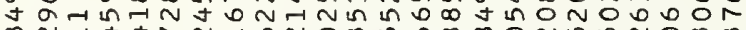
m $N$ T t inj

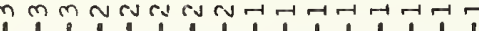

ПナナનП

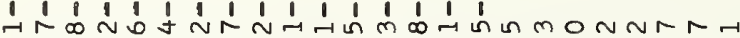

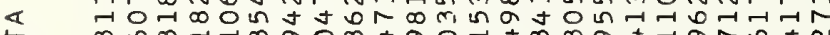

II

岁 m

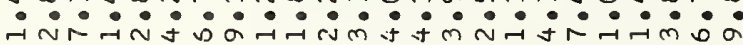
$1,1,1,1,1111111$

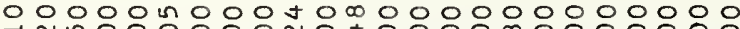
inino

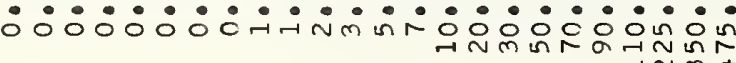

㞬

I 100000000000000000000000 上世 m m m d N N

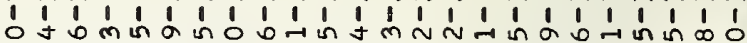
워ำ

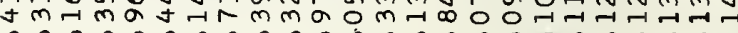

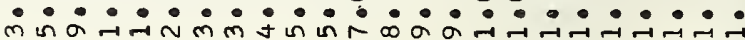

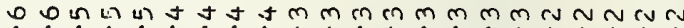

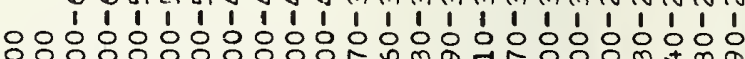

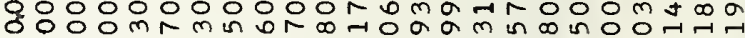

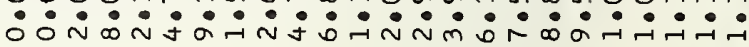

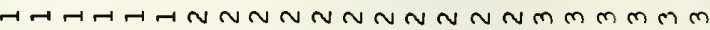

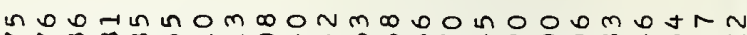
t

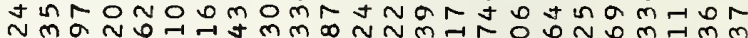

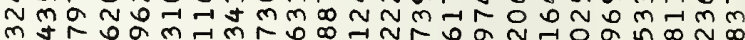

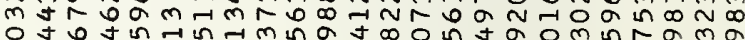
연

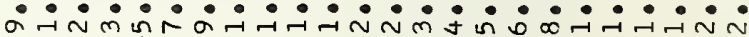

$\overrightarrow{1} \overrightarrow{1}$ ユન

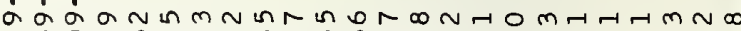

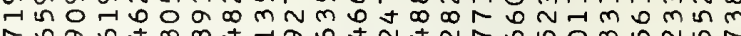

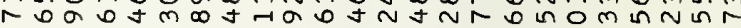
$\because \because \because 0 \Omega$

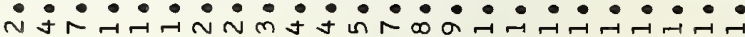

17 Н ન

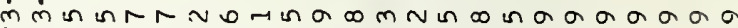

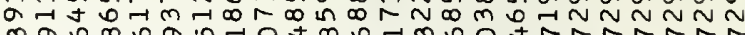

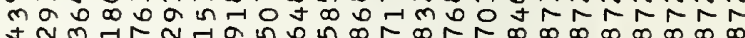

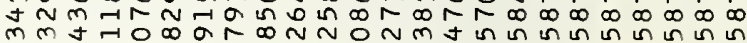

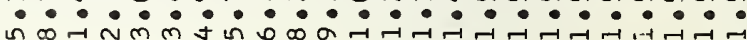

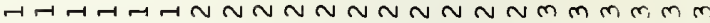

min 0 NO N

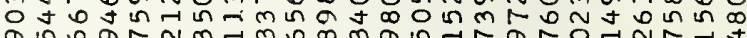

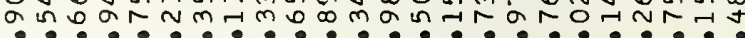

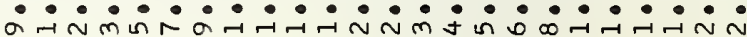
नન 1

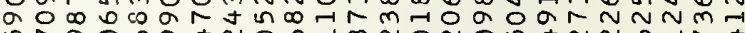

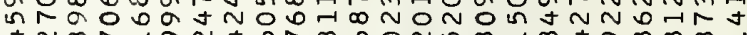

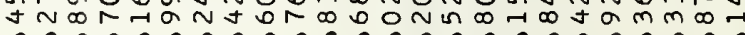
in

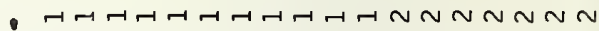

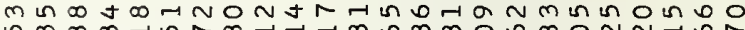

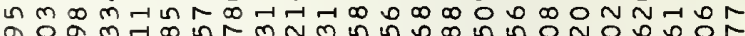

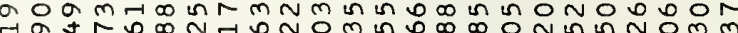

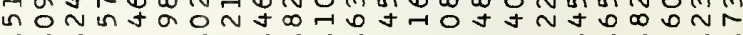

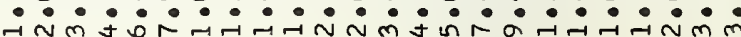

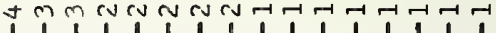
भ

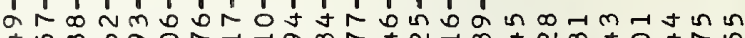
ง

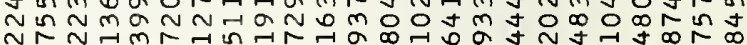

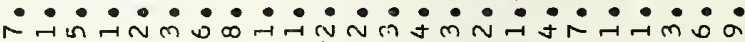
1,111111111111111

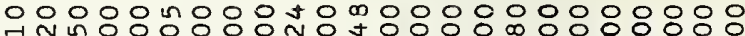

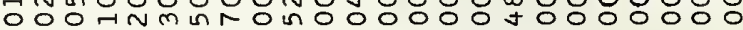

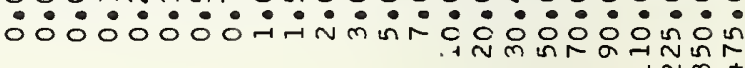

in in in in in in in in in in in in in in in in in in in in in in in in

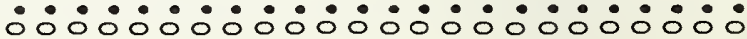

in in in in in in un in in in in in in in in in in in in un in in in in

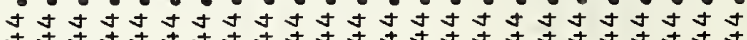
m mmmmmmmm 
mmm NNNNN

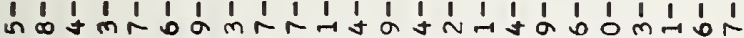

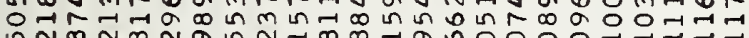

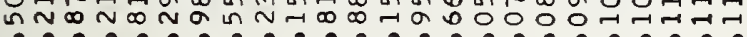

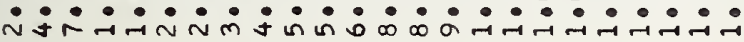

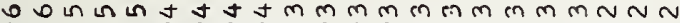

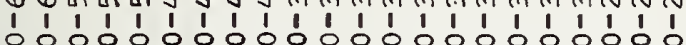

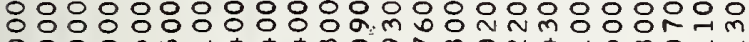
0000 in

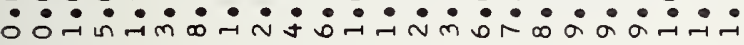

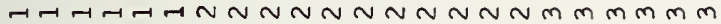

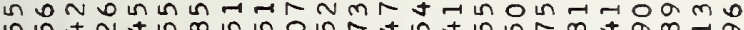
nim $m$ ba

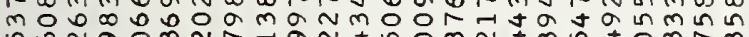
ñNaำ N Nammiona

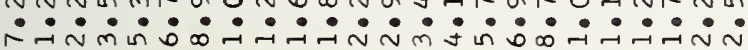

$\overrightarrow{1} \overrightarrow{1}$

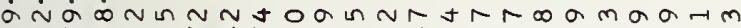

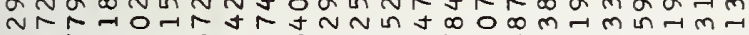

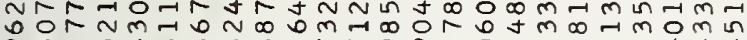

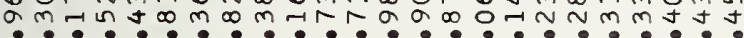

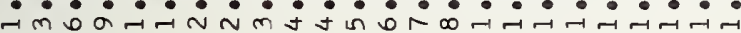

$7-1$

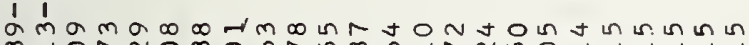

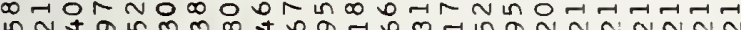

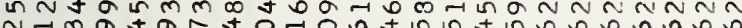
N $? \div ? \infty \infty$ in 6 in

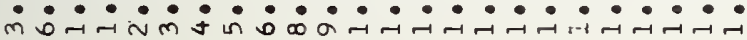

$\neg-\neg-7-1 \sim N \sim N \sim N \sim N \sim N m m m m$

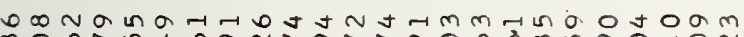
movring 0 a n

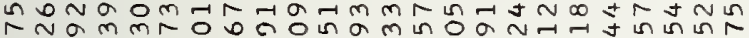
กำ in mi a o

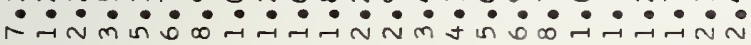

-

$\rightarrow \pi$

$\neg \neg \sqcap \neg \neg-\neg \neg$

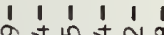

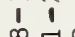

1 vin

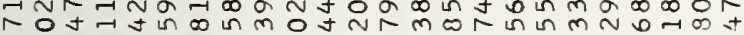
$0 \infty m-1$ in $m \rightarrow m$ in $r N b$ in 0 in $\infty \rightarrow \infty$ t $a m m \infty r$

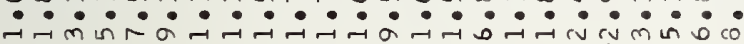
$\neg-\neg-1-1-1-1 \sim \sim \sim N \sim N \sim$

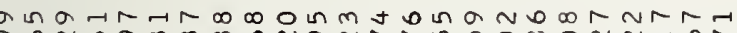

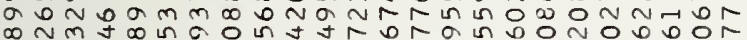

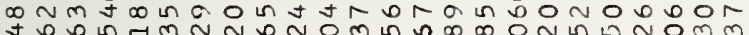

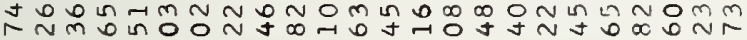

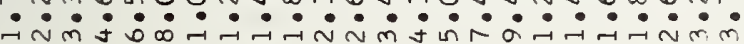

A $m m n \pi$

નศનન

$\begin{array}{lllllllllllllllll}1 & 1 & 1 & 1 & 1 & 1 & 1 & 1 & 1 & 1 & 1 & 1 & 1 & 1 & 1 & 1\end{array}$

o.

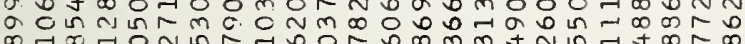

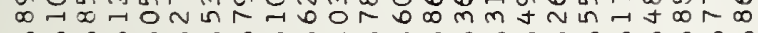

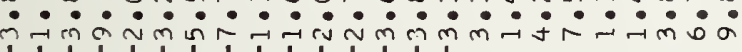

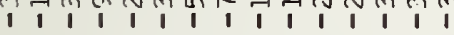

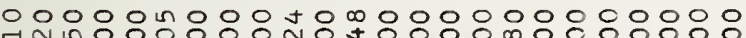

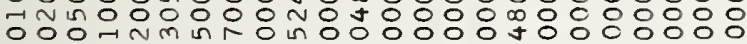

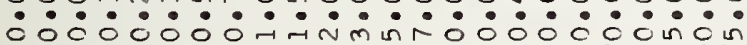

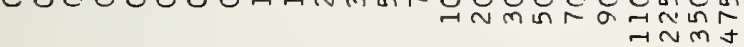

000000000000000000000000 -

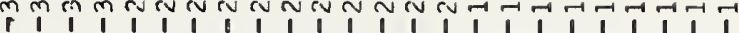

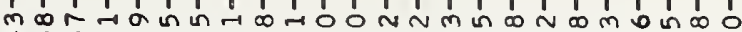

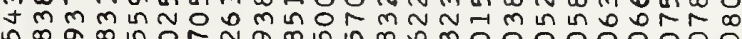

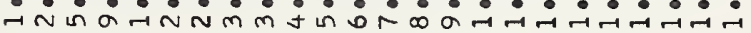

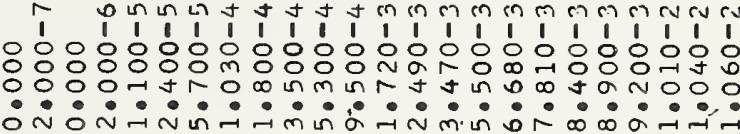

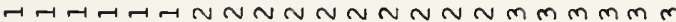

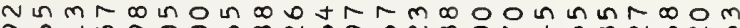

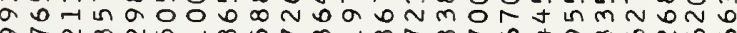

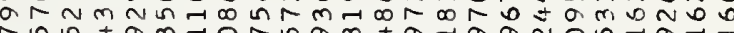
ก

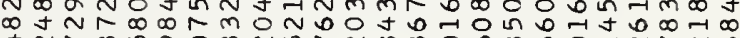

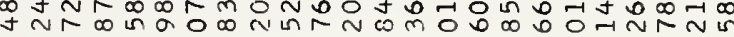

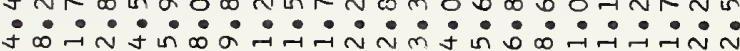

$\rightarrow-1 ㄱ ㅓ 1$

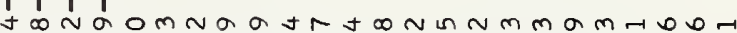

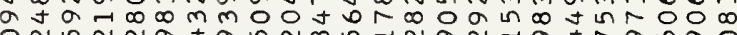

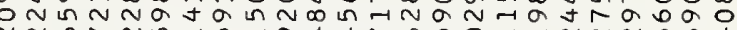

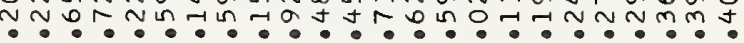

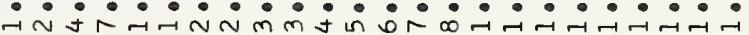

구

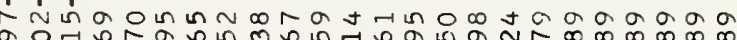

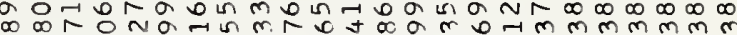
क ษ

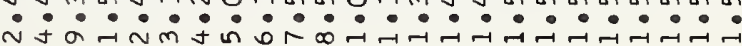

$-1-1-1-1+N \sim \sim \sim \sim \sim \sim \sim \sim \sim m m m m m$

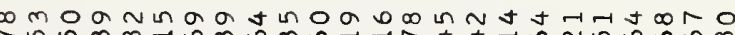

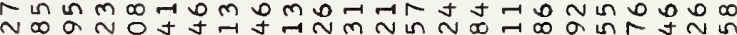

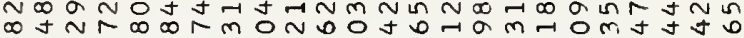

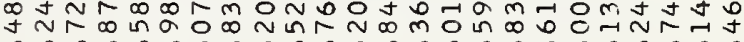

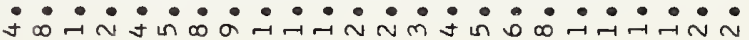

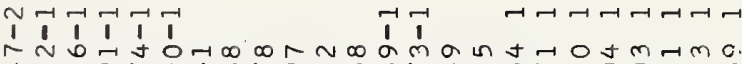

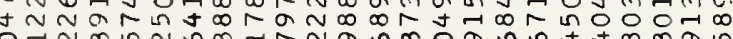

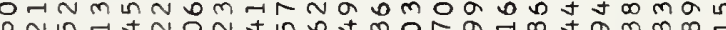

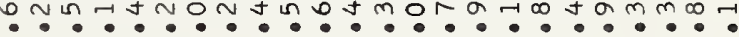

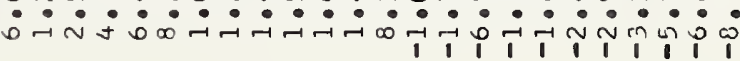

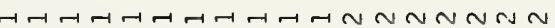

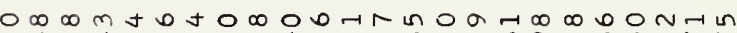

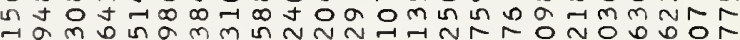

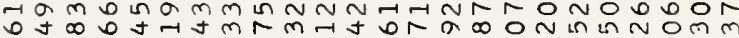

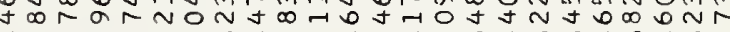

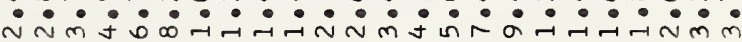

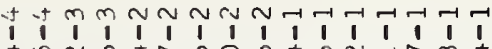

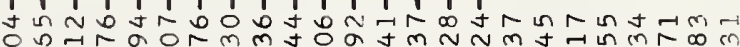
D

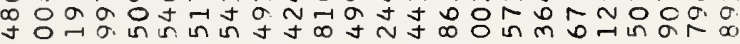

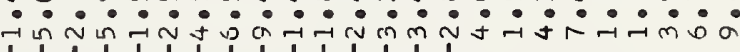

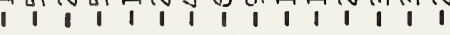

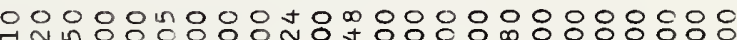

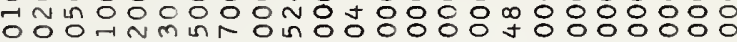

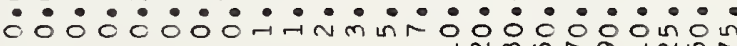
$\rightarrow$ N

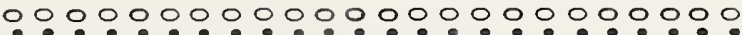

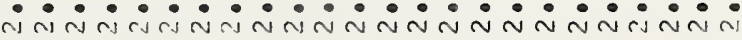


$\begin{array}{llllllllllllllllllllllllll}1 & 1 & 1 & 1 & 1 & 1 & 1 & 1 & 1 & 1 & 1 & 1 & 1 & 1 & 1 & 1 & 1 & 1 & 1 & 1 & 1 & 1 & 1 & 1\end{array}$ זw j

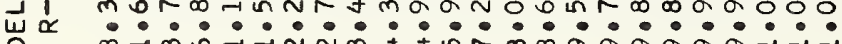

$\underset{⿱ ⺌}{\mathbb{L}}$

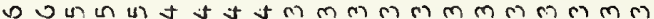

$\begin{array}{lllllllllllllllllllll}0 & 1 & 1 & 1 & 1 & 1 & 1 & 1 & 1 & 1 & 1 & 1 & 1 & 1 & 1 & 1 & 1 & 1 & 1 & 1 & 1\end{array}$ t

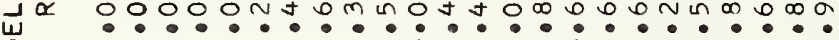

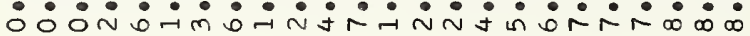

$\neg-\neg-\neg-\sim \sim N \sim \sim N \sim N \sim N \sim m m m m m$

ñ 0 잉

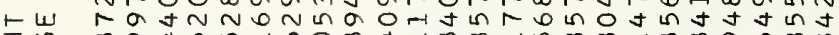

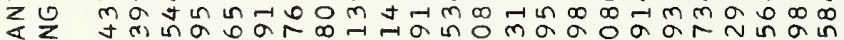
J证 जा

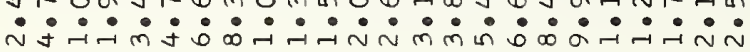

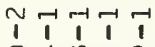

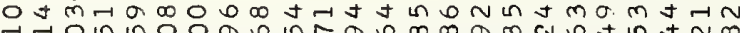
告出 品

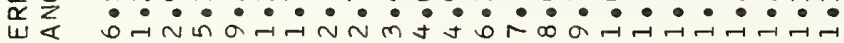

$\overrightarrow{1} \hat{1} \overrightarrow{1}$

每

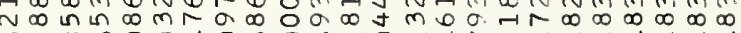
$\supsetneq$

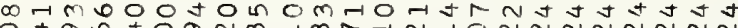

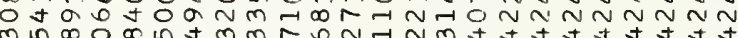
m in $\infty: 0$, m n

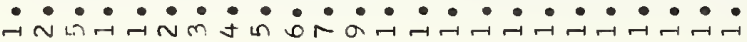

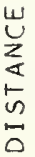

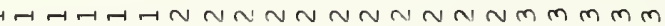

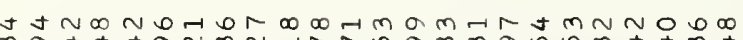
子

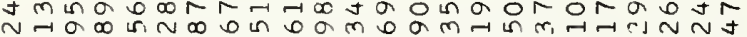
ษ

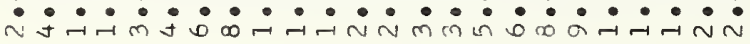

$\ll \ll \hat{1}$

11

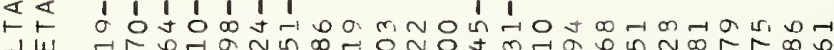
UI DF in $\sigma$ in

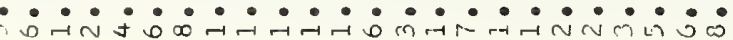

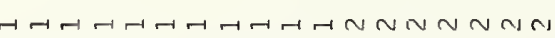

$\stackrel{\leftarrow}{\leftarrow}$

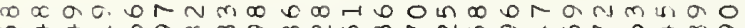
每

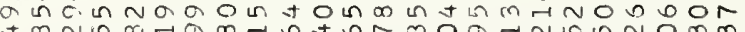

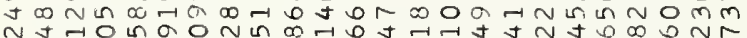

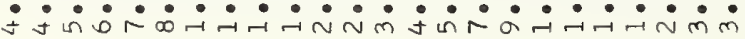

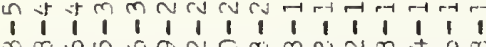

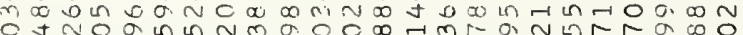
J vo an $n$ n

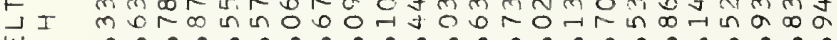

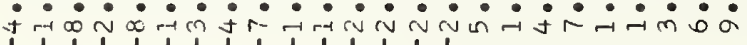

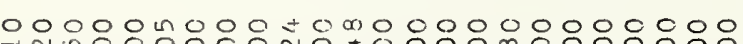
odn

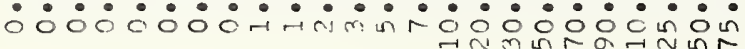
$\rightarrow \sim m y$

$\stackrel{t}{z}$

000000000000000000000000

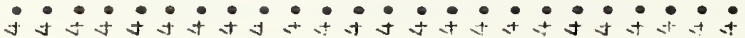

I

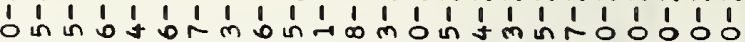

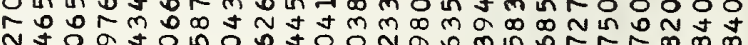

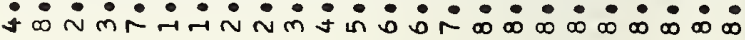

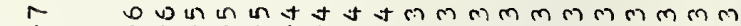
$\begin{array}{lllllllllllllllllllllllllllll} & 1 & 0 & 0 & 0 & 1 & 1 & 1 & 1 & 1 & 1 & 1 & 1 & 1 & 1 & 1 & 1 & 1 & 1 & 1 & 1 & 1 & 1 & 1 & 1\end{array}$

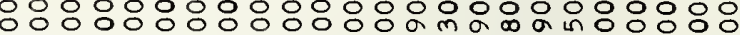

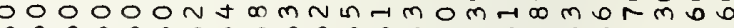

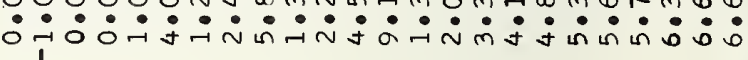

H- $-1-N N N N$ N

N N 0 H

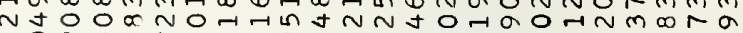

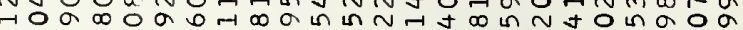
O

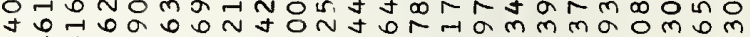
N

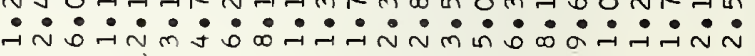

NTH

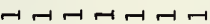

I I I I 1 in

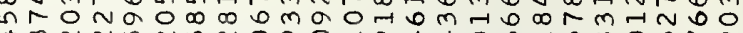

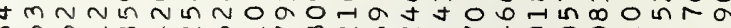
m?

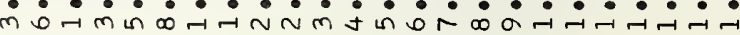

N $17-1$

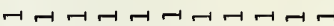

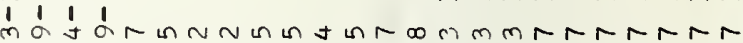

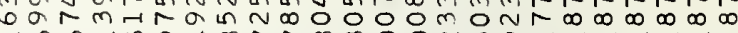
न

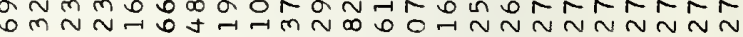
:-mininm

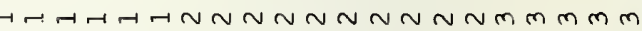

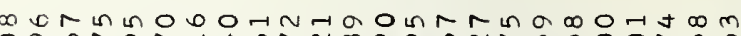

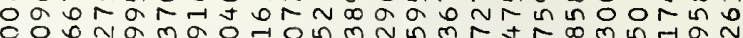

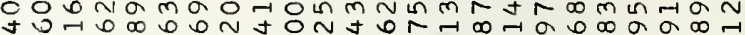
オ

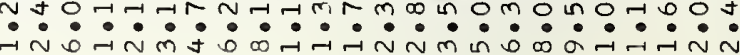

NN

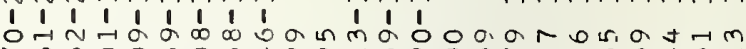

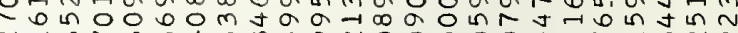

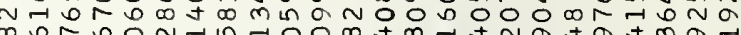
$\infty$ or 0 o

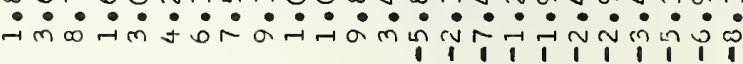

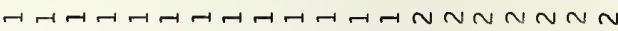

0 In ○.t

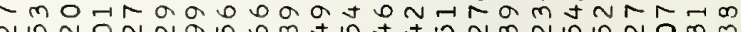

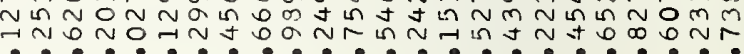

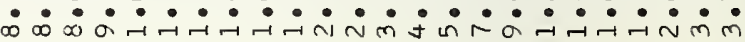

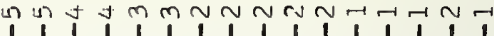
$\sqcap \sqcap \sqcap \sqcap$

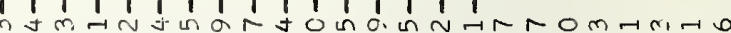

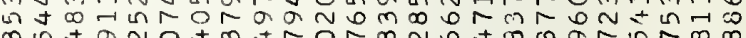

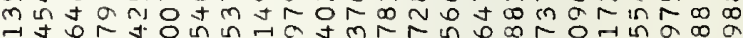

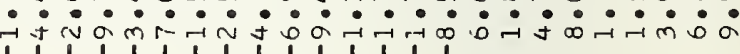

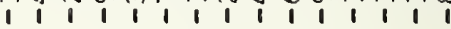

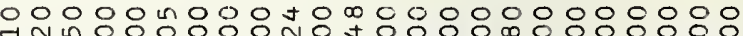

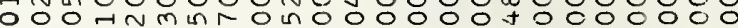

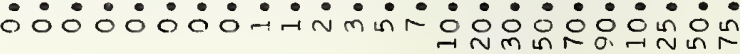
$\rightarrow \sim m+$

000000000000000000000000

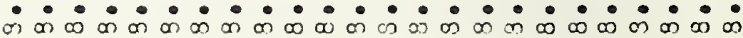




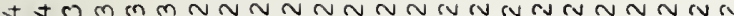

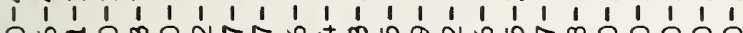

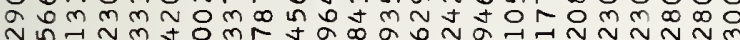

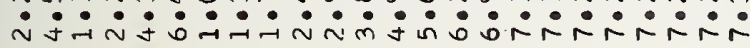

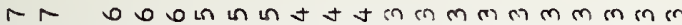
« o 낭ㅇㅇㅇㅇㅇㅇㅇㅇㅇㅇㅇㅇㅇㅇㅇㅇㅇㅇㅇㅇㅇㅇㅇㅇㅇㅇㅇㅇㅇㅇㅇㅇㅇㅇㅇㅇㅇㅇㅇㅇㅇ응

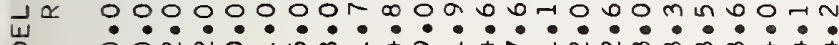
崩

1

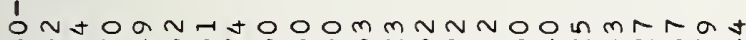
-

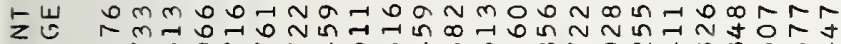

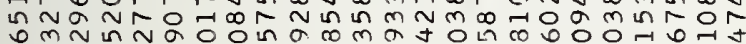

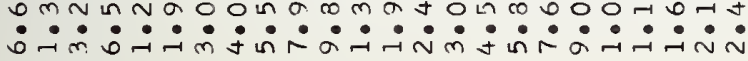

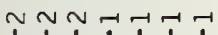

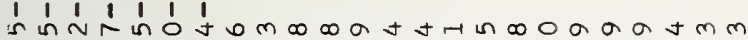
t $\alpha$ in 0 in $N$ in in in $t N m-0 m-10-N \sim O N$ in $N$

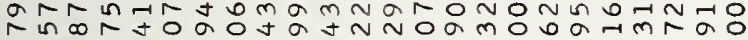

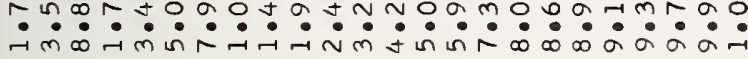

$\sin 1-1$

H-

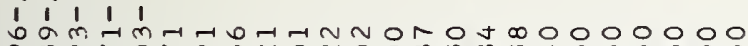

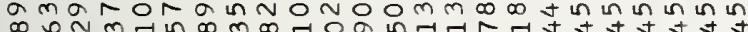

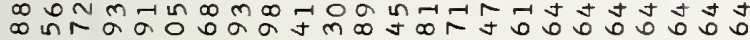

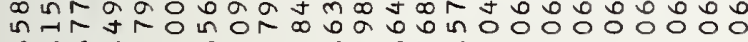

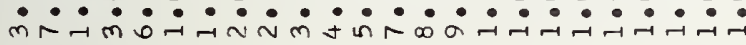
1.

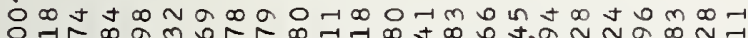

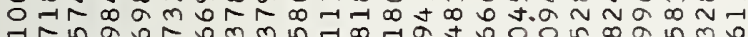

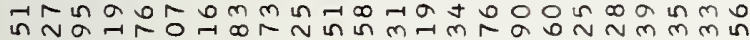

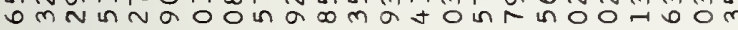

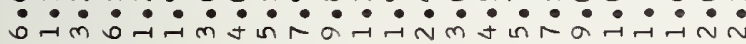

\&

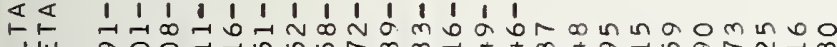

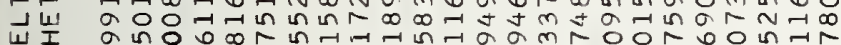
峁吉

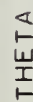

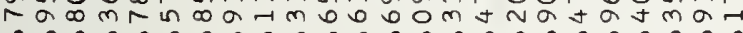

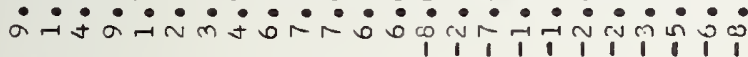

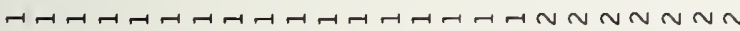

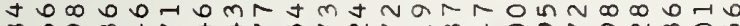

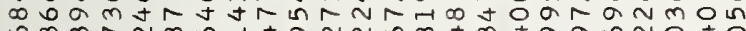

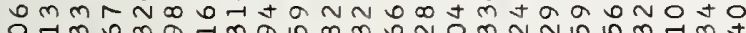

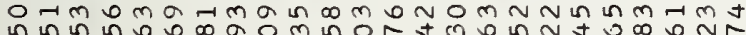

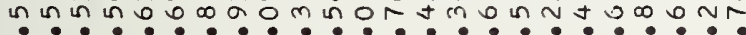

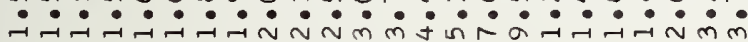

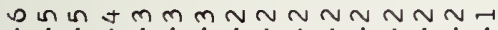

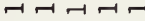

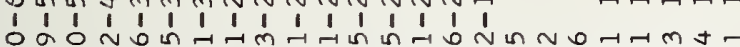
\& $\quad \infty$ in

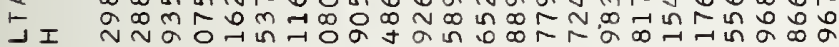

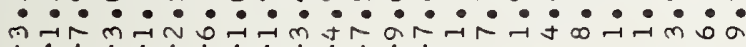
$\begin{array}{lllllllllllllll}1 & 1 & 1 & 1 & 1 & 1 & 1 & 1 & 1 & 1 & 1 & 1 & 1 & 1\end{array}$

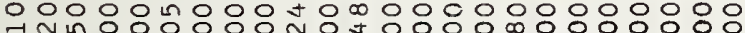

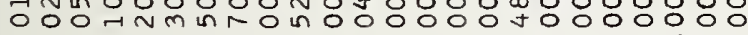

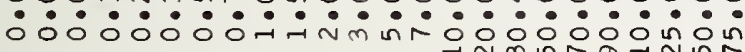

000000000000000000000000

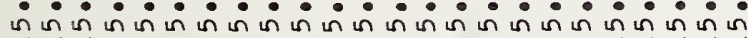

in in in in in in in in in in in in in in in in in in in in in in in in

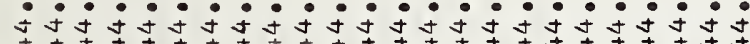

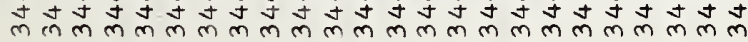

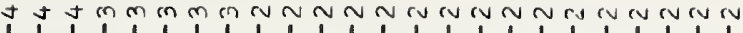

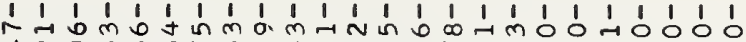

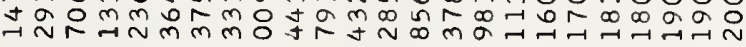

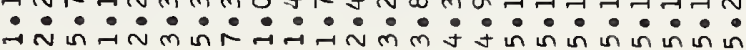

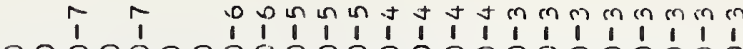

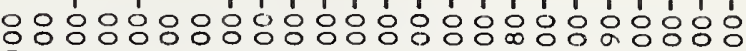

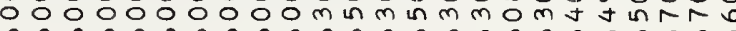

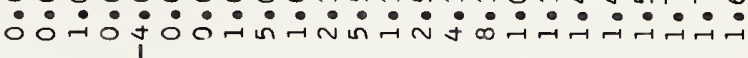

$\overparen{1}$

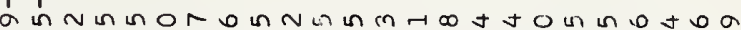
ง นn

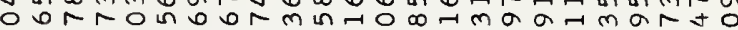

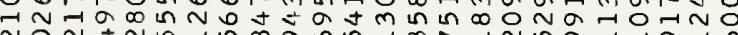

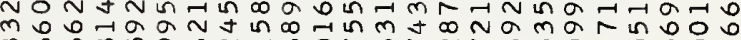

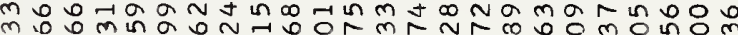

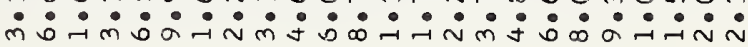

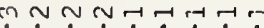

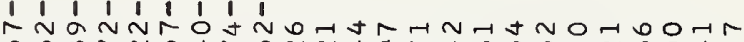

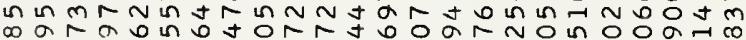

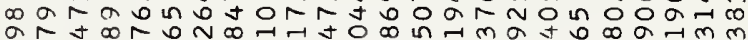

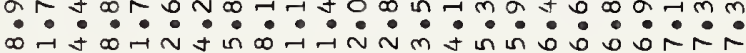

$\operatorname{1n} 2 \pi-1-1$

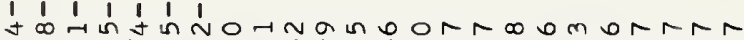

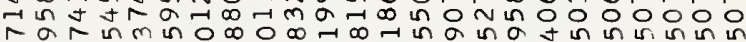


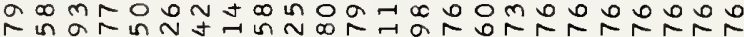

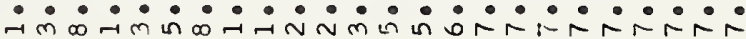

11

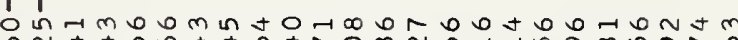

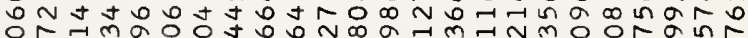
m in

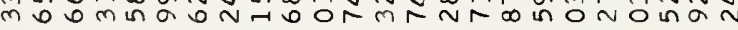

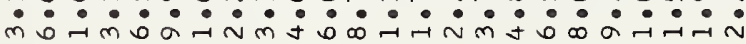

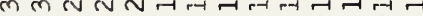

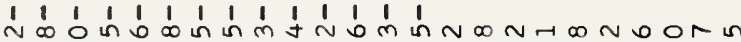

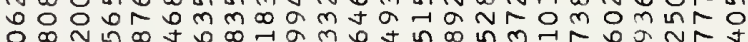

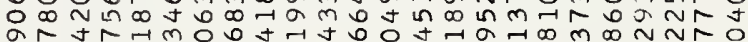

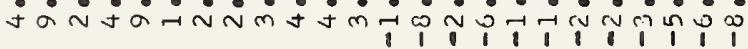

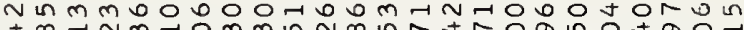

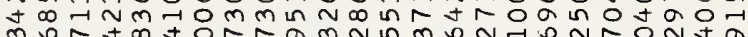
món mojóm

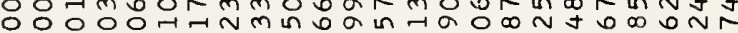
$\dot{m} \dot{m} \dot{m} \dot{m} \dot{m} \dot{m} \dot{m} \dot{m} \dot{m} \dot{j} \dot{i} \dot{i} \dot{\infty} \dot{a} \dot{-} \dot{-} \dot{i} \dot{\sim} \dot{m} \dot{m}$

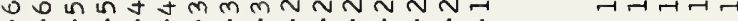

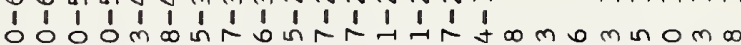
n moora JT N N

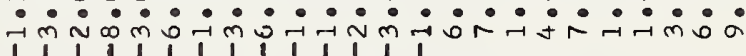

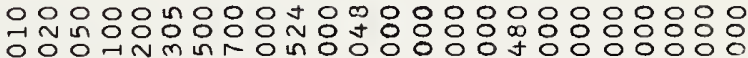
$\dot{0} \dot{0} \dot{0} \dot{0} \dot{0} \dot{0} \dot{i} \dot{\sim} \dot{\mathrm{i}} \dot{0} \dot{0} \dot{0} \dot{0} \dot{0} \dot{0} \dot{0} \dot{0} \dot{0}$ ำ

000000000000000000000000

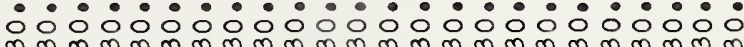

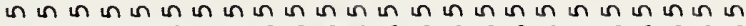

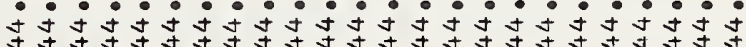
m m m m 


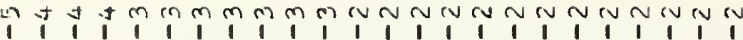

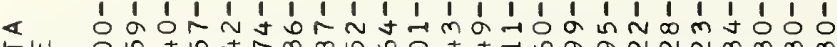
出

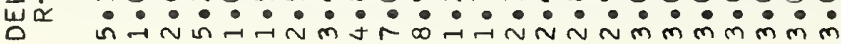

$\varangle$ $\perp$ 또 w

$\sum_{<}^{1}$

ज) $\frac{1}{\alpha}$

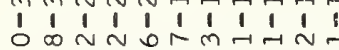

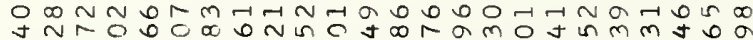

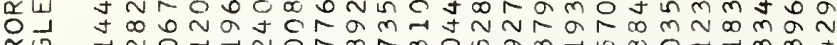

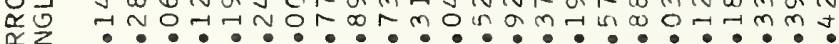

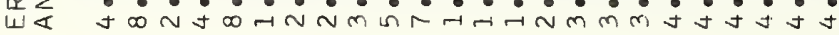

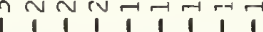

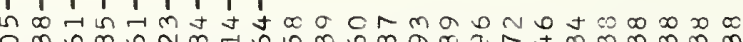

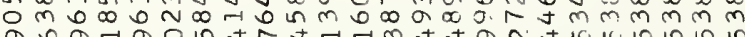
$\underset{4}{2}$

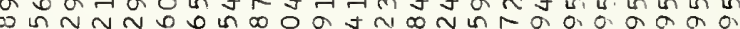
$\sim b r N b y a+i n m a r N \infty$ in in in in in in in in

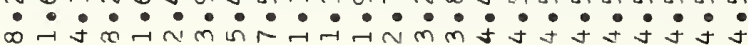

$\overrightarrow{1}+\overrightarrow{1}$

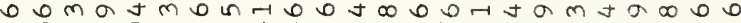

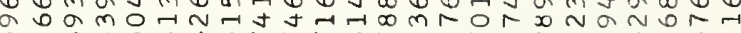

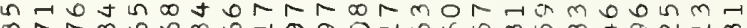

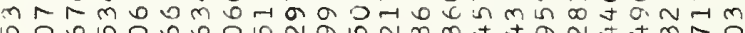

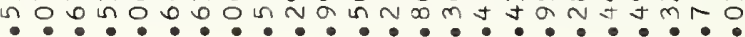

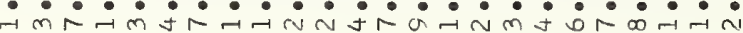

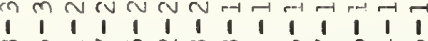

$\ll<$

步㞬

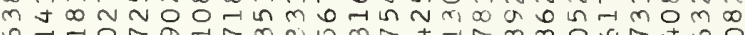
ข

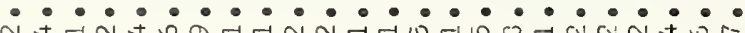
111111111111

里

I

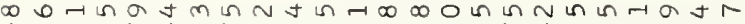

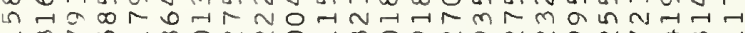

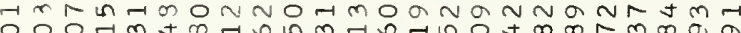

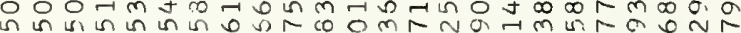

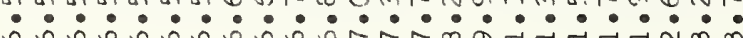

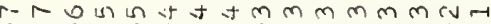

$\begin{array}{llllllllllllllllllllll}1 & 1 & 1 & 1 & 1 & 1 & 1 & 1 & 1 & 1 & 1 & 1 & 1 & 1 & 1 & 1\end{array}$

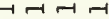

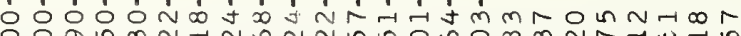

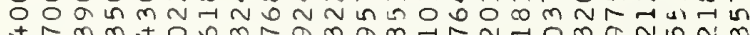

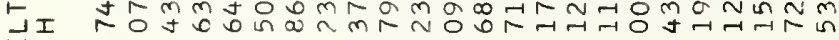
WI

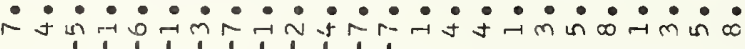
$1,1,1,1,1,1$

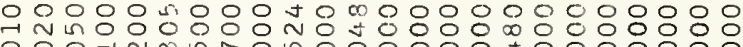

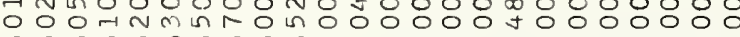
$\dot{0} \dot{0} \dot{0} \dot{0} \dot{0} \dot{0} \dot{\sim} \dot{\sim} \dot{0} \dot{0} \dot{0} \dot{0} \dot{0} \dot{0} \dot{0} \dot{0}$ rin

Im

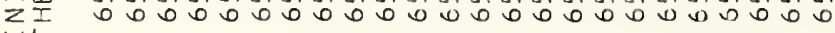

w

in in in in in in un in in in in in:

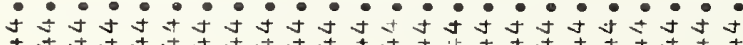
mं

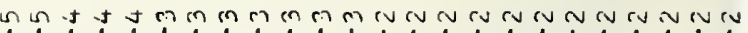

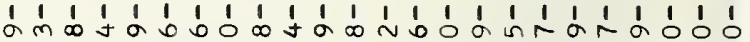

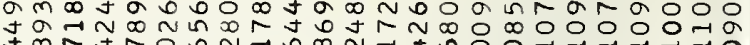

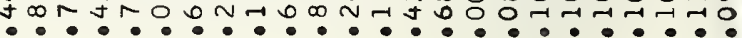
$\dot{m} \dot{0} \dot{m} \dot{0} \dot{\sim} \dot{\sim} \dot{m} \dot{j} \dot{n} \dot{\infty} \dot{\sim} \dot{\sim} \dot{\sim} \dot{\sim} \dot{\sim} \dot{\sim} \dot{\sim} \dot{\sim} \dot{v} \dot{v}$

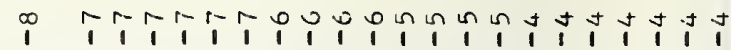

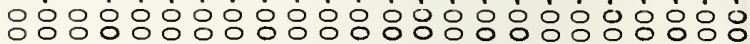
0000000000000100000

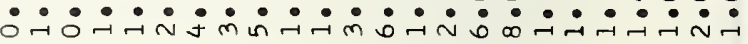

$\stackrel{1}{1}$

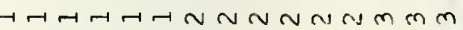

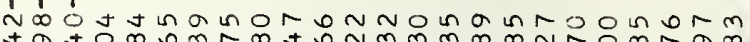
ta t $000 m+\infty$ t $0 N m m m \infty \infty N T 0 \infty N a \infty$

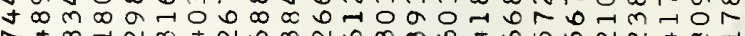

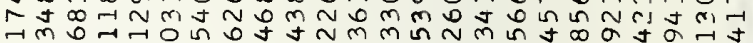

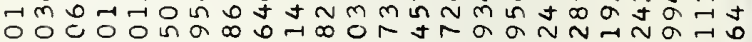
ㅇㅇㅇㅇㅇㅇㅇㅖ

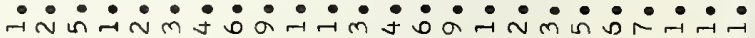

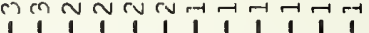

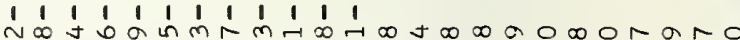

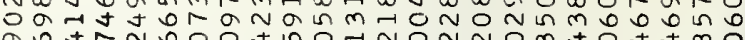

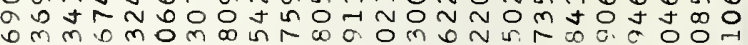

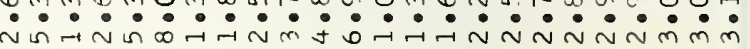

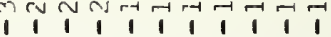

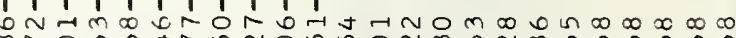

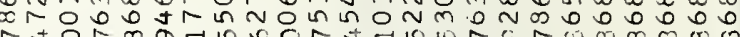
-

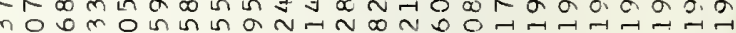

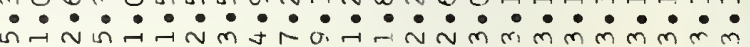

$\widetilde{1}$

$\rightarrow-1-1 \rightarrow N$ N $\rightarrow$ N

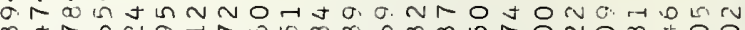

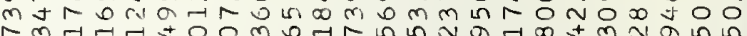

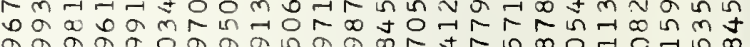

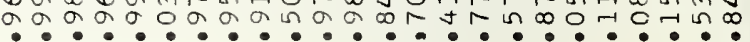

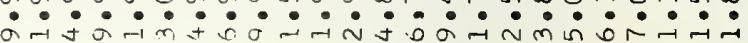

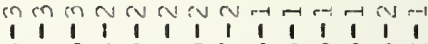
$\sim r-\sqcap-\sqcap-r$

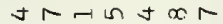

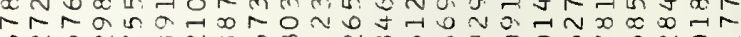

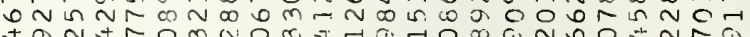
ง

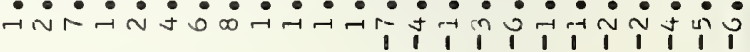

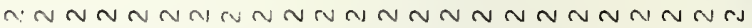

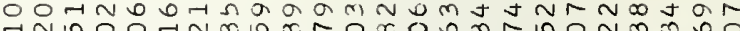

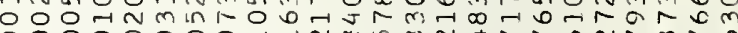

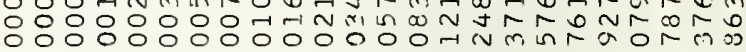

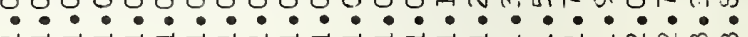

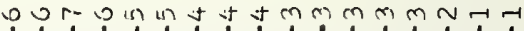

$-1-1-1$

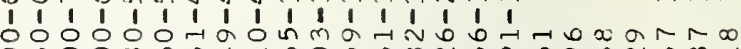

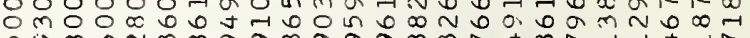

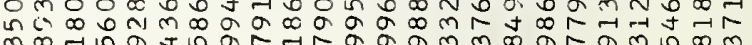

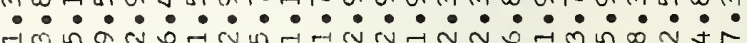
$\begin{array}{lllllllllll}1 & 1 & 1 & 1 & 1 & 1 & 1 & 1 & 1\end{array}$

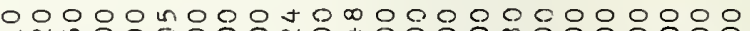

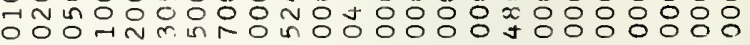

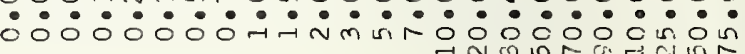
$\rightarrow \sim m y$

000000000000000000000000 ○ $\dot{0} \dot{0} \dot{0} \dot{0} \dot{0} \dot{0} \dot{0} \dot{0} \dot{0} \dot{0} \dot{0} \dot{0} \dot{0} \dot{0} \dot{0} \dot{0} \dot{0} \dot{0} \dot{0}$

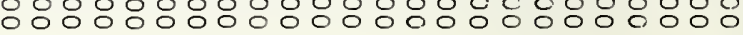

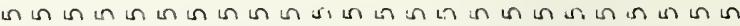

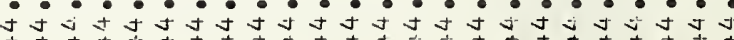

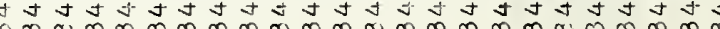




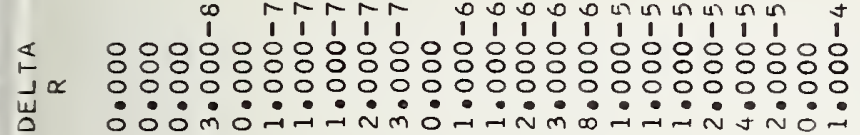

$1 \overrightarrow{1} \overrightarrow{1} \overrightarrow{1}$

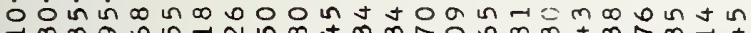
ŁU

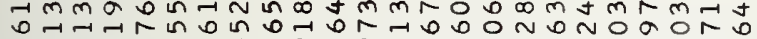

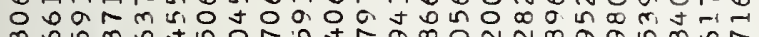

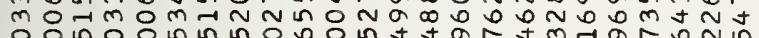

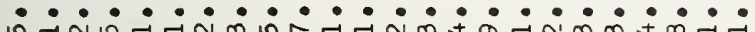

mmm N

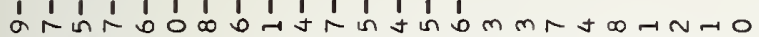

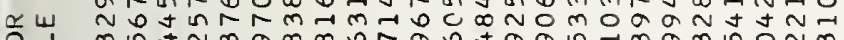
m

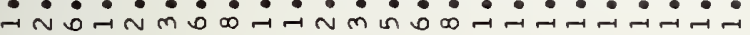

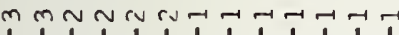

$\begin{array}{lllllllllllll}1 & 1 & 1 & 1 & 1 & 1 & 1 & 1 & 1 & 1 & 1 & 1 & 1\end{array}$ ă

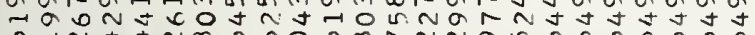
orntand

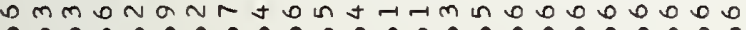

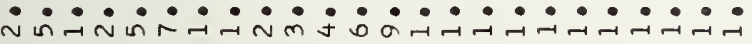

$\operatorname{in\rightarrow -1} \rightarrow-1$

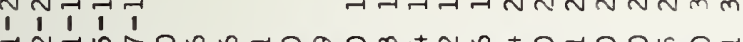

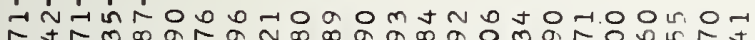
ñ

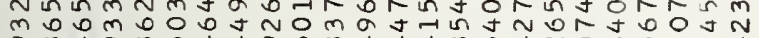

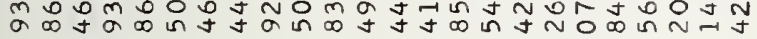

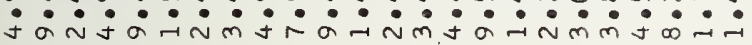

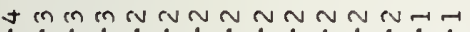

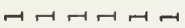

$<\varangle$

出

○下

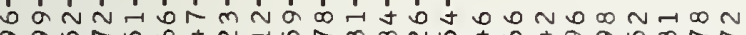
og a

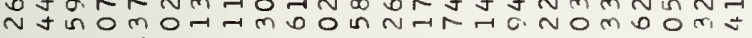

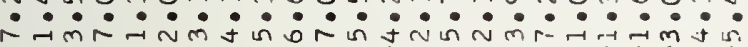

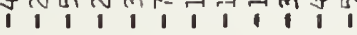

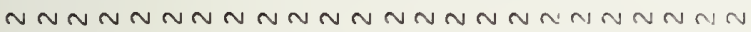

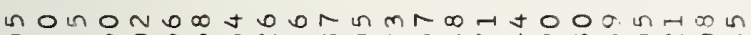

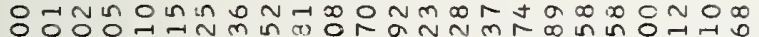

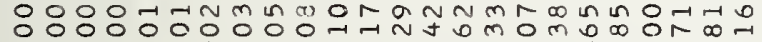

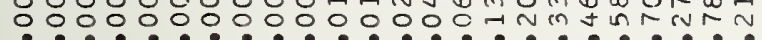
$\dot{\sim} \dot{\sim} \dot{\sim} \dot{\sim} \dot{\sim} \dot{\sim} \dot{v} \dot{v} \dot{\sim} \dot{\sim} \dot{\sim} \dot{\sim} \dot{\sim} \dot{\sim} \dot{\sim} \dot{\sim} \dot{\sim} \dot{\sim} \dot{m} \dot{j} \dot{j}$

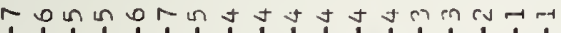

$\rightarrow H-1$

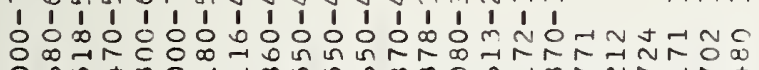

$\ll$

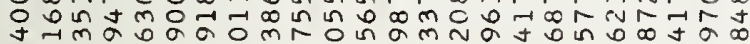

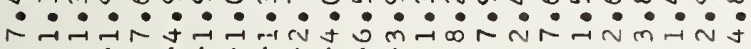

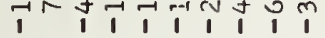

00000 mo $0070 m 000000000000$

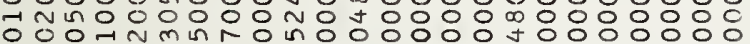

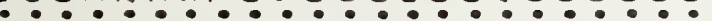

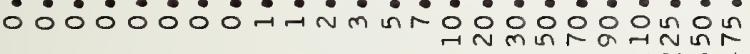
$\rightarrow \sim m$ t
000000000000000000000000

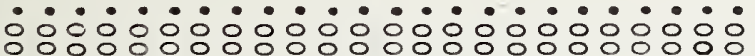

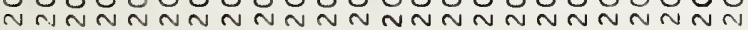

in แn

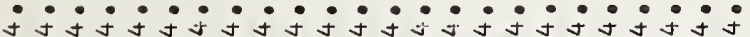

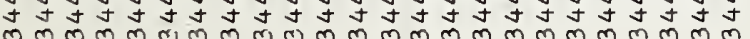

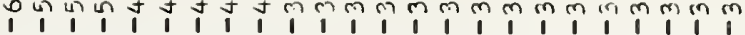

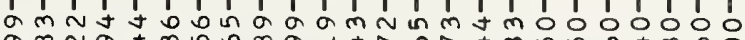

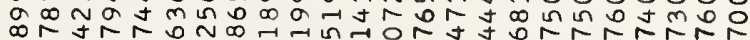

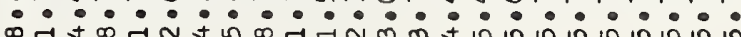

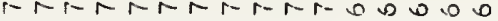

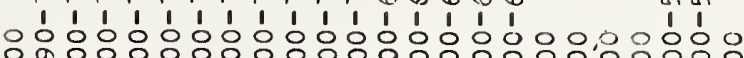

in in

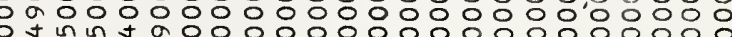
-

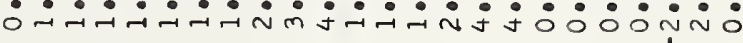

$\operatorname{1in}+1+1$

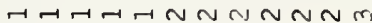

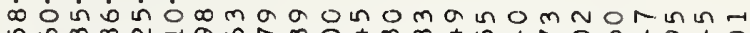

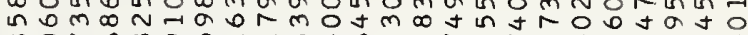

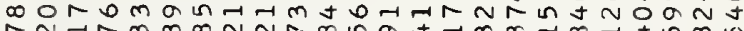

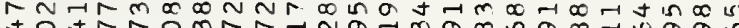

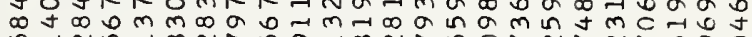
in

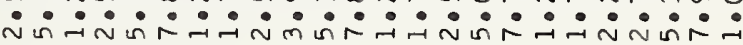

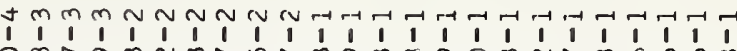

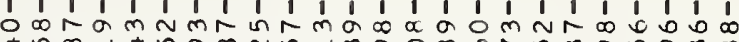

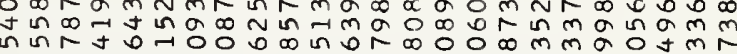

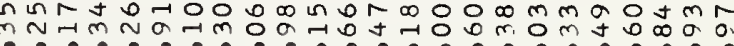

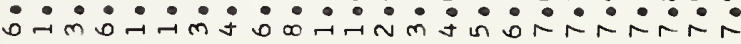

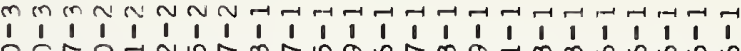

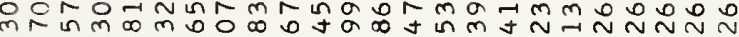
DO in $00 \pi$ t

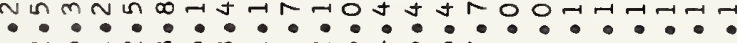

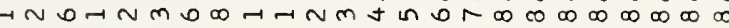

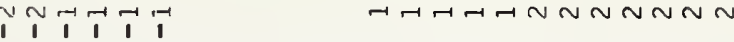
' I ' $m$ '

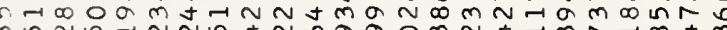

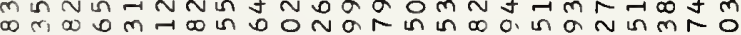

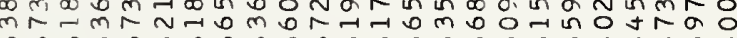

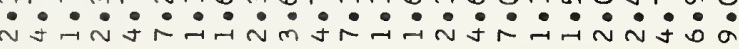

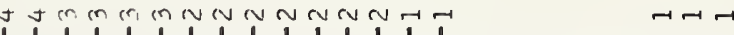
j

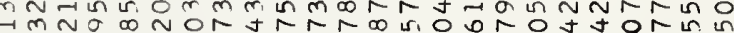

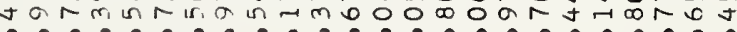

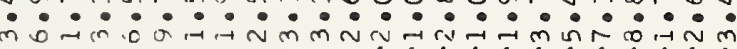

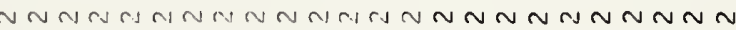

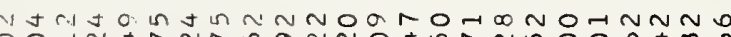

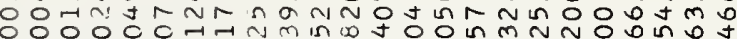

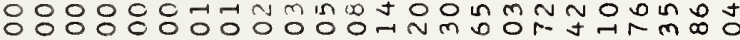
$0000000000000000 \pi n n m m 00$

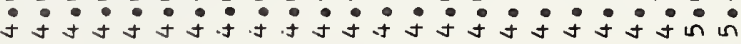

की पी

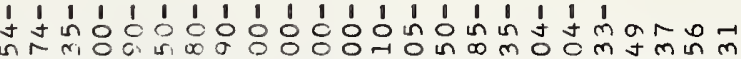
m

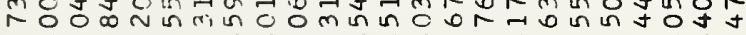

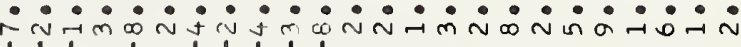
$\begin{array}{lllllll}1 & 1 & 1 & 0 & 0 & 0 & 0 \\ 1 & 1 & 1 & 0 & 0\end{array}$

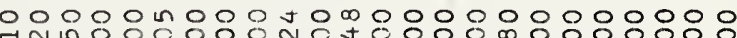

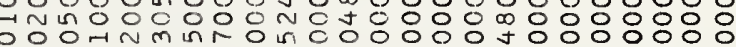

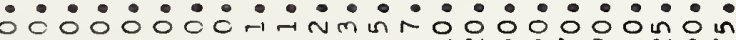
$\rightarrow$ N m in

000000000000000000000000

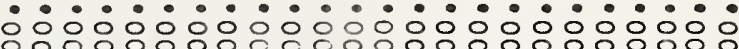

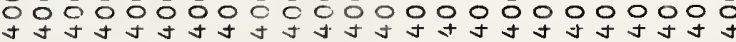

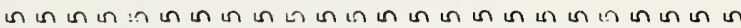

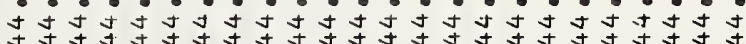

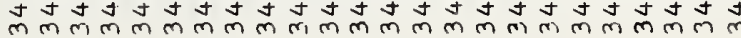


$\hat{1} \mathfrak{1} \hat{1} \mathfrak{1}$

$r m-4 \pi N N N$

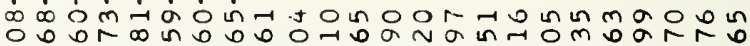

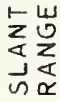

n mNarR DR

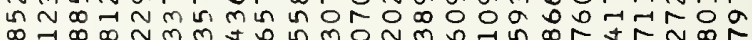

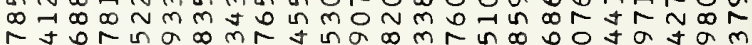

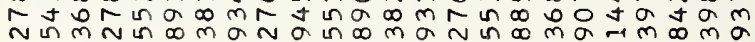

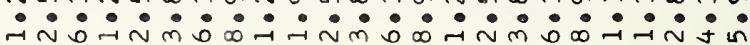

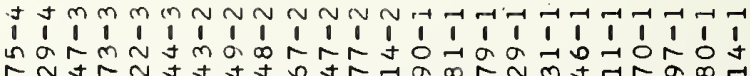

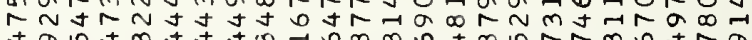

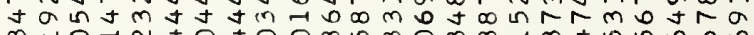

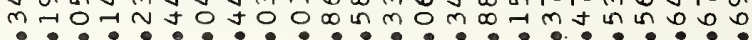

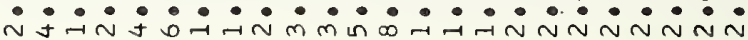

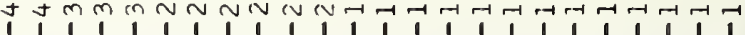

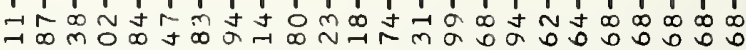

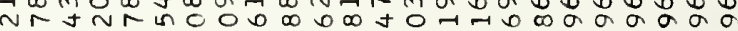

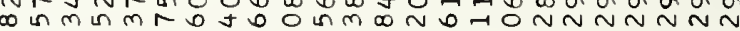

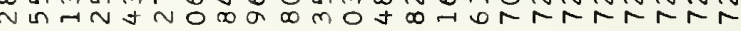

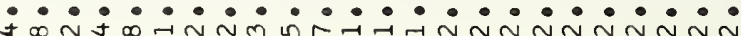

in $N$ N obagomrmon

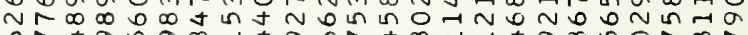
D T $\begin{aligned} & \infty \\ & 0\end{aligned}$

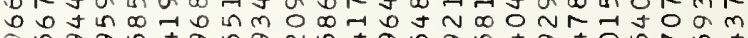

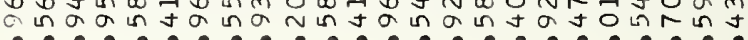

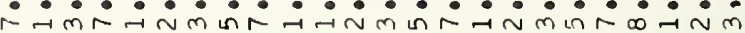

Atammmmm nnm

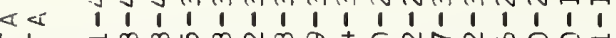

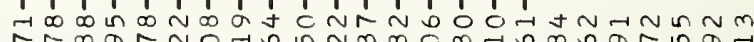

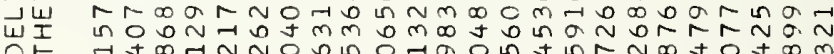
$\because \pm \infty r$ ก

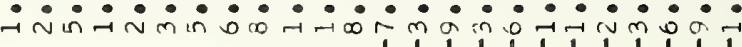
N $N \sim N \sim N \sim N \sim \sim N \sim N \sim N N \sim N \sim N N \sim N$ $\stackrel{\mathbb{I}}{\stackrel{\leftarrow}{ \pm}}$

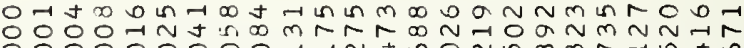
응ㅇㅇㅇㅇㅇ은

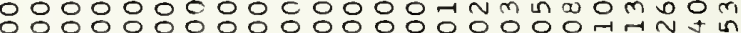
$\because 0 \% 00000000000000007 \pi$ ?

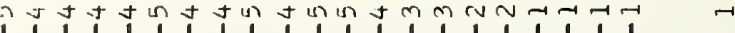

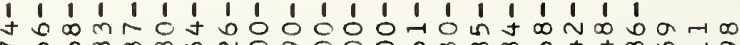
$\varangle$

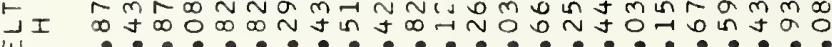
山

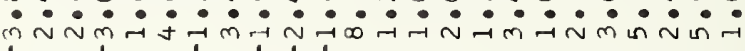

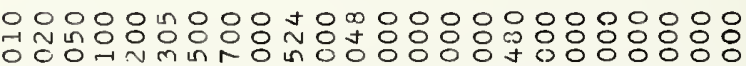
$\stackrel{1}{ \pm}$

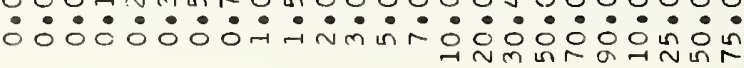

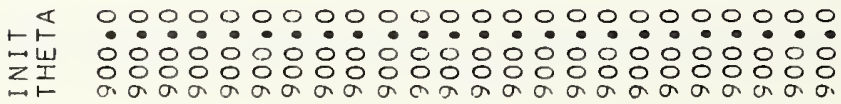


m mann

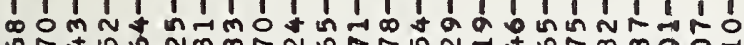

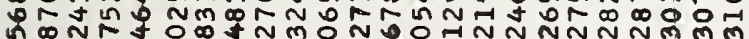

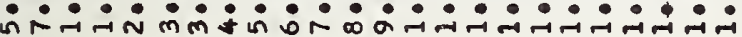

O

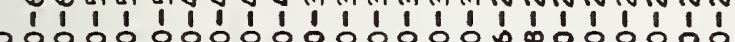

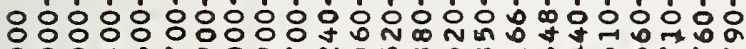

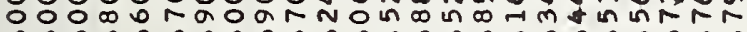

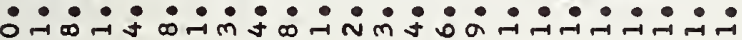

- $-1-H \rightarrow N N N N N N N N N N N N m m m m m m$

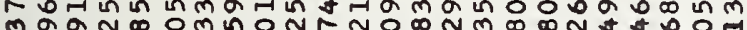

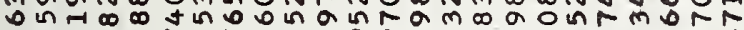

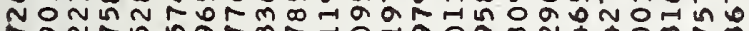
Nantm natm mo † 0 \%

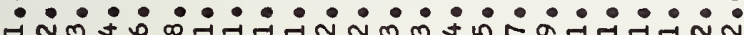

1.

- $\rightarrow$ - $\rightarrow$ - $\rightarrow$ - $\rightarrow-1$

T

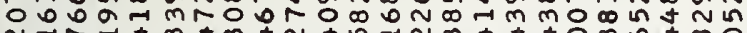

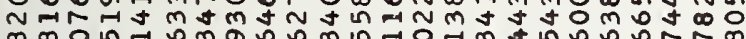

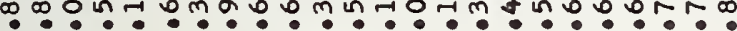

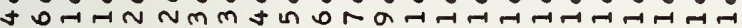

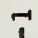

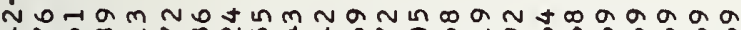
ษNษ

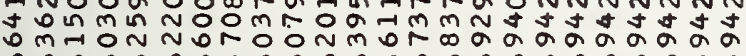
ăv

- $-7-7 N$ -

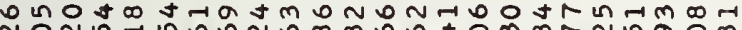
NON

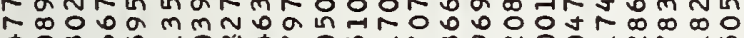
э

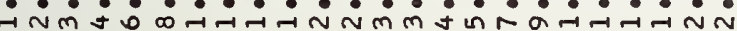

$\because \pi$

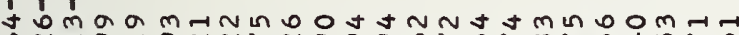

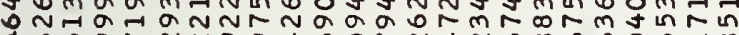

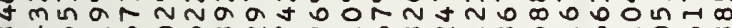

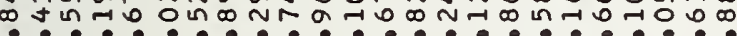
m

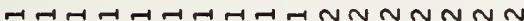

in $-N \sim N \infty$ in $N$ in $N a r j m$ in $m$ in $-1 \infty N \sim \infty 0$

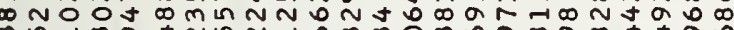

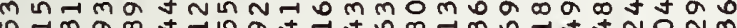
mă

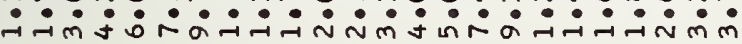

mmNnNNTHATH-T

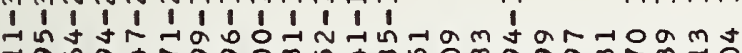
- 90 j w

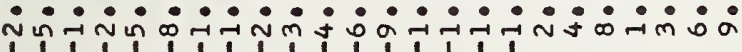

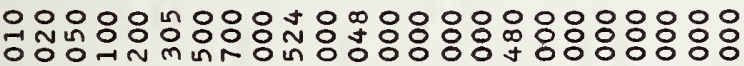

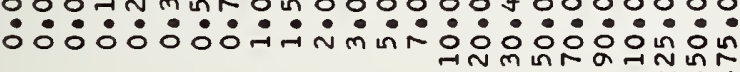

000000000000000000000000 ல்

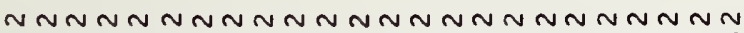

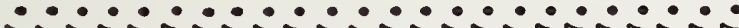
NANANANANNANANANANNANANA $\mathrm{mm} m \mathrm{~mm} m \mathrm{~mm} m \mathrm{~mm} m \mathrm{~mm} m \mathrm{~mm} m \mathrm{~m}$

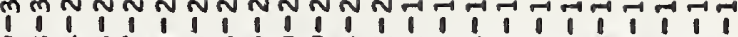

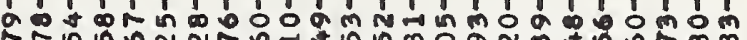
R

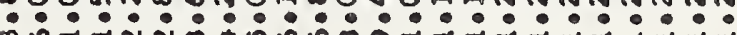
m OHAN

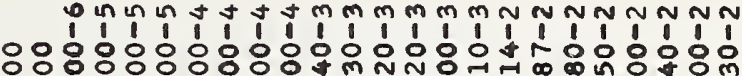

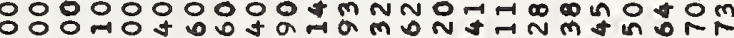

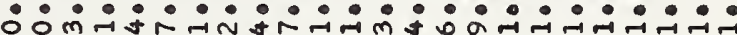

H-H $-1 \rightarrow N N$ N

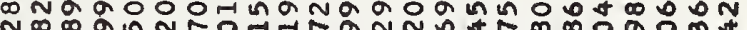
-

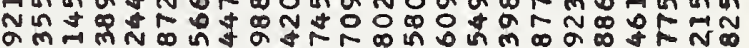
N $\rightarrow$ in 0 O 0 O -

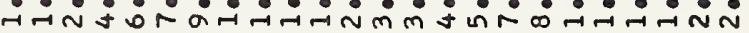

$\overparen{1} \mapsto$

-

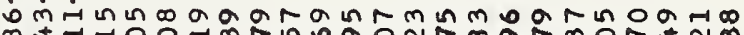

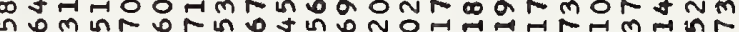

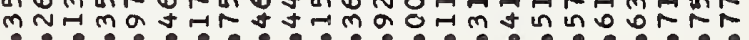

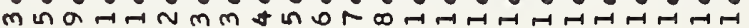

1

on

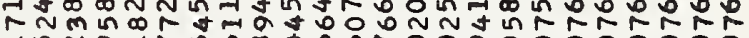

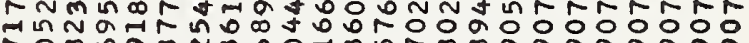

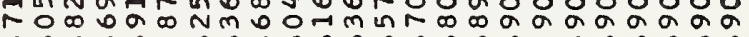

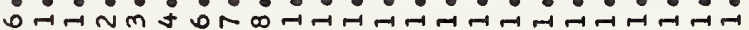

- $-1-7 \rightarrow N N$ N n N

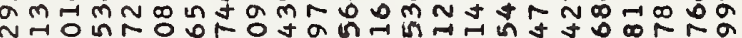

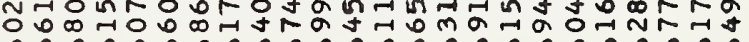

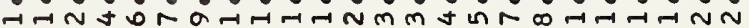

171

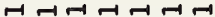

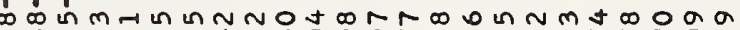

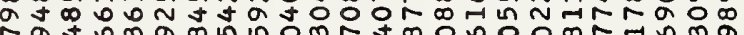
a t oma n m

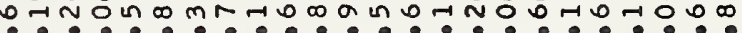
NオNーAーNNMmmm

-

mog

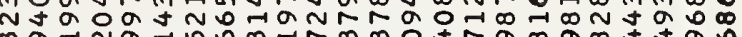
manNaำ ง

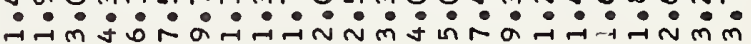

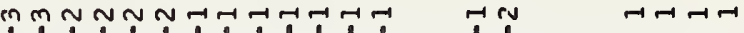
'́

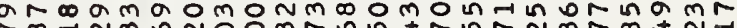
m

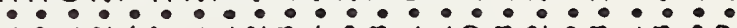
TM

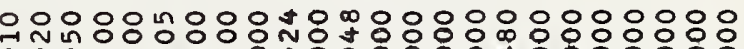

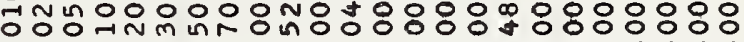

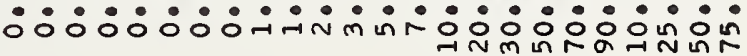

ก เก เก เก เก เก แก แก เก เก เก เก เก

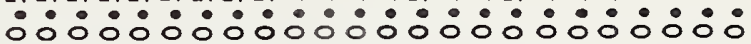

NNNNNNNNNNNNNNNNNNNNNNNN

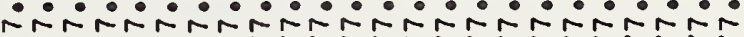

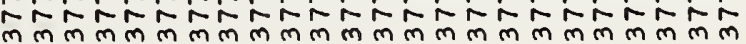



âd 1 岁匹

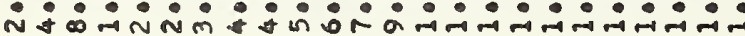

○ 0 m 4 t 00101010

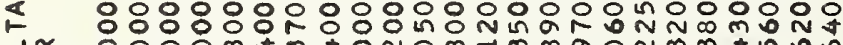
Ta 0000 t

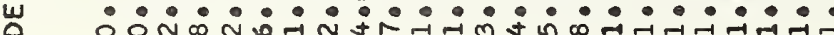

-1 -1 -1 N N N N N N N N

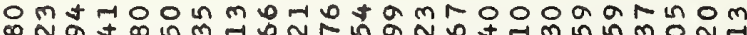

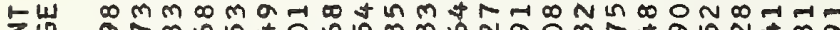

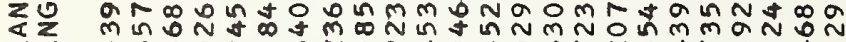

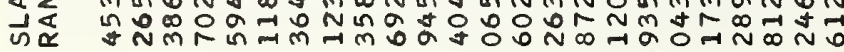

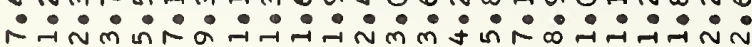

H1 ง mño-100

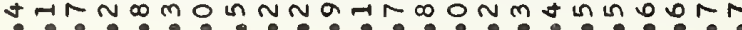

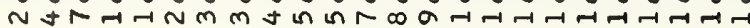

$\ddot{1}$

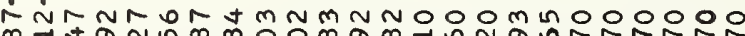

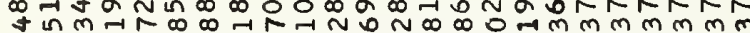
ปี

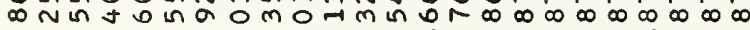

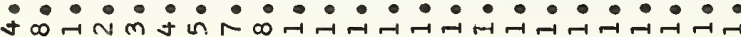

H-7-7-

$\frac{u}{4}$

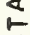

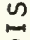

$\llbracket \llbracket$

嵌

- NNAmaatnmonombatonOORN

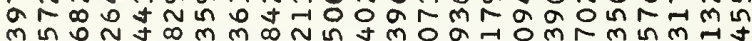

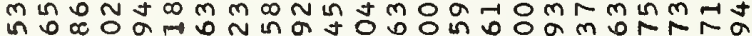

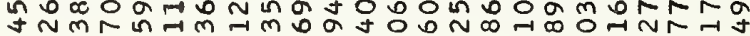

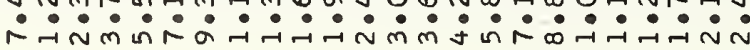

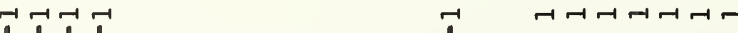
'ál'

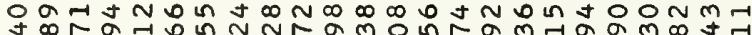

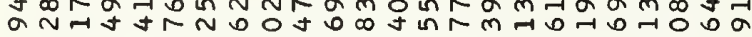

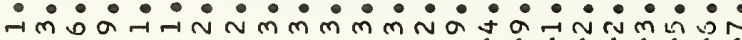

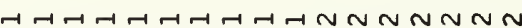

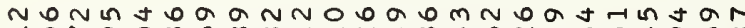
- mo-

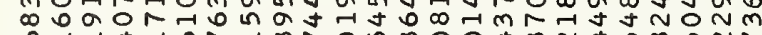
I $0-7$ t

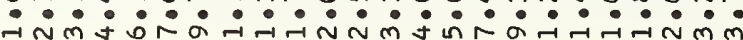

tmm N $m$ N ง

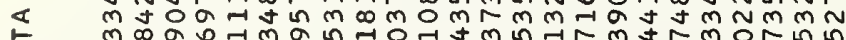

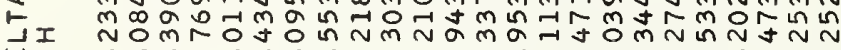

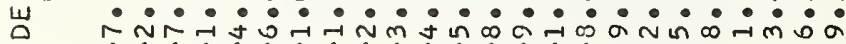

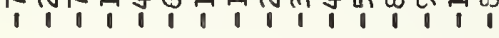

昰

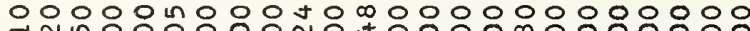

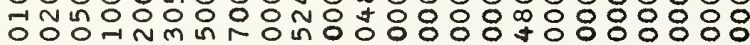

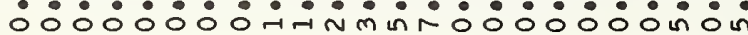

r =
U

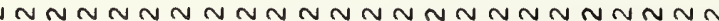

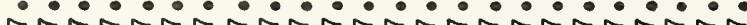

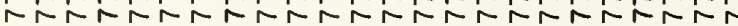

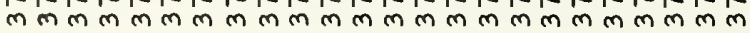
ำ

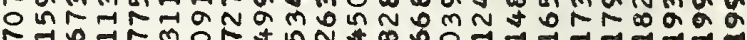

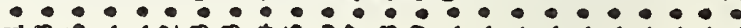
m

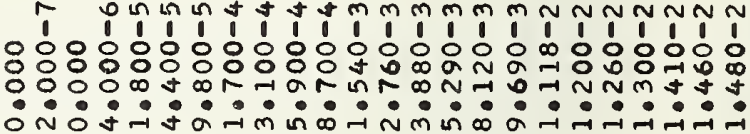

H-H-NNNNNNNNNNNmmmmmm

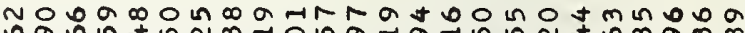

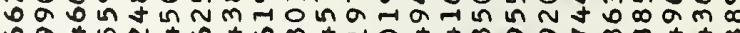

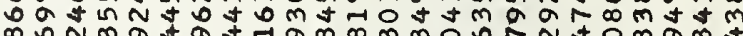
a N⿴囗十 N ?

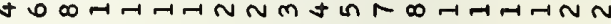

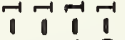

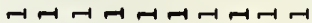

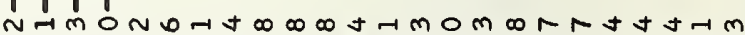

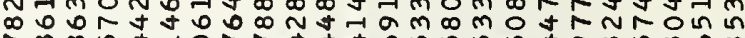

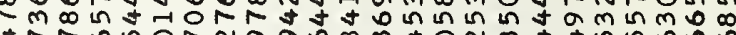
y T 0 แ

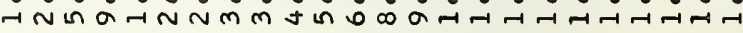

1 A

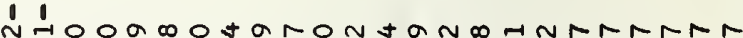
tor $m-7 a$ in

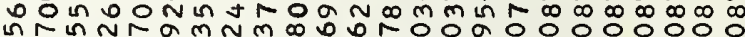

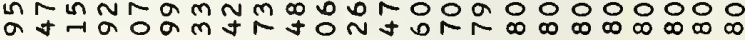

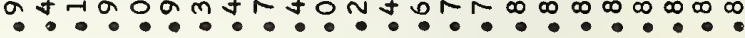

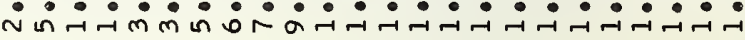

T-MHMNNNNNNNNNNMmMmMm

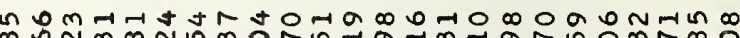

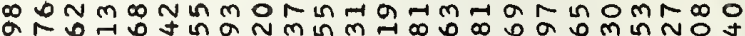
N กึ

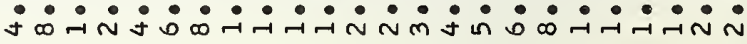

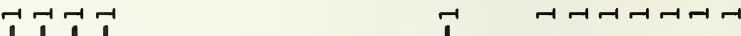
I'

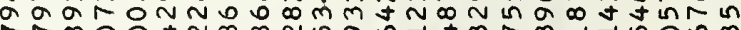

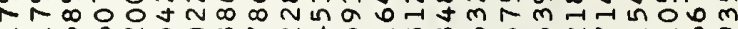
$\because \because$ ก

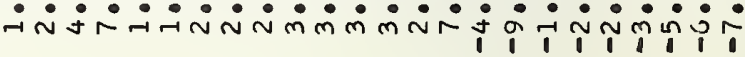

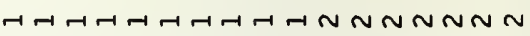

tNomoom nmortamntט जิ $\rightarrow 0 m m \circ 0-1$ o in $N$ in

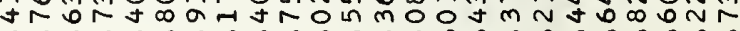

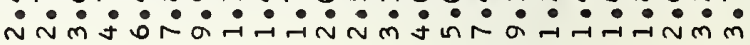

Aงm纤N

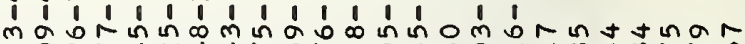

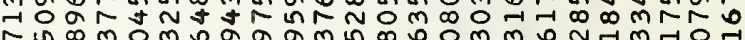

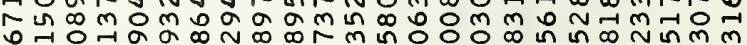

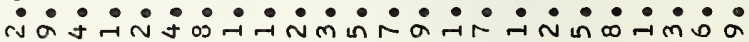

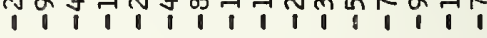

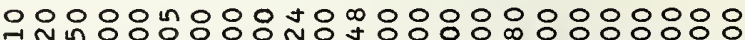

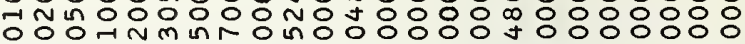

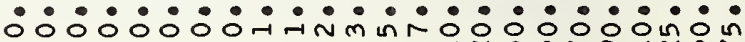
Hnm

000000000000000000000000 $\dot{N} \dot{N} \dot{N} \dot{N} \dot{N} \dot{N} \dot{N} \dot{N} \dot{N} \dot{N} \dot{N} \dot{N} \dot{N} \dot{N} \dot{N} \dot{N} \dot{N}$

N N N N N N N N N N N N N N N N N N N N N

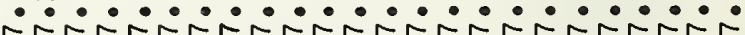

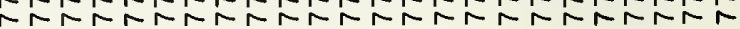

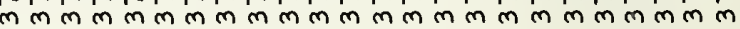


거다

닝

000000000000000000000000

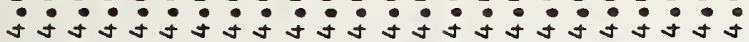

ม ต

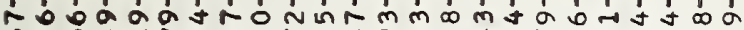

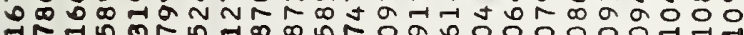

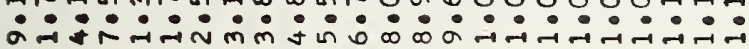

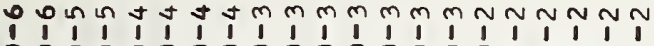

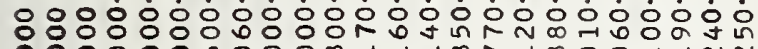

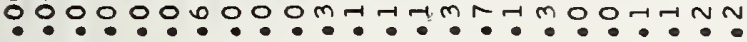

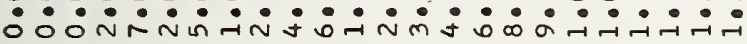

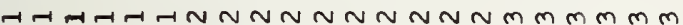

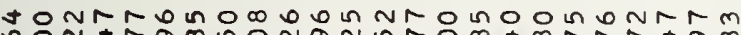

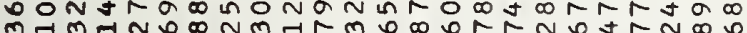
m

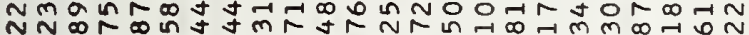

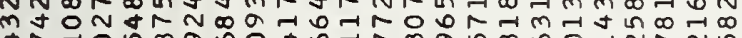
tr

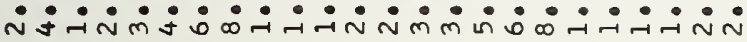

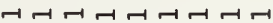

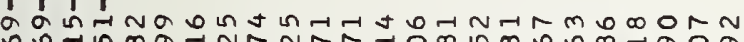

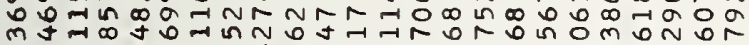

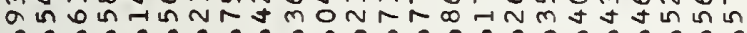

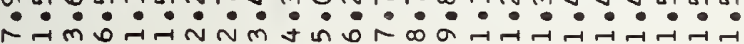

$\overrightarrow{1} \overrightarrow{1}$

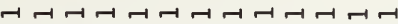

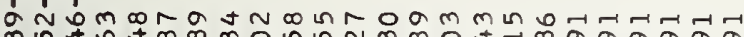

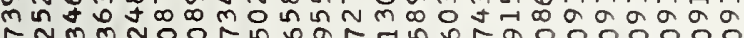

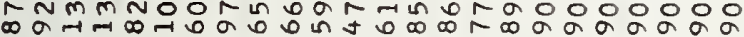

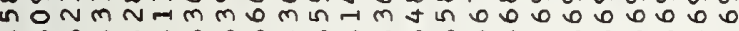

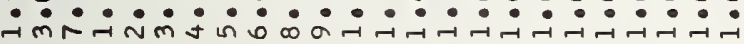

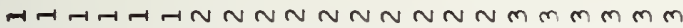
mar $N$ N NNat

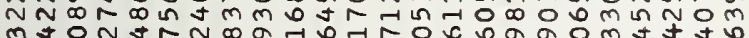

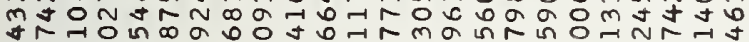

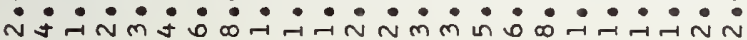

$2-1+1+1$ オ $\infty N+0 m N R \infty m$ t

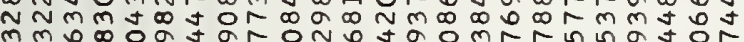

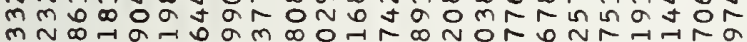

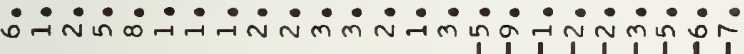

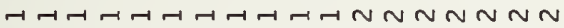

O D RO O-1 a d N N

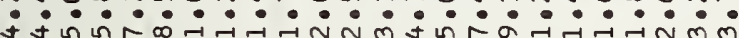

nป

$\neg-\neg-1$

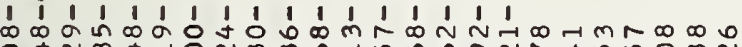

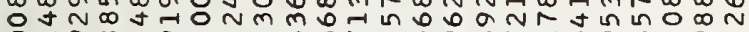

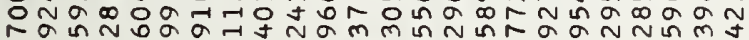

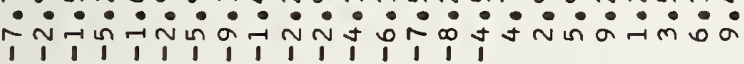

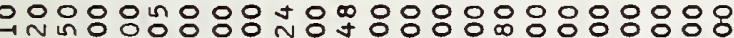

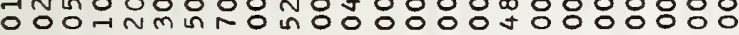

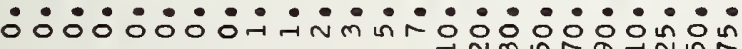
$\rightarrow$ N

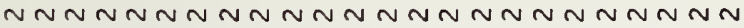

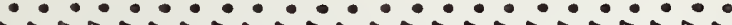

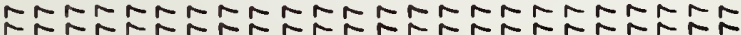

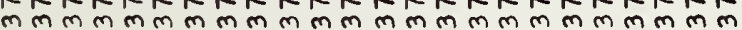

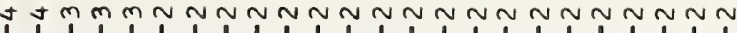

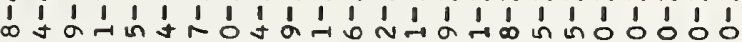
$\infty$ d

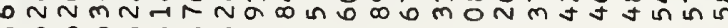

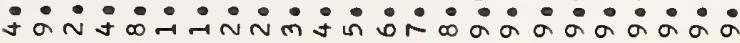

- 0 innा

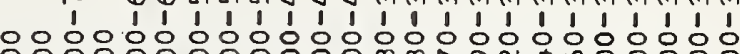

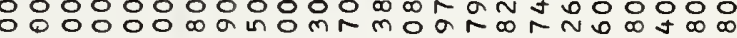

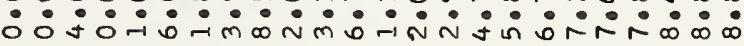

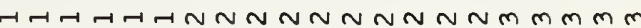

a moom 0 NOL

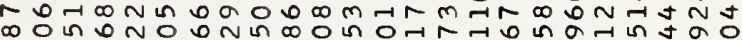

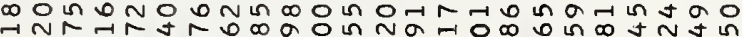
G y

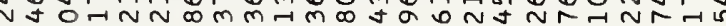

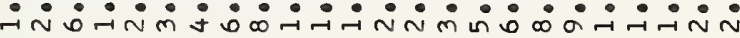

11111

- 0 ON 虽

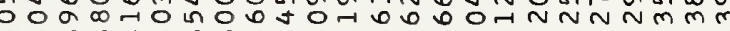

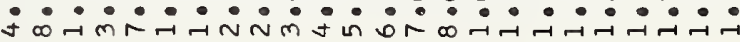

İ

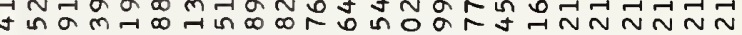

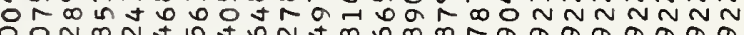

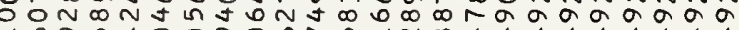
- ba

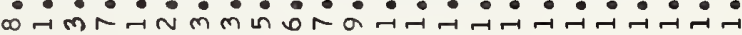

G INNNNNNNNNMmMmm

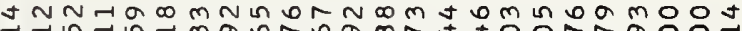

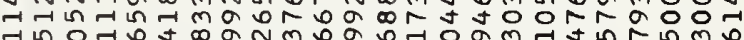

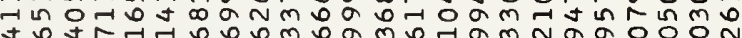

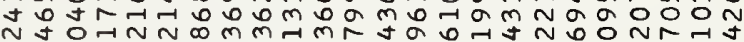

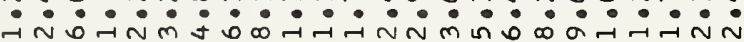

NNFFન D

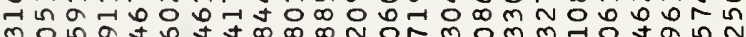

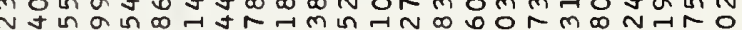

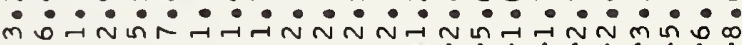
i 1 1 1 1 1 1 1

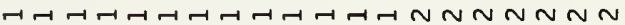

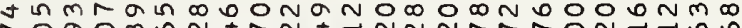

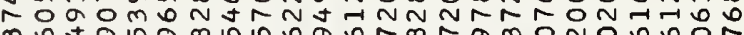

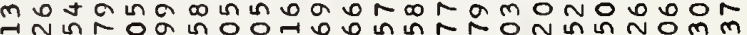
- $N$ in

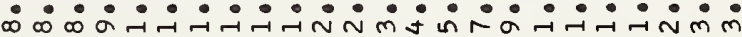
约 t

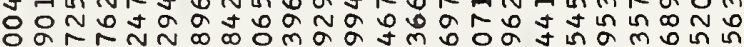

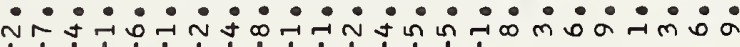
$\begin{array}{llllllllllllll}1 & 1 & 1 & 1 & 1 & 1 & 1 & 1 & 1 & 1 & 1 & 1 & 1 & 1\end{array}$

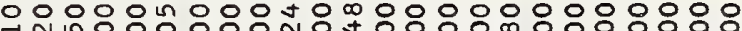

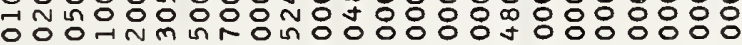

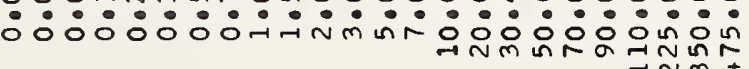

000000000000000000000000

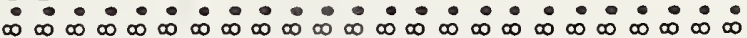

NNNNNNNNNNNNNNNNNNNNNNNN SiNa

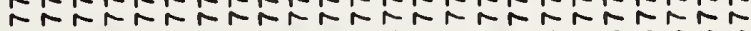

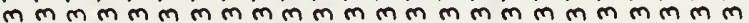




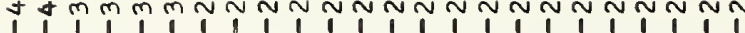

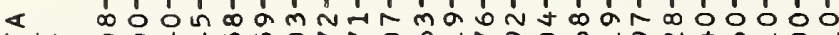

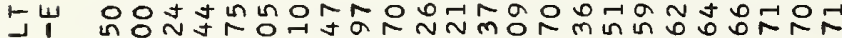
岁次 î́

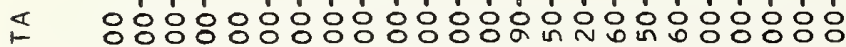

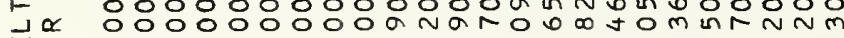

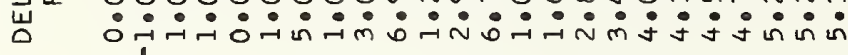

(1) In $00 a+m N m 00-10 m+00 m 0 m m 0$ $m a r+\infty$ in 0 in 0 in t

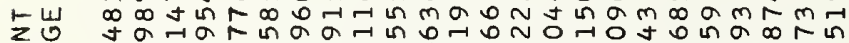
zu

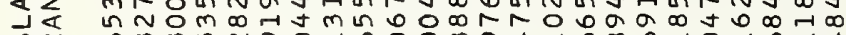
जळ

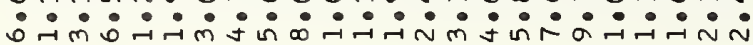
1)

뚱

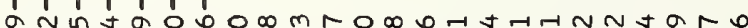
잉 $\frac{\alpha}{w}<$

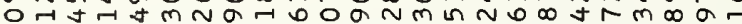
m m N

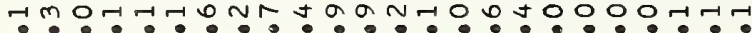

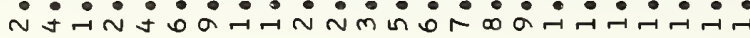

$i 1-1+1$

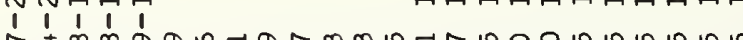

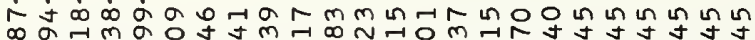

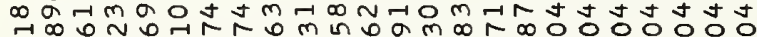
t m 0 - N N

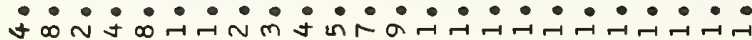

$i$

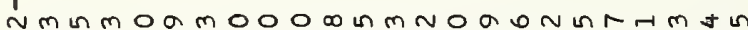
Nor NRanNatrm in t แก

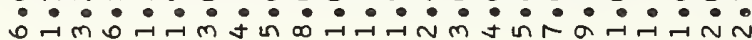

$\leftarrow \ll$

岀岌

$1 \pi N-1-1-1$

ir

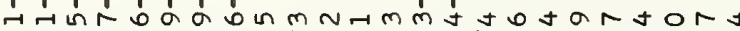
No에

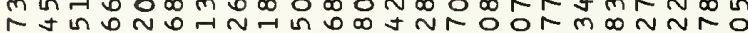

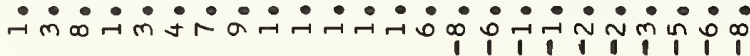

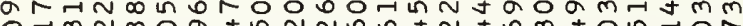

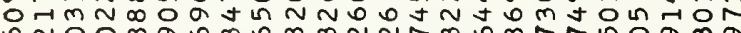

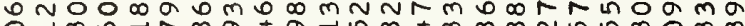

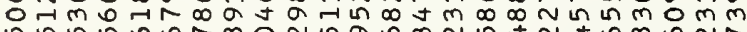

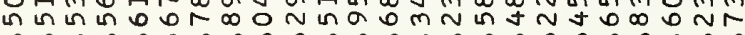

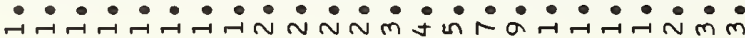

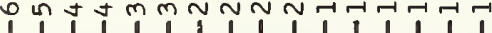

ศન

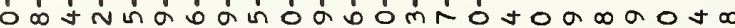

$\ll$

1

岁

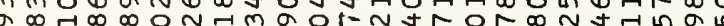

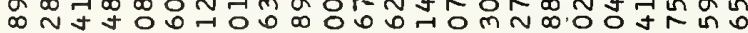

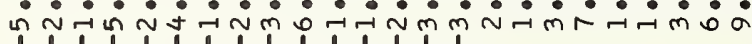

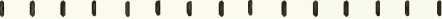

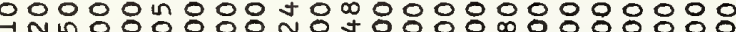
o o o n n m n

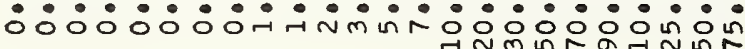

㞬

$\sum \frac{1}{2}$

000000000000000000000000 แ่

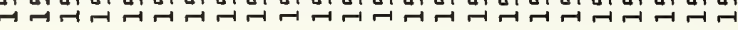

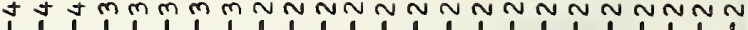

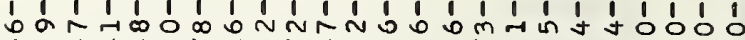

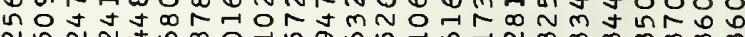
- : ? ? ? ? ? ? ?

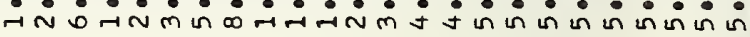

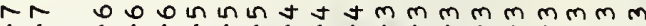

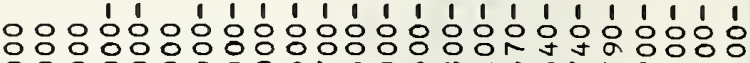

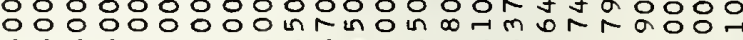

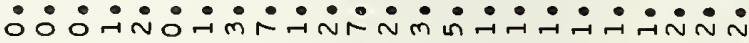
ना नानमननमN

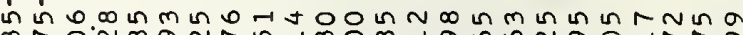

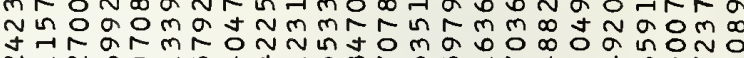
光年 mbo

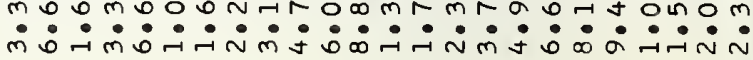

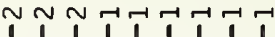

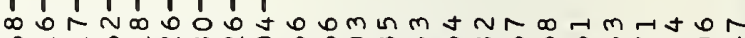
许

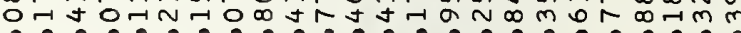

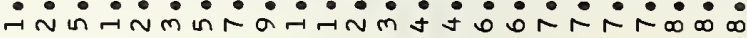

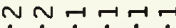

in

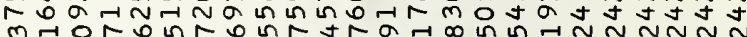

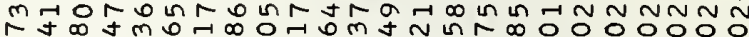

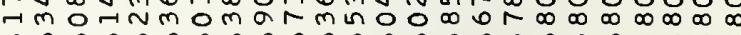
$\dot{\sim} \dot{j} \dot{\sim} \dot{j} \dot{0} \dot{-} \dot{-} \dot{\sim} \dot{m} \dot{j} \dot{\sim} \dot{r} \dot{\infty} \dot{\infty} \dot{\infty} \dot{\infty} \dot{\infty} \dot{\infty} \dot{\infty} \dot{\infty} \dot{\infty}$

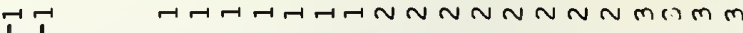

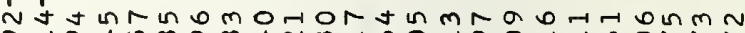

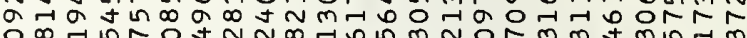

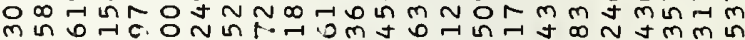

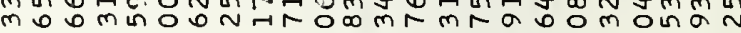

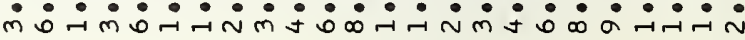

mNNN

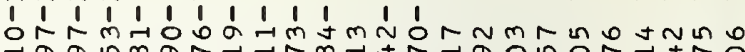

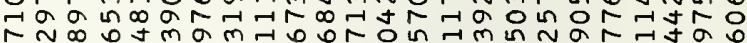

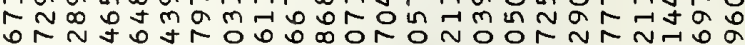

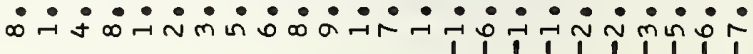

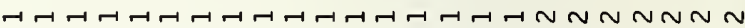

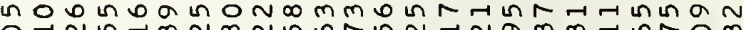

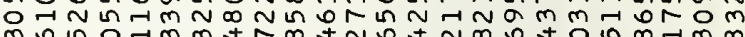

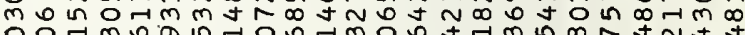

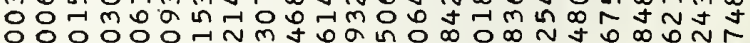

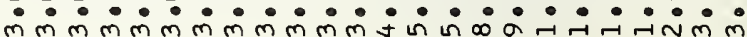

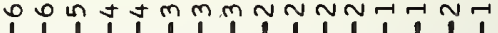

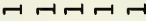

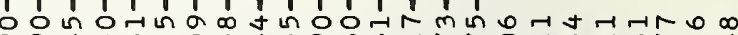

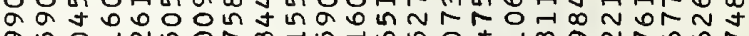
a n

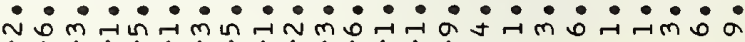
$\begin{array}{lllllllllllllll}1 & 1 & 1 & 1 & 1 & 1 & 1 & 1 & 1 & 1 & 1 & 1 & 1 & 1 & 1\end{array}$

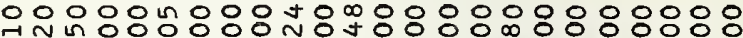

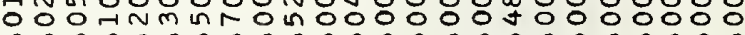

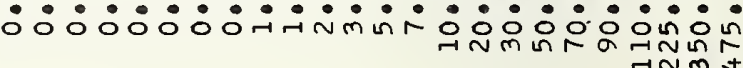

000000000000000000000000

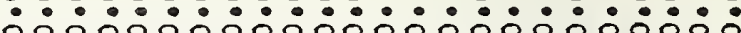

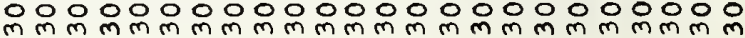

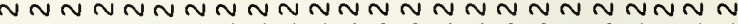
-

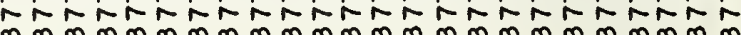

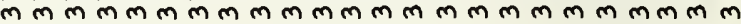


जJ

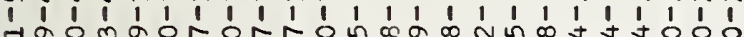
w

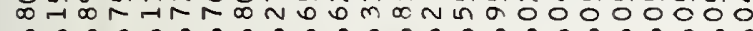

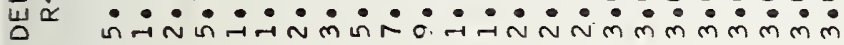

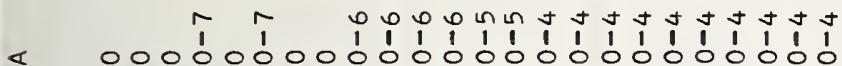
능ㅇㅇㅇㅇㅇㅇㅇㅇㅇㅇㅇㅇㅇㅇㅇㅇㅇㅇㅇㅇㅇㅇㅇㅇㅇㅇㅇㅇㅇㅇㅇㅇㅇㅇㅇㅇㅇ 10 0

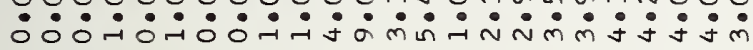

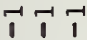
$-\pi-\neg-7-N \sim N N N$ N o o o n n

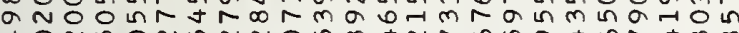

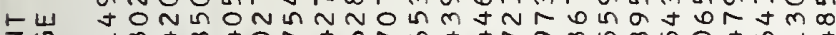
zu Iद mरam $\vec{\omega} \propto$

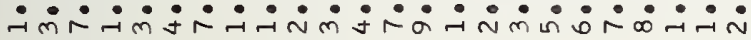

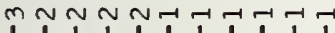

N 1100 닌 药

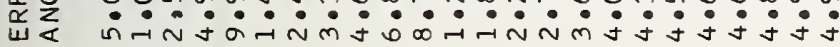
$n \sim \sim \sim-1-1-1$

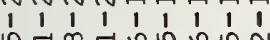

N

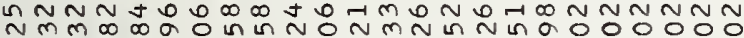

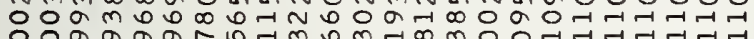
o 0 a a a

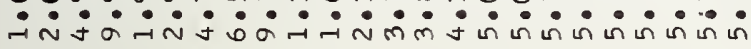

$17-1$

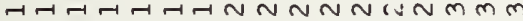

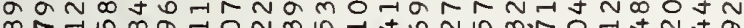

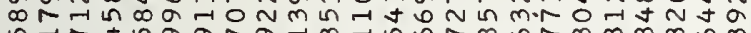
in $r$ t m

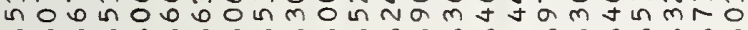

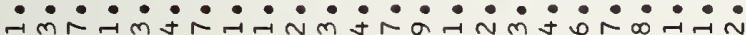

$\ll \ll$

$m m N N N-1-1-1-1-1-1-1$

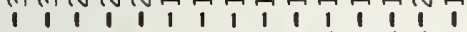

业岌

㟔岌

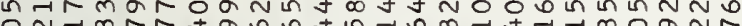
Nㅜㅇㅛ

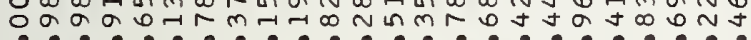

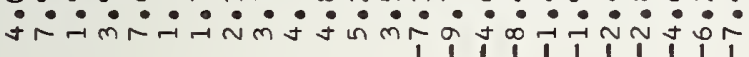
-

0 in $t N \infty$ in $N$ N $N$ O In

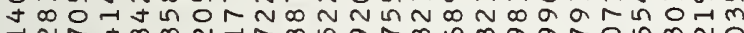

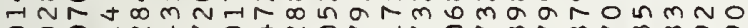
OOO

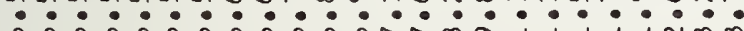

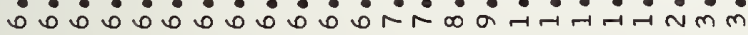

oromatammmmnNNN-1

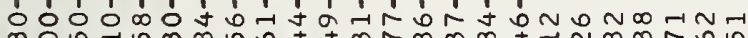
$\infty 0$ in m $m$ m - $N 0 \infty$ m 0 m n

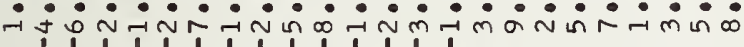

00000 in o o t om 00000000000

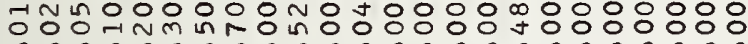

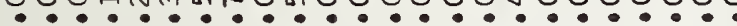

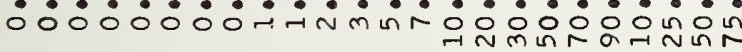

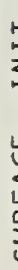

¿㟔

000000000000000000000000 in $\dot{0}$ in in in in in in in in in in in in in in in in in in in

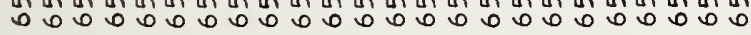

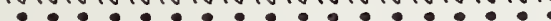

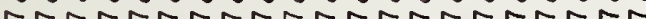

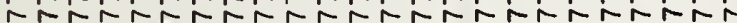

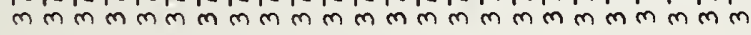

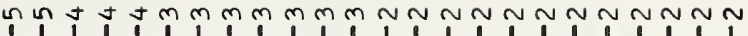

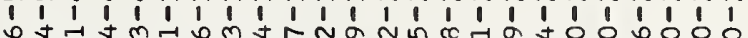

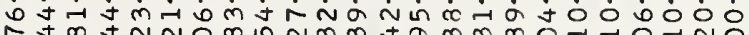

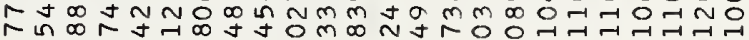

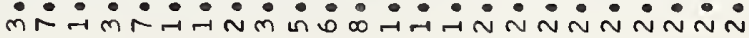

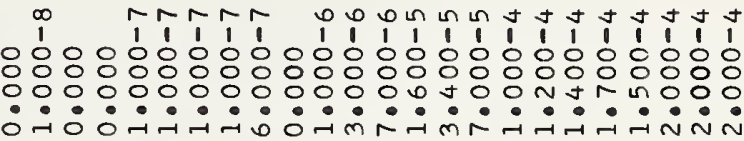

$\overrightarrow{1}-\overrightarrow{1}$

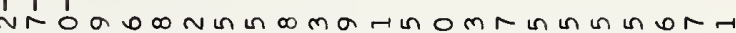

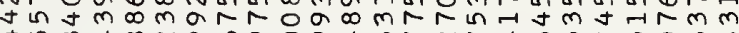

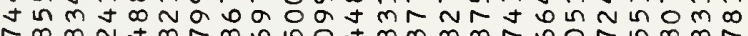

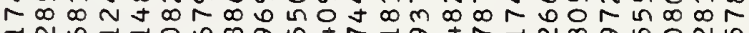

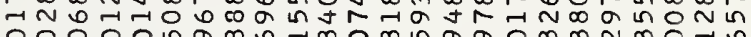
ㅇㅇㅇㅇㅇㅇㅇㅇㅇㅢ

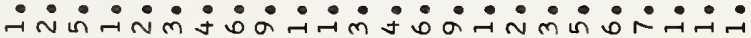

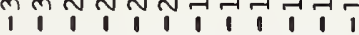

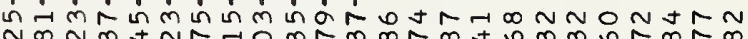

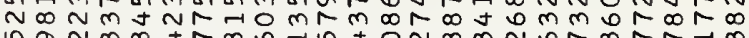

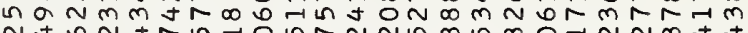

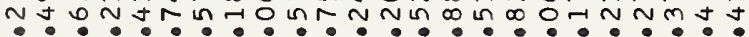
$\dot{m} \dot{0} \dot{m} \dot{0} \dot{\sigma} \dot{i} \dot{i} \dot{j} \dot{i} \dot{\infty} \dot{-1} \dot{-} \dot{\sim} \dot{\sim} \dot{m} \dot{m} \dot{m} \dot{m} \dot{m} \dot{m}$

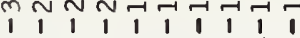

$\operatorname{rm} \infty \ln ^{-10} 0+-\infty \infty m-\infty \infty N m-1 N N N N$ ง $m$ ง

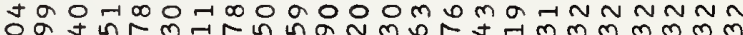

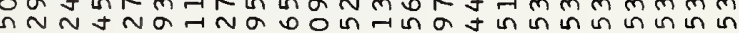

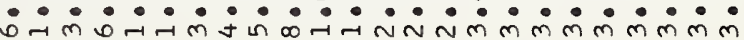

$\stackrel{1}{1} \overrightarrow{1}+1$

a t 0 a m onta $m \infty R N$ t ט人a

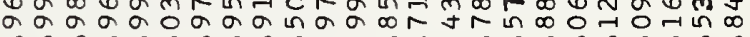

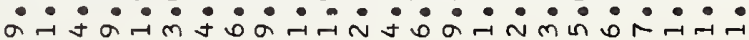

MN

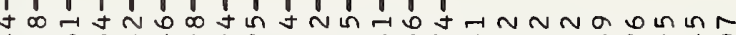

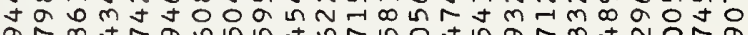
ar $\infty$ tra 0 in

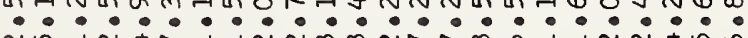

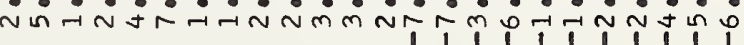
NNNNNNNNNNNNNNNNNNNNNNNN

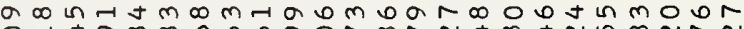

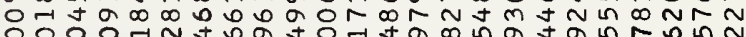

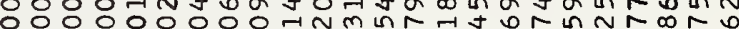

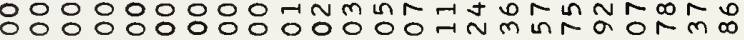

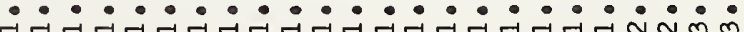

Don $n$ n

1 웅유 m

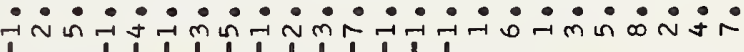

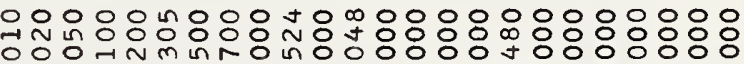

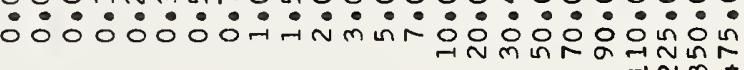
TnM

000000000000000000000000

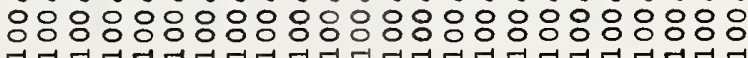

$N N N N N N N N N N N N N N N N N N N N N N$

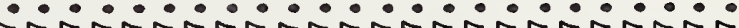
Nm $m \mathrm{~mm} m \mathrm{~mm} m \mathrm{~mm} m \mathrm{~mm} m \mathrm{~m} m \mathrm{~m} m \mathrm{~m}$ 
1
1

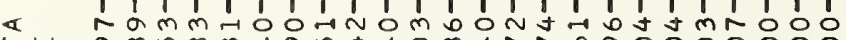

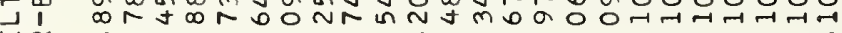

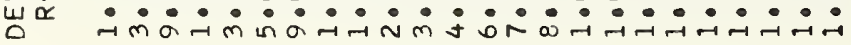

\section{$\infty \infty$ \\ Ar}

$\overrightarrow{5} \simeq$

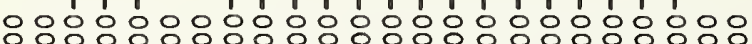

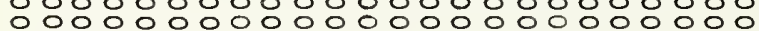
$\overrightarrow{0}$

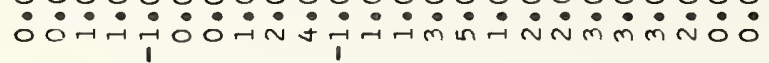
it

H- - $-1 \rightarrow \sim N \sim \sim N m$

b'j - $\infty$ ina 0 -1

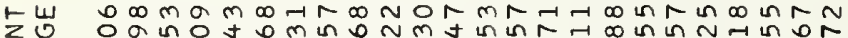

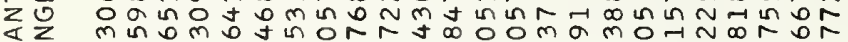
14

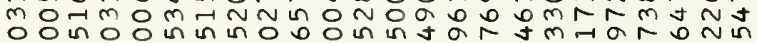

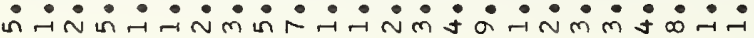

Mm N N N

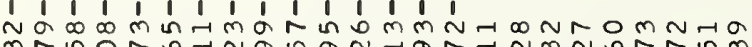

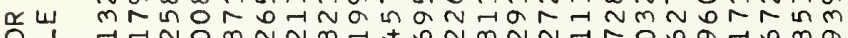

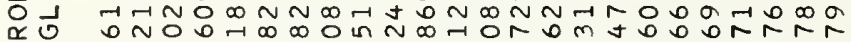

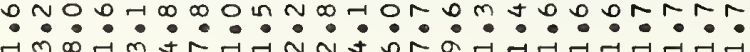

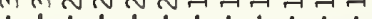

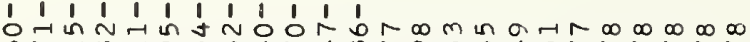

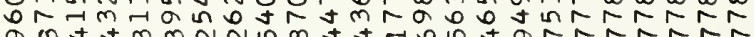

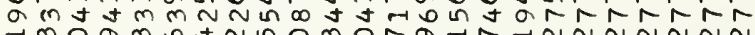

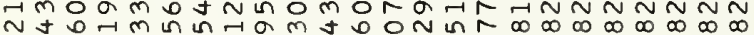

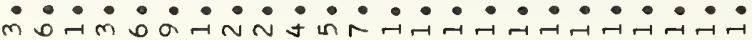

\begin{tabular}{l}
$u$ \\
2 \\
\multirow{5}{*}{}
\end{tabular}

NTMTH

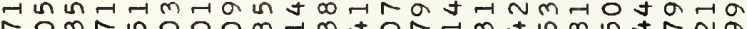

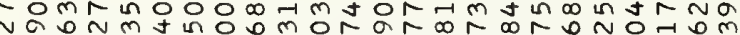
$m$ in om 000 组 $\alpha \infty$ t $a$ intata n

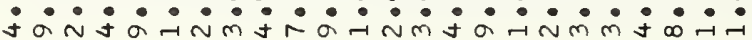

$m m m N N \approx N-1-1-1+1$

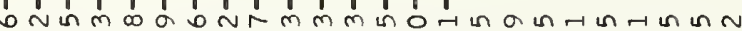

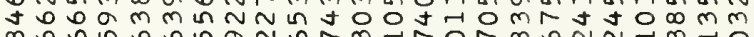

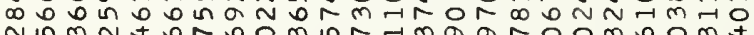

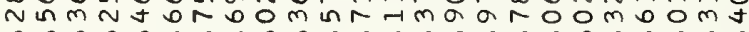

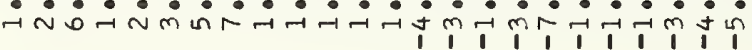

NNNNNNNNNNNNNNNNNNNNNNNN

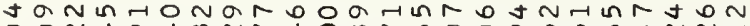

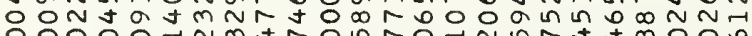

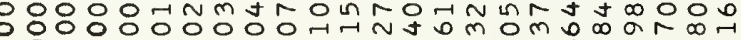

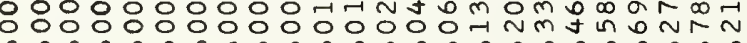

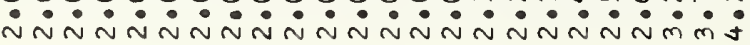

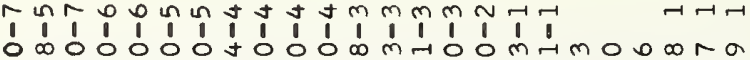

$\neg \sqcap \sqcap$

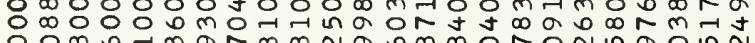
业I

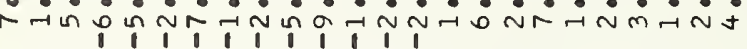

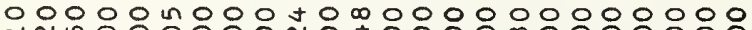

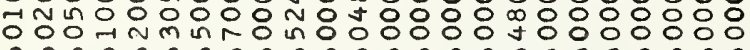

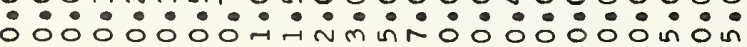
$00000000 \pi-N m$ in

ᄂ

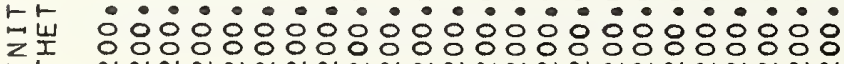

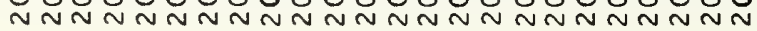

$\underset{\frac{u}{u}}{u}$

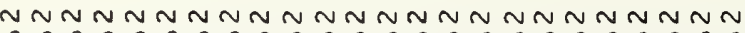

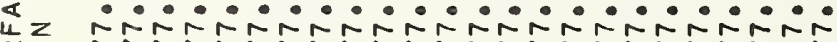
m

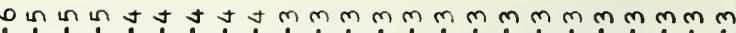

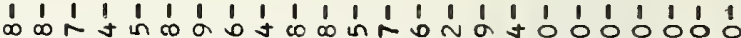
a m o $\alpha \infty$ o 0 b

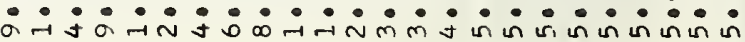

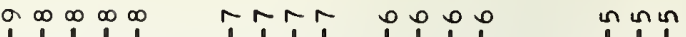

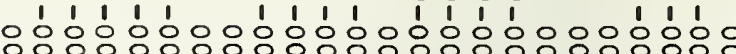

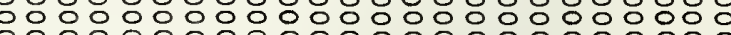
000000000000000000000000

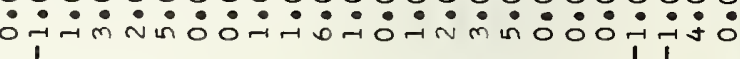
$\sim \sim \pi-1-1$ - $-1-N \sim \sim N N$ N J'

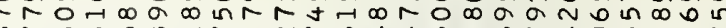
mrom in $m-1 N-1 N$ n

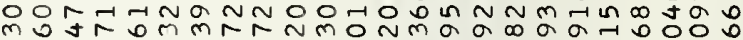

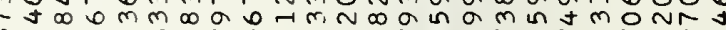
n

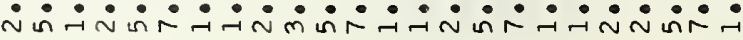

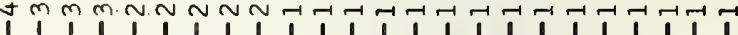

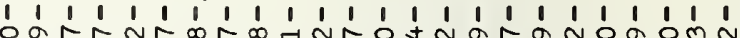

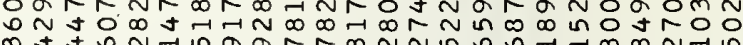

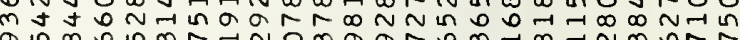

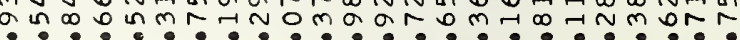

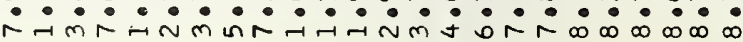

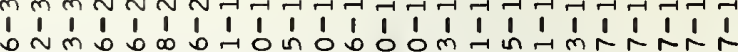

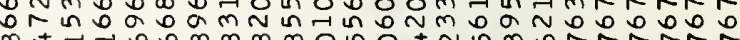

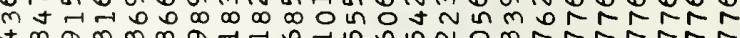

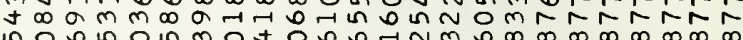

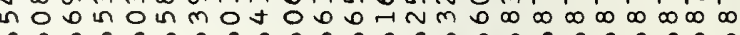

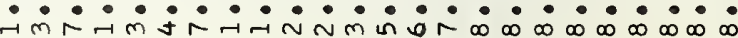

$\operatorname{in} 1 \mathfrak{1}$

-1 - $-14 \pi N$ N N N N N

o N

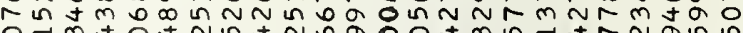

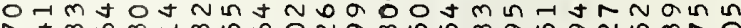
$r+\infty 0 m-\infty$ in 0 O $N a \infty$ in in $\infty a$ in $a n$ in $m \pi$

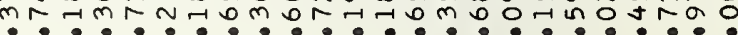

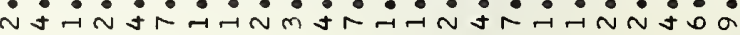

Amm $m \sim N N N N N N N N-1$

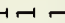

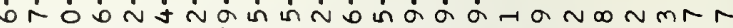

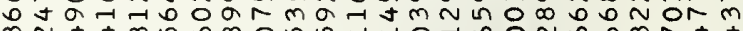

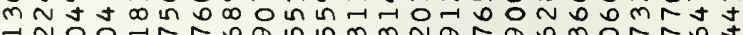
orr odnammon

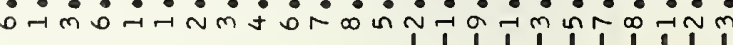
NNNNNNNNNNNNNNNNNNNNNNNN No 0 -1 $m$ -

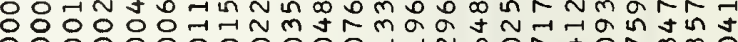

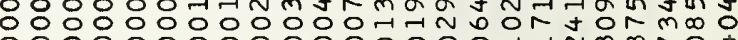
$0000000000000000001 \pi n m m$

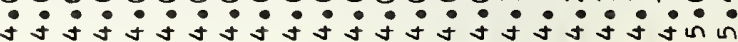

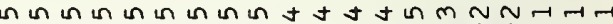

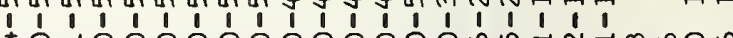
岤

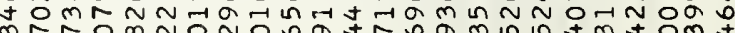
mrom nonomatroa m in un

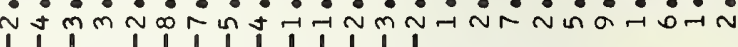

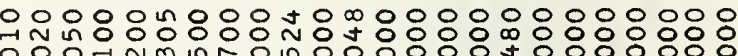
은?

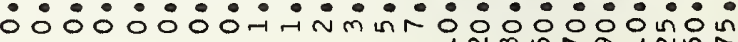
$\rightarrow N m$ J

000000000000000000000000

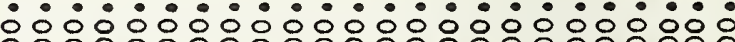

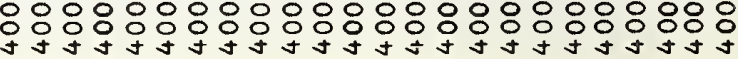

NNNNNNNNNNNNNNNNNNNNNNNN NR:

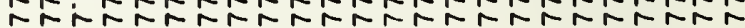
$\mathrm{mm} m \mathrm{~mm} m \mathrm{~mm} m \mathrm{~mm} m \mathrm{~mm} m \mathrm{~mm}$ 


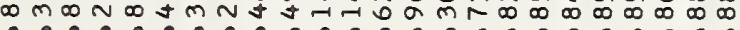

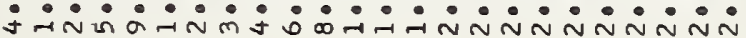

000000000000000000 $\stackrel{\leftarrow}{\leftarrow} \alpha$

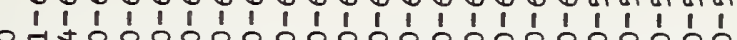

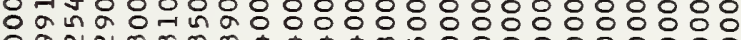
은?

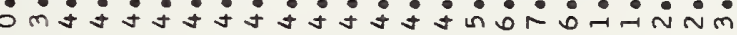

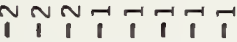

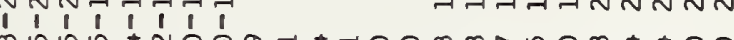

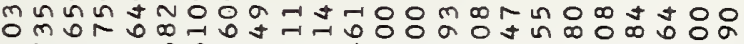

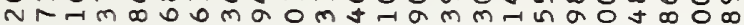

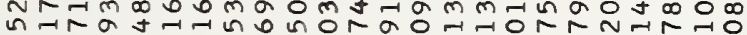

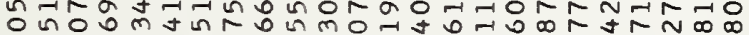

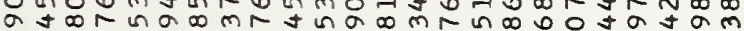

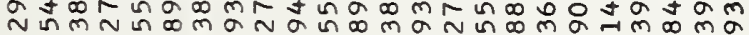

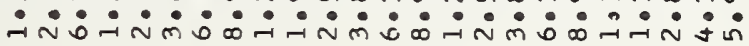

T.M

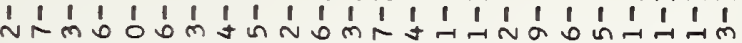

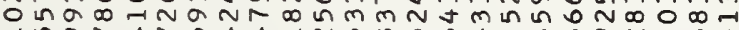

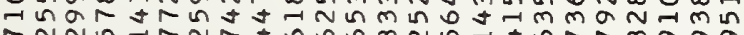
ก ก

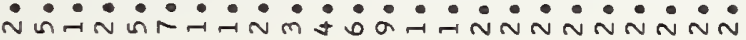

T

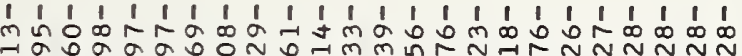

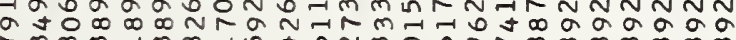

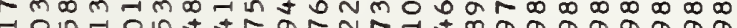
- O In

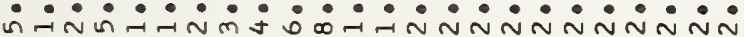

iิ

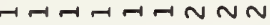

$u$
$\vdots$
5
5
0

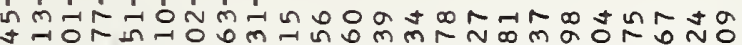

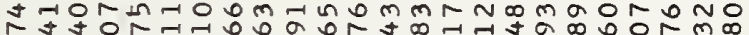

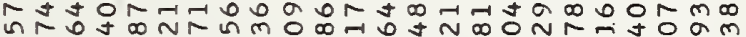
- na $\alpha$ แn

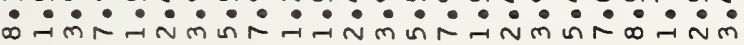

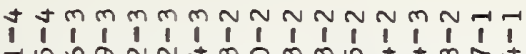

- noanntmomm心tanrtammtanr

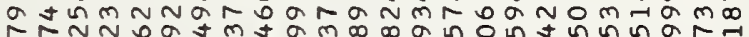

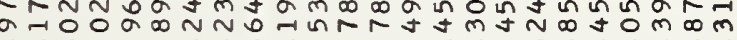

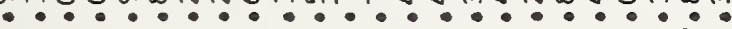
N N N N N N N N N N N N N N N N

OHM

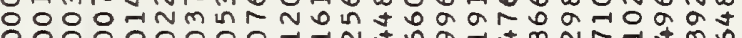
ㅇㅇㅇㅇㅇㅇㅇ은 잉ㅇㅇㅇㅇㅇㅇㅇㅇㅇㅇㅢ $000000000000000000017 n t$

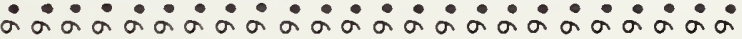

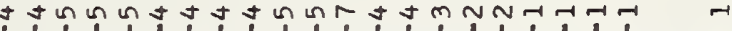

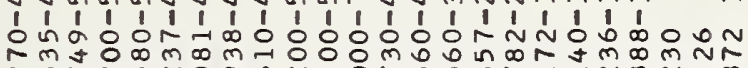

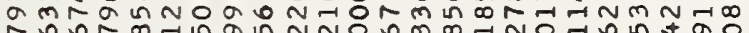

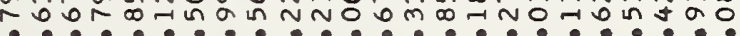

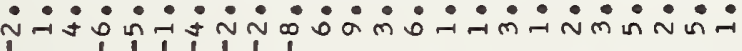

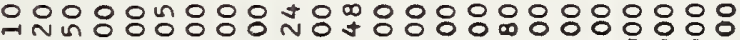
$000 \pi n m i n d$ in 00000070000000

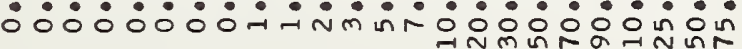

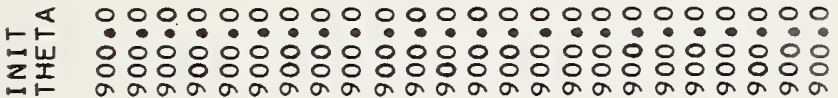

nNun

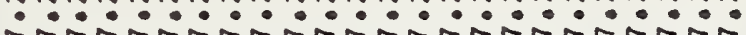

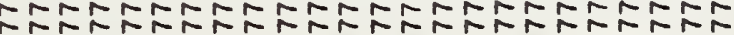

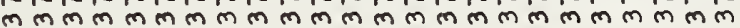



๒U

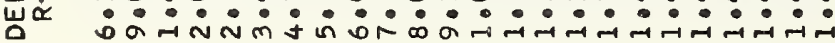

on m nntatammm

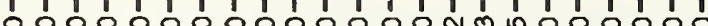

U.

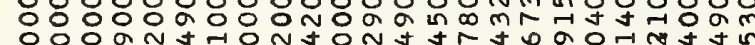

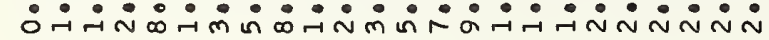

H-H H-HNNNNNNNNNNNNmmmmmm

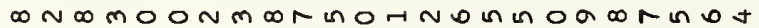
$N 0 m \alpha$ N N ᄂU N-U

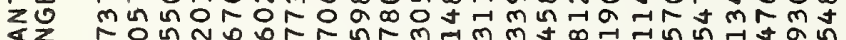
这

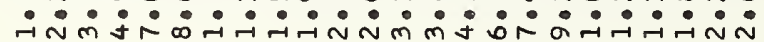

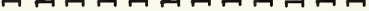

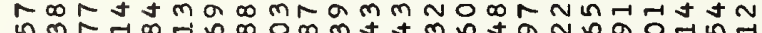
川 $m$ -

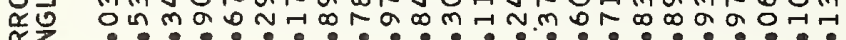

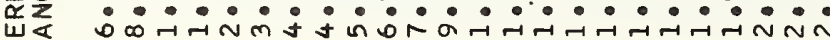

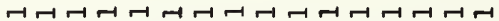

tNon $⿻$ n 거스네 o

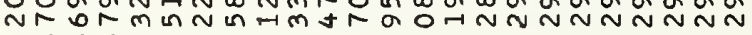

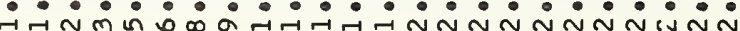

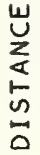

$\neg-\sqcap-\sqcap-N \sim N N \sim N N N \sim N N \sim m m m m m m$

NRamONaGRaN $m$ In NO In

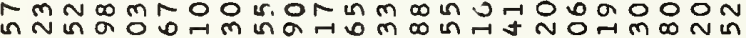
ก ำ?

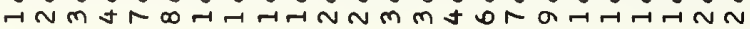

$i \overrightarrow{1}$

r-1

$\vdash$

岂㞬

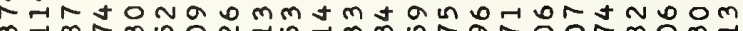

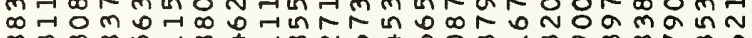

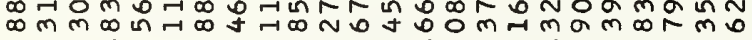

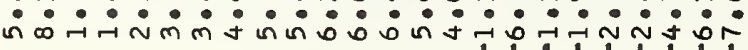

-

$\underset{⿱ ㇒}{\mathbb{I}}$

moO

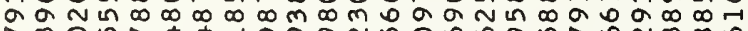


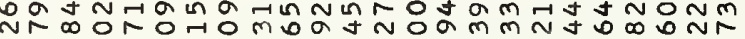

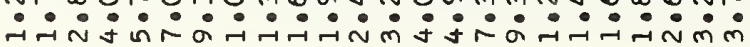

mmNNNF-1 I I

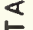
山I

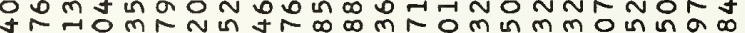
w

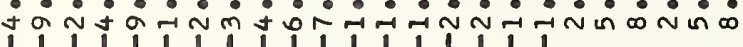
o 0000 m 000 t 0 mo 00000000000

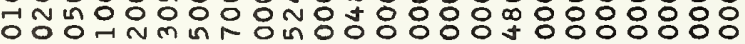
은?

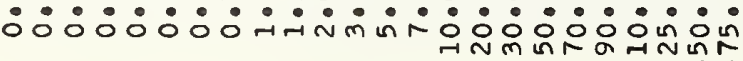
ல் $\dot{0} \dot{0} \dot{0} \dot{0} \dot{0} \dot{0} \dot{0} \dot{0} \dot{0} \dot{0} \dot{0} \dot{0} \dot{0} \dot{0} \dot{0}$

u<smiles>[Te]</smiles>
$\ll z$ $\stackrel{\alpha}{\supset}$

$\infty \infty \infty \infty \infty \infty \infty \infty \infty \infty \infty \infty \infty \infty \infty \infty \infty \infty \infty \infty \infty \infty \cdots$

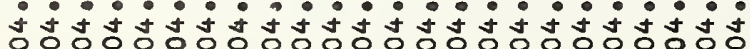

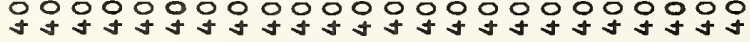

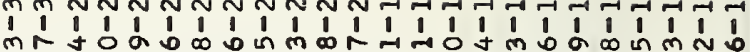

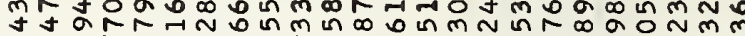

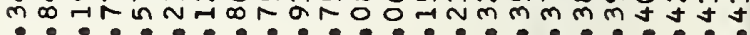

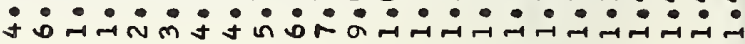

0 OnM J t t mmmmmmNNNNNNNNN

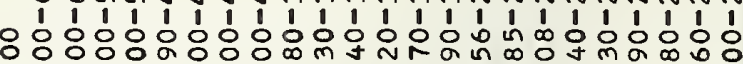

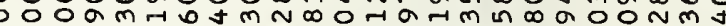

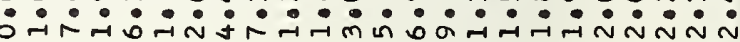
- $-1-\pi N N$ T

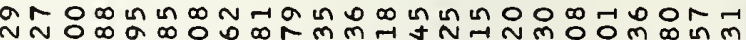
oN N

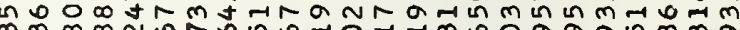
$m m \infty \infty N 0 r$

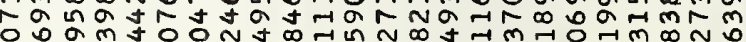

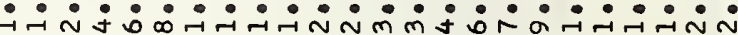

II

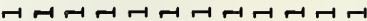

a

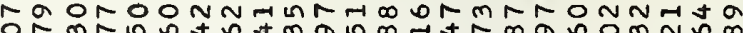

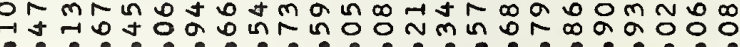

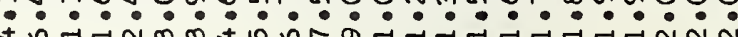

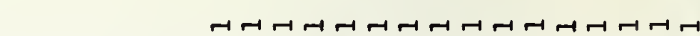
$\overline{1}$

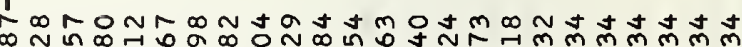

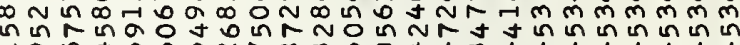

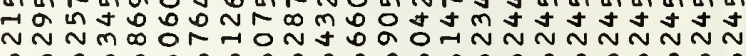

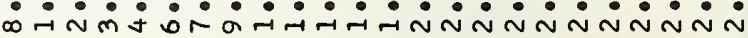

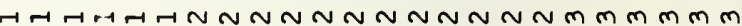

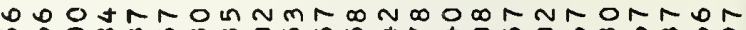
แn 0 에 $m m m$ in

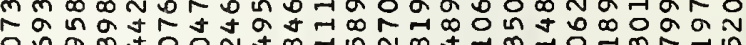

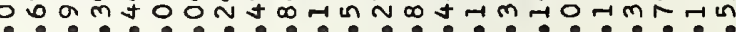

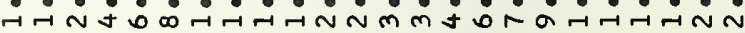

11

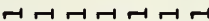

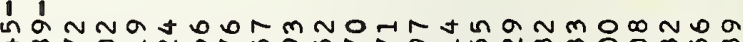
t $N$ O $N$ N

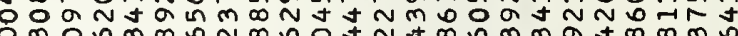
$m 00 m \infty \omega N \infty \omega 0 \pm N \pm \infty \omega m m a \pm \infty \infty m$

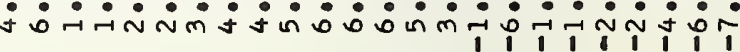

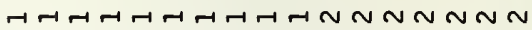

un $\infty$ o 0 - $a N m$ in

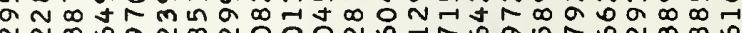

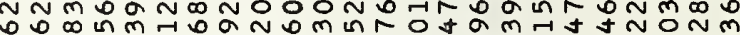

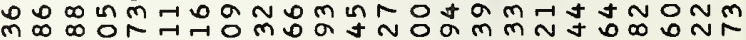

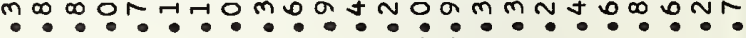

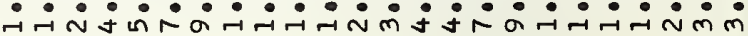

mm NNF-1-1-1

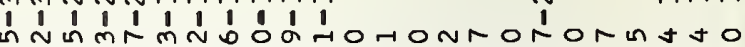

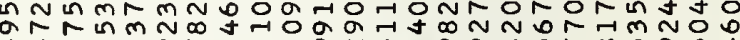

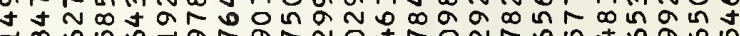

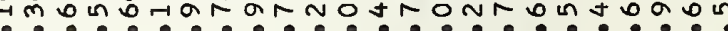
in $00000 \mathrm{~m} 000$ tomo00000000000 00000

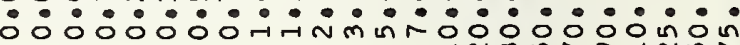
$\rightarrow$ in

แ

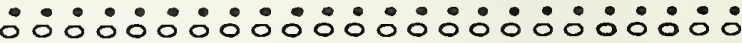

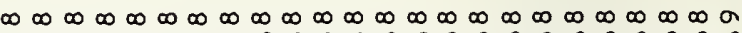

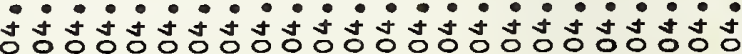

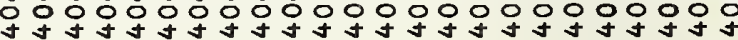




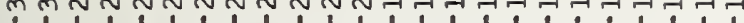

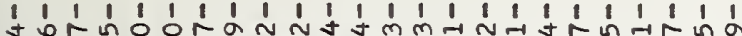
a

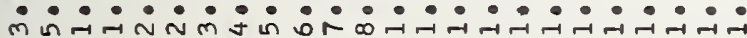

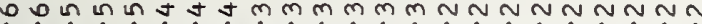

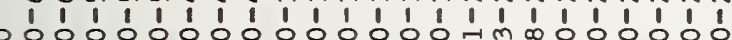

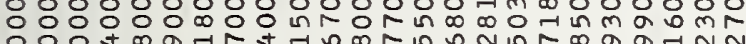

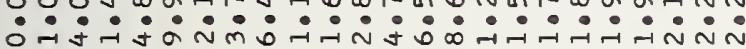

- $-1-1-1 N N N N N N N N N N N m m m m m m$

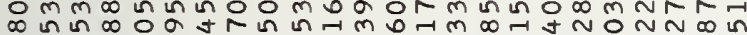

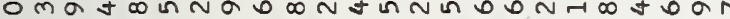

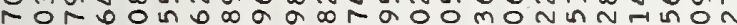
$\infty$ o

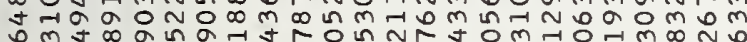

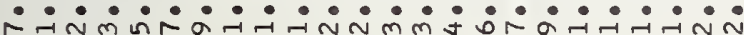

Tri त r Mm nnmRthnta

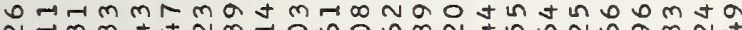

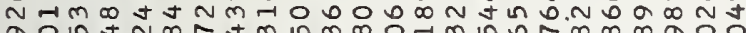

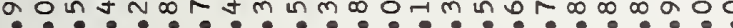

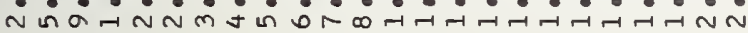
न

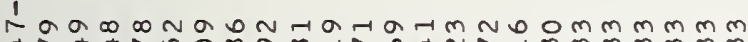
trytrin

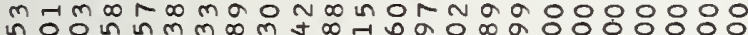

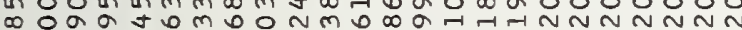

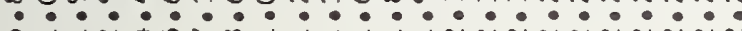
nก-

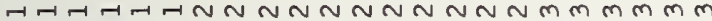

a m $m$-1 O O

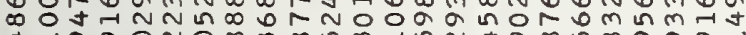

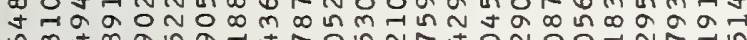

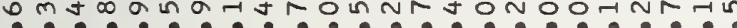

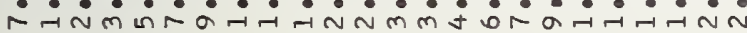

$7-1+1$

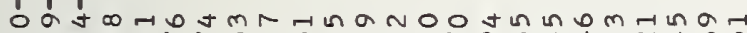

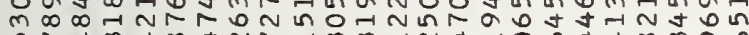
in

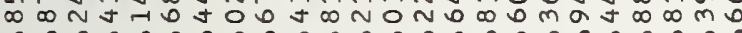

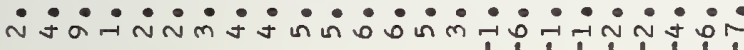

-

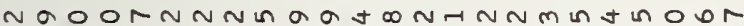
क R न 0 o tm $m$ T t

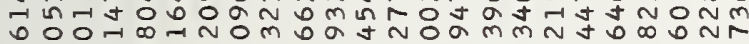

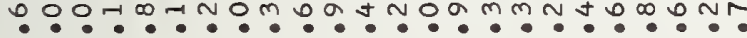

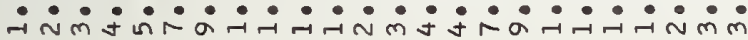

m $m \sim N \sim-1-1-1-1-1$

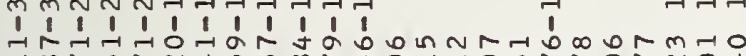
ăan 0 o

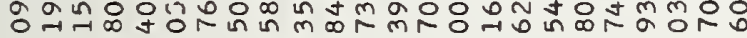
:

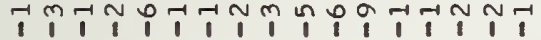

o o o o on 000 t on 000000000000 은 0 0 0 0 000 0 -

000000000000000000000000 मrन

$\infty \infty \infty \infty \infty \infty \infty \infty \infty \infty \infty \infty \infty \infty \infty \infty \infty \infty \infty \infty \infty \infty a$

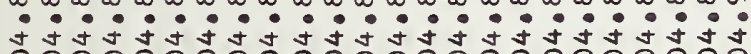

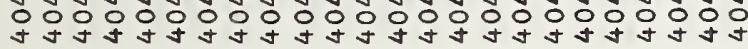

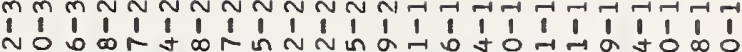

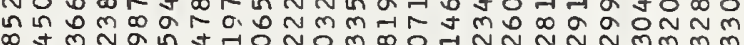

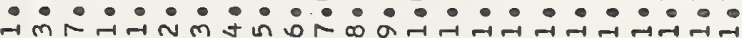

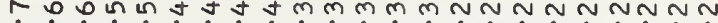

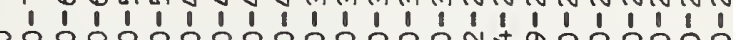

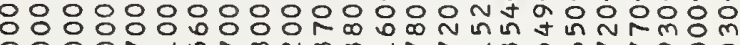
OOOON-T L

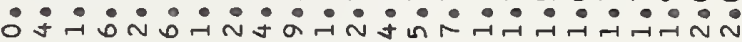

-1 $-1-\pi N N N N N N N N N N N m m m m m m$

추엉에

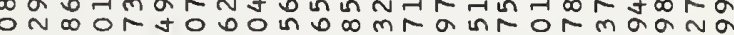

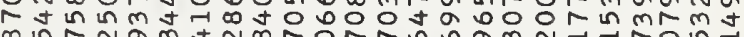
$\infty$ in Nam $N$ N

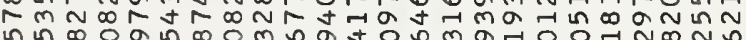

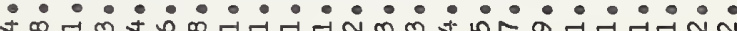

$\because \dddot{1}$

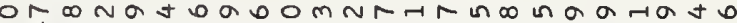
ntatantomamotomknoamka in

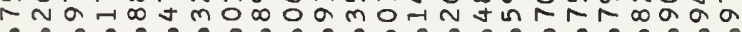

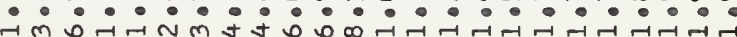

17

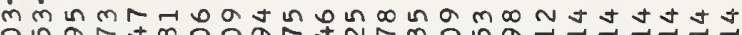
tom $\mathrm{m}$ in $\mathrm{m}$ moNay ○ 约 $m \pi \infty$ in $0 \rightarrow m$ in mbन

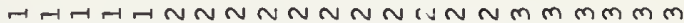

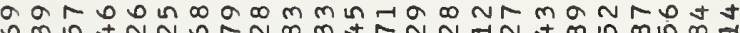

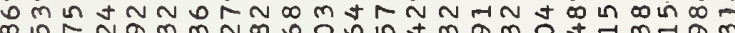

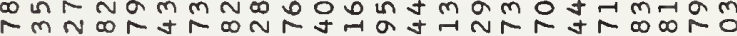

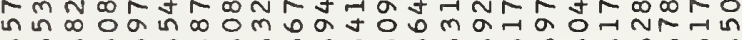

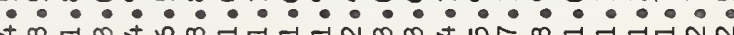

11

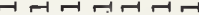

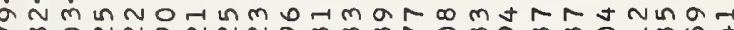

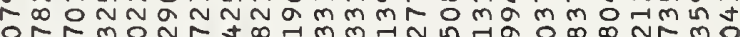
OR mon ก.

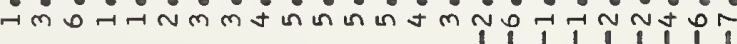

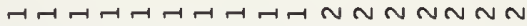

tanorom tatatank t

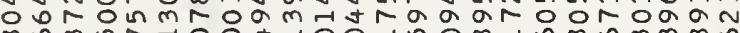

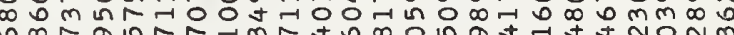
mojğ

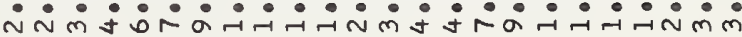

tmm $m$ I

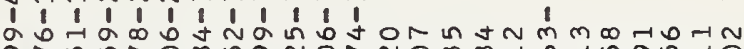

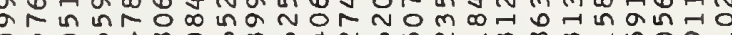
O amn $m$ n t o o b or

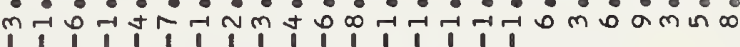
00000 in 000 t 0 mo 00000000000

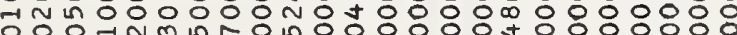
00 年

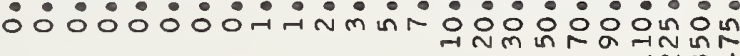

000000000000000000000000 NNN N

$\infty \infty \infty \infty \infty \infty \infty \infty \infty \infty \infty \infty \infty \infty \infty \infty \infty \infty \infty \infty \infty \infty a$

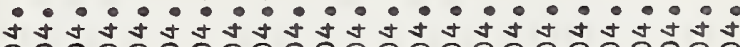

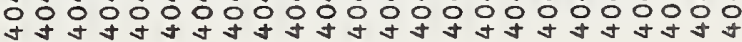




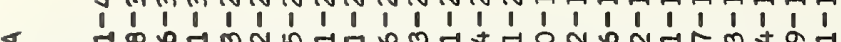

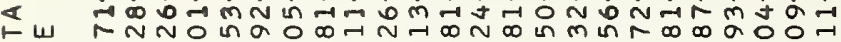
$11 \infty \alpha$ in $\tilde{w} \ltimes$

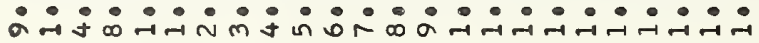

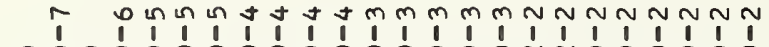

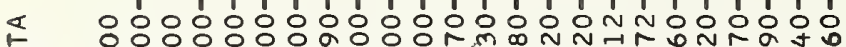

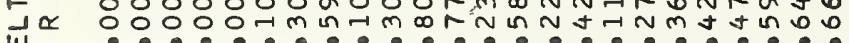

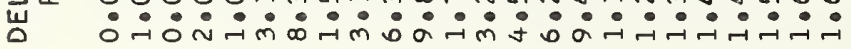

$\neg-\neg \sqcap-\neg N \sim N N \sim N N N N N m m m m m m$

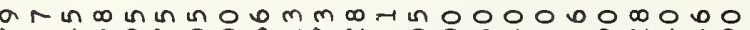

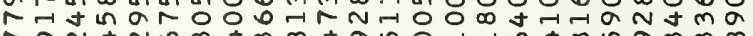
レU ZU

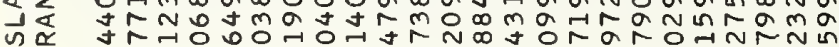

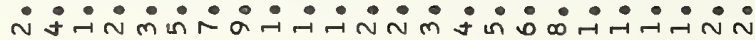

N 1 1

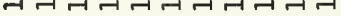

$\stackrel{\sim}{\circ}$

은

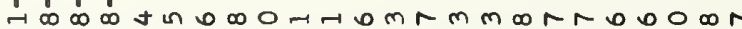

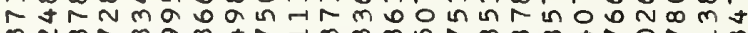

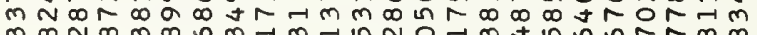

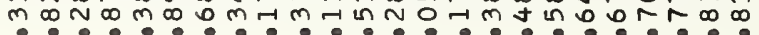

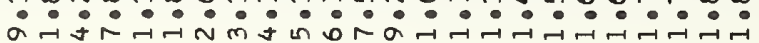

$1+1$

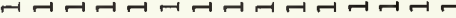

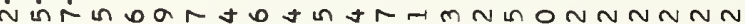

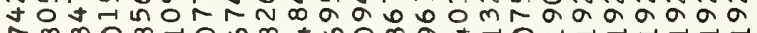

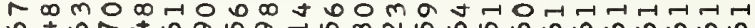

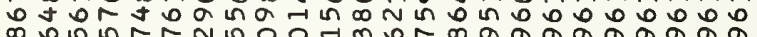

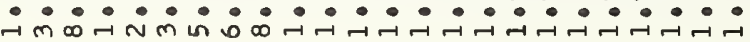

Hન-

$u$
$z$
$\vdots$
5
0

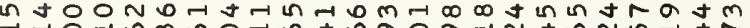
거더엉요 trnotmotatrmo

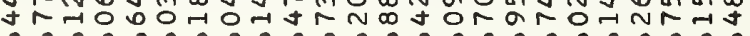

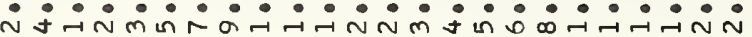

iิ

$<<$

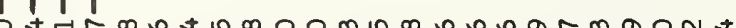

Ju

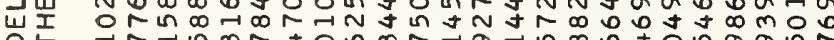
? ? ? ? ? ? ? ? ? ? ? ? ? ?

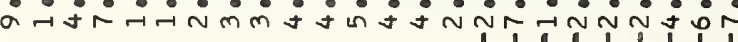

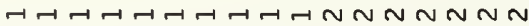

$\underset{⿱}{\mathbb{I}}$

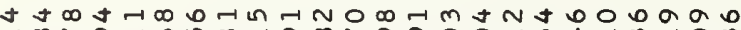

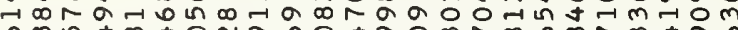

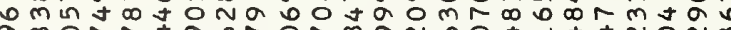

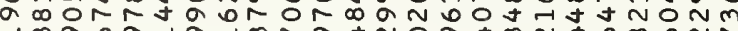

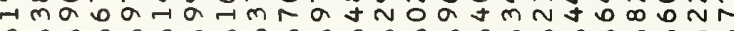

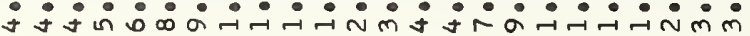

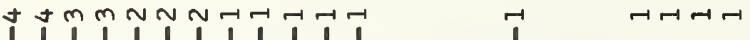
กn

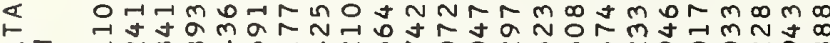
ᄀI F⿹勹弋

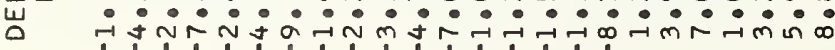
0

< 000000000000000000000000 不出

$\infty \infty \infty \infty \infty \infty \infty \infty \infty \infty \infty \infty \infty \infty \infty \infty \infty \infty \infty \infty \infty \propto$

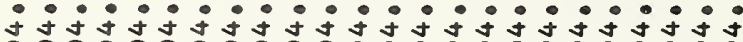

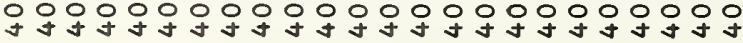

A.

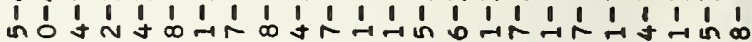

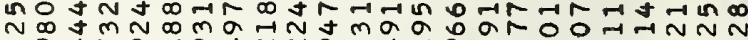
odaranatnnantha 0 mo 0000000

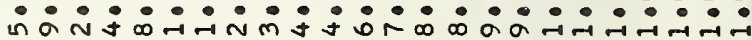

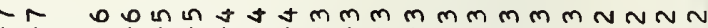

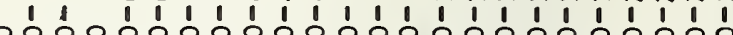

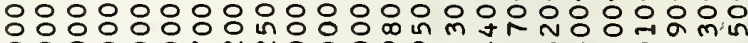

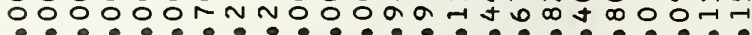

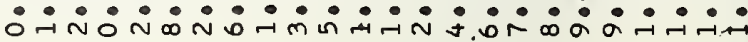

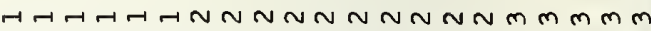

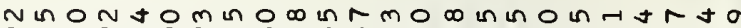

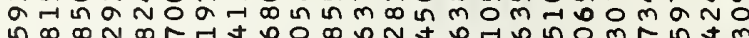

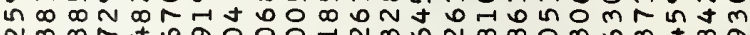

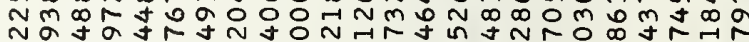
ง 0 o

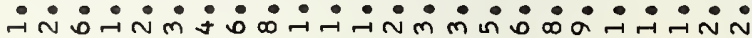

i $N \rightarrow-1$

A

ntmom $m$ t in $\alpha \sigma \alpha$ N

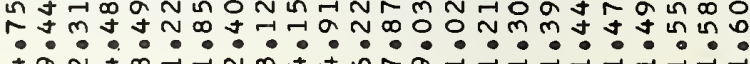

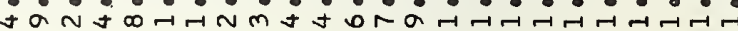

i

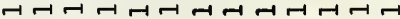

a구에

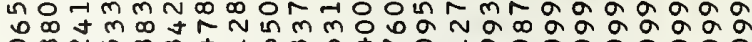

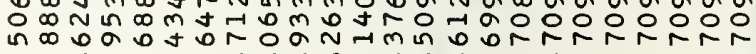

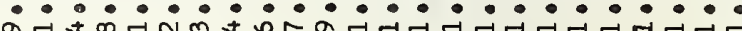

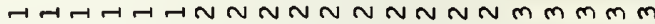

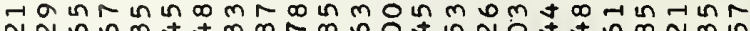
Nละ์

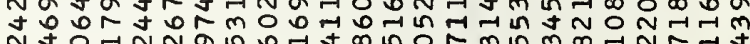

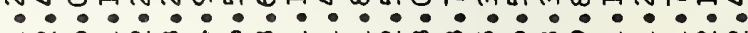

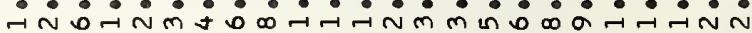

$2 \sim-1-1$

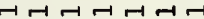

0 0 or nt

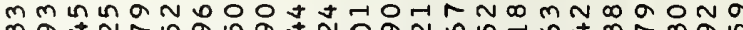
matnr una inat Noan in

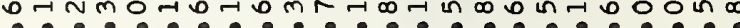

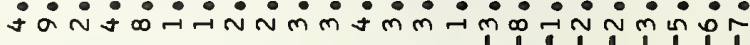

-

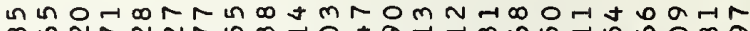

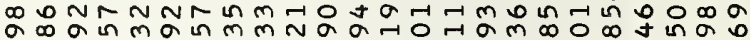

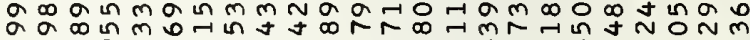
ôึ

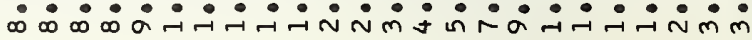

๓ก Im

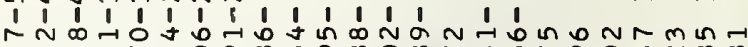

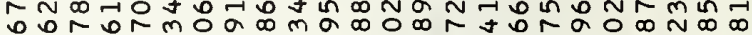

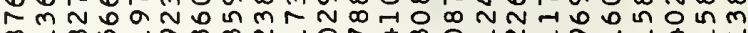

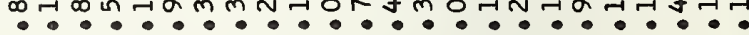

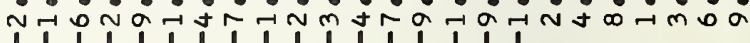

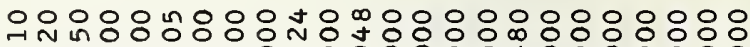

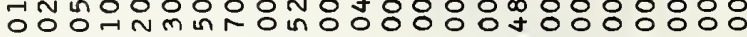

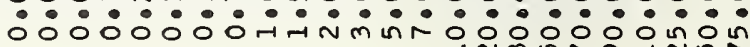

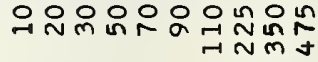

000000000000000000000000

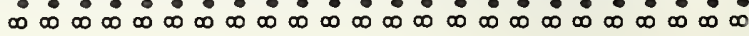

$\infty \infty \infty \infty \infty \infty \infty \infty \infty \infty \infty \infty \infty \infty \infty \infty \infty \infty \infty \infty \infty \infty a$

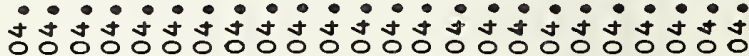

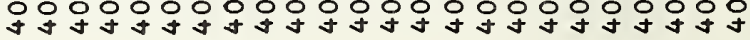




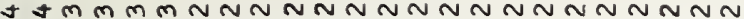

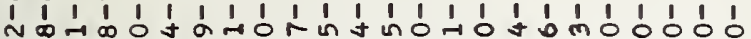

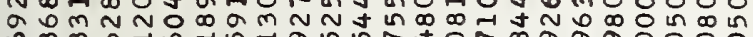

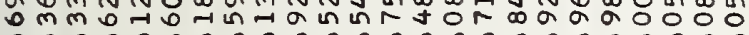

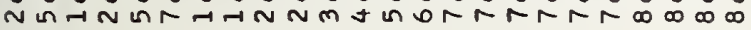

- N 00 innm

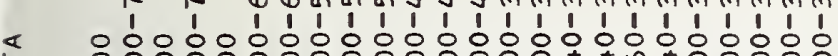
I w

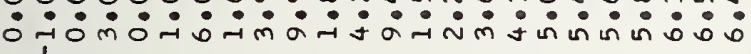
1 in $m N$ n $N-1$ in 0 in oñ

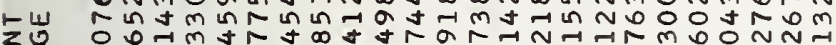

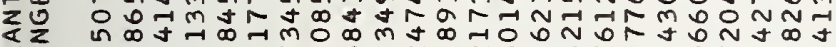
作 กิm

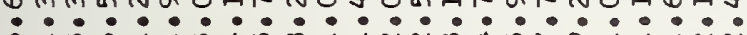

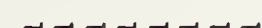

I

选

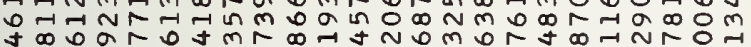

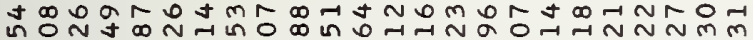
ำำง

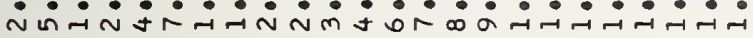

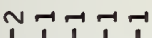

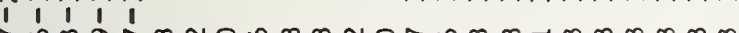
†

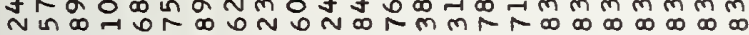

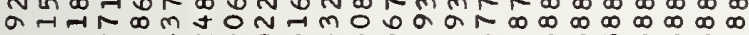

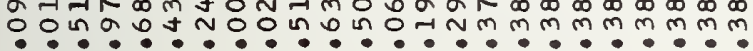

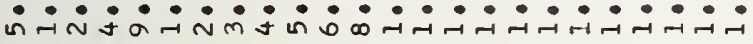

感

11

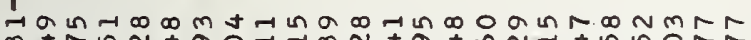
m t ț

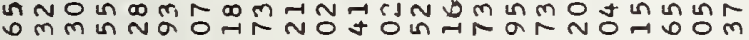

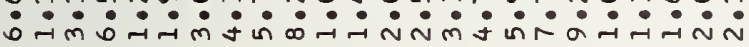

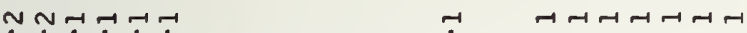

$\ll \ll$

$\vdash \leftarrow$

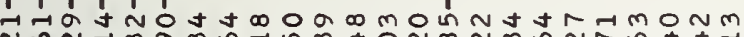
NoN

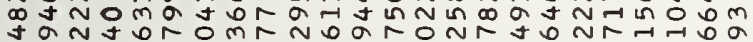

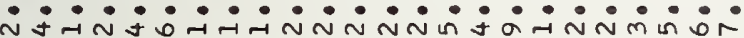
t $\infty$ in MDOONMNNG in o

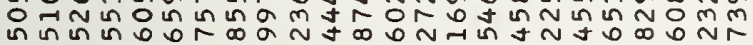

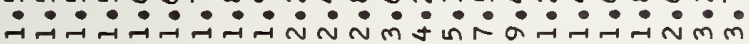

in

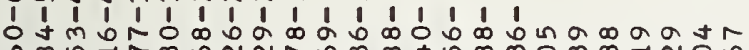
舟

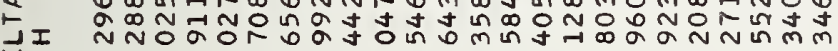

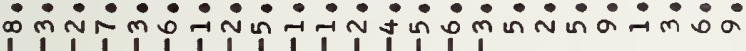

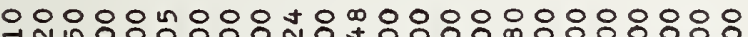

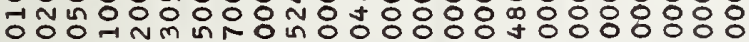

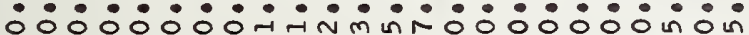
$00000000 \pi n m^{\circ}$

000000000000000000000000

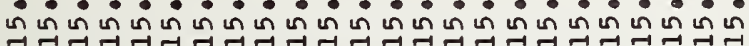

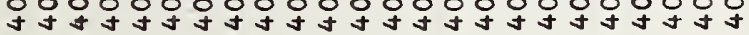

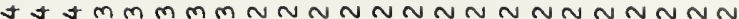

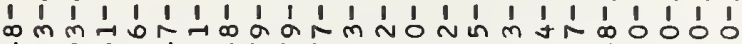
tám

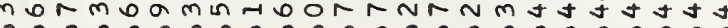

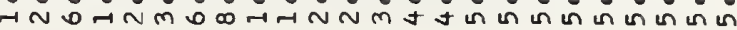

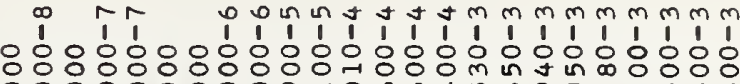

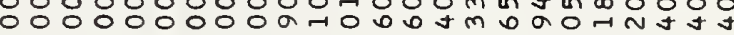

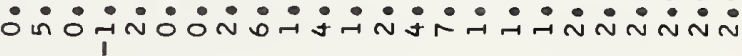

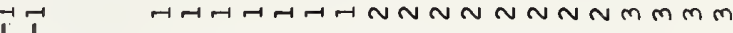
No 0 o in t N N

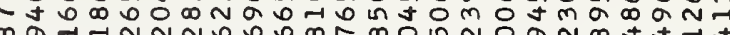
政 సN

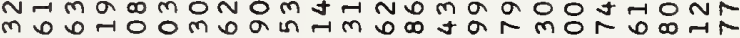

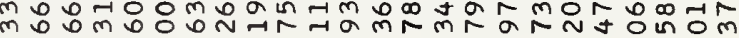

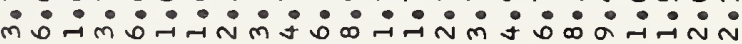

iv

$m b$ in $-1 m a b y 0$ t

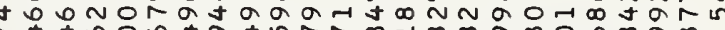
N

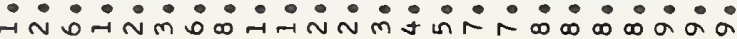

$\operatorname{1n} 1-1-1+1$

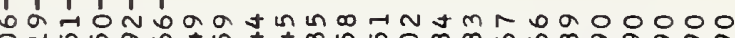

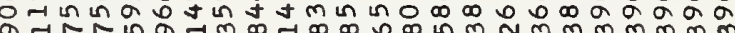

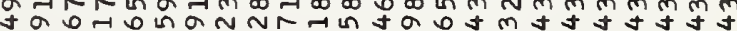
ง in on

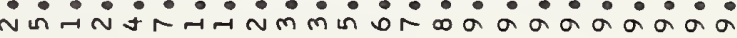

II

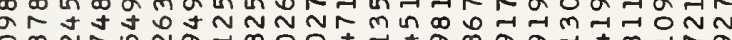

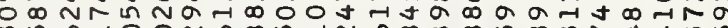
m

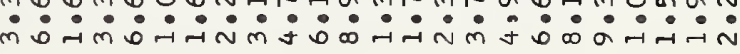

NN N-1 m

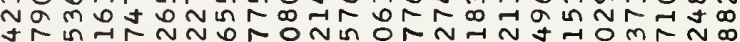

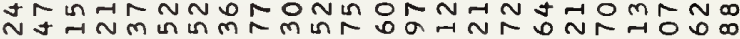

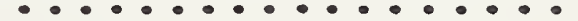

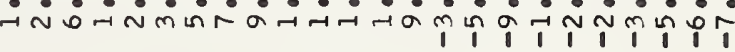

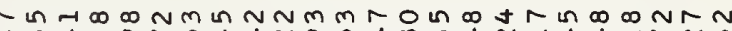

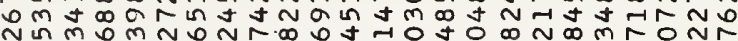

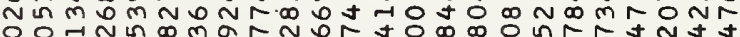

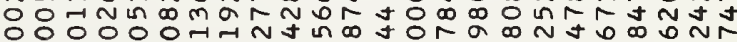

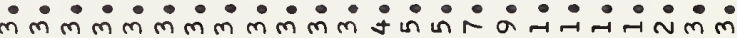

00 แ

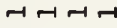

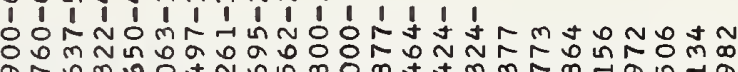

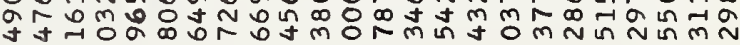

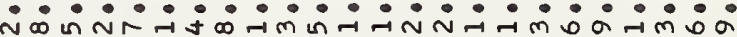

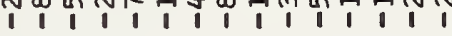

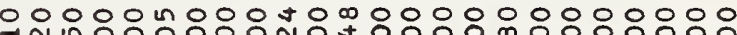

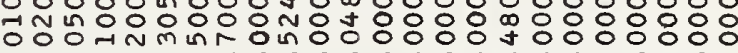

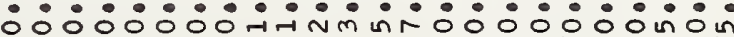
$\rightarrow$ m

000000000000000000000000

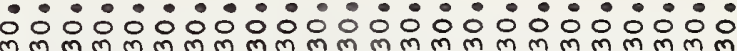

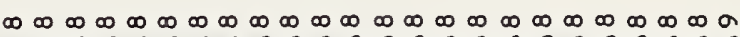

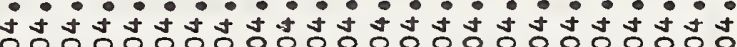

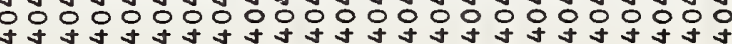




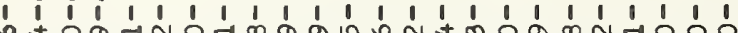

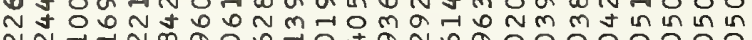
○

$\varangle$

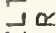
u

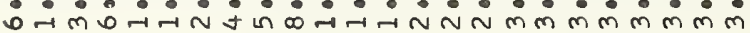

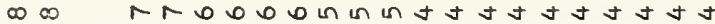

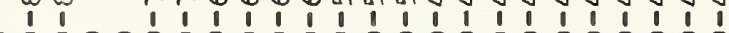

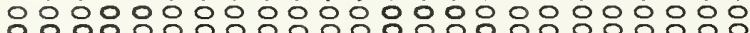

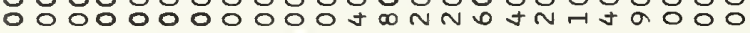
ல-

II

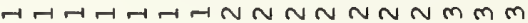

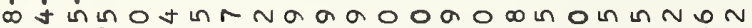

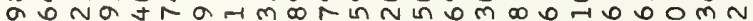

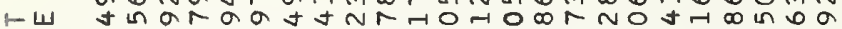

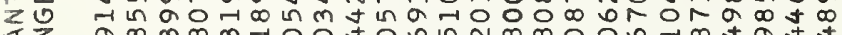

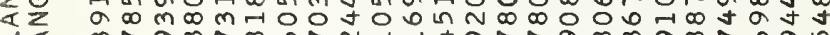

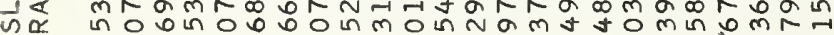

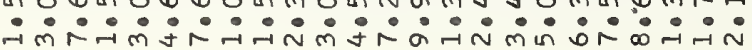

$m \sim N \sim-1+1-1$

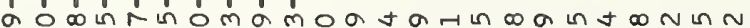

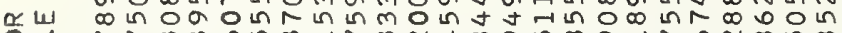

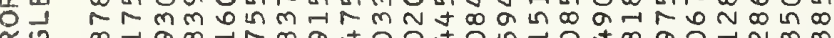
$\alpha$ \% $\omega<$

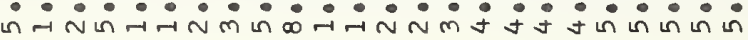

$N N$ N $N$-1

' in $\infty$ o min

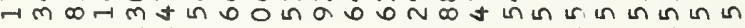
-

感

$\overrightarrow{1}-1$

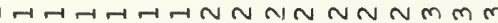

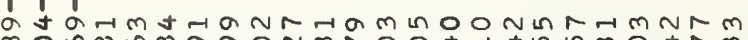

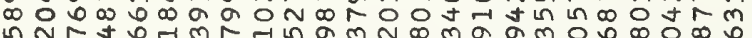

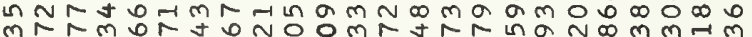

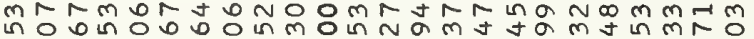

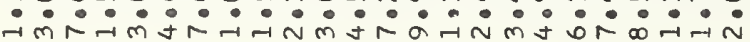

MNN N N

- ar ONm WU

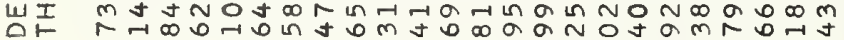
ம்

$\stackrel{5}{I}$

$m \pi a m$ o Nitato

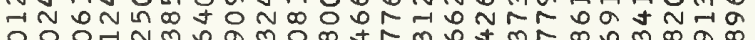

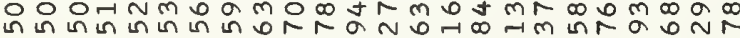

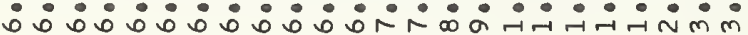

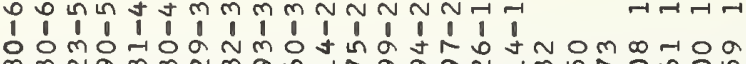

$\neg \sqcap \sqcap+$ $\varangle$ 1 工 u

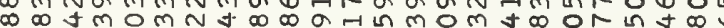

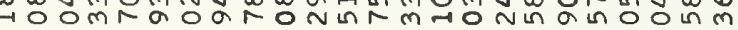

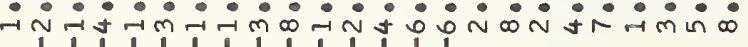
o o o 0 in ํำ

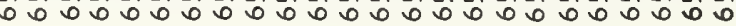

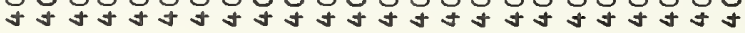

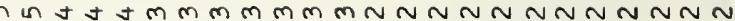

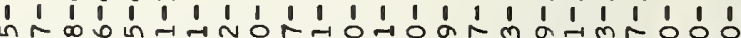

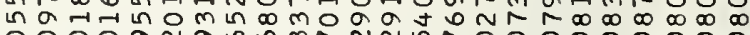

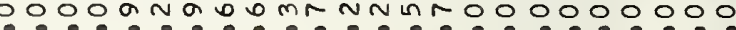
$+\infty N+r-\pi N m$ I

$\infty \infty$ r r r $\begin{array}{lllllllllllllllllllllllllllll}1 & 1 & 0 & 1 & 1 & 1 & 1 & 1 & 1 & 1 & 1 & 1 & 1 & 1 & 1 & 1 & 1 & 1 & 1 & 1 & 1 \\ 0 & 0 & 0 & 0 & 0 & 0 & 0 & 0 & 0 & 0 & 0 & 0 & 0 & 0 & 0 & 0 & 0 & 0 & 0 & 0 & 0 & 0\end{array}$

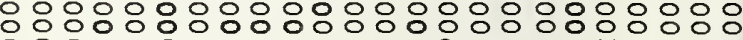

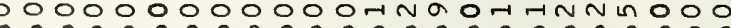

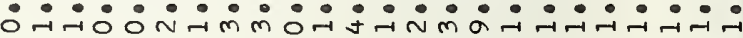

$\rightarrow 1$

$\pi-H-H N N$ N

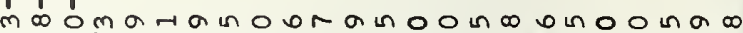

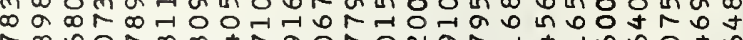
m 00000 in a 0 a

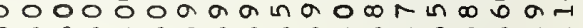
-

$m m N N$ N

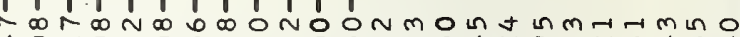

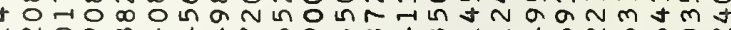

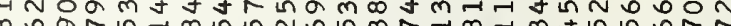

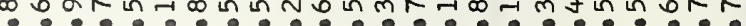

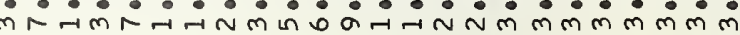

$m \sim N \sim F-1-1$

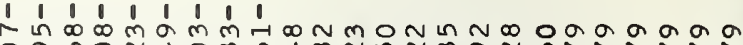

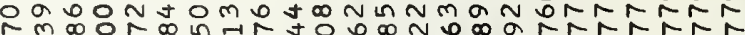
mo

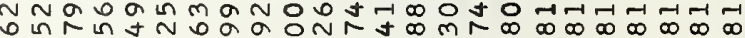

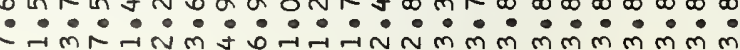

$\pi-1-1$

$-H-H \rightarrow N N N N N$ N

DrómmN oN m $m$ J

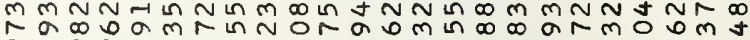

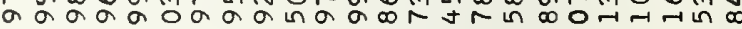

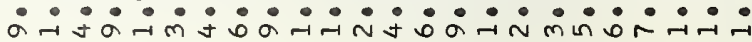

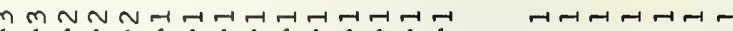

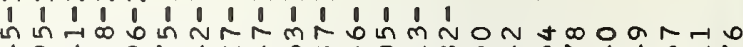

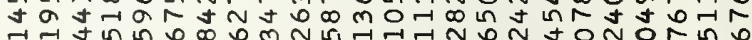

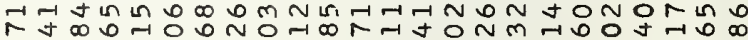

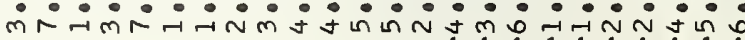
$\begin{array}{llllll}1 & 1 & 1 & 1\end{array}$

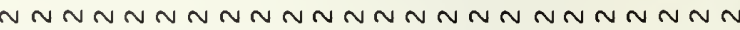

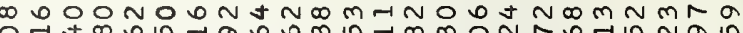

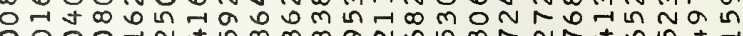
O $000 \pi N$ J $00000000000 \pi n N$ N -1

O 0 b

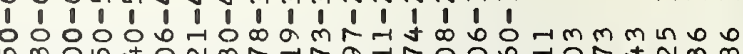
n $m$ O

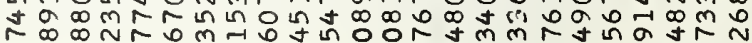

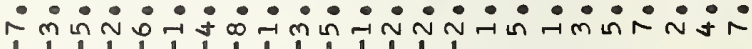
1 1 1 1 1 1 |

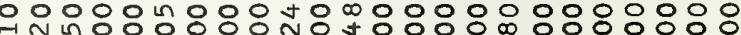
-

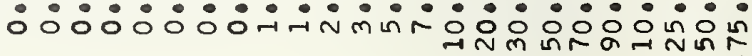
rNm $\mathrm{N}$

000000000000000000000000

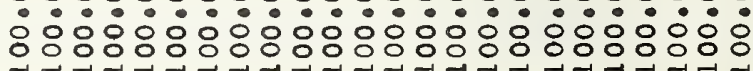

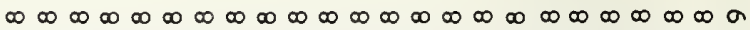

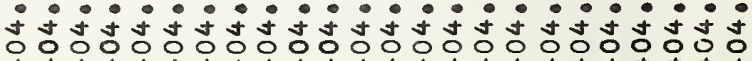

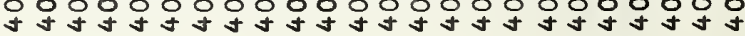


nntattammmmmmmmnNN $\begin{array}{llllllllllllllllllllllll}1 & 1 & 1 & 1 & 1 & 1 & 1 & 1 & 1 & 1 & 1 & 1 & 1 & 1 & 1 & 1 & 1 & 1 & 1 & 1 & 1 & 1 & 1 & 1\end{array}$ ษ๋

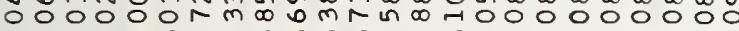

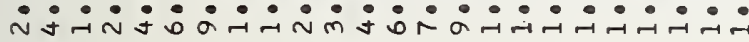

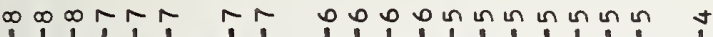

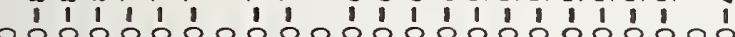
응응응ㅇㅇㅇㅇㅇㅇㅇㅇㅇㅇㅇㅇㅇㅇㅇㅇㅇㅇㅇㅇㅇㅇㅇㅇㅇㅇㅇㅇㅇㅇㅇㅇㅇㅇㅇ

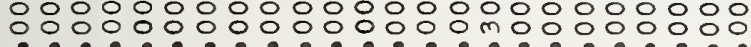

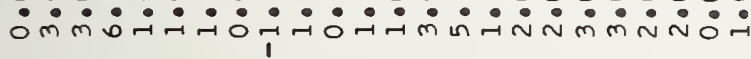

$\because \rightarrow-1+1$

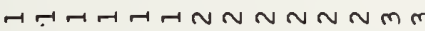

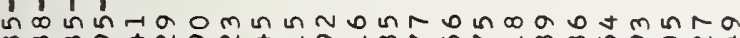

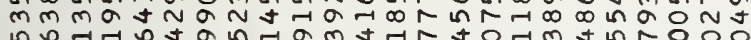

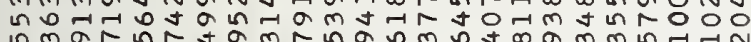

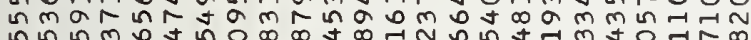

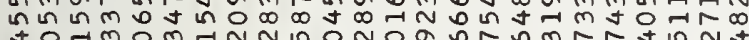

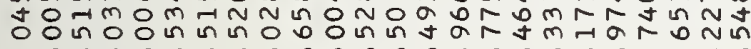
ம்ரும்

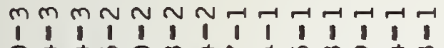

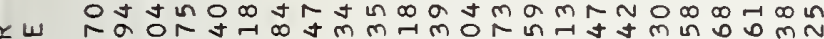

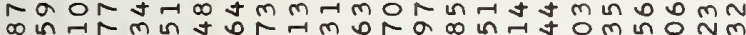

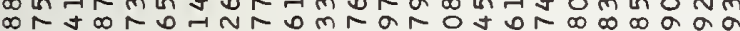

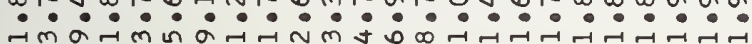

$m m \sim N \sim \neg-1-1-1$

$1,11,1,1,11$,

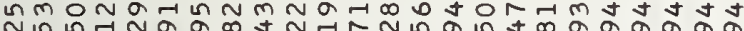

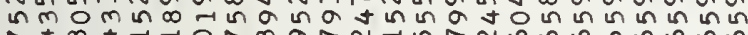

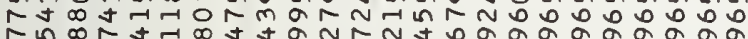

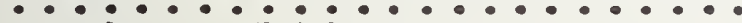

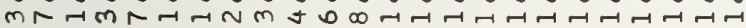

$\operatorname{nnt-14}$

u

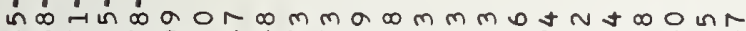
tormRonjtrR⿻ nN $N m$ in in $0 m$ bo 0 in $n$ o ta a $\because \infty \exists a \infty$ ᄂ

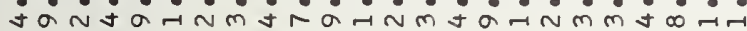

mmmNNNNー

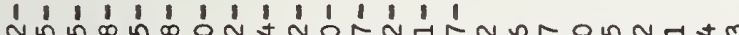

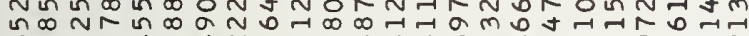
m motiotno n

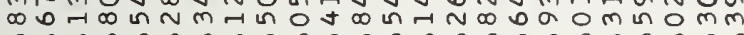
$\infty ? \because \infty$ ?

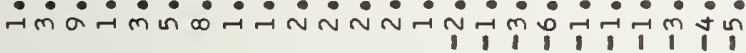

NNNNNNNNNNNNNNNNNNNNNNNN

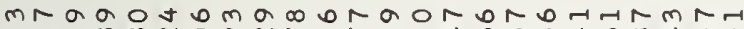

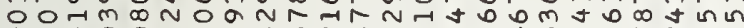

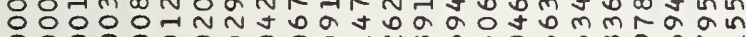

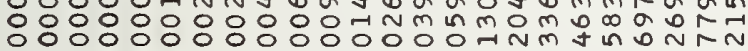
vivivivivivivivivivivivivim

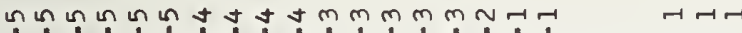

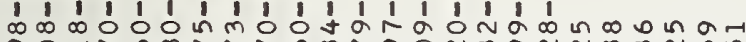

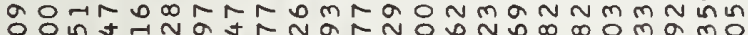
윰ำ

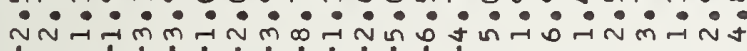
$1,1,1,1,1,1,1,1,1,1,0$

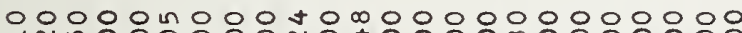

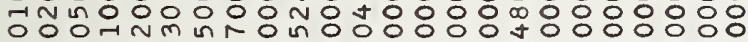

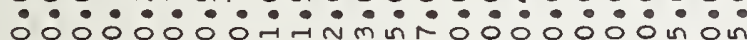
$00000000 \pi-N m$ m

000000000000000000000000

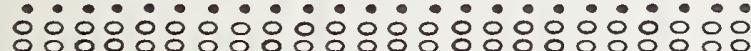
N N N N N N N N N N N N N N N N N N N N N N

$\infty \infty \infty \infty \infty \infty \infty \infty \infty \infty \infty \infty \infty \infty \infty \infty \infty \infty \infty \infty \infty a$

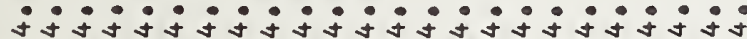

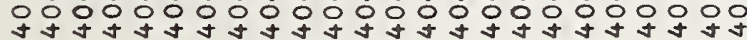

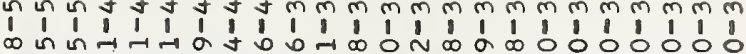

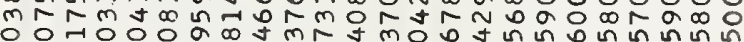

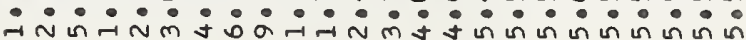

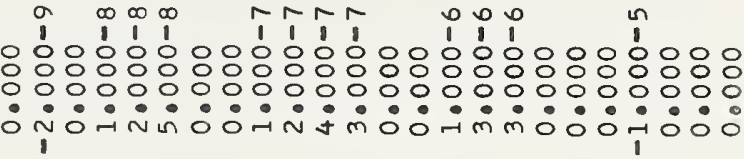

NTrar

NOIn

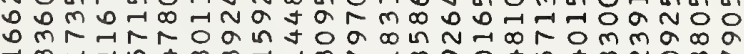

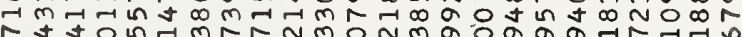
von เก

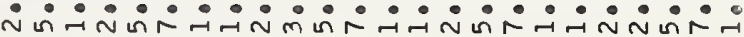

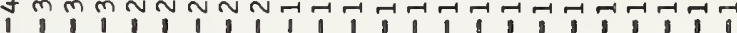

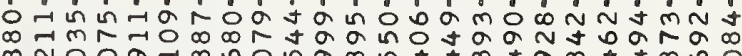

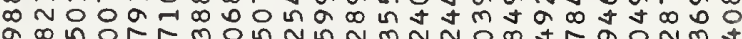

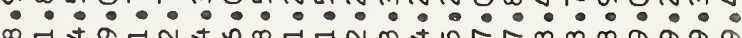

\section{mmm $m$ N}

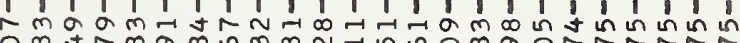

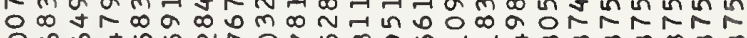
O D U J in N N O

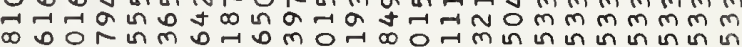

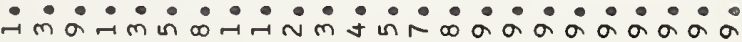

ก

A-4-ThNNNNNN

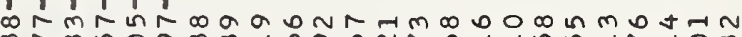

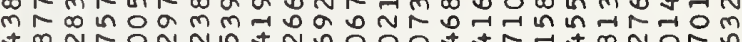
AN $\infty$ N min

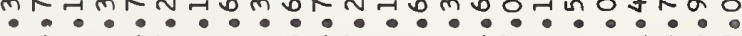

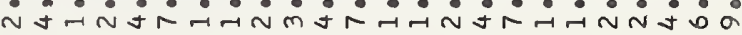

AmmmnNNNNN-THN-1

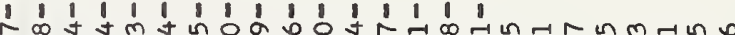

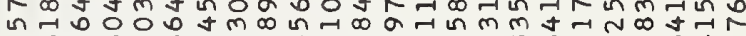
N m $m$ m n

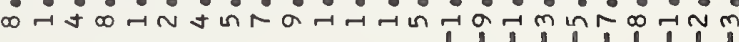

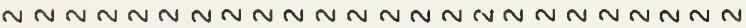
Tmaamaaoun 000 m

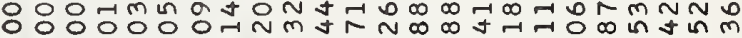
$000000000000 \pi n n$ $0000000000000000-1 \mathrm{~mm} 0.7$

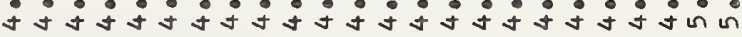

o.

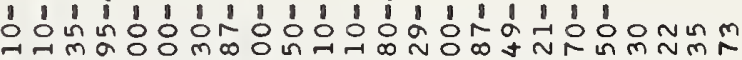

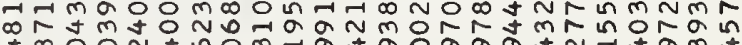

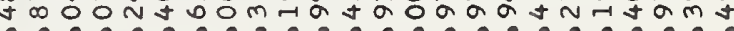

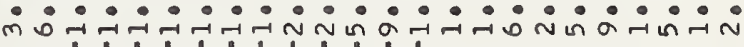

$00000 \mathrm{~m} 000$ t 0 m000000000000 o

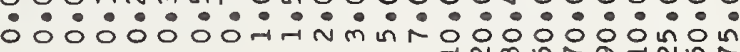
TNmURan

000000000000000000000000

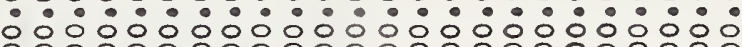

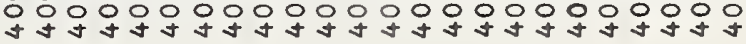

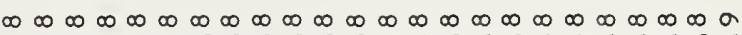

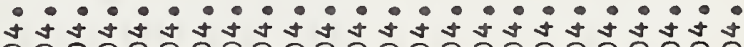

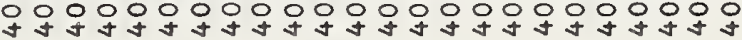




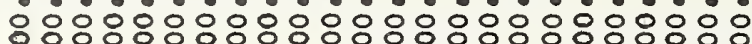

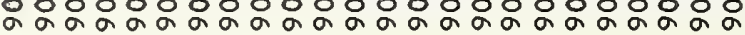

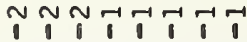

$-1-1-1 \times N \sim \sim 2 N$

mmentma in H D $m$ N ON

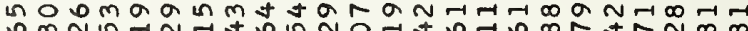

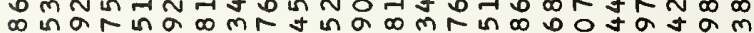

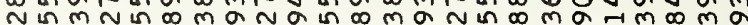

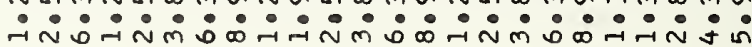

ป

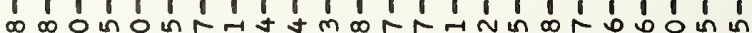
+

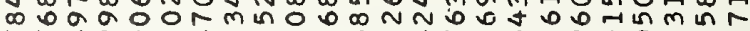
ก

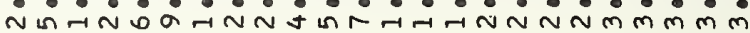

ง $m m \sim N N N N N-1-1-1-1-1-1-1-1-17$

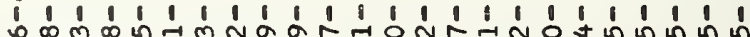
임ำ N $m$ in

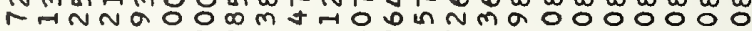
ONOOH

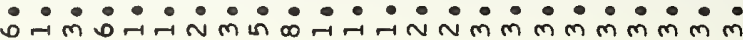

$m \sim N T-1-1)$

HA-

$\sim \infty m \infty$ ó $\infty m$ in $N$ m N ON

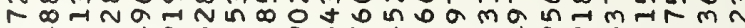

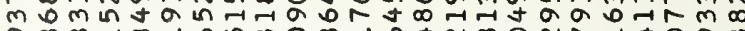

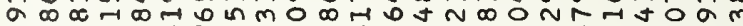

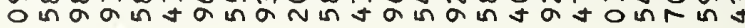

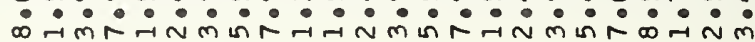

J

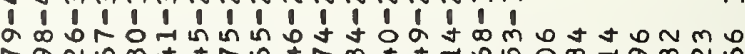
ranomatrotiomsta

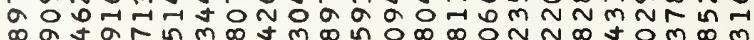
$\infty a+a r$ in $m \infty$ t $m \infty$ in $0 \infty \infty \circ \sim N \infty$ t $0 m \infty m$

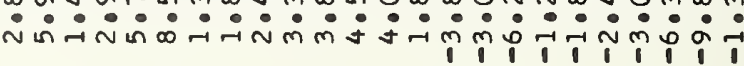
NNNNNNNNNNNNNNNNNNNNNNNN

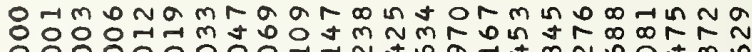
ㅇㅇㅇㅇㅇㅇㅇㅇㅢ 응ㅇㅇㅇㅇㅇㅇㅇㅇㅇㅇㅇㅇㅇㅇㅇㅇㅇ인 áa a a a a a a a a a a a a a a a a

A

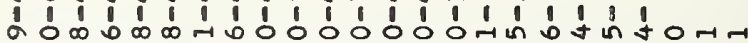

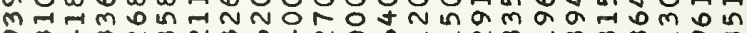
aำ

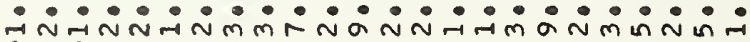

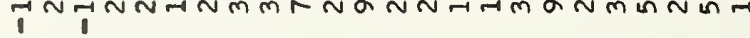

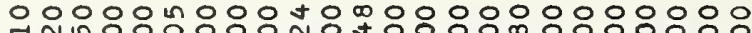

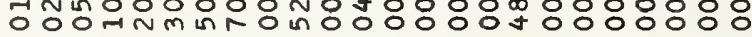

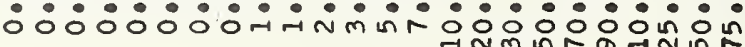

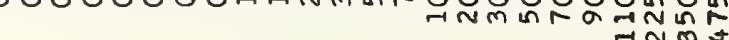


mกNNNNNNNNATH

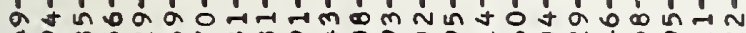

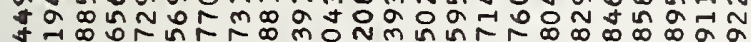

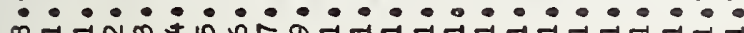

OUn o

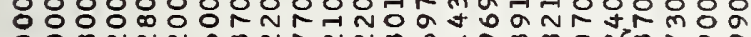
OO

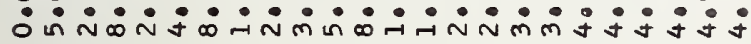

$-\pi-\pi \rightarrow N \sim N N N N N N N N N N N m m m m m m$

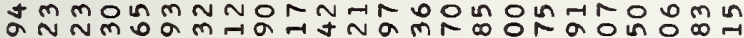

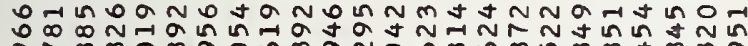
o $T$ in $a$ N ? ? ? ? ? ? ? ?

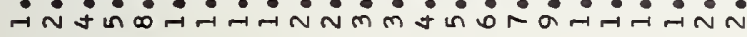

$\overrightarrow{1}$

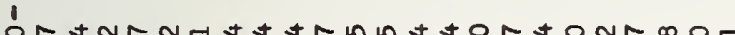

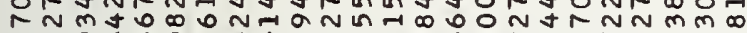

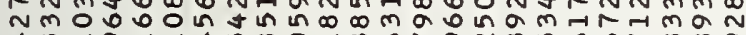

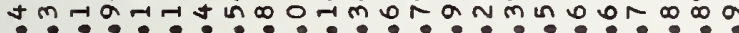
a - NNA

m

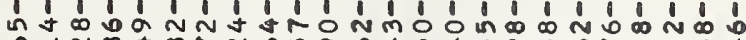

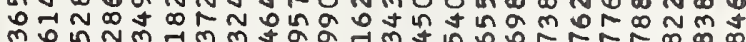
- ?.?! ? ? ? ? ? ? ? ? ஸिंत்

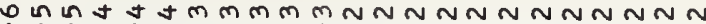

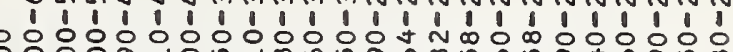

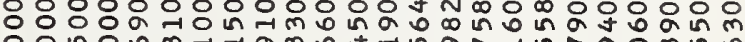
으의.

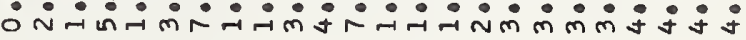

-1 $-1-1 N \mathbb{N}$ -

Nma on $n-1+\infty N R a+m a n \circ 0 \infty a \infty m+n$ $m-n N M N$ N ${ }^{-1} \infty$ in

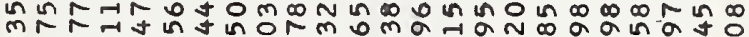

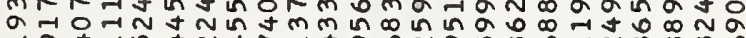

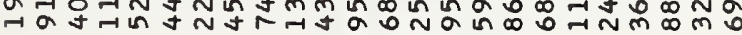

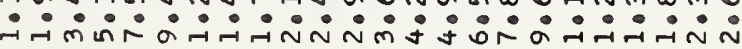

까

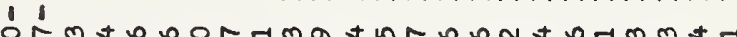
రิm

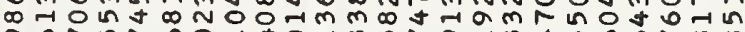
a v n na-nm\&

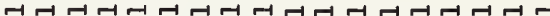

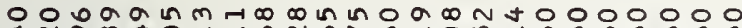

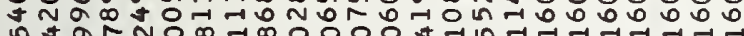

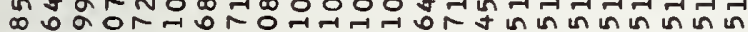

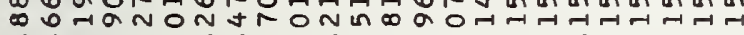

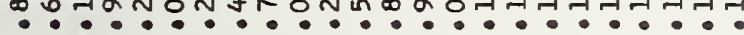

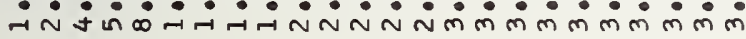

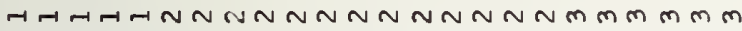

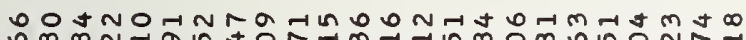

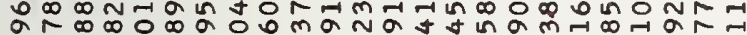

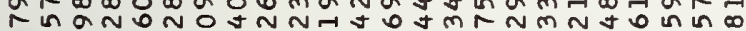

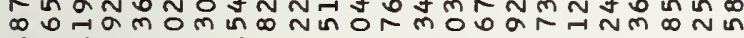

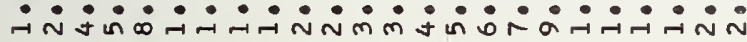

$\ll \ll$

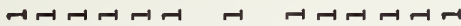

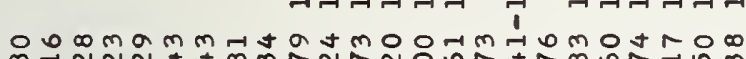
\&

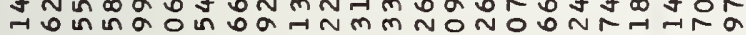
$\because ? ? ?$ ? ? ? ? ?

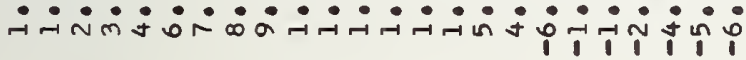

A

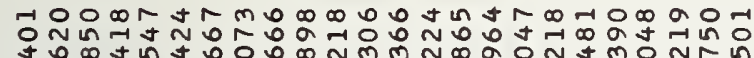

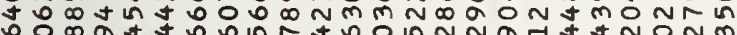

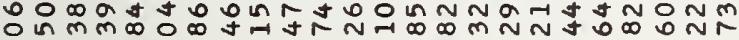

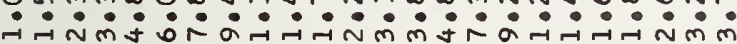

N $N$ N भतम án

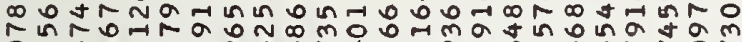
กำ

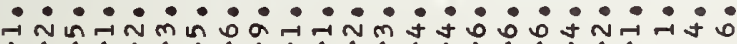
IN

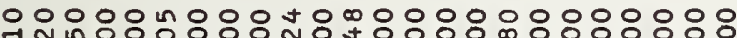

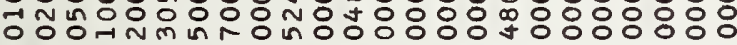

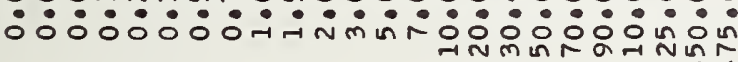
$\rightarrow N m y$

000000000000000000000000 í

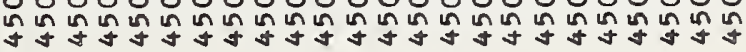

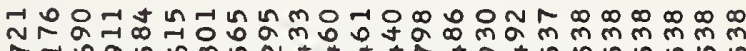

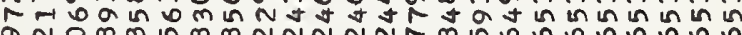

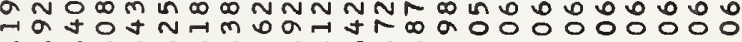

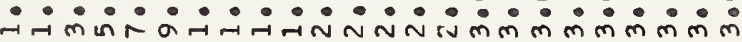

MOO 0 - $N$ N min

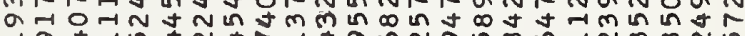
$\because 9.7$ ก

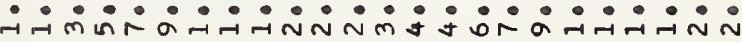

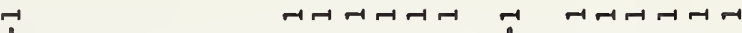
an-1-10\%a a N a

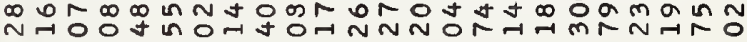

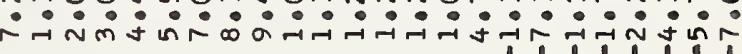

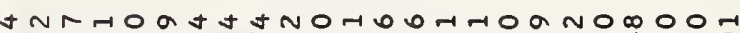

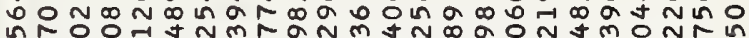

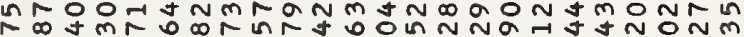

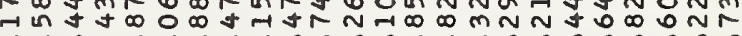

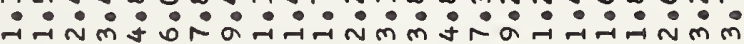

$m \sim N N-1-1-1-1$

$\rightarrow-1-1$

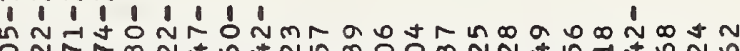

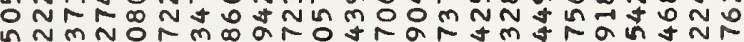
ñ $N$ m

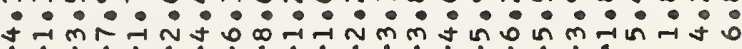

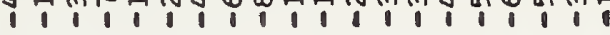

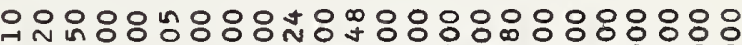

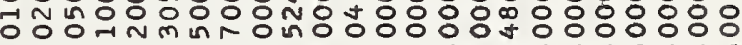

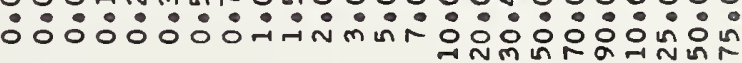

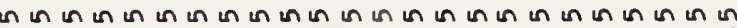

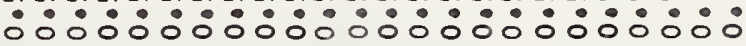




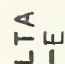

㟧品

.

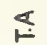

$\vec{u}^{\alpha}$

mMNNNNNNNNNATH

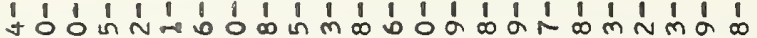
in 0 in mंம்-mm

ด $\begin{array}{lllllllllllllllllllllll}1 & 1 & 1 & 1 & 1 & 1 & 1 & 1 & 1 & 1 & 1 & 1 & 1 & 1 & 1 & 1 & 1 & 1 & 1 & 1 & 1 & 1 & 1 \\ 0 & 0 & 0 & 0 & 0 & 0 & 0 & 0 & 0 & 0 & 0 & 0 & 0 & n & 0 & n & 7 & 0 & 0 & 0 & 0 & 0 & 0\end{array}$

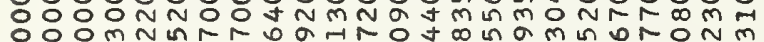
inrmaínian்

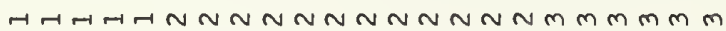

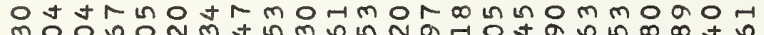

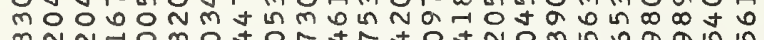
-

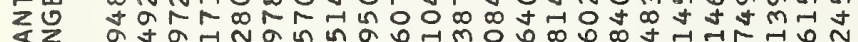

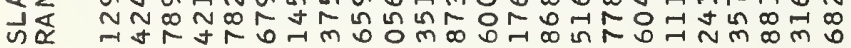

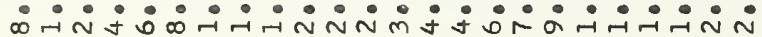

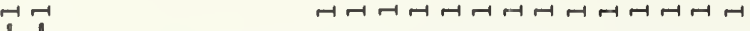

If $+m$ in $\infty$ int

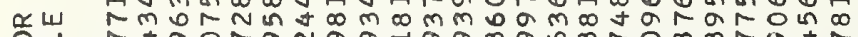

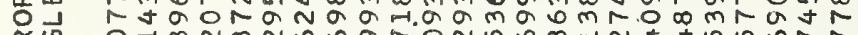
$\frac{\alpha}{\alpha} \mathcal{O}$ ш

1

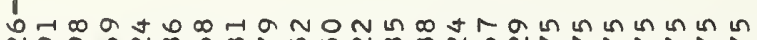

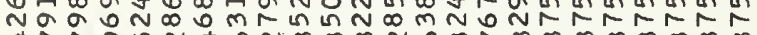

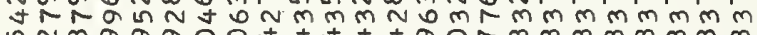

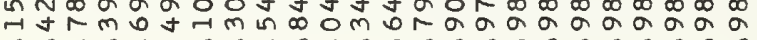
- . . . . . . . . . . mañW

H- $-1-N N N N N N N N N N N$ N

N-HOR

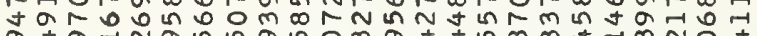

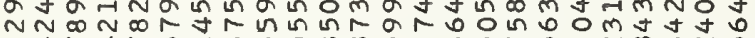
N

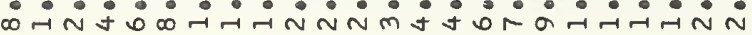

$\ll \ll$

11

$\sqcap \sqcap-\sqcap-$

-

UI

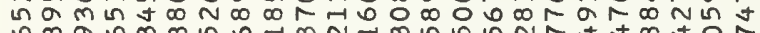

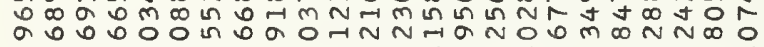

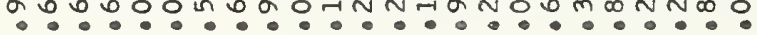

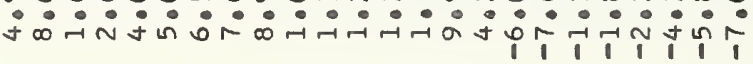

H- - H - H

$\underset{⿱ ㇒}{\mathbb{I}}$

$\infty$ tamanr th $m$ t 0 o 0 m + $0^{\infty}$ in

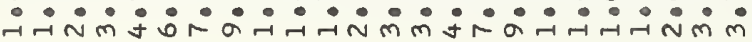

$m$ m N

$\rightarrow-1$

NI

$\varangle$

WI

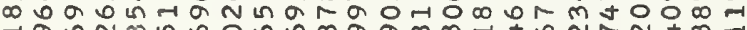

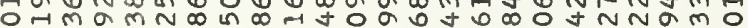
Nin் $\begin{array}{llll}1 & 1\end{array}$

o o o o

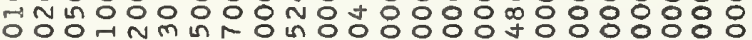
O I $\stackrel{\leftarrow}{E}$

000000000000000000000000 $u$
$\frac{1}{0}$
$\frac{1}{2}$

000000000000000000000000 Lz

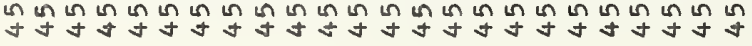

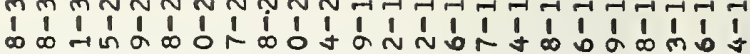

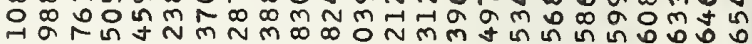

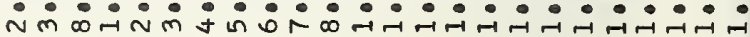

Nomm $\begin{array}{lllllllllllllllllllllll}1 & 1 & 1 & 1 & 1 & 1 & 1 & 1 & 1 & 1 & 1 & 1 & 1 & 1 & 1 & 1 & 1 & 1 & 1 & 1 & 1 & 1 & 1 \\ 0 & 0 & 0 & 0 & 0 & 0 & 0 & 0 & 0 & 0 & 0 & 0 & m & 1 & N & 0 & 0 & 0 & 0 & 0 & 0 & 0 & 0\end{array}$

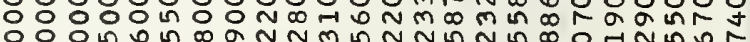

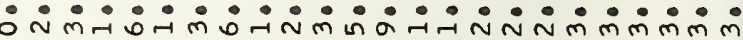

H-H NNNNNNNNNNNNmmmmmm

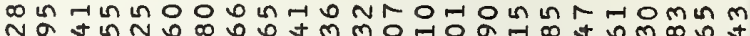

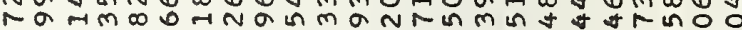

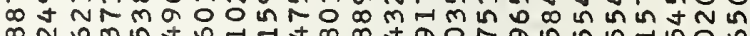

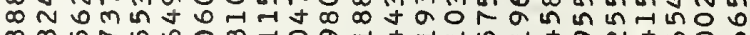

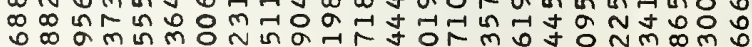

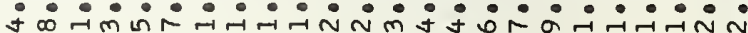

1

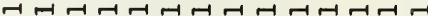

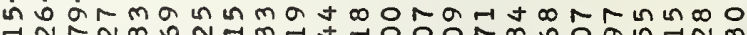
in NN $N$ N N

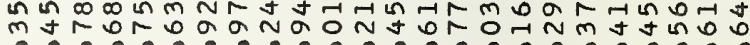

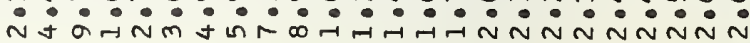

$\sqcap \sqcap$

L

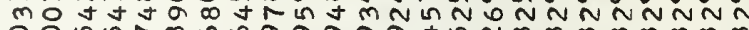

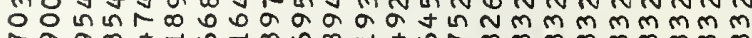

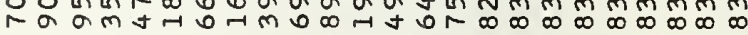

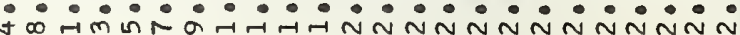

Hr $-1 N N N N N N N N N N N N m m m m$ In 0 O G

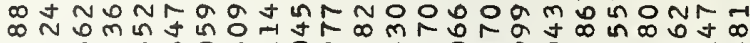

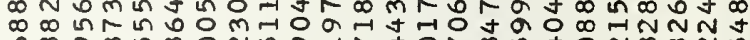

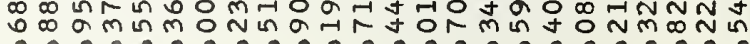

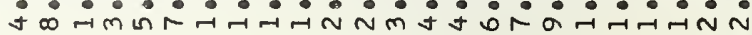

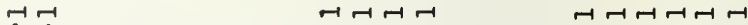

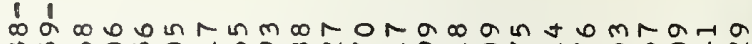
$m$ L 00 L 0 O 0 a $N$ N

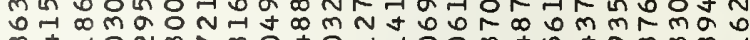

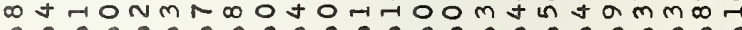

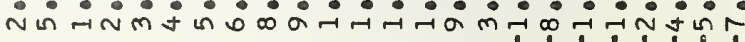
HคH

NNROOJNNNGM

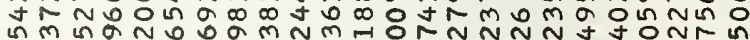

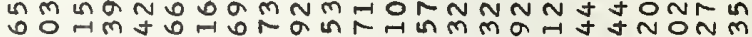

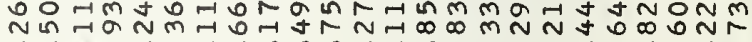
NंNm

\m NNNHHH

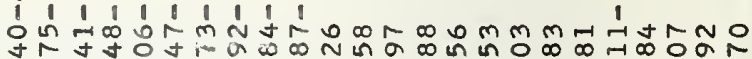

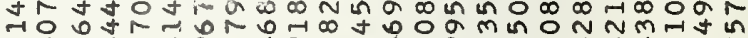

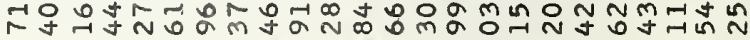
-

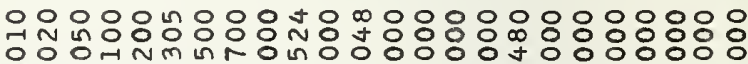

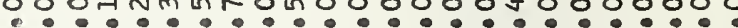

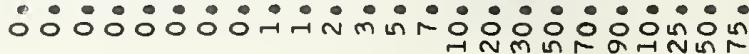

000000000000000000000000

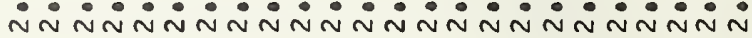

000000000000000000000000

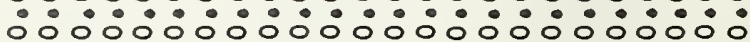
in in in m in in in in in in in in in in in in in

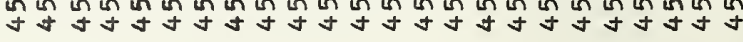


mmmm $m$ m

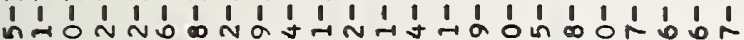
مR

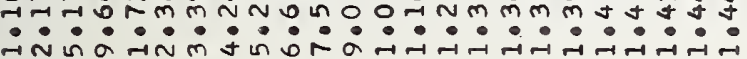
N D nInatammmmmnNNNNNNNNN

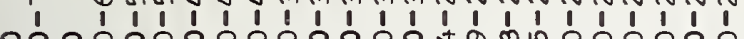

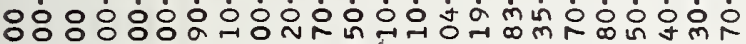
o 00 nt

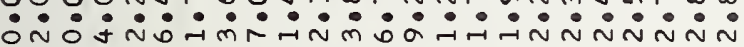

П- П-

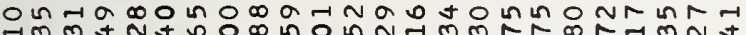
- $m m$ m

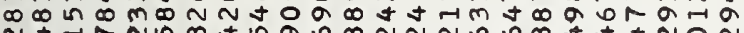
N寸

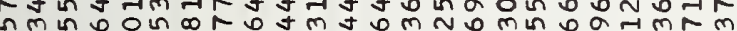

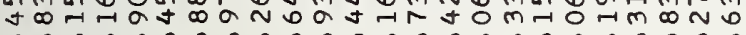

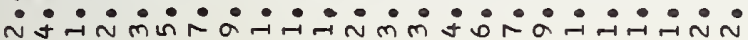

T1

mmmo on D n

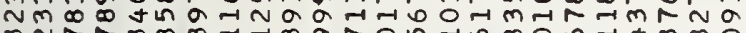

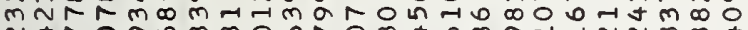
ขป

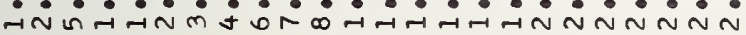

군

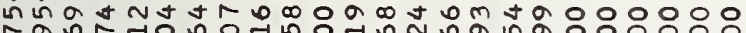

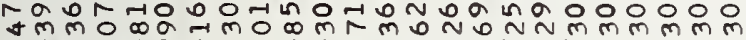
0 in $n$ mos

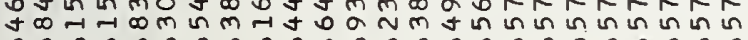
Nษ - $-\pi-H N N N N N N N N N N m m m m m m$

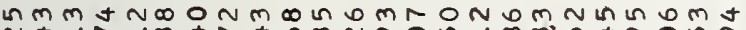

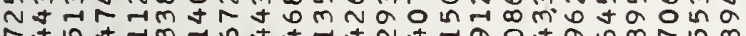

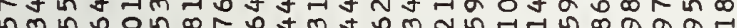
nn

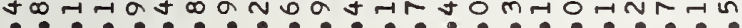

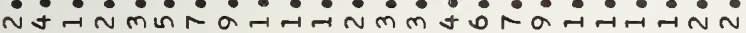

$\rightarrow-17$

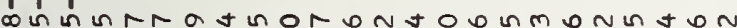

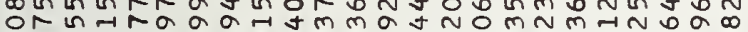

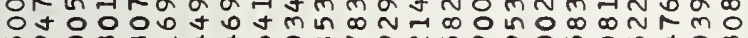

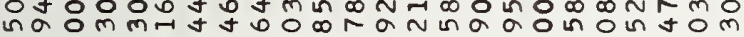
แ? ? m!?

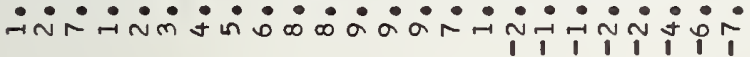

-

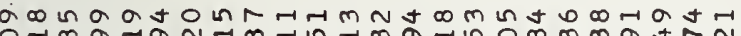

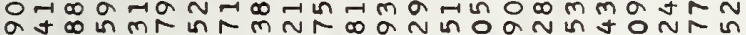
ma mN

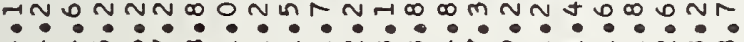

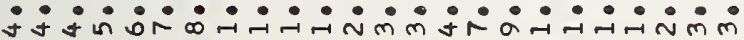

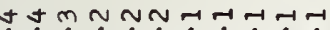

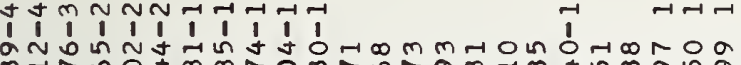

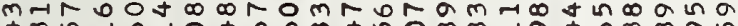

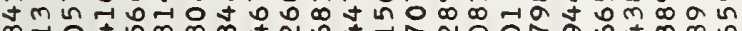
? í,

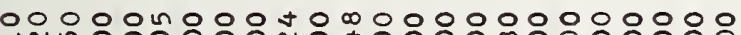

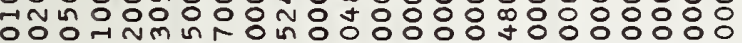

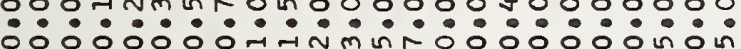

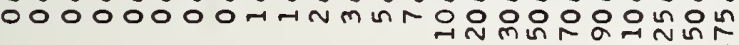

000000000000000000000000

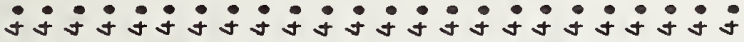

000000000000000000000000

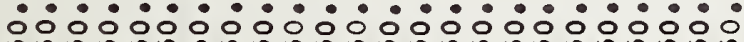
แn แก

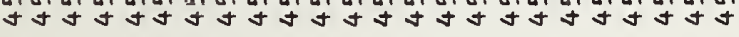

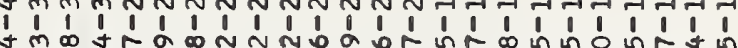

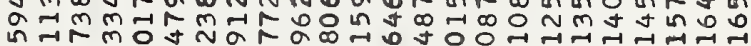

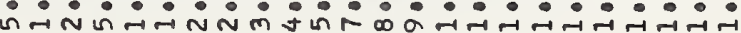

TommatammmmmnnnNmnnnN

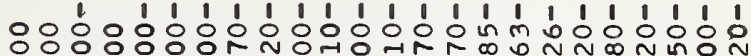

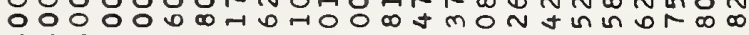

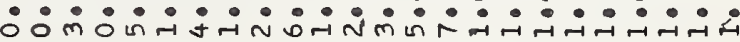

- $-1-H-N N N N N$ N

Nank

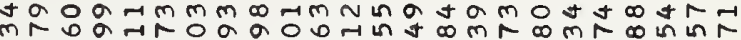
Un $N$ in

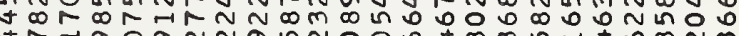
ง

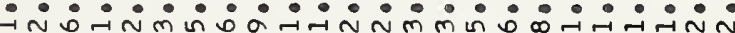

$i \mathfrak{1}+1$

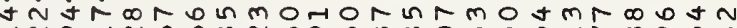
- NaTND

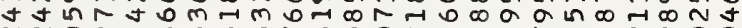

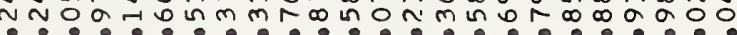

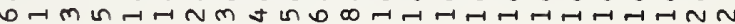

$17-1$

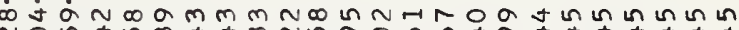
$n$ om

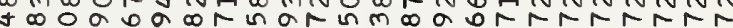
NATnNajmon

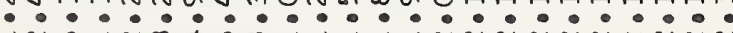

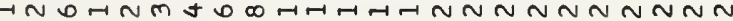

$-1-1-1, N N N$ N N N N

a a mantarta + -1 in $m$ ONR ง Nป -

$\operatorname{11}-1+1$

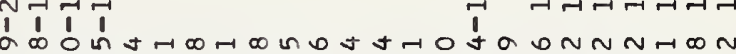

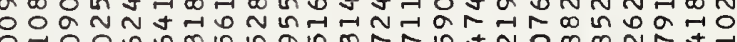

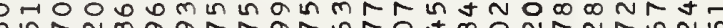

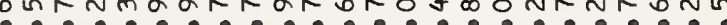

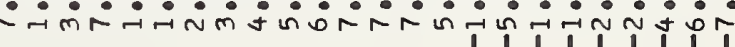

-

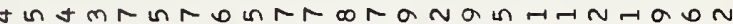
t In NaDaamsonOm tor

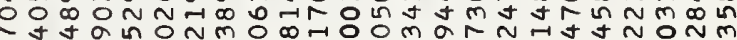
현

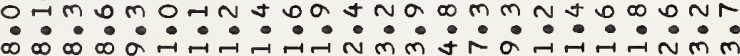

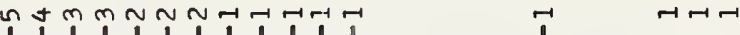

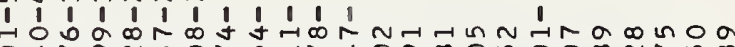
trandor $n$ Th

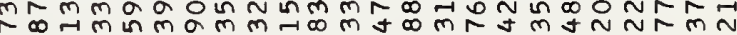

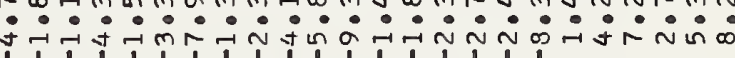

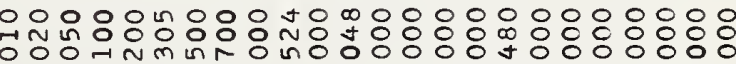

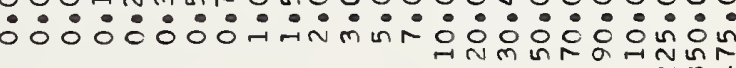

000000000000000000000000

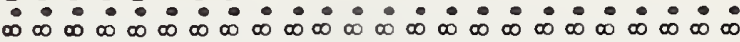

000000000000000000000000

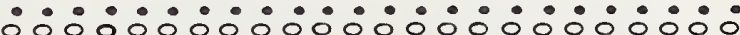
nn in in

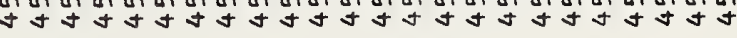


$\varangle$

$\stackrel{5}{u} \alpha$

ᄂu

ज)

$\propto w$

$\stackrel{\alpha}{\alpha} \frac{}{\alpha}$

$\frac{\alpha}{w}<$

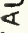

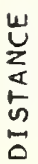

\&《 1171

$\vdash$

岂㞬

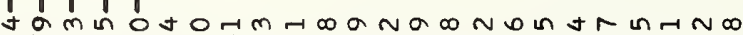

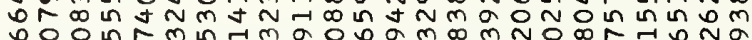

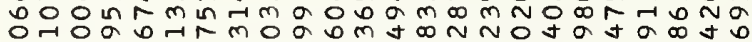

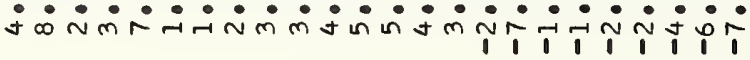

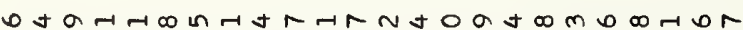

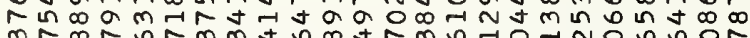

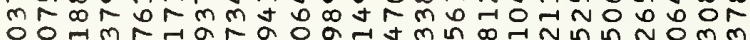

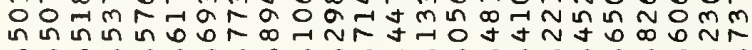

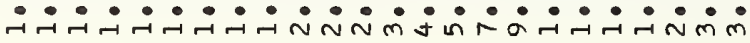

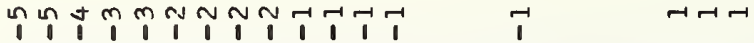

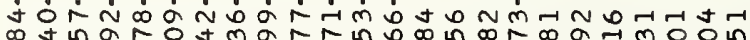

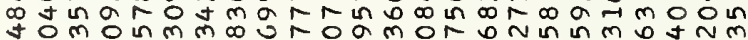

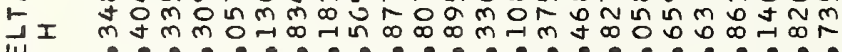
崖

$\frac{5}{ \pm}$

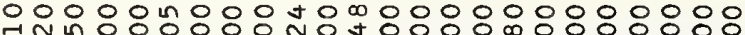
o o onnm nno not

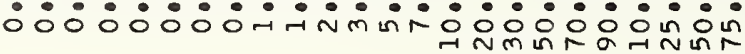
$\rightarrow \sim m a$

世

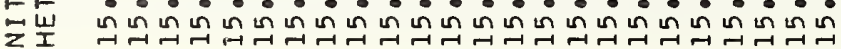

J J con

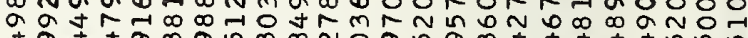
ta tamanm $n$ o

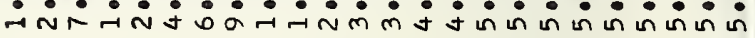

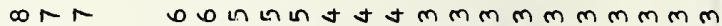

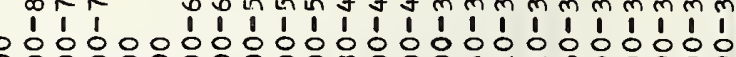

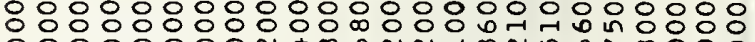

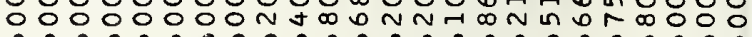

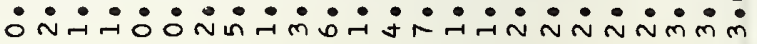

it

II

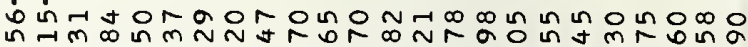
o ta n 0 on $N$ N 춰ำ N mojoñom $m$ mo:

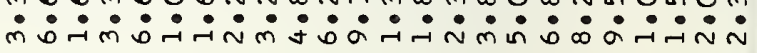

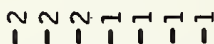

$4-4-4-1$

I I I

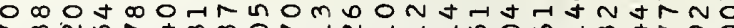
om $m$ m

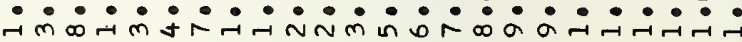

$\operatorname{nat}+1-1$ $4-1-4-1-1-1-1$

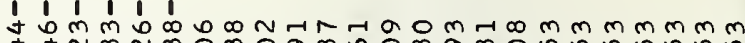

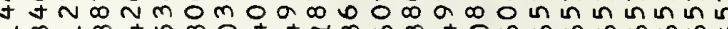
t m

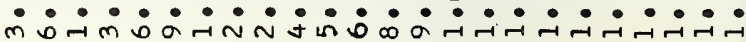

1)

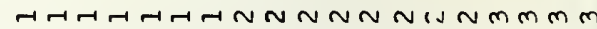

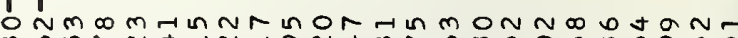

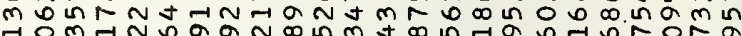

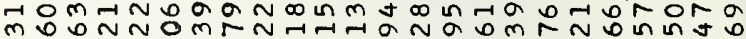
$m$ mo 0 o

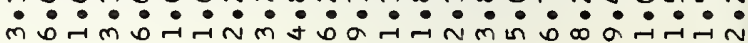

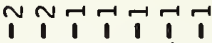

$4-4 \pi-4 t$

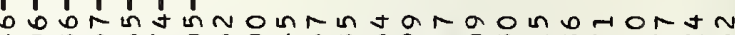

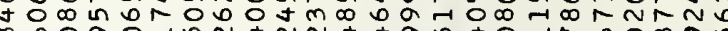

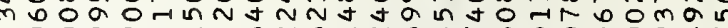

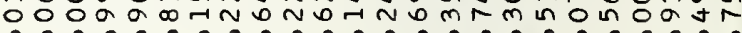

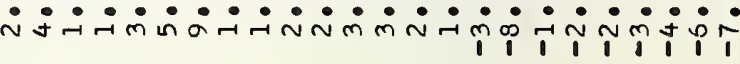

orambaga ba Drat

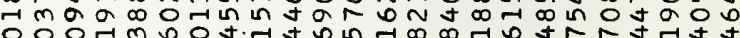

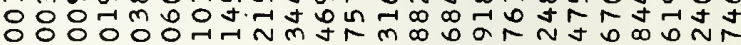
$\dot{m} \dot{m} \dot{m} \dot{m} \dot{m} \dot{m} \dot{m} \dot{m} \dot{j} \dot{i} \dot{i} \dot{\sigma} \dot{-} \dot{-} \dot{-1} \dot{\sim} \dot{m}$

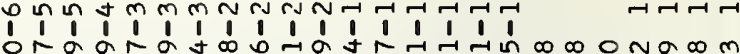

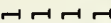
ambarbyam $n-\pi N \infty$ or

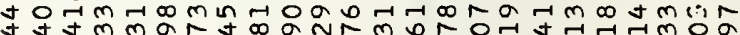

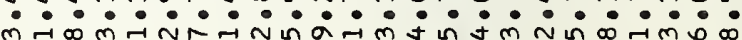
m

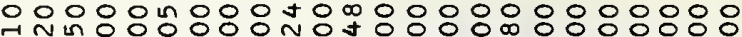

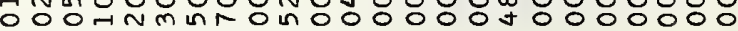

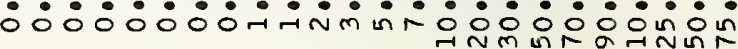
$\rightarrow N m$ J

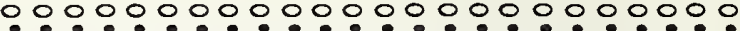

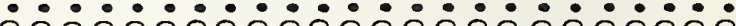

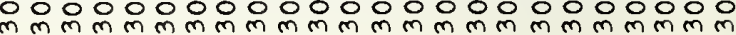

000000000000000000000000

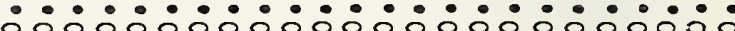
in in in in un in un in in in in in in in un un in in in in in un un

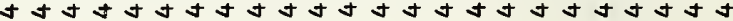




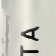

5

岁

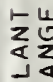

$\vec{n} \approx$

뚠

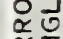

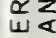

$\frac{2}{4}$

NNNTH-THT

Í

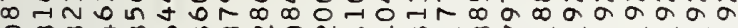

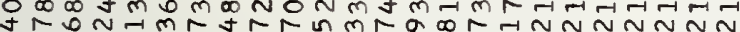

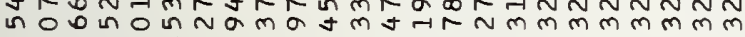

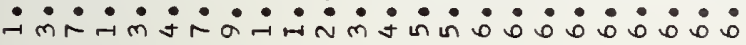

$\overrightarrow{1} \overrightarrow{1}-\overrightarrow{1}$

HAH-H-NNN INNNNmmm

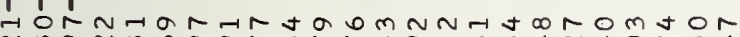
N min

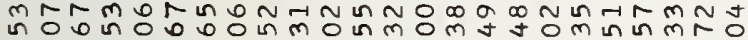

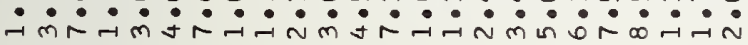

$m \sim n N-1+1-1+1$

H

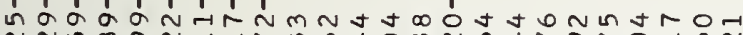
N N

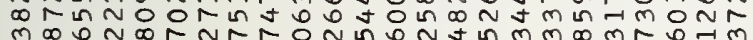

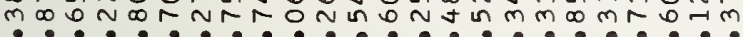

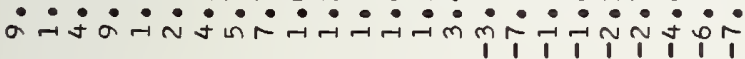

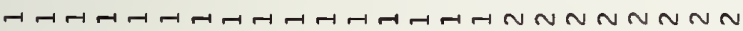

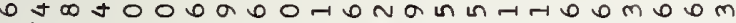

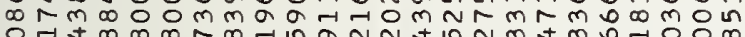
O t O이에 un in un un in in

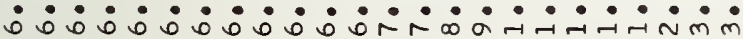

tom not

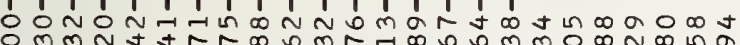

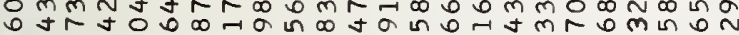

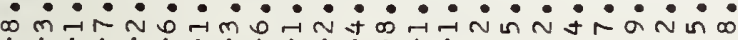
$1,1111111111,11111$

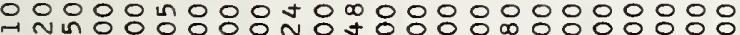
$000-n m$ m non

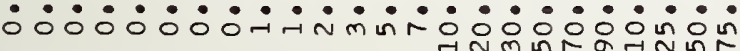
$\rightarrow N m \in \mathbb{N}$

000000000000000000000000 in in in in in in in in in in ดु

000000000000000000000000

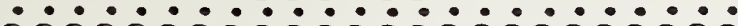
00000000000000000000000

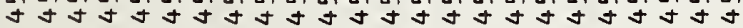

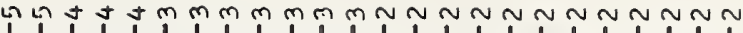
IT

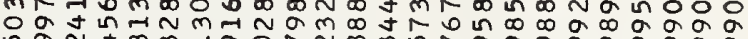
ก a v

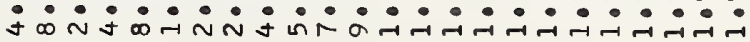

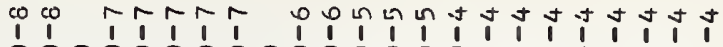

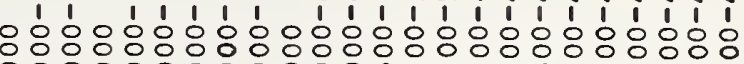

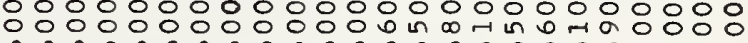

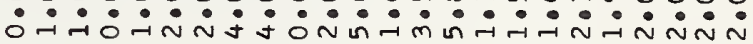

HTH

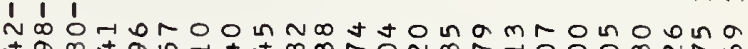

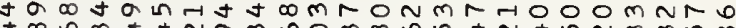
t $\infty 0 m$ t

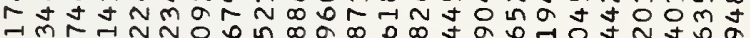

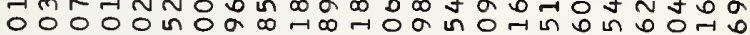
응ㅇㅇㅇ응ㅇㅇㅇㅖ

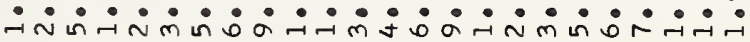

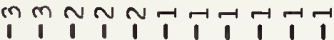

nom 0 L

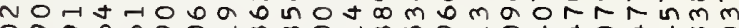

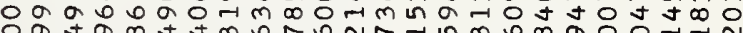

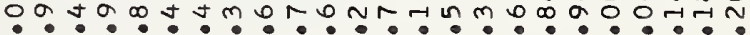
ம் $\dot{\sim} \dot{j} \dot{\sigma} \dot{\sim} \dot{\sim} \dot{j} \dot{i} \dot{\boldsymbol{r}} \dot{i} \dot{\sim} \dot{\sim} \dot{m} \dot{m} \dot{j} \dot{j} \dot{j} \dot{j} \dot{j}$

$m \sim N \sim r-1 \rightarrow r-1$

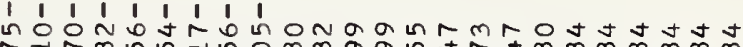

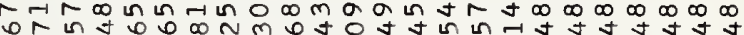
ắ

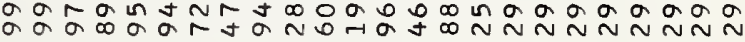

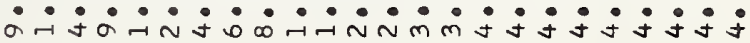

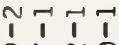

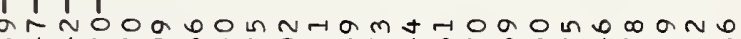

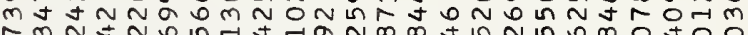
กั

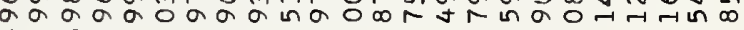

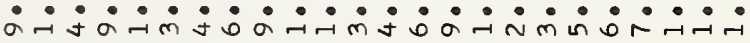

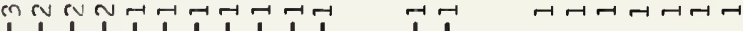

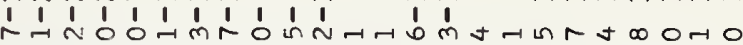

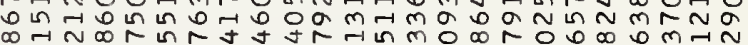

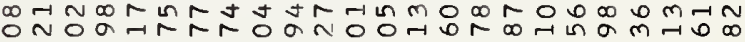

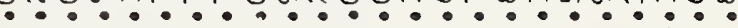

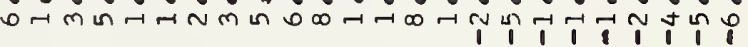

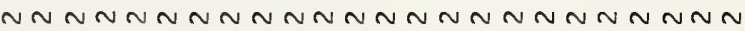

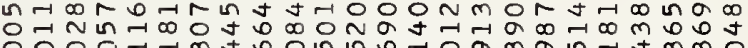

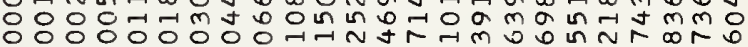

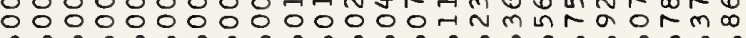

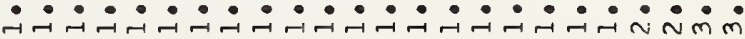

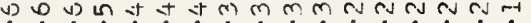

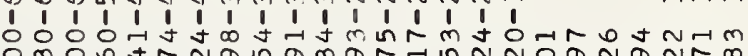
O mobjr

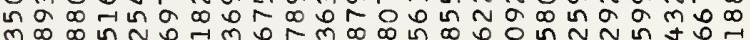

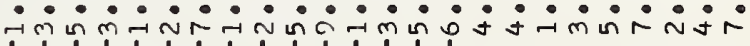
$1,1,1,1,1,1,1111$

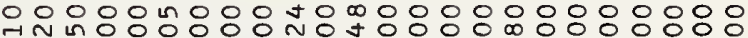

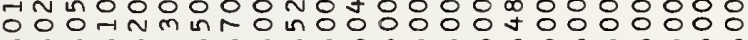

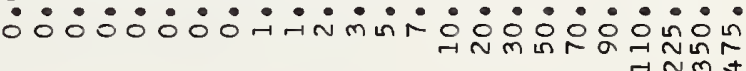

000000000000000000000000

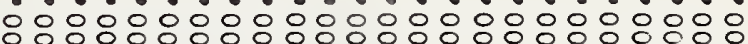

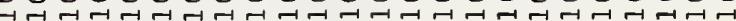

000000000000000000000000

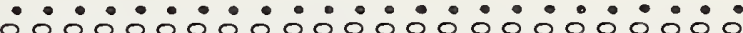
un un un un un un un un un un un un un un un un un un un un un un

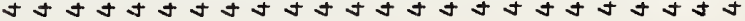




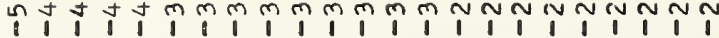

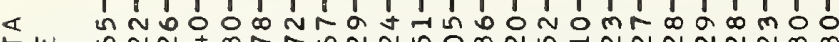

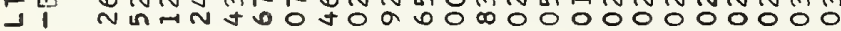

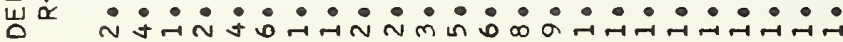

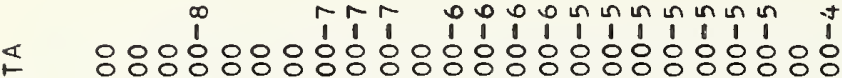

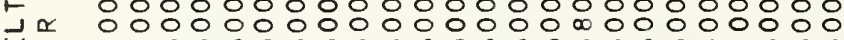 u}

$\hat{1}$

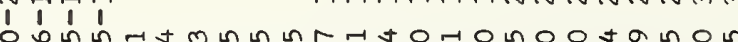

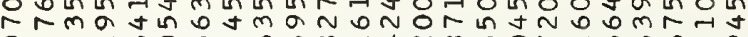

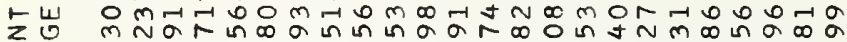

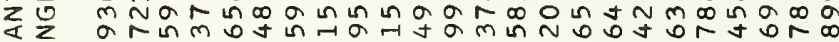
ज选

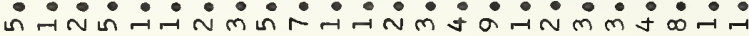

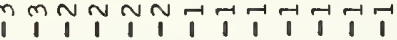

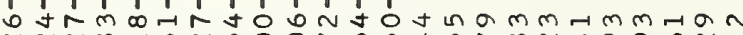

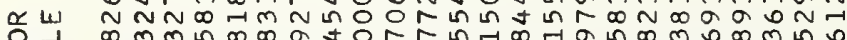

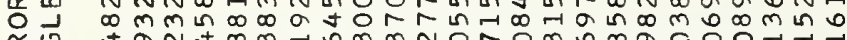

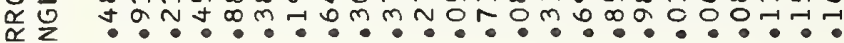
崖

miñ

o O D In

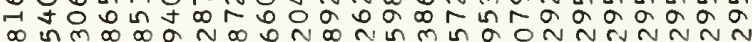
$\frac{2}{4}$

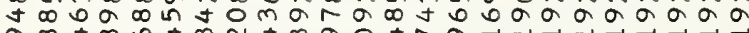

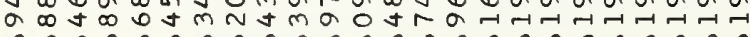

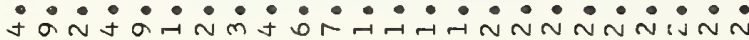

NTH

岁

$\infty 0 \pi$ in $\infty$ in $n 0 m b \infty \pm+\infty \infty \infty N+N+a r \infty$ o

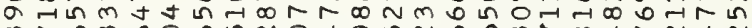

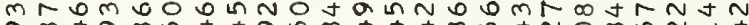
$a_{\infty}$ ta $\infty$ in tyan

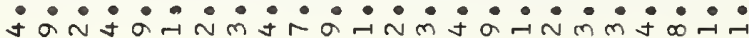

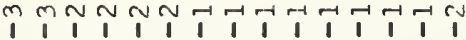

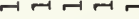

$\varangle \varangle$

w

岁至

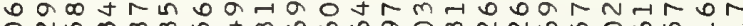
o N n m $\infty m$ in न1

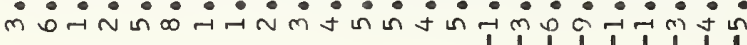

NNNNNNNNNNNNNNNNNNNNNNNN

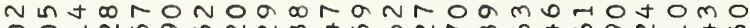

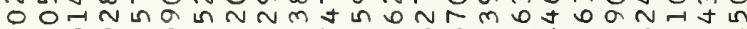

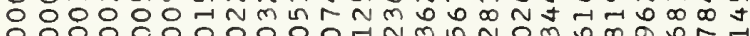

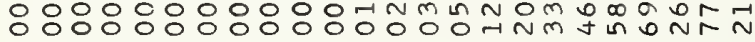

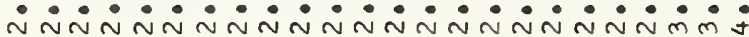

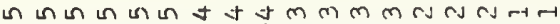

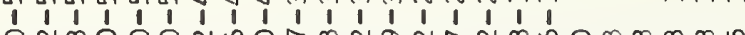

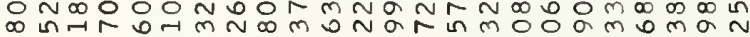
L $\quad$ r JI 岁 $1+1+1$

00000 in 000 tomo00000000000

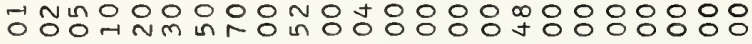

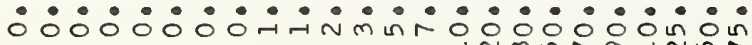
O Oட

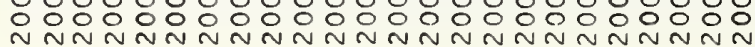

山 $\stackrel{\leftarrow}{\alpha}=$ 000000000000000000000000 z O00000000000000000000000

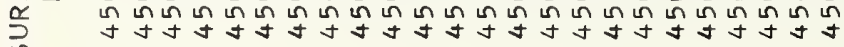

m n

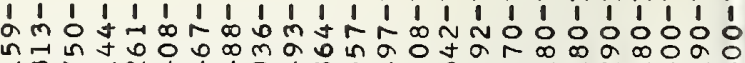
mm - $\dot{N}$ in

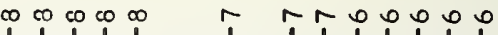
in in o l

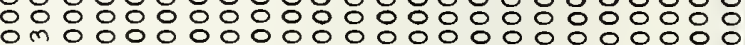

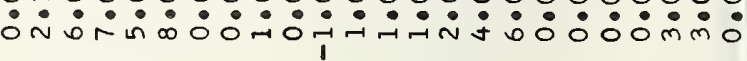
NTH H-HNin

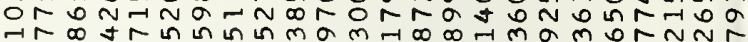

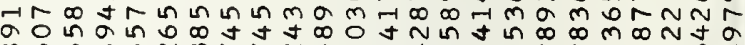

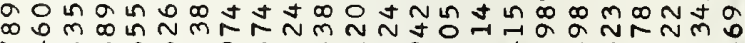
约

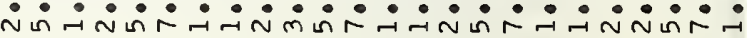

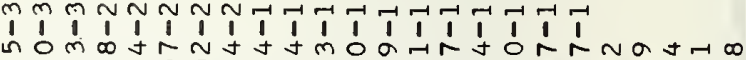

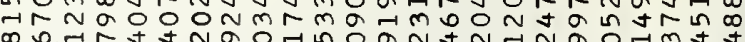

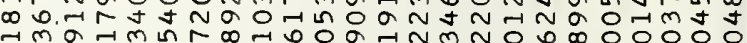

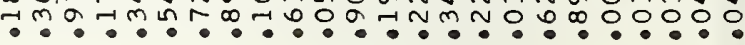
-N

mm NNN

$\begin{array}{llllllllllllll}1 & 1 & 1 & 1 & 1 & 1 & 1 & 1 & 1 & 1 & 1 & 1 & 1 & 1\end{array}$

† Na 0 in $r m \infty y+a n m w b m y+a r g i n j 000000$

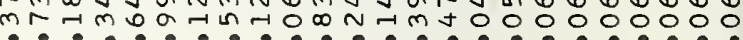

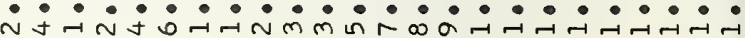

$\hat{1} \mathfrak{1} \overrightarrow{1} \overrightarrow{1}$

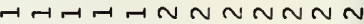

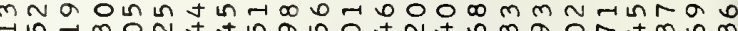

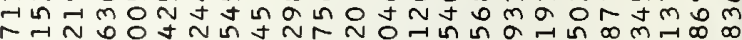

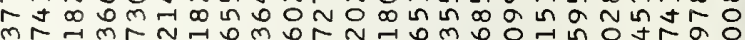
mト

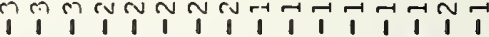

rA

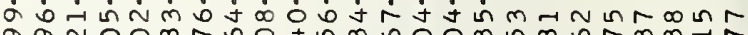

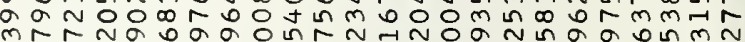

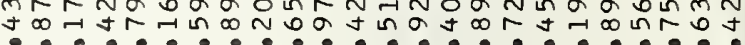

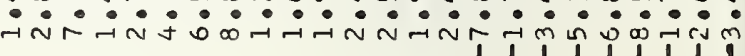
NNNNNNNNNNNNNNNNNNNNNNNN

HNomrmm n 이으의. Oㅇㅇㅇㅇㅇㅇㅇㅇㅇㅇㅇ음 $0000000000000000 \pi-n m m i o t$

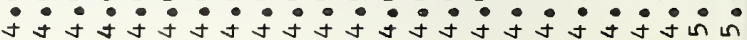

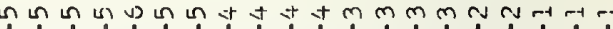

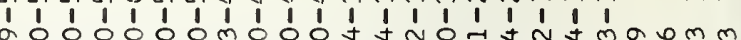

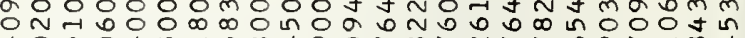

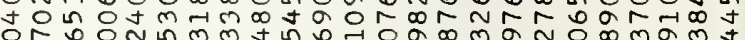

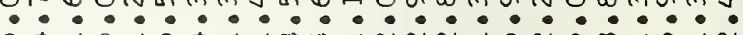
ind

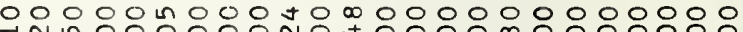

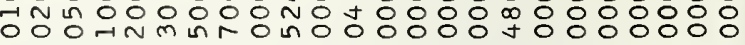

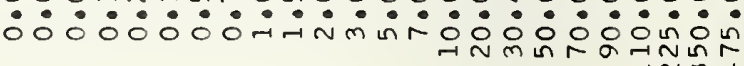
rNm

000000000000000000000000

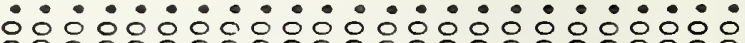

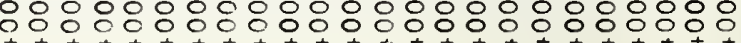

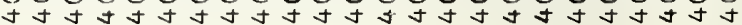

000000000000000000000000

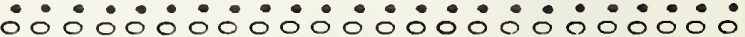

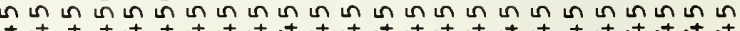




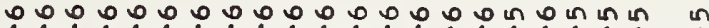
$\stackrel{\frac{1}{b}}{\frac{1}{b}} \alpha$

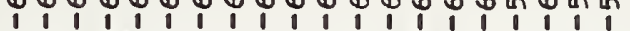

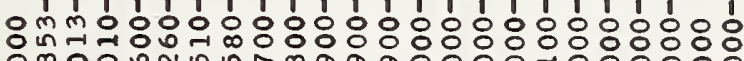

ono o on

On்:

N $N$ N

Am-

$\infty-1$ in $n$ o in 0 mon

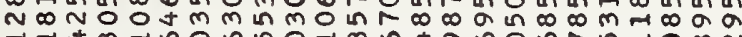

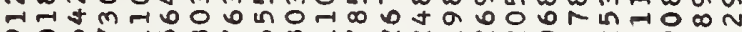
a O OR I $\infty$ in

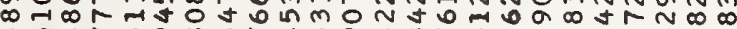

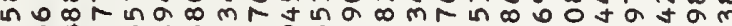
$v \backsim m \sim \backsim \infty m a v \alpha$ in $m a n$ in $\infty m a n m \infty m a$

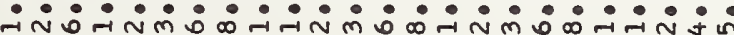

I

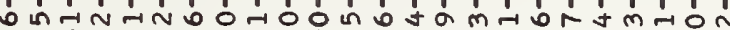
in 00 in $a$ O

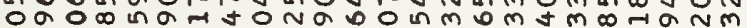

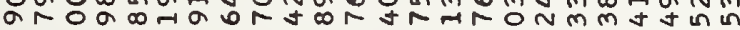
iिi

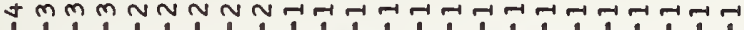

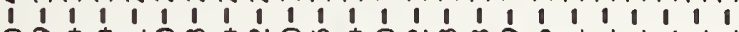

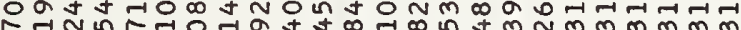
a oNo 000 o in 000 int 0 \% $\alpha$ in $\alpha \infty$ in $m R-1-10$ N

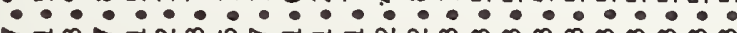

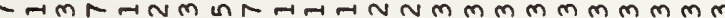

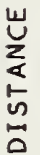

$m \sim N-1-1-1-1$

H

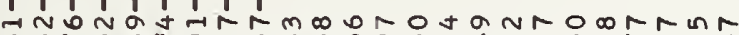
o O in on O

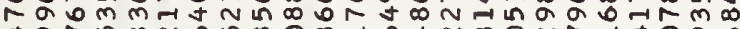

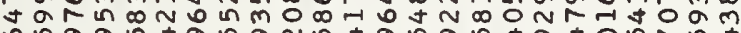

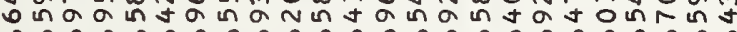

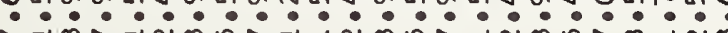

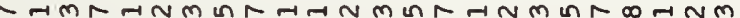

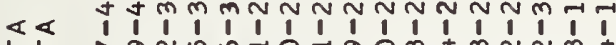

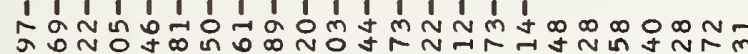

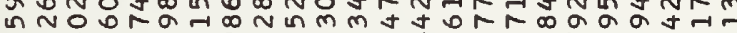
a 0 个m

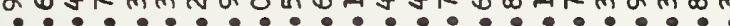

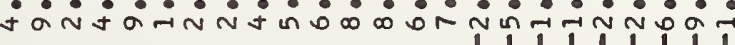
NNNNNNNNNNNNNNNNNNNNNNNN

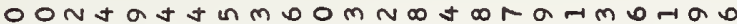

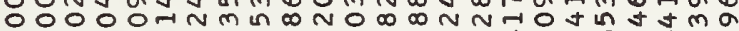

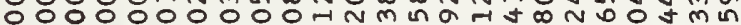
$000000000000000 \mathrm{~m}$ in $\infty 0 \mathrm{~m} 00 \mathrm{~m}$ ○

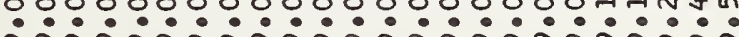

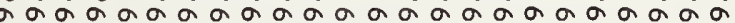

䅧

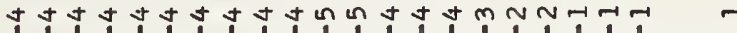

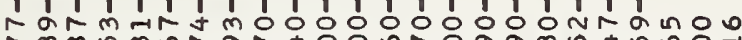
r Nim ? ? ? ? ? ? ? ? ? ? ?

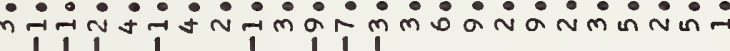
- o o o on o o t o mo o o o o o o o o o

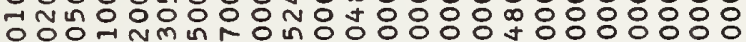

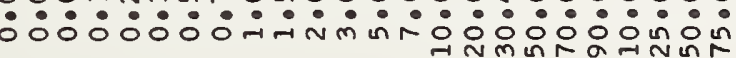

000000000000000000000000

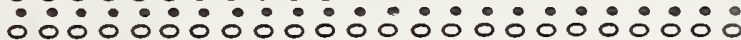

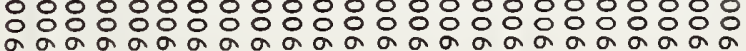





\section{THE NATIONAL BUREAU OF STANDARDS}

The scope of activities of the National Bureau of Standards at its major laboratories, in Washington, D.C., and Boulder, Colo., is suggested in the following listing of the divisions and sections engaged in technical work. In general, each section carries out specialized research, development, and engineering in the field indicated by its title. A brief description of the activities, and of the resultant publications, appears on the inside front cover.

WASHINGTON, D.C.

Electricity and Electronics. Resistance and Reactance. Electron Devices. Electrical Instruments. Magnetic Measurements. Dielectrics. Engineering Electronics. Electronic Instrumentation. Electrochemistry.

Optics and Metrology. Photometry and Colorimetry. Optical Instruments. Photographic Technology. Length. Engineering Metrology.

Heat. Temperature Physics. Thermodynamics. Cryogenic Physics. Rheology. Molecular Kinetics. Free Radicals Research.

Atomic and Radiation Physics. Spectroscopy. Radiometry. Mass Spectrometry. Solid State Physics. Electron Physics. Atomic Physics. Neutron Physics. Radiation Theory. Radioactivity. X-ray. High Energy Radiation. Nucleonic Instrumentation. Radiological Equipment.

Chemistry. Organic Coatings. Surface Chemistry. Organic Chemistry. Analytical Chemistry. Inorganic Chemistry. Electrodeposition. Molecular Structure and Properties of Gases. Physical Chemistry. Thermochemistry. Spectrochemistry. Pure Substances.

Mechanics. Sound. Mechanical Instruments. Fluid Mechanics. Engineering Mechanics. Mass and Scale. Capacity, Density, and Fluid Meters. Combustion Controls.

Organic and Fibrous Materials. Rubber. Textiles. Paper. Leather. Testing and Specifications. Polymer Structure. Plastics. Dental Research.

Metallurgy. Thermal Metallurgy. Chemical Metallurgy. Mechanical Metallurgy. Corrosion. Metal Physics.

Mineral Products. Engineering Ceramics. Glass. Refractories. Enameled Metals. Constitution and Microstructure.

Building Technology. Structural Engineering. Fire Protection. Air Conditioning. Heating and Refrigeration. Floor, Roof, and Wall Coverings. Codes and Safety Standards. Heat Transfer. Concreting Materials.

Applied Mathematics. Numerical Analysis. Computation. Statistical Engineering. Mathematical Physics.

Data Processing Systems. SEAC Engineering Group. Components and Techniques. Digital Circuitry. Digital Systems. Analog Systems. Applications Engineering.

- Office of Basic Instrumentation.

- Office of Weights and Measures.

\section{BOULDER, COLORADO}

Cryogenic Engineering. Cryogenic Equipment. Cryogenic Processes. Properties of Materials. Gas Liquefaction.

Radio Propagation Physics. Upper Atmosphere Research. Ionosphere Research. Regular Prediction Services. Sun-Earth Relationships. VHF Research. Radio Warning Services. Airglow and Aurora. Radio Astronomy and Arctic Propagation.

Radio Propagation Engineering. Data Reduction Instrumentation. Radio Noise. Tropospheric Measurements. Trophospheric Analysis. Propagation-Terrain Effects. Radio-Meteorology. Lower Atmosphere Physics.

Radio Standards. High-Frequency Electrical Standards. Radio Broadcast Service. Radio and Microwave Materials. Electronic Calibration Center. Microwave Physics. Microwave Circuit Standards.

Radio Communication and Systems. Low Frequency and Very Low Frequency Research.. High Frequency and Very High Frequency Research. Modulation Systems. Antenna Research. Navigation Systems. Systems Analysis Field Operations. 
